\title{
Cosmologia usando Aglomerados de Galáxias no Dark Energy Survey
}

\author{
Michel Aguena da Silva
}

Orientador: Prof. Dr. Marcos Vinicius Borges Teixeira Lima

Tese de doutorado apresentada ao Instituto de Física como requisito parcial para a obtenção do título de Doutor em Ciências

Banca Examinadora:

Prof. Dr. Marcos Vinicius Borges Teixeira Lima (IFUSP)

Prof. Dr. Luis Raul Weber Abramo (IFUSP)

Prof. Dr. Laerte Sodré Junior (IFUSP)

Prof. Dr. Gastão Cesar Bierrenbach Lima Neto (IAG)

Prof. Dr. Miguel Boavista Quartin (UFRJ)

São Paulo 


\section{FICHA CATALOGRÁFICA}

Preparada pelo Serviço de Biblioteca e Informação

do Instituto de Física da Universidade de São Paulo

Silva, Michel Aguena da

Cosmologia usando aglomerados de galáxias no dark energy survey / Cosmology with galaxy clusters in the dark energy survey — São Paulo, 2017.

Tese (Doutorado) - Universidade de São Paulo.Instituto de Física. Depto. de Física Matemática.

Orientador: Prof. Dr. Marcos Vinicius Borges Teixeira Lima Área de concentração: Cosmologia

Unitermos: 1. Física; 2. Cosmologia; 3. Aglomerados de galaxias; 4. Mecânica estatística; 5. Astronomia 


\section{Cosmology with Galaxy Clusters in the Dark Energy Survey}

\section{Michel Aguena da Silva}

Advisor: Prof. Dr. Marcos Vinicius Borges Teixeira Lima

Thesis submitted to the Physics Institute of the University of São Paulo in partial fulfillment of the requirements for the degree of Doctor in Science

\section{Thesis Committee:}

Prof. Dr. Marcos Vinicius Borges Teixeira Lima (IFUSP)

Prof. Dr. Luis Raul Weber Abramo (IFUSP)

Prof. Dr. Laerte Sodré Junior (IFUSP)

Prof. Dr. Gastão Cesar Bierrenbach Lima Neto (IAG)

Prof. Dr. Miguel Boavista Quartin (UFRJ)

São Paulo 

I dedicate this thesis to my mom and my Gabi, you inspire me every day. 



\section{Acknowledgement}

To my advisor Marcos Lima, for this opportunity into the overwhelming field of observational cosmology, for creating an environment where ideas could grow, and for straightforwards counsels about the professional path.

To mi hermano Hugo Camacho, my comrade in this wild endeavor of understanding the Universe. Without our many discussions and collaborations this work would not be possible.

To my family, specially my mother for always nourishing my yearn for learning and being there for me, and my aunt-mother for boosting my journey.

To my colleagues and friends, from our local cosmological group and the whole physics department, it would be an arduous road to walk alone. Specially to Vinicius Busti, who gave me a more pragmatic view of the path.

To the faculty from the physics and astronomy (IF and IAG) institutes at USP, for all my academic formation, and the faculty at Observatório Nacional and Observatoire de la Côte d'Azur, for fruitful collaboration and exposing an encouraging route for the future.

To CNPq (Conselho Nacional de Desenvolvimento Científico e Tecnológico, process $\mathrm{n}^{o}$ 142008/2012-5) and FAPESP (Fundação de Amparo à Pesquisa do Estado de São Paulo, process $\mathrm{n}^{\circ} 2013 / 26612-2$ ) for the fellowships that possibilitated my further pursuit into science and the realization of this work.

To Gabi, my timeless companion. 



\section{Abstract}

Galaxy clusters are the largest bound structures of the Universe. Their distribution maps the dark matter halos formed in the deep potential wells of the dark matter field. As a result, the abundance of galaxy clusters is highly sensitive to the expansion of the universe as well as the growth of dark matter perturbations, representing a powerful tool for cosmological purposes. In the current era of large scale surveys with enormous volumes of data, the statistical quantities from the objects surveyed (galaxies, clusters, supernovae, quasars, etc) can be used to extract cosmological information. The main goal of this thesis is to explore the potential use of galaxy clusters for constraining cosmology. To that end, we study the halo formation theory, the detection of halos and clusters, the statistical tools required to extract cosmological information from detected clusters and finally the effects of optical detection.

In the composition of the theoretical prediction for the halo number counts, we analyze how each cosmological parameter of interest affects the halo abundance, the importance of the use of the halo covariance, and the effectiveness of halos on cosmological constraints. The redshift range and the use of prior knowledge of parameters are also investigated in detail. The theoretical prediction is tested on a dark matter simulation, where the cosmology is known and a dark matter halo catalog is available. In the analysis of the simulation we find that it is possible to obtain good constraints for some parameters such as $\left(\Omega_{m}, w, \sigma_{8}, n_{s}\right)$ while other parameters $\left(h, \Omega_{b}\right)$ require external priors from different cosmological probes.

In the statistical methods, we discuss the concept of likelihood, priors and the posterior distribution. The Fisher Matrix formalism and its application on galaxy clusters is presented, and used for making forecasts of ongoing and future surveys. For the real analysis of data we introduce Monte Carlo Markov Chain (MCMC) methods, which do not assume Gaussianity of the parameters distribution, but have a much higher computational cost relative to the Fisher Matrix.

The observational effects are studied in detail. Using the Fisher Matrix approach, we care- 
fully explore the effects of completeness and purity. We find in which cases it is worth to include extra parameters in order to lower the mass threshold. An interesting finding is the fact that including completeness and purity parameters along with cosmological parameters does not degrade dark energy constraints if other observational effects are already being considered. The use of priors on nuisance parameters does not seem to affect the dark energy constraints, unless these priors are better than $1 \%$.

The WaZp cluster finder was run on a cosmological simulation, producing a cluster catalog. Comparing the detected galaxy clusters to the dark matter halos, the observational effects were investigated and measured. Using these measurements, we were able to include corrections for the prediction of cluster counts, resulting in a good agreement with the detected cluster abundance.

The results and tools developed in this thesis can provide a framework for the analyses of galaxy clusters for cosmological purposes. Several codes were created and tested along this work, among them are an efficient code to compute theoretical predictions of halo abundance and covariance, a code to estimate the abundance and covariance of galaxy clusters including multiple observational effects and a pipeline to match and compare halo/cluster catalogs. This pipeline has been integrated to the Science Portal of the Laboratório Interinstitucional de eAstronomia (LIneA) and is being used to automatically assess the quality of cluster catalogs produced by the Dark Energy Survey (DES) collaboration and will be used in other future surveys. 


\section{Resumo}

Aglomerados de galáxias são as maiores estruturas no Universo. Sua distribuição mapeia os halos de matéria escura formados nos potenciais profundos do campo de matéria escura. Consequentemente, a abundância de aglomerados é altamente sensível a expansão do Universo, assim como ao crescimento das perturbações de matéria escura, constituindo uma poderosa ferramenta para fins cosmológicos. Na era atual de grandes levantamentos observacionais que produzem uma quantidade gigantesca de dados, as propriedades estatísticas dos objetos observados (galáxias, aglomerados, supernovas, quasares, etc) podem ser usadas para extrair informações cosmológicas. Para isso, é necessária o estudo da formação de halos de matéria escura, da detecção dos halos e aglomerados, das ferramentas estatísticas usadas para o vínculos de parâmetros, e finalmente, dos efeitos da detecções ópticas.

No contexto da formulação da predição teórica da contagem de halos, foi analisada a influência de cada parâmetro cosmológico na abundância dos halos, a importância do uso da covariância dos halos, e a eficácia da utilização dos halos para vincular cosmologia. Também foi analisado em detalhes os intervalos de redshift e o uso de conhecimento prévio dos parâmetros (priors). A predição teórica foi testada um uma simulação de matéria escura, onde a cosmologia era conhecida e os halos de matéria escura já haviam sido detectados. Nessa análise, foi atestado que é possível obter bons vínculos cosmológicos para alguns parâmetros $\left(\Omega_{m}, w, \sigma_{8}, n_{s}\right)$, enquanto outros parâmetros $\left(h, \Omega_{b}\right)$ necessitavam de conhecimento prévio de outros testes cosmológicos.

Na seção dos métodos estatísticos, foram discutidos os conceitos de likelihood, priors e posterior distribution. O formalismo da Matriz de Fisher, bem como sua aplicação em aglomerados de galáxias, foi apresentado e usado para a realização de predições dos vínculos em levantamentos atuais e futuros. Para a análise de dados, foram apresentados métodos de Cadeias de Markov de Monte Carlo (MCMC), que diferentemente da Matriz de Fisher não assumem Gaussianidade entre os parâmetros vinculados, porém possuem um custo computacional muito mais alto. 
Os efeitos observacionais também foram estudados em detalhes. Usando uma abordagem com a Matriz de Fisher, os efeitos de completeza e pureza foram extensivamente explorados. Como resultado, foi determinado em quais casos é vantajoso incluir uma modelagem adicional para que o limite mínimo de massa possa ser diminuído. Um dos principais resultados foi o fato que a inclusão dos efeitos de completeza e pureza na modelagem não degradam os vínculos de energia escura, se alguns outros efeitos já estão sendo incluídos. Também foi verificados que o uso de priors nos parâmetros não cosmológicos só afetam os vínculos de energia escura se forem melhores que $1 \%$.

O cluster finder (código para detecção de aglomerados) WaZp foi usado na simulação, produzindo um catálogo de aglomerados. Comparando-se esse catálogo com os halos de matéria escura da simulação, foi possível investigar e medir os efeitos observacionais. A partir dessas medidas, pôde-se incluir correções para a predição da abundância de aglomerados, que resultou em boa concordância com os aglomerados detectados.

Os resultados a as ferramentas desenvolvidos ao longo desta tese podem fornecer um a estrutura para a análise de aglomerados com fins cosmológicos. Durante esse trabalho, diversos códigos foram desenvolvidos, dentre eles, estão um código eficiente para computar a predição teórica da abundância e covariância de halos de matéria escura, um código para estimar a abundância e covariância dos aglomerados de galáxias incluindo os efeitos observacionais, e um código para comparar diferentes catálogos de halos e aglomerados. Esse último foi integrado ao portal científico do Laboratório Interinstitucional de e-Astronomia (LIneA) e está sendo usado para avaliar a qualidade de catálogos de aglomerados produzidos pela colaboração do Dark Energy Survey (DES), assim como também será usado em levantamentos futuros. 


\section{Contents}

1 Introduction 1

1.1 Cosmological Surveys and Challenges . . . . . . . . . . . . . . . 1

1.2 Basics of Cosmology $\ldots \ldots \ldots \ldots \ldots$

1.2 .1 Metric . . . . . . . . . . . . . . . . 4

1.2 .2 Hubble Parameter . . . . . . . . . . . . . . . . . . . 6

1.2 .3 Friedmann Equations $\ldots \ldots \ldots \ldots \ldots$

1.2 .4 Comoving Radial Distance . . . . . . . . . . . . . . . 8

1.2.5 Comoving Angular Diameter Distance . . . . . . . . . . . . . . . . . 8

1.2 .6 Comoving Volume $\ldots \ldots \ldots \ldots$. . . . . . . . . . . . . 9

1.2.7 $\Lambda$ CDM - The Standard Cosmological Model $\ldots . . . . . . . . . .99$

$1.2 .8 \quad$ Power Spectrum and Density Variance . . . . . . . . . . . . . . . . . 11

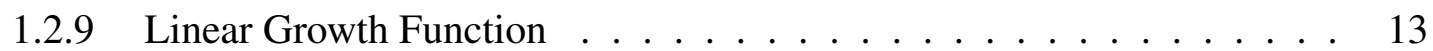

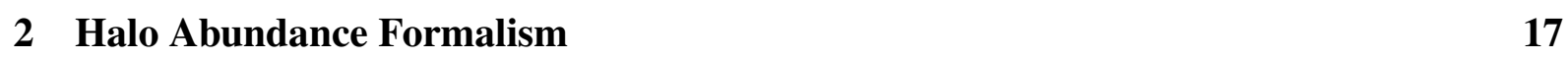

2.1 Spherical Collapse $\ldots \ldots \ldots \ldots$. . . . . . . . . . . . . . 17

2.2 Filtering and Window Function . . . . . . . . . . . . . . . 21

2.3 Halo Mass-function . . . . . . . . . . . . . . . . . . . 22

2.3 .1 Press-Schechter Mass-function . . . . . . . . . . . . . . . . . . . 24

2.3 .2 Sheth \& Tormen Mass-function . . . . . . . . . . . . . . . . . . 24

2.3 .3 Jenkins Mass-function $\ldots \ldots \ldots \ldots$

2.3 .4 Tinker Mass-function . . . . . . . . . . . . . . . . . . . . 25

2.4 Halo Number Counts . . . . . . . . . . . . . . . . . . . . . . . . 26

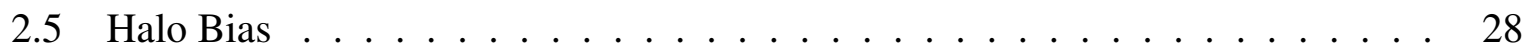

2.5 .1 Press-Schechter Bias . . . . . . . . . . . . . . . . . . . . 29

2.5 .2 Sheth-Tormen Bias . . . . . . . . . . . . . . . . . 30 
2.5 .3 Tinker Bias . . . . . . . . . . . . . . . . . . . . . 30

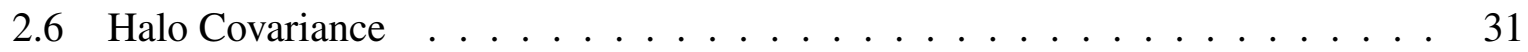

2.6 .1 Full sky volume. . . . . . . . . . . . . . . . . . . . . 32

2.6 .2 Full sky shell $\ldots \ldots \ldots \ldots \ldots \ldots \ldots \ldots$

2.6 .3 Cylindrical window $\ldots \ldots \ldots \ldots \ldots \ldots \ldots \ldots$

2.6 .4 Cubical Window . . . . . . . . . . . . . . . . . . 34

2.6 .5 Irregular windows $\ldots \ldots \ldots \ldots \ldots \ldots \ldots \ldots$

$2.6 .6 \quad$ Poisson Variance and Total Covariance $\ldots \ldots \ldots \ldots$. . . . . . 35

\begin{tabular}{|ll|}
3 & Observations of Galaxy Clusters \\
\hline
\end{tabular}

3.1 Techniques for Detecting Clusters $\ldots \ldots \ldots \ldots \ldots$

$3.1 .1 \quad$ Optically Detected Clusters $\ldots \ldots \ldots \ldots$

3.1 .2 Other Methods for Cluster Detection. . . . . . . . . . . . . . . . . . 39

$3.2 \quad$ Halo and Cluster Finders $\ldots \ldots \ldots \ldots$. . . . . . . . . . . . . . 41

$3.2 .1 \quad$ Optical Cluster Finders: redMaPPer, VT and WaZp $\ldots \ldots \ldots \ldots$

3.3 Observational Effects of Galaxy Clusters . . . . . . . . . . . . . . . 45

3.3 .1 Photometric Redshifts . . . . . . . . . . . . . . . . . . . 46

3.3 .2 Mass-Observable Relation . . . . . . . . . . . . . . . . . 47

3.3 .3 Completeness and Purity . . . . . . . . . . . . . 50

3.3.4 Theoretical Prediction for the Abundance of Observed Clusters _. . . 51

4 Statistical Methods $\quad 53$

4.1 Constraining Parameters $\ldots \ldots \ldots \ldots \ldots \ldots$

4.1 .1 Likelihood . . . . . . . . . . . . . . . . . . . . 54

4.1 .2 Marginalizing versus Fixing parameters $\ldots \ldots \ldots \ldots \ldots$

4.2 Fisher Matrix $\ldots \ldots \ldots \ldots \ldots \ldots \ldots \ldots$

$4.2 .1 \quad$ Gaussian Likelihood . . . . . . . . . . . . . . . . . . . . . . . . 59

4.2 .2 Poisson Likelihood . . . . . . . . . . . . . . . . . . . . . 60

4.2 .3 Interpolated Likelihood $\ldots \ldots \ldots \ldots$. . . . . . . . . . . . . 61

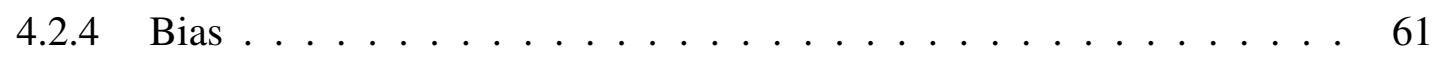

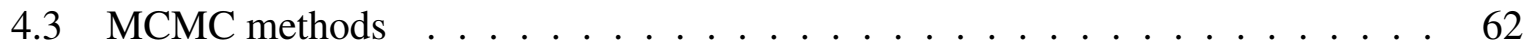

4.4 Estimation of Sample Covariance from Observations . . . . . . . . . . . . . 64

4.4 .1 Sub-sampling . . . . . . . . . . . . . . . . . . . . . 64 
4.4 .2 Jackknife Sub-sampling . . . . . . . . . . . . . . . . . 65

4.4 .3 Bootstrap Sub-sampling . . . . . . . . . . . . . . . 65

4.4 .4 Multiple Simulation Sampling . . . . . . . . . . . . . . . 66

\begin{tabular}{|lll}
5 & Theoretical Results & 67
\end{tabular}

5.1 Mass-Observable relation . . . . . . . . . . . . . . . . . . . . 67

5.2 Cosmological Parameters from Dark Matter Halos . . . . . . . . . . . . 73

5.3 Optical Effects on Cluster Dark Energy Constraints . . . . . . . . . . . . . . 80

5.3 .1 Fiducial Model . . . . . . . . . . . . . . . . . . 81

5.3 .2 Cluster Number Counts $\ldots \ldots \ldots$

5.3 .3 Selecting Cases $\ldots \ldots \ldots \ldots$. . . . . . . . . . . . . 85

5.3 .4 Completeness and Purity Effects . . . . . . . . . . . . . . . 88

$5.3 .5 \quad$ Future Surveys $\ldots \ldots \ldots$. . . . . . . . . . . . . . . 92

6 Simulation Results 95

$6.1 \quad$ MICE SIMULATION $\ldots \ldots \ldots \ldots$

6.2 DES Aardvark v1.0 SIMULATION . . . . . . . . . . . . . . . . . . . . . 99

$6.2 .1 \quad$ Measurement of Halo Abundance and Sample Variance. . . . . . . . . 101

6.2 .2 Cosmology from Dark Matter Halos . . . . . . . . . . . . . . . 104

6.2 .3 WaZp Clusters . . . . . . . . . . . . . . . . . . 106

\begin{tabular}{llr}
\hline 7 & Conclusions & 125
\end{tabular}

\begin{tabular}{ll}
\hline Appendices & 129
\end{tabular}

\begin{tabular}{|lll}
\hline A & Estimating Galaxy Cluster Properties in Simulations & 131
\end{tabular}

A.1 Matching of Halos and Clusters f . . . . . . . . . . . . . . . 131

A.2 Measuring Completeness and Purity . . . . . . . . . . . . . . 133

A.3 Estimating the Mass-proxy relation . . . . . . . . . . . . . . . . . . 134 


\section{List of Figures}

1.1 Evolution of scales of the Universe for different values of curvature $k$ in units

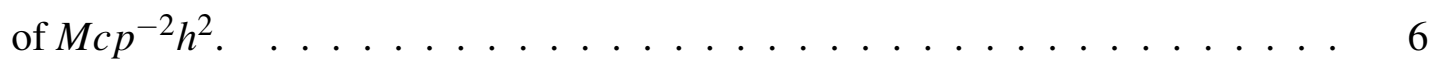

1.2 Hubble factor evolution with redshift using the Planck cosmology (Table 1.1). The solid line is the Hubble factor with all components, and the dashed lines are the contribution of each component. . . . . . . . . . . . . 11

1.3 Matter power spectrum with Planck Collaboration et al.(2016) cosmology. . . 13

1.4 Linear growth function versus redshift for different cosmological models. Left: flat $\Lambda$ CDM model. Right: flat $w$ CDM model with $\Omega_{m}=0.25$. . . . . . . . . 15

2.1 Differential comoving mean halo number density as a function of halo mass at $z=0$, for the different mass functions in a $\Lambda$ CDM model. The lower panel shows the ratios of the different mass functions relative to that of Press \& Schechter. . . . . . . . . . . . . . . . . . . 26

2.2 Halo number counts for $M>10^{13.8} M_{\odot} h^{-1}$ with fiducial cosmology defined in section 2.4 and a positive variation of $20 \%$ in each cosmological parameter keeping all the others fixed. On the bottom panel displays the relative variation relative to the fiducial case. The increase of $\Omega_{m}, \sigma_{8}, n_{s}$ and $h$ result in an increase of the abundance of halos, while an increment of $\Omega_{b}$ leads to a decrease on the halo abundance. The variation with $w$ is not monotonic, leading to a smaller abundance of halos at lower redshifts $(z \lesssim 1.1)$ and an increase at higher

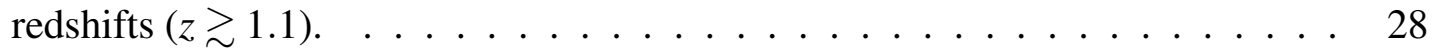

2.3 Halo bias as a function of halo mass at $z=0$, for the different mass functions in a $\Lambda$ CDM model. The lower panel shows the ratios of the different mass functions relative to that of Press \& Schechter. . . . . . . . . . . . . . . . 31 
2.4 Diagonal of the cluster covariance, with the Tinker mass function in a cylindrical window of $10 \mathrm{deg}^{2}$ for different minimum mass thresholds. The total covariance (black solid line) is show along with the individual contributions from Poisson noise (blue dotted line) and Sample covariance (red dashed line). 36

3.1 Snapshot of the procedures in the WaZp code. Left: An overdensity across redshift slices. Right: Membership assignment schematics.

4.1 Example of how fixing one parameter at a certain $\left(\Theta_{2}=-3:\right.$ red solid line) can lead to a erroneous best fit for the other parameters $\left(\Theta_{1}=\max \left(\mathcal{L} \mid \Theta_{2}=-3\right)=\right.$ $2.4:$ red dashed line). . . . . . . . . . . . . . . . . 56

4.2 Example of how the best fit of a marginalized parameter (blue solid lines) can differ from the best fit overall (red dashed lines). . . . . . . . . . . . . . . . . . 57

$5.1 \quad$ Probability density space $d V_{p}\left(M, M^{\text {obs }}\right)$ (Eq. $5.1 p$ for a Gaussian $P\left(M^{\text {obs }} \mid M\right)$ with fixed scatter and no bias. The black line is the diagonal, the gray dashed line is defined by $l_{M_{\max }}$ (Eq. 5.5$)$ and the gray dotted line is given by $l_{M_{\max }^{\text {obs }}}$ (it coincides completely with the diagonal). . . . . . . . . . . . . 70

$5.2 \quad$ Mass scatter $\sigma_{\ln M}$ and mass bias $\ln M^{\text {bias }}$ of $P\left(M \mid M^{\text {obs }}\right)$ resulting from a Gaus$\operatorname{sian} P\left(M^{\text {obs }} \mid M\right)$ with fixed scatter and no bias. $\ldots \ldots \ldots . \ldots 71$

5.3 Gaussian distribution $P\left(M^{\mathrm{obs}} \mid M\right)$ (black dashed line) with fixed scatter and no bias, the resulting $P\left(M \mid M^{\text {obs }}\right)$ (blue solid line), and Gaussian distribution (red dotted line) with scatter $\sigma_{\ln M}$ and mass bias $\ln M^{\text {bias }}$ fitted in Fig. 5.2 for several

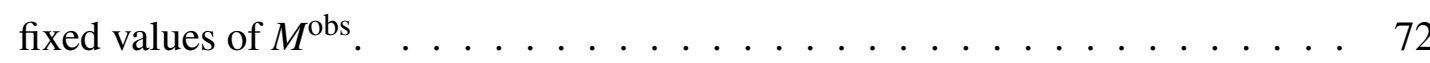

5.4 Difference between the measured moments $p_{k}$ of $P\left(M \mid M^{\text {obs }}\right)$ and the Gaussian moments $G p_{k}$ computed from the first two moments $p_{1}$ and $p_{2} . \ldots$. . . . . . 73

5.5 Constraints on cosmological parameters using the abundance of halos only for different redshift ranges. The blue solid area are using all halos, in green only the lower redshift halos $(z<1.0)$ and in red only the higher redshift halos $(z>1.0)$. For each panel only the parameters shown were varied, fixing all other parameters. . . . . . . . . . . . . . . . . . 75

5.6 Constraints on cosmological parameters using the abundance of halos without any prior knowledge. . . . . . . . . . . . . . . . . . 77 
5.7 Constraints of cosmological using the abundance of halos with Gaussian priors

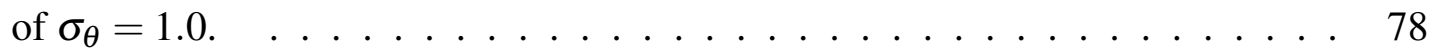

5.8 Constraints on cosmological parameters using both the abundance and covariance of halos without priors. . . . . . . . . . . . . . . . . . 79

5.9 Completeness and Purity behavior for cases (1) and (2) at $z=0$. The red vertical line is the threshold mass $M_{t h}^{\text {obs }}=10^{13.8} M_{\odot} / h$ assumed in the fiducial model. Left: Both functions are displayed separated and the red shaded regions (on the top figure) display the mass spread around this threshold at 1,2 and 3 times \begin{tabular}{lll}
$\sigma_{\ln M}$ obs. & Right: Ratio of completeness over purity as it is used in Eqs. & 3.13 \\
\hline
\end{tabular} and 3.14, Case (1) will result in a increment on the abundance of clusters for higher masses and a deficiency at lower masses, while case (2) has the opposite behavior. . . . . . . . . . . . . . . . . . . 83

5.10 Variation of the number counts of the case (1) with respect to the cosmological and nuisance parameters. The black solid line is the fiducial case, the colored lines are a positive variation of 0.2 for each parameter. . . . . . . . . . . . 84

5.11 Bias $b\left(\Theta_{\alpha}\right)$ on dark energy parameters caused by ignoring completeness and purity effects on different cases. The blue shaded areas are the $68 \%, 95 \%$ and 99\% confidence levels assuming perfect cluster detection. The solid, and dashed lines correspond to the cases (1) and (2) respectively (see text). When the bias becomes larger than the $1 \sigma$ constraints $b\left(\Theta_{\alpha}\right)>\sqrt{F_{\alpha \alpha}}$, the assumption of perfect detection results in systematically incorrect cosmological predictions. For the threshold mass considered of $\log \left[M_{t h}^{\text {obs }} /\left(M_{\odot} h^{-1}\right)\right]=13.8$, we have that the bias of $\Omega_{\mathrm{DE}}$ is much larger than $\sigma$ relative to both cases $(1,2)$ and the bias on $w$ is of the same order of $\sigma . \ldots \ldots \ldots \ldots$

5.12 Constraints on dark energy parameters for different cases. The shaded area are the constraints of case $(0)$ with the threshold $\log \left[M^{\text {obs }} /\left(M_{\odot} h^{-1}\right)\right]=14.2$,

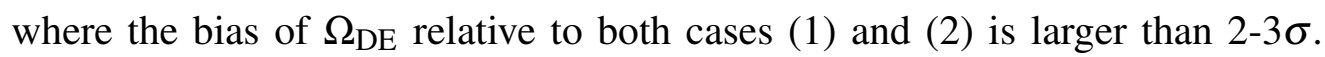
Both cases considering completeness and purity with a lower mass threshold $\log \left[M^{\text {obs }} /\left(M_{\odot} h^{-1}\right)\right]=14.2$ actually have better constraints than ignoring these effects and restraining to higher mass thresholds. . . . . . . . . . . . . . 87 
5.13 Constraints from the Fisher Matrix approach on observational effects. In the left are the constraints derived for nuisance parameters in case (1) (blue solid line) and case (2) (green dashed line). In the right are the constraints for $\Omega_{\mathrm{DE}}$ and $w$ resulting by adding individual prior in each nuisance parameter. The parameters are related to the observable-mass relation (top panel) and completeness/purity (bottom panel). No prior knowledge was assumed for these nuisance parameters. . . . . . . . . . . . . . . . . . . . . 90

5.14 Degradation on dark energy constraints for case (1) with priors on the nuisance paremeters relative to the case where these parameters are fixed (Eq. 5.13). For a given value of $\sigma_{\mathrm{p}}\left(\Theta_{O M}\right)$, the same prior is applied on all the richness-mass relation parameters. The same happens for $\sigma_{\mathrm{p}}\left(\Theta_{C P}\right)$ and the completeness/purity parameters. . . . . . . . . . . . . . . . . . . 92

5.15 Dark energy constraints considering different values of maximum redshift $z_{\max }$.

5.16 Effects of completeness and purity as a function of maximum redshift $z_{\max }$. On \begin{tabular}{|c|}
\hline the left is shown the degradation of dark energy constraints for the case where \\
\hline
\end{tabular} completeness and purity parameters are free $\left(\sigma_{p}\left(\Theta_{C P}\right)=\infty\right)$ relative to the case \begin{tabular}{|c|}
\hline where these parameters are fixed $\left(\sigma_{p}\left(\Theta_{C P}\right)=0\right)$. On the right are shown the \\
\hline
\end{tabular} actual dark energy constraints in both cases. . . . . . . . . . . . . . . . 94

6.1 Number counts in the MICE simulation for halos with redshifts $0.5<z<0.6$.

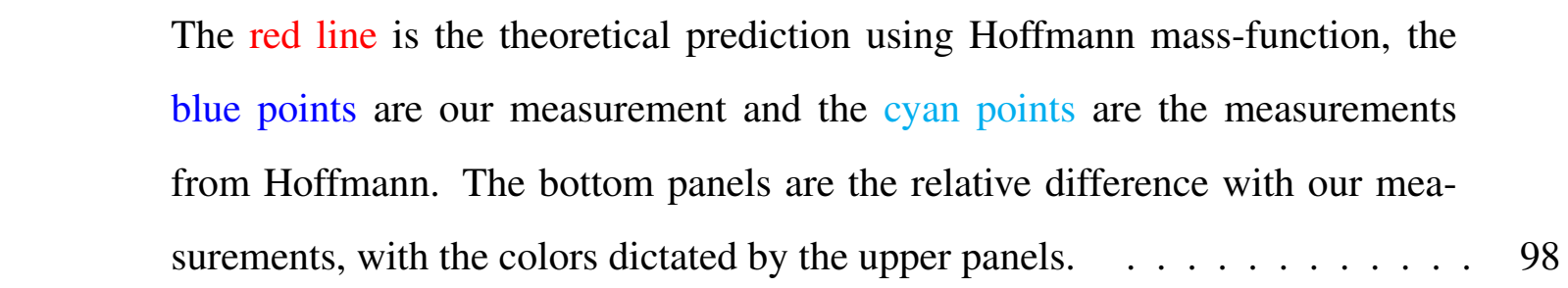

6.2 Total variance in the MICE simulation for halos with redshifts $0.5<z<0.6$.

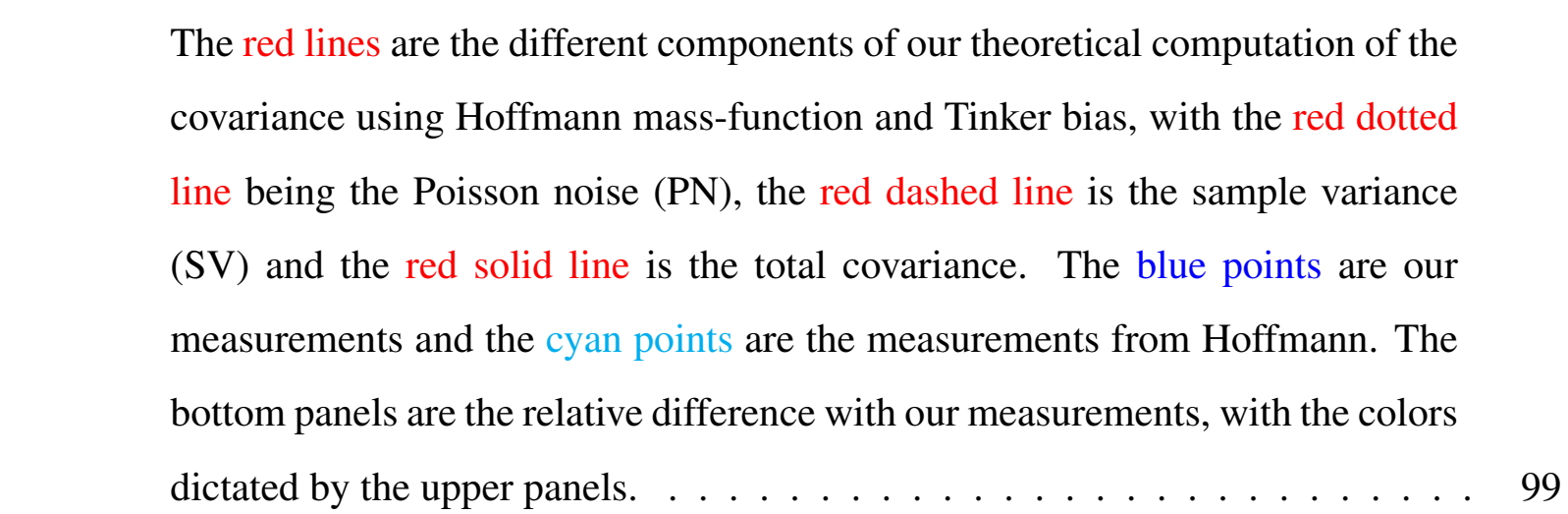


6.3 Total variance measured in the MICE simulation with different methods and sub-divisions, along with theoretical predictions. The theoretical predictions is displayed in gray lines with the style changing with the area. The measurement methods are separated by line styles as: Sub-sampling (SS); Sub-sampling rescaled (SSr); Jack-Knife (JK); Bootstrap (BS). The colors distinguish the area splitting method: (N4) blue; (N16) red; (N4S) green . . . . . . . . . . . . . 100

6.4 Number counts measured in the Aardvark v1.0 simulation along with the theoretical prediction with different areas and mass bins. The blue points and error-bars are the measurements for the average counts and their total variance, respectively, whereas the red lines and shaded regions are the theoretical predictions for the counts and total covariance, respectively. The mass bins are \begin{tabular}{|c|c|c|}
\hline displayed on the top right corner of each sub-panel in units of $\log \left(M / M_{\odot} h^{-1}\right)$. & 102
\end{tabular}

6.5 Total variance measured in the DES Aardvark v1.0 simulation using the multiple samples created. . . . . . . . . . . . . . . . . . 103

6.6 Relative difference between total covariance measured in the DES Aardvark \begin{tabular}{|c|}
\hline v1.0 simulation and theory predictions including Poisson variance and sample \\
\hline
\end{tabular} covariance. . . . . . . . . . . . . . . . . . . . . . . . . 104

6.7 Cosmological constraints with dark matter halos in the BCC simulation. The parameters $\left(h, n_{s}, \Omega_{b}\right)$ were fixed at their fiducial values. The filled regions are the forecasts using the Fisher Matrix and the solid lines are the results from the MCMC on the catalog measurements. . . . . . . . . . . . . 106

6.8 Cosmological constraints with dark matter halos in the BCC simulation. The filled regions are the forecasts using the Fisher Matrix and the solid lines are the results from the catalog measurements. . . . . . . . . . . . . 107

6.9 Halo number counts (red points) measured in the BCC simulation and theoretical predictions at the the best fit parameters found in the MCMC chain (lines). The blue solid line is the result of allowing all cosmological parameters to vary \begin{tabular}{|l}
\hline in the chain, and the green dashed line is for the case where only $\left(\Omega_{m}, w, \sigma_{8}\right)$ \\
\hline vary. $\ldots \ldots \ldots \ldots \ldots \ldots \ldots \ldots \ldots \ldots \ldots \ldots \ldots \ldots \ldots \ldots \ldots \ldots \ldots \ldots \ldots \ldots$
\end{tabular}

6.10 Angular density of matter halos (left) and WaZp clusters (right) of the Aardvark v1.0 catalog of the BCC simulation. . . . . . . . . . . . . . . 109 
6.11 Distribution of dark matter halos (left) and WaZp clusters (right) in the Aardvark v1.0 catalog of the BCC simulation as a function of mass/richness and redshift. 110

6.12 Number of dark matter halos and WaZp clusters matched in the BCC simulation with different matching parameters. In the left are the number of halos (top) and clusters (bottom) matched considering multiple matches and in the right is the number of matched halos and clusters with cross match only. . . . . . . . . 111

6.13 Distribution of the matched pairs as a function of normalized redshift distance, binned by mass (top left), richness (top right), halo redshift (bottom left) and cluster redshift (bottom right). . . . . . . . . . . . . . . . . . 112

6.14 Distribution of the matched pairs as a function of angular distance converted to physical distance and normalized by the halo radius $R_{200}$, binned by mass (top left), richness (top right), halo redshift (bottom left) and cluster redshift (bottom right) $\ldots \ldots \ldots \ldots \ldots \ldots \ldots \ldots$

6.15 Completeness (left) and purity (right) of the WaZp catalog in the BCC simulation by pixels in the footprint. . . . . . . . . . . . . . . . . . . . 114

6.16 Completeness (left) and purity (right) of the WaZp catalog in the BCC simulation by mass/richness and redshift. . . . . . . . . . . . . . . . . . 115

6.17 Completeness and purity as a function of mass/richness in several redshift bins. The points are the measurements and the lines are a fit according to Eqs. 3.13 and $3.14 \square \ldots \ldots \ldots \ldots \ldots \ldots$

6.18 Relation between the halo and cluster redshifts in the BCC simulation. . . . . . 116

6.19 Mass Richness diagram for the matched halos with WaZp clusters in the BCC simulation and the fitted parameters. Left: The red dots are the matched pairs, the green points are the data binned with the measured scatter, the yellow points are the bins not used for the mass-richness fitting, the cyan dashed line is a fit for the mass-richness relation in each bin and the blue line is the redshift fit of the mass-richness parameters. Right: Parameters fitted in each redshift bin (points) and the redshift fit of the parameters (line). On Top all redshift bins were used for the fit and on the bottom the first bin was excluded, resulting in a better fit for the overall data. . . . . . . . . . . . . . . . . . . . . . . . 119 
6.20 Measured scatter for each different binning. In the left the data was binned in mass and in the right in richness. The mass of the clusters (right plot) was computed from the fit. Each panel is the data in a redshift bin labeled at the bottom left. . . . . . . . . . . . . . . . . . . . . . . . 120

6.21 Number counts of WaZp clusters detected (blue steps) in a reduced region $(\approx 220$ $\mathrm{deg}^{2}$ ) of the BCC simulation and the corresponding theoretical prediction (red line) for three richness thresholds $N_{t h}=20,60,100$ (top to bottom). The shaded regions are the $1,2,3 \sigma_{\bar{m}}$ computed with Eq. 6.7$\rceil \ldots \ldots$. . . . . . . . . . 121

6.22 Number counts of WaZp clusters detected (blue filled regions) in the BCC simu-

\begin{tabular}{|l|}
\hline lation and theoretical prediction for three richness thresholds. Four approaches \\
\hline were considered: Perfect detection and no scatter on the mass-richness relation \\
\hline (thin black line); Including the completeness and purity, but no scatter (blue \\
\hline
\end{tabular}
dashed line); Perfect detection with scatter (blue dotted line); Considering all



6.23 Ratio of completeness and purity for different masses/richnesses at $z=0.55$.

\begin{tabular}{|l|}
\hline Left: The black line corresponds to a mass computed form the richness using \\
\hline the mass richness relation, in the green line the mass was computed from a \\
\hline positive variation of $\sigma_{\ln N_{\text {gal }}}$ on the richness on the richness and on the red line \\
\hline with a negative. Right: The color map was arranged for red corresponding to \\
\hline$c<p$, white to $c=p$ and the other colors to $c>p$. The solid line is for the mass \\
\hline richness relation calibrated, and the dashed lines are for 1,2 and $3 \sigma$ variations. \\
\hline
\end{tabular}




\section{Chapter 1}

\section{Introduction}

\subsection{Cosmological Surveys and Challenges}

The study of cosmology has experienced a rapid progress in the last few decades. From a field of mostly theoretical models and a few concrete measurements, to a science remarkably rich in both observational data and mathematical models, the approach on cosmological research underwent a significant change. Currently, it is possible to make predictions and measurements not only for the background Universe, such as the accelerated expansion, but also for the perturbations around this background, such as the abundance of massive objects and correlations describing large scale structures. Within General Relativity, the energy content of the Universe at the present epoch has been well characterized as dark energy $(\approx 70 \%)$, dark matter $(\approx 25 \%)$ and the baryonic matter $(\approx 5 \%)$, a picture which has been possible to draw thanks to surveys such as WMAP ${ }^{1}$. Hinshaw et al., 2013) and Planck 2 (Planck Collaboration et al., 2016), which investigated the fluctuations in the cosmic microwave background (CMB) radiation. However, the nature of the predominant elements (dark energy and dark matter) is yet unknown. The understanding of these components could lead to significant implications on several fields of Physics such as gravitation, particle physics, quantum field theory and cosmology itself.

In addition to past and current $\mathrm{CMB}$ experiments, optical surveys of galaxies provide us with another set of probes to investigate cosmological models at much lower redshifts. These probes include supernovae, baryon acoustic oscillations (BAO), gravitational lensing and the abundance of galaxy clusters. Using supernovae (Perlmutter et al., 1997; Riess et al., 1998;

\footnotetext{
1 http://map.gsfc.nasa.gov

2 http://www.cosmos.esa.int/web/planck
} 
Kowalski et al., 2008), quasars (Carroll et al., 1992; York et al., 2000) and the background temperature of the $\mathrm{CMB}$, it is possible to probe the background universe, i.e. scales large enough in which the universe can be considered homogeneous and isotropic. The fluctuations of the $\mathrm{CMB}$, correlations of matter tracers, gravitational lensing and the abundance of galaxy clusters (Komatsu et al., 2011; Planck Collaboration et al., 2016) contain information on the fluctuations around the homogeneous Universe, which drive structure formation.

Galaxy clusters are the largest structures in the Universe. Their abundance depends exponentially on density fluctuations in the Universe, and is extremely sensitive to parameters describing cosmic expansion as well as the growth of perturbations. As a result, cluster abundance can be used to constrain modified gravity theories (Tang et al., 2006), the mass of neutrinos (Wang et al., 2005), the total matter density (Gladders et al., 2007), the amplitude of the primordial fluctuations (Rozo et al., 2007) and help distinguish between competing dark energy models, such as a cosmological constant and a dynamical dark energy (Eke et al., 1996; Holder et al. 2001; Evrard et al., 2002). However, in order to use clusters for cosmological purposes, several practical problems must be considered and properly accounted for. One of the main issues in this context, which affects clusters detected on all wavelengths, is the estimation of the masses of galaxy clusters. Except for cluster lensing measurements, cluster masses cannot be accurately inferred from astronomic measurements alone, and must be inferred through some observable, via a observable-mass relation and its scatter (Lima \& Hu, 2004, 2005). The expected number of clusters depends significantly on the threshold mass above which we count them. Additionally, one must also quantify the frequency of cluster mis-detections due to intrinsic problems in cluster finder algorithms as well as observational issues such as blending of objects due to projection effects.

The modern approach on constraining cosmology relies on the use of large surveys where statistical quantities can be rigorously measured and compared to theoretical predictions and simulations. Current and future surveys include the Sloan Digital Sky Survey ${ }^{3}$ (SDSS)(York et al. 2000), the Canada-France-Hawaii Telescope Legacy Survey ${ }^{4}$ CFHTLS)(Pierre et al., 2004), the Dark Energy Survey 5 (DES) (Flaugher, 2005), the Javalambre Physics of the Accelerating Universe Survey 6 (JPAS) Cenarro et al., 2012), the Large Synoptic Survey Telescope

\footnotetext{
$\sqrt[3]{\text { http: //www.sdss.org }}$

${ }^{4}$ http://www.cfht.hawaii.edu/Science/CFHTLS

5 http://www.darkenergysurvey.org

6 http://j-pas.org
} 
7 (LSST)(Tyson, 2002) and Euclid 8 (Amendola et al., 2013). The aim of the present work is to focus on the power of cluster cosmology specifically within the DES. However, the same techniques can be applied to different optical surveys and even for clusters detected in other wavelengths such as X-ray and millimeter. Towards that goal, it is critical to construct an endto-end analysis for the cosmological use of galaxy clusters. This analysis can then be validated on cosmological simulations before being applied to real observations. In this process we learn about the best approaches and most efficient parametrizations to use, which otherwise would be difficult to assess.

The DES (Flaugher, 2005) is a wide-field imaging program, designed to probe the background expansion and growth of structure in the Universe, thereby helping uncover the nature of dark energy via the self-consistent combination of the cosmological probes mentioned above. Over 400 scientists from 23 institutions in the United States, the United Kingdom, Spain, Germany, Switzerland, Australia and Brazil have been working on the project. Starting in September 2013 and continuing for five years, DES will survey $\approx 5000 \mathrm{deg}^{2}$ of the southern sky in the griz $Y$ bands down to magnitudes $\approx 24.5$ out to vast distances in order to provide constraints on models of cosmic acceleration. For these observations, a new CCD optical camera DECam (Dark Energy Camera) (Flaugher et al., 2015) was developed by the DES collaboration and installed at the Blanco 4-meter telescope, at the Cerro Tololo Inter-American Observatory (CTIO) in Chile. The DECam images are reduced and calibrated by the DES Data Management (DESDM) team at the National Center for Supercomputing Applications (NCSA) which has developed a pipeline to process the data from basic single exposures, taking into account instrumental corrections all the way to the creation of a source catalog from calibrated coadded images as described by (Mohr et al. 2012). During the observation over 525 nights, it is expected to record information on over 300 million galaxies from redshifts $\approx 0.2$ up to $\approx 2$.

While the real data is being collected, several mock catalogs have been made for preliminary tests. For our purposes, these tests include running different galaxy cluster finders and applying cosmological parameter estimators. Some of the results presented here are based on the Blind Cosmology Challenge (BCC) mock catalog produced by the DES collaboration, which consists of $\mathrm{a} \sim 10.000 \mathrm{deg}^{2}$ area catalog of halos and galaxies up to redshift $z \approx 2.0$.

In the next section we will briefly describe the basic formalism of the standard cosmological framework as well as the main tools necessary for the study of cluster cosmology. Chapter

\footnotetext{
7 https://www.lsst.org/

8 http://www.euclid-ec.org
} 
2 lays the formalism developed concerning the abundance of dark matter halos. From basic principles to improvements using simulations, we introduce the theoretical foundation for an accurate prediction of halos. In Chapter 3 , we discuss the observational challenges of detecting galaxy clusters and their intrinsic connection to the dark matter halos. The possible techniques to probe galaxy clusters and the computational algorithms will be described, with special focus on detection on the optical spectrum. Additionally, we discuss the observational effects and how to incorporate them on the theoretical prediction. The statistical concepts and tools to extract the cosmological information from large amount of data concerning galaxy clusters will be presented in Chapter 4 . Chapter 5 contains the results from theoretical results only, where the mass-proxy and Fisher Matrix results will be explored. In Chapter 6, we analyze two distinct simulations from MICE and DES collaborations. Finally, in Chapter7, we present the summary, conclusions and prospects of this thesis.

\subsection{Basics of Cosmology}

The main equations describing the evolution of cosmological quantities are derived from General Relativity (GR), the best gravitational theory known to date. In this section, the basis for the cosmological equations will be briefly presented and some important concepts of cosmology to work with galaxy clusters will be introduced.

The interaction between matter and the space-time is described by the Einstein equations

$$
G_{\mu v}-\Lambda g_{\mu v}=8 \pi G T_{\mu \nu}
$$

with $G_{\mu \nu}$ being the Einstein tensor, $\Lambda$ the cosmological constant and $T_{\mu \nu}$ the energy momentum tensor. Given a certain metric $g_{\mu \nu}$ and the energy-momentum components (i. e. matter,radiation,...), Eq.1.1 determines the dynamics of the Universe.

\subsubsection{Metric}

According to the cosmological principle, there is no privileged position or direction in the largescale universe. Therefore the Universe must be homogeneous and isotropic. The FriedmannRobertson-Walker (FRW) metric describes a Universe with such conditions and also includes 
the possibility of a spatial curvature. The FRW metric with curvature $k$ is given by

$$
d s^{2}=d t^{2}-a^{2}(t)\left[d \chi^{2}+f^{2}(\chi) d \alpha^{2}\right]
$$

where $\chi$ is the comoving radial distance, $a(t)$ is the scale factor, $d \alpha^{2}=d \theta^{2}+\sin ^{2} \theta d \phi^{2}$ and

$$
f(\chi)=\left\{\begin{array}{cc}
(\sqrt{k})^{-1} \sin (\chi \sqrt{k}) & ; k>0 \\
\chi & ; k=0 \\
(\sqrt{-k})^{-1} \sinh (\chi \sqrt{-k}) & ; k<0
\end{array}\right.
$$

The scale factor $a(t)$ scales the comoving spatial part of the metric due to the expansion of the Universe. In this description, the comoving distance multiplied by the scale factor results in the physical distance. Therefore, two objects with no proper motion have a fixed comoving distance, yet, because of the expansion (or in some cosmological models, contraction) of the Universe, their physical distance can change. Generally, the scale factor is normalized by its value today $\left(t_{0}\right)$, resulting in $a_{0}=a\left(t_{0}\right) \equiv 1$.

Depending on the value of $k$, the Universe can be considered flat $(k=0)$, open $(k<0)$ or closed $(k>0)$. In Fig. 1.1 is the evolution of $f(\chi)$ for several values of curvature are shown. For the closed solutions, there is always a maximum value $f(\chi)$ can reach, reducing afterwards to zero. The solution of the flat and open Universes diverge only for $\chi \rightarrow \infty$, with $k<0$ growing faster.

We can compute the redshift due to cosmological expansion, by considering a photon detected today $\left(t_{0}\right)$ that was emitted at a instant $t_{e}$ as

$$
z=\frac{\lambda_{0}-\lambda_{e}}{\lambda_{e}}=\frac{a\left(t_{0}\right) \lambda_{c}-a\left(t_{e}\right) \lambda_{c}}{a\left(t_{e}\right) \lambda_{c}}=\frac{1-a\left(t_{e}\right)}{a\left(t_{e}\right)} .
$$

where $\lambda_{0}$ is the wavelength of the photon measured today, $\lambda_{e}$ the wavelength of the photon at the source and $\lambda_{c}$ would correspond to a comoving wavelength. From this result, we have that the scale factor at a time $t$ where the redshift is $z$ is given by the relation

$$
a=\frac{1}{1+z}
$$




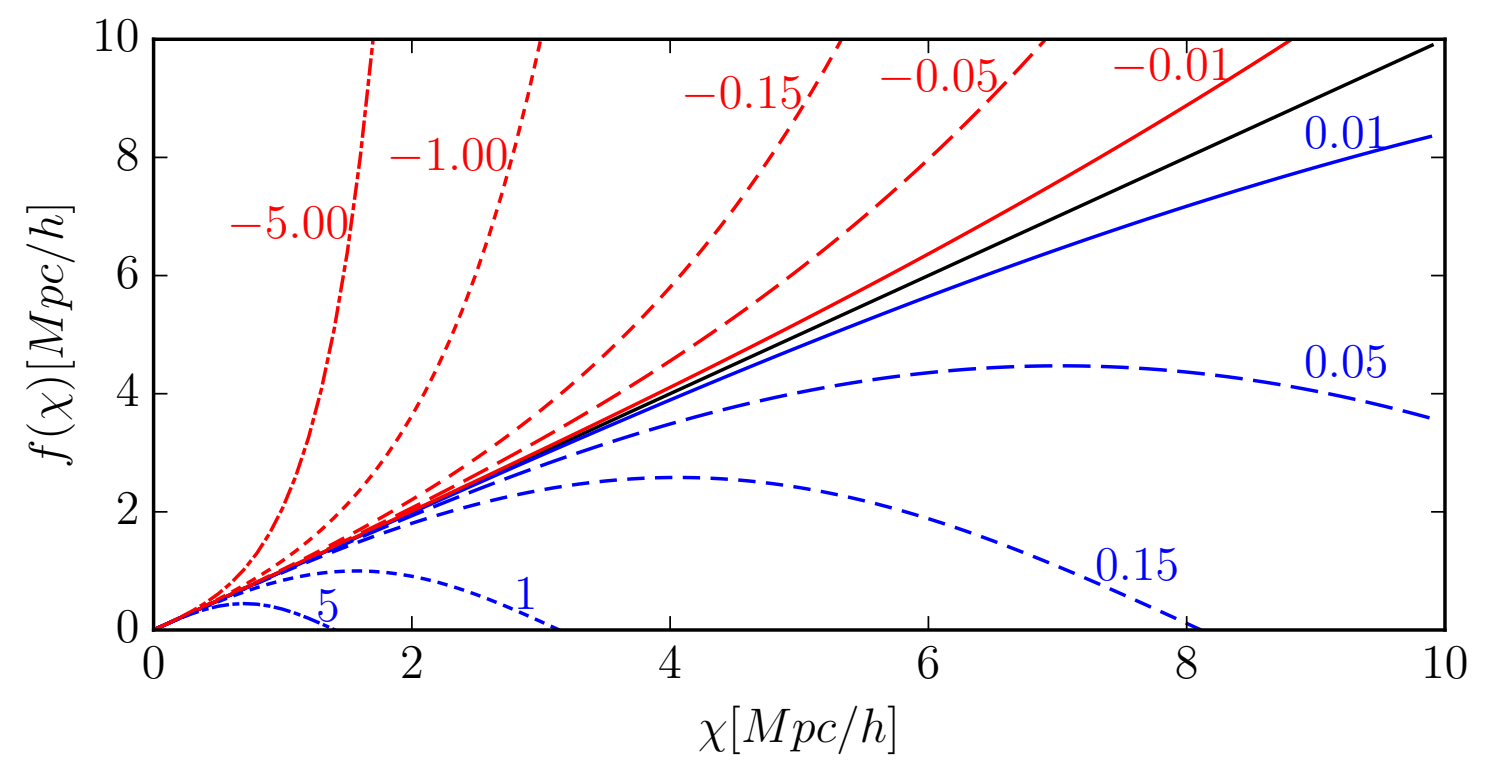

Figure 1.1: Evolution of scales of the Universe for different values of curvature $k$ in units of $M c p^{-2} h^{2}$.

\subsubsection{Hubble Parameter}

The Hubble parameter $H$ measures the relative rate of expansion, and is defined as

$$
H(t)=\frac{\dot{a}}{a}=\frac{1}{a} \frac{d a}{d t}
$$

The value of the Hubble parameter today is called the Hubble constant and denoted by $H\left(t_{0}\right)=$ $H_{0}$. Note that $H_{0}^{-1}$ has units of time and provides roughly (apart from order unit factors) an estimate of the age of the Universe, whereas $c / H_{0}$ gives an estimate of the size of the Universe.

\subsubsection{Friedmann Equations}

Inserting the FRW metric of a homogeneous and isotropic Universe into the Einstein Equations results in the Friedmann's equations

$$
\begin{aligned}
H^{2}(a) & =\frac{8 \pi G}{3} \bar{\rho}_{b k g}(a)+\frac{\Lambda}{3}-\frac{k}{a^{2}} \\
\frac{\ddot{a}}{a} & =-\frac{4 \pi G}{3}\left[\bar{\rho}_{b k g}(a)+3 \bar{P}_{b k g}(a)\right]+\frac{\Lambda}{3}
\end{aligned}
$$

where $\bar{\rho}_{b k g}(a)=\sum \rho_{i}(a)$ and $\bar{P}_{b k g}(a)=\sum P_{i}(a)$ are the total background density and pressure of the Universe at $a$. For a given constitution of the components of the Universe, these equations determine its history and evolution. 
Even though terms related to $\Lambda$ came from the constant of Eq. 1.1 and terms related to $k$ have a geometrical origin, it is possible to treat them like energy components of the universe. By defining the density of the cosmological constant $\Lambda$ as $\rho_{\Lambda} \equiv \Lambda / 8 \pi G$ and of the curvature $k$ as $\rho_{k}(a) \equiv-3 k / 8 \pi G a^{2}$, we can redefine the total density as $\bar{\rho}(a) \equiv \bar{\rho}_{b k g}(a)+\rho_{\Lambda}+\rho_{k}(a)$. Using this density definition in Eq. 1.8 and relating them with $\Lambda$ and $k$ to a pressure of the cosmological constant and curvature results respectively in $P_{\Lambda}=-\Lambda / 8 \pi G$ and $P_{k}=k / 8 \pi G a^{2}$, with the redefined pressure as $\bar{P}=\bar{P}_{b k g}+P_{\Lambda}+P_{k}$. This way, the Friedmann equations can be rewritten as

$$
\begin{aligned}
H^{2}(a) & =\frac{8 \pi G}{3} \bar{\rho}(a) \\
\frac{\ddot{a}}{a} & =-\frac{4 \pi G}{3}[\bar{\rho}(a)+3 \bar{P}(a)]
\end{aligned}
$$

Notice that $\rho_{k}+3 P_{k}=0$, so curvature does not contribute to the second Friedmann equation. Eq. 1.9 shows that the expansion of the universe is dictated by its contents: the Hubble parameter is linked to the amount and behavior of each component of the universe. Introducing the density parameter $\Omega_{i_{0}}(a) \equiv \rho_{i}\left(a_{0}\right) / \rho_{c}\left(a_{0}\right)$, where $\rho_{c}(a) \equiv 3 H^{2} / 8 \pi G$ is defined as the critical density of the universe, the Hubble parameter can be described by

$$
H(a)=H_{0} \sqrt{\sum \Omega_{i_{0}} \frac{\rho_{i}(a)}{\rho_{i_{0}}}} .
$$

Evaluating this equation today, where $\rho_{i}\left(a_{0}\right)=\rho_{i_{0}}$, we have that $\sum \Omega_{i_{0}}=1$. From this relation, it becomes evident that each $\Omega_{i_{0}}$ can be interpreted as the fraction of the respective component today.

The second Friedmann Eq. 1.10, relates the acceleration of the universe with their components. Accelerated expansion requires that $(\bar{\rho}+3 P)<0$. As the energy density $\bar{\rho}$ is always positive, there must be a component with negative pressure resulting in $\bar{P}<-\bar{\rho} / 3$. One possible model involves a dark energy component that satisfies this condition, this model will be discussed in more details in section 1.2 .7 .

We can combine both Friedmann equations by differentiating the first, and have the evolution of the energy density dictated by the Hubble factor as

$$
\dot{\bar{\rho}}+3 H(a)(\bar{\rho}+3 \bar{P})=0
$$


In the case where every component is independent of (does not interact with) each other, this equation has a deeper implication. It means that each $i^{\text {th }}$ component must individually obey the condition in the equation above, i.e. $\dot{\rho}_{i}+3 H(a)\left(\rho_{i}+3 P_{i}\right)=0$.

\subsubsection{Comoving Radial Distance}

The comoving radial distance $\chi$ can be obtained by considering the radial path of photons, in which the angular element $d \alpha=0$ (radial) and also $d s=0$ (photons). From the metric, $\chi$ can be expressed as

$$
\chi=\int_{0}^{\chi} d \chi^{\prime}=\int_{0}^{t} \frac{d t}{a(t)}=\int_{a}^{1} \frac{d a}{\dot{a} a}=\int_{0}^{z} \frac{d z}{H(z)}
$$

Thus $\chi$ can be computed given the Hubble parameter from the Friedmann's equations and depends on the curvature only through the dependence of $H(z)$ on $k$.

\subsubsection{Comoving Angular Diameter Distance}

The comoving angular diameter distance $D_{A}$ is defined such that it gives an objects transverse comoving size $d l$ when it is multiplied by the object angular size $d \alpha$

$$
d l=D_{A} d \alpha
$$

Differently from the comoving radial distance, the comoving angular diameter distance depends explicitly on the spatial curvature. The angular size of objects at cosmological distances is deformed compared to the size in Euclidean geometry. Using the metric in radial coordinates as in Eq. 1.2, the comoving angular diameter distance is precisely the factor that affects the angular term $d \alpha$

$$
D_{A}=f(\chi) \Rightarrow \frac{d D_{A}}{\sqrt{1-k D_{A}^{2}}}=d \chi
$$

Using the equality above, the metric can be written in terms of the comoving angular diameter 
distance

$$
d s^{2}=d t^{2}-a^{2}(t)\left[d \chi^{2}+D_{A}^{2} d \alpha^{2}\right]=d t^{2}-a^{2}(t)\left[\frac{d D_{A}^{2}}{1-k D_{A}^{2}}+D_{A}^{2} d \alpha^{2}\right]
$$

\subsubsection{Comoving Volume}

The comoving volume element in spherical coordinates is given by

$$
d V(z)=\left(D_{A} d \theta\right)\left(D_{A} \sin \theta d \phi\right) d \chi=\frac{D_{A}^{2}(z)}{H(z)} d z d \Omega
$$

where $D_{A}(z)$ is the comoving angular diameter distance at redshift $z, H(z)$ is the Hubble parameter and $d \Omega=\sin \theta d \theta d \phi$ is the solid angle.

\subsection{7 $\Lambda$ CDM - The Standard Cosmological Model}

At present, the $\Lambda$ Cold Dark Model $(\Lambda \mathrm{CDM})$ is the standard cosmological model used to describe the Universe, compatible with nearly every observation made to date. In this model, the universe is composed mainly by dark energy $\Lambda$ and dark matter, with small amounts of baryonic matter and radiation. All the components of this model are considered independent and can be described by equations of states $w_{i}$ such that $p_{i}=w_{i} \rho_{i}$. Using this relation in Eq. 1.12, we can find an analytic solution to the evolution of the density of each component as:

$$
\rho_{i}(a)=\rho_{i_{0}} a^{-3\left(1+w_{i}\right)}=\rho_{i_{0}}(1+z)^{3\left(1+w_{i}\right)}
$$

where $\rho_{i_{0}} \equiv \rho_{i}\left(a_{0}\right)$. In this context, the Hubble parameter can then be described by

$$
H(z)=H_{0} \sqrt{\sum \Omega_{i_{0}}(1+z)^{3\left(1+w_{i}\right)}} .
$$

From Eq. 1.19, it is clear that the the Universe expansion evolution depends crucially on the values of $w_{i}$ and $\Omega_{i_{0}}$ for each component.

According the definitions of effective density and pressure for $\Lambda$ and $k$ in section 1.2 .3 , we have that $w_{\Lambda}=-1$ and $w_{k}=-1 / 3$. The dark energy $\Lambda$ has a negative pressure and is responsible for the accelerated expansion of the Universe. Without dark energy it is not possible to have an accelerated expansion in the GR context. Due the unbeknownst nature of the dark energy, several models have also tried to explain cosmic acceleration with a modification of 


\begin{tabular}{lc||cc}
\hline \hline$\Omega_{m}$ & $0.315 \pm 0.017$ & $h$ & $0.673 \pm 0.012$ \\
$\Omega_{\Lambda}$ & $0.685 \pm 0.017$ & $n_{S}$ & $0.9603 \pm 0.0073$ \\
$\Omega_{R}$ & $9.24 \times 10^{-5}\left(\right.$ from $\left.T_{0}, h\right)$ & $\tau$ & $0.089 \pm 0.013$ \\
$\Omega_{k}$ & $0.000 \pm 0.005$ & $\sigma_{8}$ & $0.829 \pm 0.012$ \\
\hline \hline
\end{tabular}

Table 1.1: Main cosmological parameters from Planck Collaboration et al. (2016).

GR. However, most of these models either have conflict with other well solved problems in GR or present solutions almost indistinguishable from GR. At present, the standard $\Lambda$ CDM model remains as the simplest model that is able to describe all observations to date. Because the total contribution of dark energy (as a cosmological constant) $\rho_{\Lambda}+P_{\Lambda}$ is null, its energy density is constant. This means that as the Universe expands, more dark energy is created such that its energy density does not change. For this reason, dark energy as a cosmological constant can be interpreted as "vacuum" energy, although attempts to compute its value from first principles within Quantum Field Theory have not been successful.

Most of the dark matter is described as "cold", i.e. it was non-relativistic at the time of radiation decoupling, and behaves as dust, where the pressure is negligible compared with the energy density and resulting in $w_{m}=0$. This relation can also be derived by assuming that the total energy of non-relativistic matter is dominated by its rest mass and is therefore constant $\left(\int d V \rho_{m}=\right.$ const $)$. As the Universe expands, the density is simply re-scaled by the inverse volume as $a^{-3}$. Using this relation in Eq. 1.12 results in $P_{m}=0$. The total matter field is composed of baryonic matter, the regular well-known matter, and dark matter, a component with unknown properties and a contribution to the Universe energy density about 5 times larger than that of baryons. The pressure of radiation and ultra-relativistic matter can be described by $P_{r}=\rho_{r} / 3$ leading to $w_{r}=1 / 3$. The resulting expansion of the universe can be described using Eq. 1.19 as

$$
H(z)=H_{0} \sqrt{\Omega_{\Lambda}+\Omega_{k}(1+z)^{2}+\Omega_{m}(1+z)^{3}+\Omega_{r}(1+z)^{4}}
$$

Measurements from Planck Collaboration et al. (2016) report the most recent widely accepted values of all the components. It is convenient to report the main cosmological parameters constrained by the Planck mission in Table 1.1, as they will be used in several sections throughout this thesis.

In Fig. 1.2, we show the evolution of the Hubble factor with redshift. The black solid line 


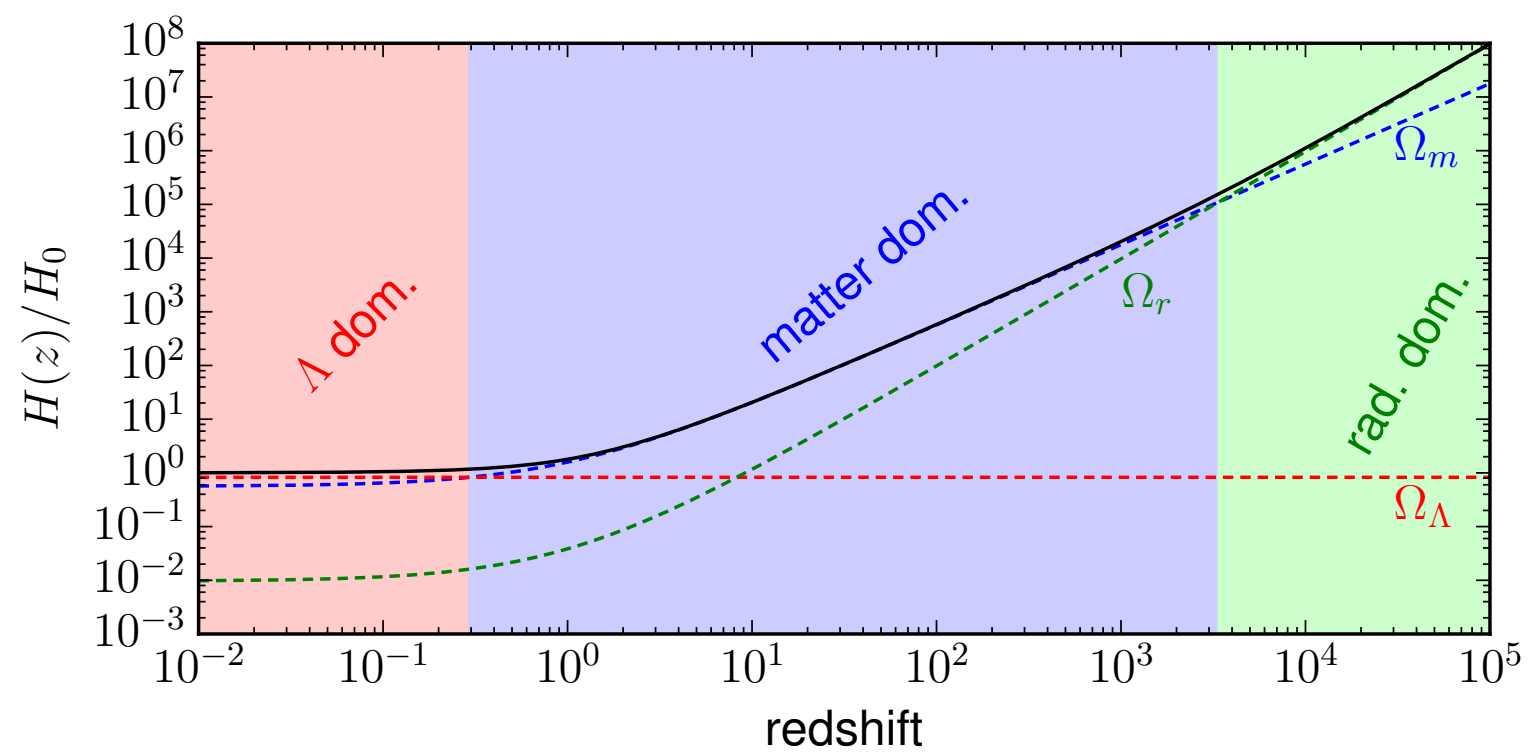

Figure 1.2: Hubble factor evolution with redshift using the Planck cosmology (Table 1.1). The solid line is the Hubble factor with all components, and the dashed lines are the contribution of each component.

is the total value of the Hubble parameter. The blue, green and red dashed lines are the contributions of matter, radiation and dark energy, respectively. In the past, at high redshifts $z \gtrsim 3400$, the expansion was dominated by the evolution of the radiation. At intermediate redshifts $0.3 \lesssim z \lesssim 3400$ matter dominated and today $z \lesssim 0.3$ the Universe is dominated by dark energy, which drives cosmic acceleration.

\subsubsection{Power Spectrum and Density Variance}

In this section we consider a statistically homogeneous and isotropic background with matter density $\bar{\rho}_{m}$, and a local density $\rho(\boldsymbol{x})$ that changes spatially. Defining the overdensity $\delta \rho(\boldsymbol{x})=$ $\rho(x)-\bar{\rho}_{m}$, the density contrast $\delta(\boldsymbol{x})$ is

$$
\delta(x)=\frac{\delta \rho(x)}{\bar{\rho}_{m}}
$$

The two-point correlation function $\xi(r)$ is defined as the ensemble average of the product of $\delta(x)$ at two distinct positions,

$$
\xi(r) \equiv\langle\delta(\boldsymbol{x}) \boldsymbol{\delta}(\boldsymbol{x}+\boldsymbol{r})\rangle
$$

which depends only upon $r \equiv\|\boldsymbol{r}\|$ due to the statistical homogeneity and isotropy. In Fourier 
space, the overdensity field is

$$
\tilde{\boldsymbol{\delta}}(\boldsymbol{k})=\int \mathrm{d}^{3} \boldsymbol{x} \delta(\boldsymbol{x}) e^{i \boldsymbol{k} \cdot \boldsymbol{x}}
$$

and the inverse relation is given by

$$
\boldsymbol{\delta}(\boldsymbol{x})=\int \frac{\mathrm{d}^{3} \boldsymbol{k}}{(2 \pi)^{3}} \tilde{\boldsymbol{\delta}}(\boldsymbol{k}) e^{-i \boldsymbol{k} \cdot \boldsymbol{x}}
$$

The overdensity field is evidently real, which means $\delta(x)=\delta^{*}(\boldsymbol{x})$. Thus, inserting Eq. 1.24 in Eq. 1.22 and defining the power spectrum $P(k)$ via

$$
\left\langle\tilde{\boldsymbol{\delta}}(\boldsymbol{k}) \tilde{\boldsymbol{\delta}}^{*}\left(\boldsymbol{k}^{\prime}\right)\right\rangle \equiv(2 \pi)^{3} \boldsymbol{\delta}_{D}\left(\boldsymbol{k}-\boldsymbol{k}^{\prime}\right) P(k)
$$

where $\delta_{D}(k)$ is the Dirac delta function, the two-point correlation function can be computed as

$$
\xi(r)=\int \frac{\mathrm{d}^{3} \boldsymbol{k}}{(2 \pi)^{3}} P(k) e^{-i \boldsymbol{k} \cdot \boldsymbol{r}}=\frac{4 \pi}{(2 \pi)^{3}} \int d k k^{2} P(k) \frac{\sin (k r)}{k r}
$$

The angular part of Eq. 1.26 was integrated at the last step, since $P(k)$ depends only on $k=|\boldsymbol{k}|$ due to isotropy.

For $r=0$, the correlation function becomes the variance of the density field, $\sigma^{2}=\xi(r=0)$, and can be expressed as

$$
\sigma^{2}=\frac{1}{2 \pi^{2}} \int d k k^{2} P(k)=\int \frac{d k}{k}\left(\frac{k^{3} P(k)}{2 \pi^{2}}\right) .
$$

An interesting statistical quantity is

$$
\Delta^{2} \equiv \frac{k^{3} P(k)}{2 \pi^{2}}
$$

which measures the power per logarithmic scale. Scales in which non-linear effects become important are those in which $\Delta \gtrsim 1$.

For a Gaussian density field, the power spectrum contains all relevant statistical information. However, gravitational collapse causes an initially Gaussian distribution to develop a skewness. That happens because $\langle\delta(\boldsymbol{x})\rangle=0$ and, although $\delta(\boldsymbol{x})$ may assume arbitrarily large values (e.g. inside galaxy clusters, black holes, etc.), it has a minimum value of -1 inside voids. Deviations 
from gaussianity can be characterized by higher order correlations (e.g. 3-point correlations) and their counterparts in Fourier space (bi-spectrum, etc.).

The matter power spectrum can be calculated in linear theory by evolving the coupled Einstein-Boltzmann equations (Ma \& Bertschinger, 1995) for the various components in the Universe. Advanced and highly tested codes such as CMBFAST (Seljak \& Zaldarriaga, 1996; Zaldarriaga et al., 1998; Zaldarriaga \& Seljak, 2000) and CAMB (Lewis et al., 2000) make this calculation fast and precise. In Fig. 1.3 we show the power spectrum computed using CAMB the cosmology from Planck Collaboration et al. (2016).

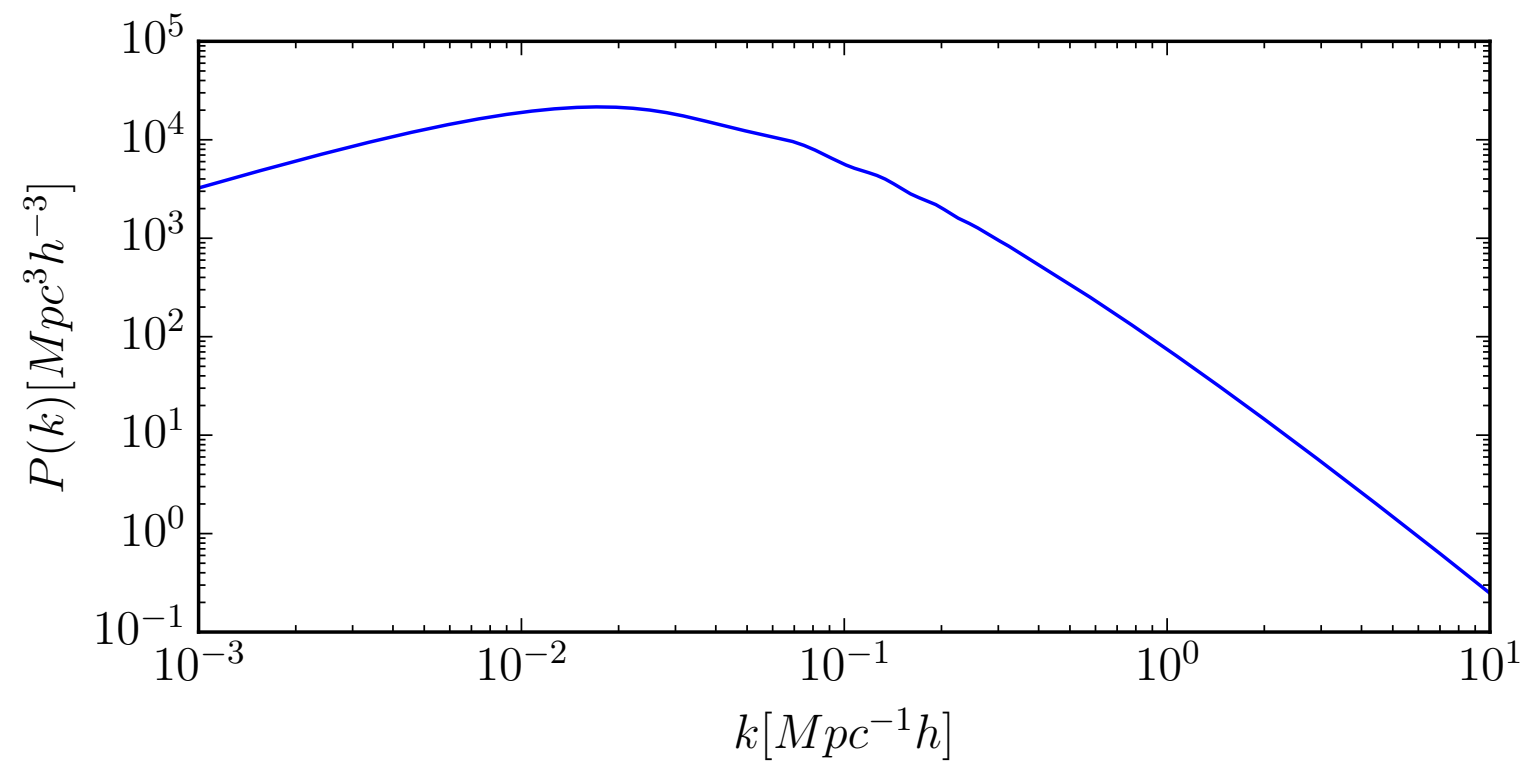

Figure 1.3: Matter power spectrum with Planck Collaboration et al. (2016) cosmology.

\subsubsection{Linear Growth Function}

The evolution of the dark matter density field is described by the coupled Einstein-Boltzmann equations mentioned before. To compute the linear growth, these equations are equivalent to a simpler approach using perturbation theory in fluid mechanics. The hydrodynamical conservation equations, such as the continuity and Euler equations as well as the Poisson equation for a pressureless fluid are

$$
\begin{aligned}
\frac{\partial \rho}{\partial t}+\nabla \cdot(\rho \boldsymbol{v}) & =0, \\
\frac{\partial \boldsymbol{v}}{\partial t}+(\boldsymbol{v} \cdot \nabla) \boldsymbol{v} & =-\nabla \phi, \\
\nabla^{2} \boldsymbol{\phi} & =4 \pi G \rho .
\end{aligned}
$$


In this case it is convenient to use the cosmological comoving frame for the calculations, through the following well-known transformations (Peebles, 1980):

$$
\begin{aligned}
\nabla & \rightarrow \frac{1}{a} \nabla \\
\frac{\partial f}{\partial t} & \rightarrow \frac{\partial f}{\partial t}-H \boldsymbol{r} . \nabla f
\end{aligned}
$$

where $a$ is the scale factor, $H$ the Hubble parameter and $r$ the radial coordinate in the FriedmannRobertson-Walker metric. Note that the fluid velocity is the velocity of the expansion of the Universe: $\boldsymbol{v} \equiv \dot{a} \boldsymbol{r}$.

By introducing the following perturbations in the velocity, density and gravitational potential:

$$
\boldsymbol{v}=\dot{a} \boldsymbol{r}+\boldsymbol{u}, \quad \rho=\bar{\rho}+\delta \rho, \quad \phi=\bar{\phi}+\phi^{\prime},
$$

and changing to the comoving frame, Eqs. 1.29, 1.30 and 1.31 in linear theory can be expressed as:

$$
\begin{aligned}
\frac{\partial \delta}{\partial t}+\frac{1}{a} \nabla \cdot(\boldsymbol{u}) & =0 \\
\frac{\partial \boldsymbol{u}}{\partial t}+H \boldsymbol{u} & =-\frac{1}{a} \nabla \phi \\
\nabla^{2} \phi^{\prime} & =4 \pi G a^{2} \bar{\rho} \delta
\end{aligned}
$$

where $\delta \equiv \delta \rho / \bar{\rho}$ is once again the density contrast. Here terms of second order and above in the perturbations have been neglected.

Combining the time derivative of Eq. 1.35, the divergence of Eq. 1.36, and Eq. 1.37, produces a single equation that describes the time evolution of the density contrast:

$$
\ddot{\delta}+2 H \dot{\delta}=\frac{3}{2} \frac{\Omega_{m} H_{0}^{2}}{a^{3}} \delta,
$$

where $\dot{\delta} \equiv \partial \delta / \partial t$. It is interesting to have the equation for the evolution of $\delta$ with respect to the scale factor. Using the chain rule $\partial / \partial t=\dot{a} \partial / \partial a$ and the notation $\delta^{\prime} \equiv \partial \delta / \partial a$, Eq. 1.38 can be rewritten as:

$$
\delta^{\prime \prime}+\left(\frac{H^{\prime}}{H}+\frac{3}{a}\right) \delta^{\prime}=\frac{3}{2} \frac{\Omega_{m} H_{0}^{2}}{a^{5} H^{2}} \delta .
$$





Figure 1.4: Linear growth function versus redshift for different cosmological models. Left: flat $\Lambda$ CDM model. Right: flat $w \mathrm{CDM}$ model with $\Omega_{m}=0.25$.

After Fourier transforming the above equation, its solution can be expressed as $\delta(k, z)=$ $G(z) \delta(k, z=0)$, which relates explicitly the evolution of the density perturbation field with $G(z)$, the linear growth function. This effectively separates the coordinates $k$ from the time or redshift, such that $G(z)$ satisfies the same equation above while the spatial dependence is all contained in $\delta(k, z=0)$. Furthermore, the same separation can be used on the power spectrum of the dark matter field, such that the redshift dependence is carried on by the growth function

$$
P(k, z)=G^{2}(z) P(k, z=0)
$$

Likewise, the growth function $G(z)$ provides the redshift dependence for several cosmological quantities in linear theory. In Fig. 1.4, the growth function is shown for different values of $\Omega_{m}$ and $w$. As the growth function is normalized to its value today, so that $G(z=0)=1$.

In the case of a Universe with only matter $\left(\Omega_{m}=1\right)$, the exact solution is $H(a)=H_{0} a^{-3 / 2}$, therefore Eq. 1.39 can be written as:

$$
\delta^{\prime \prime}+\frac{3}{2 a} \delta^{\prime}-\frac{3}{2 a^{2}} \delta=0
$$

which also has an exact solution $\delta=A a+B a^{-3 / 2}$. Using the evolution of the scale factor in a $C D M$ Universe $\left(a^{3 / 2}(t)=3 H_{0} t / 2\right)$, one can obtain a time solution for the growth function:

$$
\delta=A t^{2 / 3}+B t^{-1}
$$


The first term in Eq. 1.42 corresponds to the growing mode $\delta=a$ which dominates the evolution, while the second term is the decaying mode $\delta=H$. 


\section{Chapter 2}

\section{Halo Abundance Formalism}

In this chapter we will present the formalism used to study the formation and abundance of dark matter halos in the Universe. Our approach will be first to understand the theory describing the expected distribution of halos from first principles with some simplifying assumptions. Then we will apply the improvements developed along the years from simulations, which allow us to go beyond these simplifications into more realistic predictions. Additionally, we will study the halo bias, caused by the use of collapsed objects to trace the dark matter density field. Finally, we will inspect the covariance of halo counts that will ultimately predict the uncertainty of the halo prediction. These theoretical elements will later be used within likelihood methods for constraining cosmology with galaxy clusters.

\subsection{Spherical Collapse}

The spherical collapse model is a simplified description of the non-linear evolution that leads to structure formation and, in particular, to the formation of dark matter halos, which decouple from the Hubble expansion and collapse gravitationally.

Even though it is a rather simple model, it is able to provide intuition on the very complex non-linear dynamics of gravitational collapse. It even allows for the quantitative computation of interesting quantities, such as the collapse density extrapolated in linear theory $\delta_{c}-$ useful in the Press-Schechter formalism of halo abundance - and the virial overdensity $\Delta_{\text {vir }}-$ useful to provide a natural threshold to define virialized dark matter halos and galaxy clusters.

In its simplest version, the spherical collapse model considers an isolated spherical top-hat matter overdensity $\delta$, embedded in a background with the average density of the Universe. This 
overdensity is then treated using perturbation theory in fluid mechanics for a pressureless fluid:

$$
\begin{aligned}
\frac{\partial \delta}{\partial t}+\frac{1}{a} \nabla .(1+\delta) \boldsymbol{u} & =0 \\
\frac{\partial \boldsymbol{u}}{\partial t}+\frac{1}{a}(\boldsymbol{u} . \nabla) \boldsymbol{u}+H \boldsymbol{u} & =-\frac{1}{a} \nabla \phi^{\prime} \\
\nabla^{2} \phi^{\prime} & =4 \pi G a^{2} \bar{\rho} \delta .
\end{aligned}
$$

It is important to note that, in this case, there are extra non-linear terms, not present in the linear theory case considered for the growth function. Once again, combining the time derivative of the continuity equation (Eq. 2.1) and the divergence of Euler equation (Eq. 2.2), we obtain a single equation that gives us the time evolution of the density contrast

$$
\ddot{\boldsymbol{\delta}}+2 H \dot{\boldsymbol{\delta}}-\frac{1}{a^{2}} \nabla \cdot\{[\nabla \cdot(1+\boldsymbol{\delta}) \boldsymbol{u}] \boldsymbol{u}+(1+\boldsymbol{\delta})(\boldsymbol{u} \cdot \nabla) \boldsymbol{u}\}=\frac{1}{a^{2}} \nabla \cdot\left[(1+\boldsymbol{\delta}) \nabla \phi^{\prime}\right]
$$

As we assumed a top-hat overdensity, the value of $\delta$ is spatially constant inside the collapsing region. Additionally, the top-hat preserves the spatial homogeneity throughout the evolution, requiring a velocity proportional to the radius $\boldsymbol{u} \propto \boldsymbol{r}(\nabla . \boldsymbol{u}=3 \partial u / \partial r)$. Hence, we can simplify the term

$$
\begin{aligned}
\nabla .\{[\nabla .(1+\delta) \boldsymbol{u}] \boldsymbol{u}+(1+\delta)(\boldsymbol{u} \cdot \nabla) \boldsymbol{u}\} & =(1+\boldsymbol{\delta})[\nabla \cdot(\nabla \cdot \boldsymbol{u}) \boldsymbol{u}+\nabla \cdot(\boldsymbol{u} \cdot \nabla) \boldsymbol{u}] \\
=\frac{4}{3}(1+\delta)(\nabla \cdot \boldsymbol{u})^{2} & =\frac{4}{3} \frac{a^{2} \dot{\boldsymbol{\delta}}^{2}}{1+\delta}
\end{aligned}
$$

where Eq. 2.1 was used once again. Therefore, the perturbation equation can be written without explicit dependency on the velocity $u$

$$
\ddot{\delta}+2 H \dot{\delta}-\frac{4}{3} \frac{\dot{\delta}^{2}}{1+\delta}=\frac{1}{a^{2}}(1+\delta) \nabla^{2} \phi^{\prime}
$$

During collapse, the total mass of the perturbation is conserved, and we have $\bar{\rho}_{m}(1+\delta) \propto$ $r^{-3}$. Inserting time derivatives of this relation combined in Eq. 2.6, the evolution of the perturbation can be analyzed as a function of its radius

$$
\frac{\ddot{r}}{r}=\frac{\ddot{a}}{a}-\frac{1}{3 a^{2}} \nabla^{2} \phi^{\prime}
$$


Using the Friedmann equation (Eq. 1.10) and the results of Poison equation (Eq. 2.3) in the evolution of the perturbation (Eq. 2.7), we find how the evolution of the perturbation radius is related with its density the content of the background Universe

$$
\begin{aligned}
\frac{\ddot{r}}{r} & =-\frac{4 \pi G}{3}\left[\bar{\rho}_{m}+\sum_{i} \bar{\rho}_{i}+3 \bar{P}_{i}\right]-\frac{4 \pi G}{3} \bar{\rho}_{m} \delta \\
& =-\frac{4 \pi G}{3}\left[\rho_{m}+\sum_{i} \bar{\rho}_{i}+3 \bar{P}_{i}\right] .
\end{aligned}
$$

For the case of $w C D M$ the equation for the growth of the collapse is simplified to

$$
\frac{\ddot{r}}{r}=-\frac{4 \pi G}{3}\left[\rho_{m}+(1+3 w) \bar{\rho}_{D E}\right]
$$

and does not have an analytic solution in general.

The particular case of a universe containing only Dark Matter has an exact solution for $r(t)$. Solving the Friedmann equation leads to a scale factor growing as $a(t) \propto t^{2 / 3}$ and a respective background density $\bar{\rho}_{m}=1 /\left(6 \pi G t^{2}\right)$. In this case, Eq. 2.9 results in a simple equation for the size of the perturbations:

$$
\ddot{r}=-\frac{G M}{r^{2}}
$$

This equation can be integrated, producing:

$$
\dot{r}^{2}=\frac{2 G M}{r}-c
$$

with an arbitrary constant $c$. Here it can be seen that the size of the perturbations is limited $(\dot{r}=0)$ for physical solutions, i.e. positive values of $c$. Furthermore, a valid solution implies that the maximum value of the radius must be $r_{\max }=2 G M / c$, since the LHS of Eq. 2.11 must be positive. Another way to see this is to interpret this equation as giving the total energy per unit mass $E$ of a gravitational system having $E=-c / 2$ :

$$
E=\frac{\dot{r}^{2}}{2}-\frac{G M}{r}
$$

The system is analog to a particle that is only "trapped" by gravity if the total energy is negative. This particular differential equation has the cycloid as the implicit solution in terms of a 
development angle $\theta$

$$
\begin{aligned}
& r=\left(\frac{G M}{c}\right)(1-\cos \theta) \\
& t=\left(\frac{G M}{c^{3 / 2}}\right)(\theta-\sin \theta) .
\end{aligned}
$$

Therefore, the linear approximation of $r$ is:

$$
r \approx \frac{\sqrt[3]{G M}}{2}(6 t)^{2 / 3}\left[1-\frac{1}{20}\left(\frac{6 c^{3 / 2} t}{G M}\right)^{2 / 3}\right]
$$

and the linear extrapolated value of $\delta$ can be computed using the background density $\left(\bar{\rho}_{m}=\right.$ $1 / 6 \pi G t^{2}$ ) with the mass definition $M=4 \pi r^{3} \bar{\rho}_{m}(1+\delta) / 3$

$$
(1+\delta)=\frac{3 M}{4 \pi} \frac{1}{r^{3} \bar{\rho}_{m}}=\frac{1}{\left[1-\frac{1}{20}\left(\frac{6 c^{3 / 2} t}{G M}\right)^{2 / 3}\right]^{3}} \approx 1+\frac{3}{20}\left(\frac{6 c^{3 / 2} t}{G M}\right)^{2 / 3}
$$

resulting in

$$
\delta \approx \frac{3}{20}\left(\frac{6 c^{3 / 2} t}{G M}\right)^{2 / 3}
$$

Collapse occurs at $\theta=2 \pi \rightarrow t_{c}=2 \pi G M / c^{3 / 2}$, and the linear extrapolation of the density perturbation at the time of collapse is

$$
\delta_{c}=\frac{3}{20}(12 \pi)^{2 / 3} \approx 1.686
$$

This quantity $\delta_{c}$ can be interpreted as a threshold density, for which overdensities $\delta>\delta_{c}$ become dark matter halos. Another important moment of the collapse process occurs at virialization $(U=-2 K)$, where, from Eq. 2.12, $\dot{r}_{\text {vir }}^{2}=G M / r_{\text {vir }}$. According to Eq. 2.13, virialization must happen for $\cos (\theta)=0$. This leads to $\theta_{v i r}=3 \pi / 2$, since it must happen before collapse. As a consequence, the overdensity of the perturbation $(\Delta=1+\delta)$ at virialization is

$$
\Delta_{\mathrm{vir}}=\frac{M}{\frac{4}{3} \pi r_{\mathrm{vir}}^{3} \bar{\rho}_{m}\left(t_{\mathrm{vir}}\right)}=18 \pi^{2} \approx 178 .
$$


This means that, at virialization, the overdensity of the perturbation is roughly 180 times the background density. In the case of a $\Lambda \mathrm{CDM}$ cosmology, the solution of Eq.2.9 can be obtained numerically, resulting in a different overdensity threshold and virialization overdensity according to the amount of dark matter and dark energy. However, the CDM values of $\delta_{c}(1.686)$ and $\Delta_{\text {vir }}(178)$ are generally adopted as useful references.

\subsection{Filtering and Window Function}

In the galaxy cluster framework, the perturbations on the dark matter field are traced by collapsed objects, the dark matter halos. It will be useful to consider not the pure matter field $\delta(\boldsymbol{x})$ per se, but the field $\delta_{R}(\boldsymbol{x})$, which is filtered by a window-function $W(\boldsymbol{x}, R)$ associated to a scale $R$. Mathematically, the field $\delta_{R}(\boldsymbol{x})$ is given by the convolution of $\delta(\boldsymbol{x})$ and $W(\boldsymbol{x}, R)$, i.e.

$$
\delta_{R}(\boldsymbol{x})=\int \mathrm{d}^{3} \boldsymbol{x} \delta(\boldsymbol{x}) W(\boldsymbol{x}, R)
$$

The simplest window is the radial Top-Hat, which defines a spherical volume of constant density with a fixed radius $R$ :

$$
W(x, R)=\left\{\begin{array}{cc}
1 & \text { if } r<R \\
0 & \text { if } r>R
\end{array},\right.
$$

where $r=|\boldsymbol{x}|$. There are more sophisticated window-functions such as Gaussian and Sharp $k$ space Lacey \& Cole (1994), however, these profiles lead to a much more complicated formalism and will not be in the scope of this work. Using $\delta_{R}$ instead of $\delta$ in the previous definitions (Eq. 1.27), the filtered overdensity field variance $\sigma(R)=\xi(r=0, R)$ becomes

$$
\sigma^{2}(R)=\frac{1}{2 \pi^{2}} \int d k k^{2} P(k) \tilde{W}^{2}(k R)
$$

where $\tilde{W}(y)$ is the Fourier transform of the window function. For the Top-Hat window function,

$$
\tilde{W}(y)=\frac{3}{y^{2}}\left(\frac{\sin y}{y}-\cos y\right) .
$$

This variance can also be expressed as a function of the background mass $M=4 \pi R^{3} \bar{\rho}_{m} / 3$ contained in a sphere of radius $R$, i.e. $\sigma(M)=\sigma\left(R=\left[3 M / 4 \pi \bar{\rho}_{m}\right]^{1 / 3}\right)$. Additionally, the density 
variance also has a dependence on redshift via the power spectrum. Using the separation of the redshift dependence from Eq. 1.40 , the variance at any redshift can be computed by $\sigma(z, R)=$ $G(z) \sigma(z=0, R)$, where $\sigma(z=0, R)$ is calculated with the linear power spectrum at $z=0$.

\subsection{Halo Mass-function}

Dark matter halos are collapsed regions within the dark matter field. From the spherical collapse model, they were formed in regions where the overdensity was $\delta>\delta_{c}$, which eventually collapsed and virialized. Press \& Schechter (1974) estimated the fraction of matter within dark matter halos $F(M)$ with masses larger than $M$, considering density fluctuations in Fourier space $\delta(k)$, which perform a random walk as we consider different smoothing scales $R$. Therefore, in the absence of a non-Gaussian primordial source of perturbations, the distribution is Gaussian and the fraction of matter inside dark matter halos can be computed as

$$
F(M)=\frac{1}{\sqrt{2 \pi} \sigma(M)} \int_{\delta_{c}}^{\infty} d \delta \exp \left[-\frac{\delta^{2}}{2 \sigma^{2}(M)}\right]=\frac{1}{2} \operatorname{erfc}\left[\frac{\delta_{\mathrm{c}}^{2}}{\sqrt{2} \sigma(\mathrm{M})}\right]
$$

In the equation above, for the limit $\sigma \rightarrow \infty$ only half of the total matter is recovered within halos, as $F \rightarrow 1 / 2$. This happens because only overdense regions with $\delta>\delta_{c}$ were considered in the computation. However, regions with $\delta<\delta_{c}$ and underdense regions also contribute to the formation of halos. To account for this effect, Peacock (1999) suggested a factor of 2 should be added to the prediction. Then the differential fraction of halos with masses in the range $[\ln M, \ln M+d \ln M]$ becomes

$$
\frac{d F}{d \ln M}=2 \frac{d F}{d \sigma} \frac{d \sigma(M)}{d \ln M}=\sqrt{\frac{2}{\pi}} \frac{\delta_{c}}{\sigma^{2}} \exp \left[-\frac{\delta^{2}}{2 \sigma^{2}(M)}\right] \frac{d \sigma(M)}{d \ln M}
$$

The differential comoving mean halo number density can be calculated multiplying the differential fraction by the comoving matter number density $n \equiv \bar{\rho}_{m} / M$

$$
\frac{d \bar{n}}{d \ln M}=-\frac{\bar{\rho}_{m}}{M} \frac{d F}{d \ln M}=\frac{\bar{\rho}_{m}}{M} \frac{d \ln \sigma^{-1}}{d \ln M} \sqrt{\frac{2}{\pi}} \frac{\delta_{c}}{\sigma} \exp \left[-\frac{\delta_{c}^{2}}{2 \sigma^{2}}\right],
$$

The minus signal of Eq. 2.26 appears because $d F / d M$ is negative (the fraction of matter always decreases as the mass threshold grows), but the number density must be positive. This 
differential number density is generally written as

$$
\frac{d \bar{n}(z, M)}{d \ln M}=\frac{\bar{\rho}_{m}}{M} \frac{d \ln \sigma^{-1}}{d \ln M} f(\sigma),
$$

and represents the differential number of halos of mass $M$ at redshift $z$ per unit $\ln M$ per unit comoving volume. In this representation $f(\sigma)$ is called the multiplicity function while $d \bar{n} / d \ln M$ is the mass function. Different functional forms of the mass function have been reported for different assumptions made, e.g. spherical symmetry or ellipsoidal collapse. It can also be calibrated from N-body simulations for more accurate results. Because all these functional forms of the mass function differ only on the multiplicity function, $f(\sigma)$ is usually referred to as mass function in the literature.

An important issue with the mass function, specially for those calibrated in simulations, is its universality. That is, whether they are valid in all cosmologies, as a limited mass function can lead to a wrong prediction of halos and a consequent biased cosmology. In order to prevent this problem, the mass function is usually fitted in several simulation with different underlying cosmology (Jenkins et al., 2001; Tinker et al., 2008), thus finding the optimal parameters as well as the confidence level of the fit. This confidence level will set a limit of the halo abundance prediction for each mass function.

Another issue with the calibration of mass functions in simulations is the influence of the baryonic matter. To introduce baryon in dark matter simulations is an extremely complex problem as it not only involves simulating processes of very different scales, but also requires working in non-linear scales with astrophysical origin. Hence, the calibration of mass functions occur on dark matter simulation only (Jenkins et al., 2001; Tinker et al., 2008). Consequently, this is an extensive problem that is still in development (Cui et al., 2012; Bocquet et al., 2016) and will not be evaluated in the scope of this work.

A relevant feature of the mass function, is that the term $f(\sigma) d \ln \sigma^{-1} / d \ln M$ corresponds to the fraction of matter in the range $[\ln M, \ln M+d \ln M]$, hence integrating it over all masses should result in unity. Consequently, a condition that can be imposed to the different functional forms of the mass function is

$$
\int f(\sigma) d \ln \sigma^{-1}=1
$$


Even though this condition is imposed by the definition of the mass function, and it is relevant to the halo bias computations (see Sec. 2.5), it may not be necessary from a practical standpoint in some applications. For instance, in the computation of the halo counts in a given mass bin or above a mass threshold, this condition does not have a direct impact. Therefore, some empirical mass function functional forms fitted for this purpose disregard it.

In the next subsections, we will present and discuss briefly a few mass functions present in the literature. The results for the multiple mass functions presented are shown in Fig. 2.1, where we plot $d n / d \ln M$ as a function of halo mass $M$ at $z=0$ and their ratio to the Press-Schechter mass function.

\subsubsection{Press-Schechter Mass-function}

The formalism above was first demonstrated by Press \& Schechter (1974), with the basic assumptions of spherical collapse and using Gaussian initial conditions. Comparing Eq. 2.26 and Eq. 2.27, the Press-Schechter mass function is given by

$$
f(\sigma)=\sqrt{\frac{2}{\pi}} \frac{\delta_{c}}{\sigma} \exp \left[-\frac{\delta_{c}^{2}}{2 \sigma^{2}}\right]
$$

As defined in Eq. 2.25, this mass function is normalized.

\subsubsection{Sheth \& Tormen Mass-function}

Sheth et al. (2001) parameterized the following mass-function considering an ellipsoidal collapse

$$
f(\sigma)=A \sqrt{q \frac{2}{\pi}}\left[1+\left(q \frac{\delta_{c}^{2}}{2 \sigma^{2}}\right)^{-p}\right] \frac{\delta_{c}}{\sigma} \exp \left[-q \frac{\delta_{c}^{2}}{2 \sigma^{2}}\right] .
$$

This functional form provided a good fit to a set of N-body simulations(Jenkins et al. 2001) with parameters set to $A=0.322, q=0.707$ and $p=0.3$, imposing the normalization. Notice that for $A=q=1$ and $p=0$, the Sheth-Tormen mass-function reduces to the Press-Schechter result. Since the fit to simulations produce values considerably different from the Press-Schechter limit, this indicates that spherical collapse alone is unable to describe the abundance of dark matter halos. 


\subsubsection{Jenkins Mass-function}

Derived in 2001 (Jenkins et al., 2001), this mass function was proposed to fit several N-body dark matter simulations better than the Sheth \& Tormen model. The main goal was to obtain a functional form without an explicit dependency on cosmological parameters and epoch, with all this information carried out via the linear power spectrum.

$$
f(\sigma)=a \exp \left[-|b-\ln \sigma|^{c}\right] .
$$

The parameters that produced the best fit were $a \approx 0.3, b \approx 0.6$ and $c \approx 0.38$ with slight differences depending on the methods used for detection of the dark matter halos (via Spherical Overdensity or Friends of Friends - explained in chapter 3.2) and whether the normalization was imposed.

\subsubsection{Tinker Mass-function}

Based on a more recent set of N-body simulations, this fitting form was devised by Tinker et al. (2008) for halos detected via Spherical Overdensity. Considering that trying to fit a universal function for different epochs decreased the accuracy of the predicted number of dark matter halos, the Tinker mass function given by

$$
f(\sigma)=A\left[\left(\frac{\sigma}{b}\right)^{-a}+1\right] \exp \left[-c / \sigma^{2}\right]
$$

has a dependence on redshift for the parameters $A, a, b$ and $c$. These parameters also depend on the value of the overdensity $\Delta$ chosen for halo detection of the spherical halos. Although, this functional form is not normalized, it presented a better fit to the halos in the simulation. Imposing this condition, Tinker et al. (2008) also included a normalizable mass function

$$
g(\sigma)=B\left[\left(\frac{\sigma}{e}\right)^{-d}+\sigma^{-f}\right] \exp \left[-g / \sigma^{2}\right]
$$

More recently, many other mass function fits have been calibrated from multiple simulations. For instance, Hoffmann et al. (2015) refined the parameters of the Tinker mass function, recalibrating them with the MICE simulations (Fosalba et al., 2015b; Crocce et al., 2015; Fosalba et al., 2015a). 

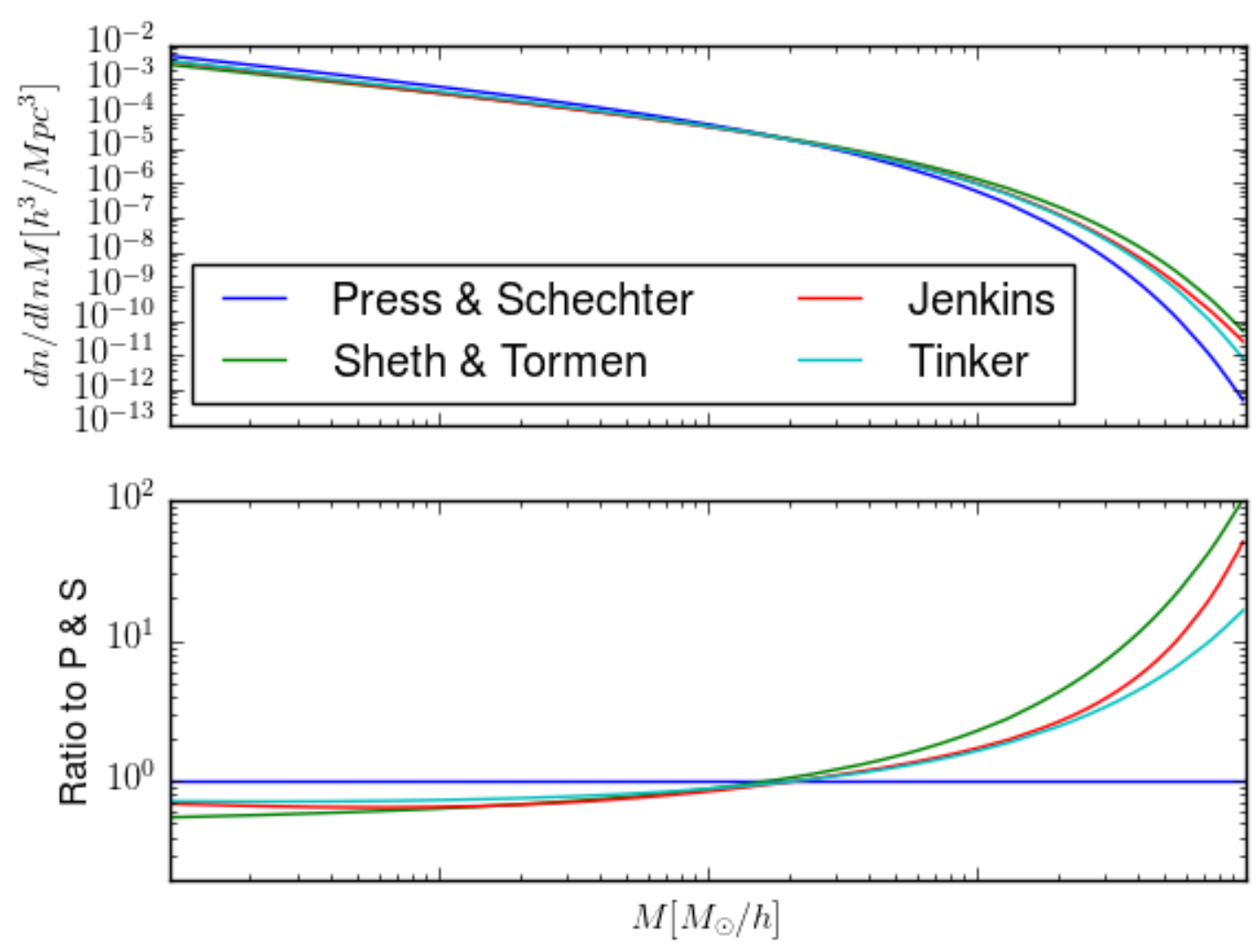

Figure 2.1: Differential comoving mean halo number density as a function of halo mass at $z=0$, for the different mass functions in a $\Lambda C D M$ model. The lower panel shows the ratios of the different mass functions relative to that of Press \& Schechter.

\subsection{Halo Number Counts}

The mean number density of halos in a mass bin $\alpha$ can be calculated integrating Eq. 2.27;

$$
\bar{n}_{\alpha}(z) \equiv \int_{M_{\alpha}}^{M_{\alpha+1}} \frac{d M}{M} \frac{d \bar{n}}{d \ln M}
$$

The mean value of dark matter halo number counts in a redshift bin $i$ and mass bin $\alpha$, results from the integral of Eq. 2.34 in a comoving volume $d V_{i}$ defined by a section of a light cone:

$$
\bar{m}_{\alpha, i}=\int d V_{i} \bar{n}_{\alpha}(z)=\Delta \Omega \int_{z_{i}}^{z_{i+1}} d z \frac{D_{A}(z)^{2}}{H(z)} \bar{n}_{\alpha}(z)
$$

where $\Delta \Omega$ is the total angular size (solid angle) of the survey in $\operatorname{rad}^{2}$ and $\Delta z=z_{i+1}-z_{i}$ is the redshift bin size. 
For a sufficiently small redshift bin $\Delta z=z_{i+1}-z_{i}$, one can approximate:

$$
\bar{m}_{\alpha, i} \approx \Delta V \bar{n}_{\alpha}(z)=\Delta \Omega \Delta z \frac{D_{A}\left(z_{m}\right)^{2}}{H\left(z_{m}\right)} \bar{n}_{\alpha}\left(z_{m}\right)
$$

with $z_{m}=\left(z_{i}+z_{i+1}\right) / 2$.

In Fig. 2.2, the halo number counts were computed for a threshold mass of $10^{13.8} M_{\odot} h^{-1}$ in an area of $\approx 10,313 \mathrm{deg}^{2}$, using the Tinker mass function (section 2.3.4) and a fiducial cosmology with parameters: $\Omega_{m}=0.27, w=-1, \sigma_{8}=0.88, h=0.7, n_{s}=0.96, \Omega_{b}=0.04$. This resulted in $\approx 450 k$ halos. Then each parameter was increased by $20 \%$, while keeping all others fixed. This meant re-scaling the amplitude of the spectrum in order to keep the value of $\sigma_{8}$ fixed.

The increase on $\Omega_{m}$ resulted in a total increase of $23 \%$ in the halo abundance, with a higher influence at low redshifts. A higher matter content causes increases matter clustering and enhance halo formation. When the value of $\sigma_{8}$ is increased, we find an even larger $(+49 \%)$ number of dark matter halos, and also a displacement of the peak of halo abundance from redshift $z=0.65$ to $z=0.75$. These effects make the use of halo number counts a powerful tool to constrain $\sigma_{8}$.

The $20 \%$ increment on $w$ does not change by much the total abundance of halos (decrease of $\approx 2 \%$ ), however, it produces an interesting variation on the number count distribution. For lower redshifts $z \lesssim 1.1$ the increase on $w$ decreases the halo abundance, and for $z>1.1$ it increases the counts. This dual behavior makes the correlation of $w$ with the other cosmological parameters depend on the redshift range surveyed, as we will show in section 5.2. One might expect that the abundance of clusters would increase with an increment on $w$, as the dark energy becomes less negative, acting less against gravitational attraction, and consequently structure formation. In fact $n_{\alpha}(z)$ does increase with the increment on $w$, however, the volume defined by a fixed redshift interval shrinks. The final influence on the halo abundance will depend on the ratio of these two components, both depending on redshift. Hence, the increment on $w$ causes the different changes on the number counts at different redshifts.

The parameters $h$ and $\Omega_{b}$ cause a smaller monotonic changes in the halo number counts ( $7 \%$ and $-2 \%$ respectively), therefore, they will probably not be very well constrained from galaxy cluster abundance measurements alone. Fortunately, most parameters are already well constrained at percent level by CMB measurements. The exception is for dark energy parameters, whose strongest constraints should come from low redshift structure measurements (e.g. 
cluster counts) in combination with high redshift CMB measurements.

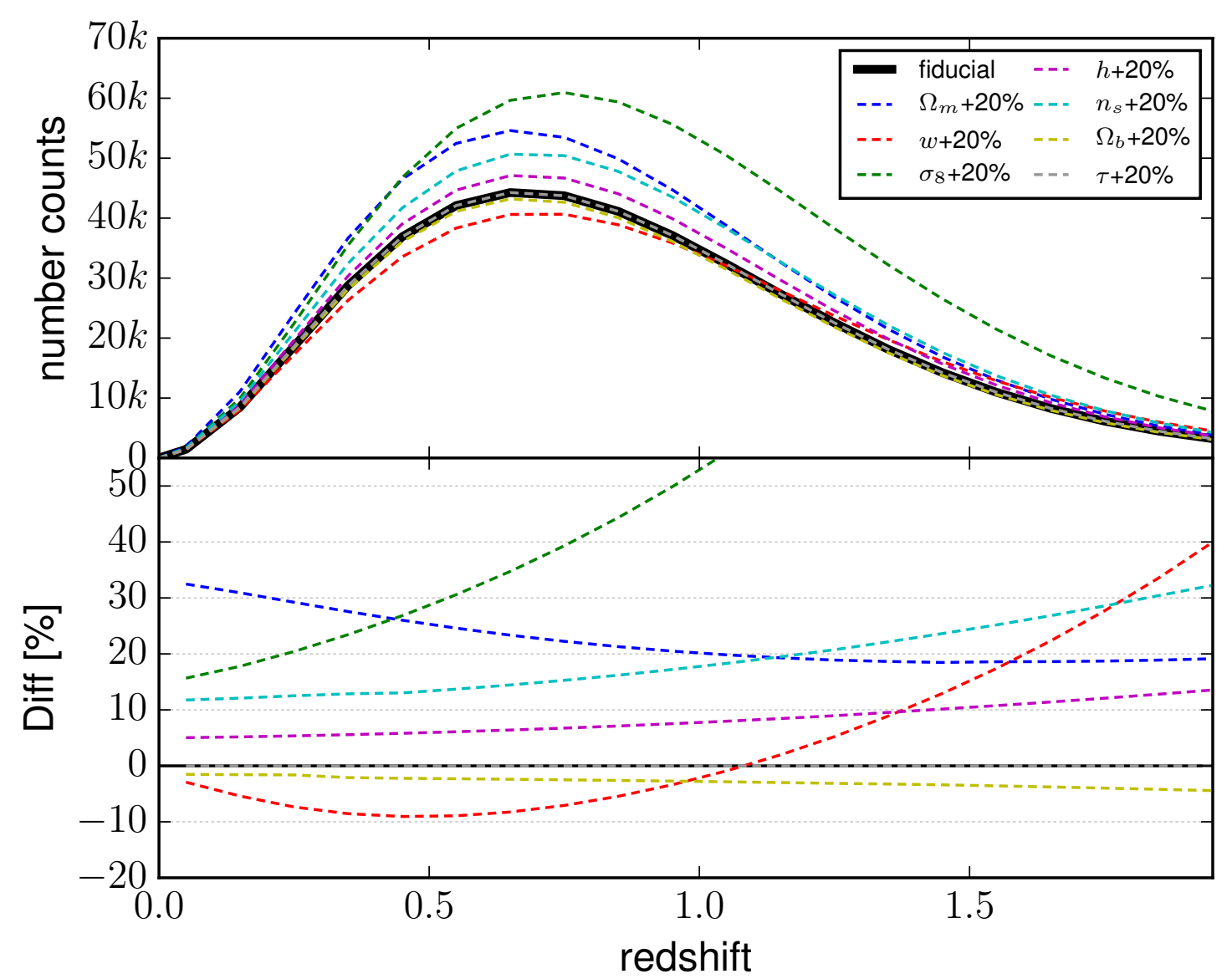

Figure 2.2: Halo number counts for $M>10^{13.8} M_{\odot} h^{-1}$ with fiducial cosmology defined in section 2.4 and a positive variation of $20 \%$ in each cosmological parameter keeping all the others fixed. On the bottom panel displays the relative variation relative to the fiducial case. The increase of $\Omega_{m}, \sigma_{8}, n_{s}$ and $h$ result in an increase of the abundance of halos, while an increment of $\Omega_{b}$ leads to a decrease on the halo abundance. The variation with $w$ is not monotonic, leading to a smaller abundance of halos at lower redshifts $(z \lesssim 1.1)$ and an increase at higher redshifts $(z \gtrsim 1.1)$.

\subsection{Halo Bias}

The theory calculations described above predict the mean abundance of halos observed in a certain volume and mass range. The actual number measured around each point in space $\vec{x}$ is a fluctuation around this average caused by the growth of perturbations on the dark matter density. However, halos are not a perfect tracer of dark matter. To understand this effect, one can consider the Peak-Background split (Bardeen et al., 1986, Cole \& Kaiser, 1989) in the 
fluctuations $\delta$ of the density field,

$$
\delta=\delta_{b}+\delta_{p}
$$

where $\delta_{b}$ is the background fluctuation, corresponding to the dark matter field perturbations and $\delta_{p}$ is an enhancement due to the peaks. The basic idea is that the presence of peaks effectively enhance the background, increasing the probability of halo formation. This is formally equivalent to lowering the actual threshold for the collapse and formation of halos to $\delta_{c p}=\delta_{c}-\delta_{b}$. This introduces a bias factor $b(z, M)$ in the observed halo number distribution compared to its average value. The effect of this Peak-Background split in the differential number density is given by

$$
\frac{d n(z, M, \vec{x})}{d \ln M}=\frac{d \bar{n}(z, M)}{d \ln M}[1+b(z, M) \delta(\vec{x})]
$$

In an analogy with Eq. 2.34, an average bias for the number density in a mass bin $\alpha$ can be defined as

$$
b_{\alpha}(z) \equiv \frac{1}{\bar{n}_{\alpha}} \int_{M_{\alpha}}^{M_{\alpha+1}} \frac{d M}{M} \frac{d \bar{n}}{d \ln M} b(z, M)
$$

and the local number density of halos can be generalized as

$$
n_{\alpha}(\vec{x}, z)=\bar{n}_{\alpha}\left[1+b_{\alpha}(z) \delta(\vec{x})\right]
$$

Within the Peak-Background split, the bias is fully determined by the functional form of the mass-function, as seen in the next examples. The results for the multiple mass functions presented are shown in Fig. 2.3, where we plot the halo bias as a function of halo mass $M$ at $z=0$ and their ratio to the Press-Schechter mass function.

\subsubsection{Press-Schechter Bias}

Using the Press Schechter (Press \& Schechter, 1974) differential comoving number density (Eq. 2.26) for the peak field $\left(\delta_{c} \rightarrow \delta_{c p}\right.$ ) and Taylor expanding around the background $\delta_{b}$ results 
in

$$
\begin{aligned}
\frac{d n_{p}}{d \ln M} & =\frac{d \bar{n}}{d \ln M}+\frac{\partial}{\partial \delta_{b}}\left(\frac{d \bar{n}}{d \ln M}\right) \delta_{b}+\ldots \\
& \approx \frac{d \bar{n}}{d \ln M}\left[1+\frac{v^{2}-1}{\delta_{c}} \delta_{b}\right]
\end{aligned}
$$

where $v=\delta_{c} / \sigma$. From Eq. 2.38 and Eq. 2.41, one could recover the bias function as $b(v)=$ $\left(v^{2}-1\right) / \delta_{c}$. However, as it has been discussed extensively (Kaiser, 1984; Cole \& Kaiser, 1989; Mo \& White, 1996), this approach for computing $d n_{p} / d \ln M$ is a calculation in Lagrangian space, resulting in the Lagrangian bias $b_{L}$. The bias $b$ in Eulerian space is related to $b_{L}$ via the transformation $b=1+b_{L}$ (Zel'dovich, 1970; Bardeen et al., 1986; Catelan et al., 1998)

$$
b(v)=1+\frac{v^{2}-1}{\delta_{c}} .
$$

\subsubsection{Sheth-Tormen Bias}

Based on the Sheth-Tormen mass function, this functional form (Sheth \& Tormen, 1999) can be obtained by following the same procedure of Press-Schechter bias, and has a significant correction on less massive halos. This provided a better agreement with simulations, where the parameters $a$ and $p$ were the same as in the Sheth-Tormen mass function. The resulting bias function is given by

$$
b(v)=1+\frac{a v^{2}-1}{\delta_{c}}+\frac{2 p}{\delta_{c}\left[1+\left(a_{c} v^{2}\right)^{p}\right]} .
$$

Again, we have that setting $a=1$ and $p=0$, the Press-Schechter results are recovered.

\subsubsection{Tinker Bias}

More recently, an empirical functional form for the bias was proposed by Tinker et al. (2010) as

$$
b(v)=1-A \frac{v^{a}}{v^{a}+\delta_{c}^{a}}+B v^{b}+C v^{c}
$$

This more flexible parametrization was shown to provide even better fits to simulation results. Similarly to the Tinker mass function, the free parameters $A, a, B, b, C, c$ depend on the value of 
the overdensity chosen $\Delta$ to detect spherical halos in the simulations.
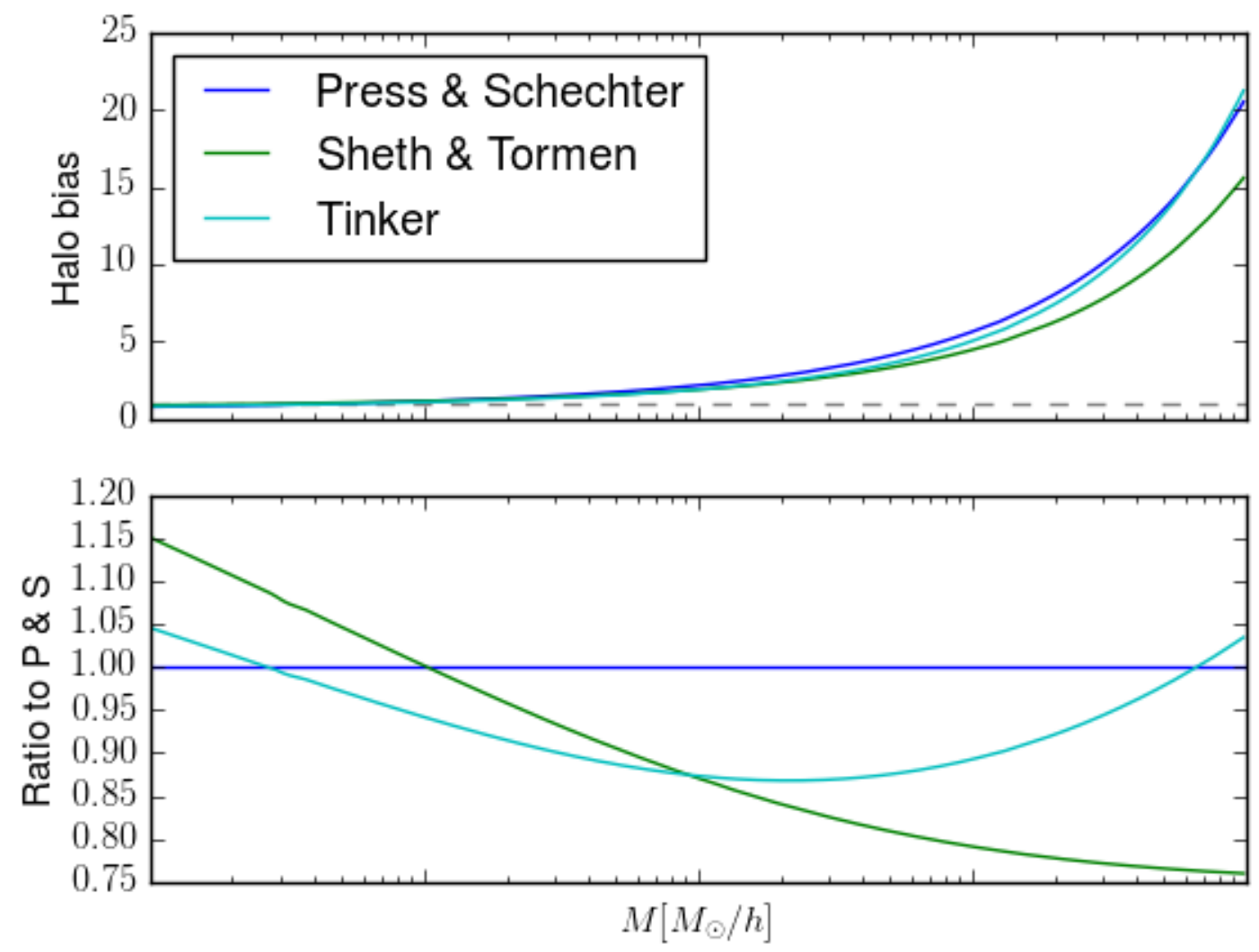

Figure 2.3: Halo bias as a function of halo mass at $z=0$, for the different mass functions in a $\Lambda \mathrm{CDM}$ model. The lower panel shows the ratios of the different mass functions relative to that of Press \& Schechter.

\subsection{Halo Covariance}

In previous sections, we considered theoretical predictions for the mean number of dark matter halos. The actual number of halos is subject to spatial dark matter fluctuations, such that halos are biased tracers of the dark matter density field. As a result, the halo counts have a covariance due to large scale structure, which can be calculated as

$$
\begin{aligned}
S_{i j}^{\alpha \beta} & =\left\langle\left(m_{\alpha, i}-\bar{m}_{\alpha, i}\right)\left(m_{\beta, j}-\bar{m}_{\beta, j}\right)\right\rangle_{i j} \\
& =\left\langle\int d V \bar{n}_{\alpha}(z) b_{\alpha}(z) \delta(\vec{x}) \int d V^{\prime} \bar{n}_{\beta}\left(z^{\prime}\right) b_{\beta}\left(z^{\prime}\right) \delta\left(\vec{x}^{\prime}\right)\right\rangle_{i j},
\end{aligned}
$$

where the brackets represent the statistical mean over several realizations of the redshift bins $i j$. 
We refer to this covariance due to large scale structure as sample variance (SV) or sample covariance. If the redshift bin $i$ is small enough, one can approximate $b_{\alpha}(z) \approx b_{\alpha, i}$ and $\bar{n}_{\alpha}(z) \approx$ $\bar{m}_{\alpha, i} / V_{i}$ and the sample covariance can be expressed as

$$
S_{i j}^{\alpha \beta}=\bar{m}_{\alpha, i} b_{\alpha, i} \bar{m}_{\beta, j} b_{\beta, j}\left\langle\int \frac{d V}{V} \delta(\vec{x}) \int \frac{d V^{\prime}}{V^{\prime}} \delta\left(\vec{x}^{\prime}\right)\right\rangle_{i j}
$$

In the equation above, it can be seen that the spatial dependence of the sample variance can be completely separated from the mass function. Therefore it is convenient to introduce the socalled spatial sample covariance $\left(S_{i j}\right)$, defined via $S_{i j}^{\alpha \beta} \equiv \bar{m}_{\alpha, i} b_{\alpha, i} \bar{m}_{\beta, j} b_{\beta, j} S_{i j}$. The quantity to be computed is the expected value of $\delta(\vec{x}) \delta\left(\vec{x}^{\prime}\right)$, given the window functions $W_{i}(\vec{x})$ and $W_{j}\left(\vec{x}^{\prime}\right)$ which delineate the corresponding volume elements

$$
\begin{aligned}
S_{i j} & =\int d W_{i} \int d W_{j}\left\langle\delta^{*}(\vec{x}) \delta\left(\vec{x}^{\prime}\right)\right\rangle \\
& =\int d W_{i} \int d W_{j} \int \frac{d^{3} k}{(2 \pi)^{3}} \int \frac{d^{3} k^{\prime}}{(2 \pi)^{3}} \exp [i \vec{k} \cdot \vec{x}] \exp \left[-i \vec{k}^{\prime} \cdot \vec{x}^{\prime}\right]\left\langle\delta(\vec{k}) \delta\left(\vec{k}^{\prime}\right)\right\rangle \\
& =\int d W_{i} \int d W_{j} \int \frac{d^{3} k}{(2 \pi)^{3}} \exp \left[-i \vec{k} \cdot\left(\vec{x}^{\prime}-\vec{x}\right)\right] P(k) \\
& =\int \frac{d^{3} k}{(2 \pi)^{3}} P(k) F_{i}^{*}(k) F_{j}(k),
\end{aligned}
$$

with the definitions $\int d W \equiv \int d V / V$ and $F_{i}(k) \equiv \int d W_{i} \exp [-i \vec{k} \cdot \vec{x}]$. This spatial sample covariance will only depend on the geometry of the window from the observations. In the next sections this covariance will be computed for different windows of interest.

\subsubsection{Full sky volume}

When we consider the volume within the full sky area up to a certain comoving distance $r_{i}$, the window computation can be simplified due to spherical symmetry. In this case the volume element is $d V=r^{2} d r d \cos \theta d \phi$, where $r$ is the comoving radius, $\theta$ the polar angle and $\phi$ the azimuthal angle. By aligning the azimuthal direction of the coordinate system with the direction 
of $\vec{k}$, the volume-averaged Fourier transform of the window function can be computed as:

$$
\begin{array}{r}
F_{i}(k)=\frac{1}{V} \int_{0}^{2 \pi} d \phi \int_{-1}^{1} d \cos \theta \int_{0}^{r_{i}} d r r^{2} \exp [-i k r \cos \theta]= \\
3 \frac{[\sin (k r)-(k r) \cos (k r)]_{0}^{r_{i}}}{\left.k^{3} r^{3}\right|_{0} ^{r_{i}}}=3 \frac{\left[(k r)^{3} j_{1}(k r)\right]_{0}^{r_{i}}}{\left.k^{3} r^{3}\right|_{0} ^{r_{i}}}=3 j_{1}\left(k r_{i}\right),
\end{array}
$$

where $j_{1}(x)=(\sin x-x \cos x) / x^{3}$ is the spherical Bessel function of first order. Hence, the sample variance between two full sky volumes of radius $r_{i}$ and $r_{j}$ can be computed as

$$
S_{i j}=\int_{-\infty}^{\infty} d \ln k 9 j_{1}\left(k r_{i}\right) j_{1}\left(k r_{j}\right) \frac{k^{3} P(k)}{2 \pi^{2}}
$$

\subsubsection{Full sky shell}

When the observation of cosmological surveys have redshifts precise enough to bin the data, the resulting windows are shells instead of the full sky volume. Hence, it is interesting to compute the covariance in a given shell with a defined thickness. Investigating the cluster covariance across the redshift shells, we can reconstitute the cosmological evolution of the large scale structure formation at different epochs.

Using spherical symmetry, the window function can be computed with Eq. 2.48 replacing the limits with a radius $r_{i}$ and the thickness of the shell $\delta r_{i}$ :

$$
F_{i}(k)=3 \frac{\left(k r_{i}+k \delta r_{i}\right)^{2} j_{1}\left(k r_{i}+k \delta r_{i}\right)-\left(k r_{i}\right)^{2} j_{1}\left(k r_{i}\right)}{k^{3}\left(\delta r_{i}^{3}+3 r_{i} \delta r_{i}^{2}+3 r_{i}^{2} \delta r_{i}\right)}
$$

If we take the approximation where the thickness of the shell is much smaller than its radial distance $\left(\delta r_{i} \ll r_{i}\right)$, the window function can be computed as

$$
F_{i}(k) \approx \frac{2}{k^{3} r_{i}^{2} \delta r_{i}} \sin \left(\frac{k \delta r_{i}}{2}\right)\left[\cos \left(k r_{i}\right)+\left(k r_{i}\right) \sin \left(k r_{i}\right)\right]
$$

\subsubsection{Cylindrical window}

For observations in a circular area with small radius $\left(\theta_{s} \lesssim 10 \mathrm{deg}\right)$ and a redshift bin $i$, the volume can be approximated by a cylinder with volume element $d V=\rho d \rho d \theta d r$, where $r$ is along the line of sight (i.e. the comoving radial distance), $\rho$ the radial component in cylindrical coordinates and $\theta$ the azimuthal angle. In this case the resulting window function is $(\mathrm{Hu} \&$ 
Kravtsov, 2003; Lima \& Hu, 2007)

$$
\begin{aligned}
& F_{i}(k)=\frac{1}{V} \int_{0}^{2 \pi} d \theta \int_{0}^{\theta_{s} r_{i}} d \rho \rho \int_{r_{i}-\delta r_{i} / 2}^{r_{i}+\delta r_{i} / 2} d r \exp \left[-i\left(k_{\perp} \rho \cos \theta+k_{\|} r\right)\right]= \\
& {\left[2 \exp \left[-i k_{\|} r_{i}\right] j_{0}\left(\frac{k_{\|} \delta r_{i}}{2}\right)\right] \frac{J_{1}\left(k_{\perp} \theta_{s} r_{i}\right)}{k_{\perp} \theta_{s} r_{i}} . }
\end{aligned}
$$

where $k$ was decomposed between the direction parallel to the line of sight $k_{\|}$and the perpendicular direction $k_{\perp}$ with $k \equiv \sqrt{{k_{\perp}}^{2}+k_{\|}^{2}}$. The resulting spatial sample covariance for a cylindrical window will be

$$
\begin{aligned}
S_{i j}= & \int_{-\infty}^{\infty} d \ln k_{\perp} k_{\perp}{ }^{2} 2 \frac{J_{1}\left(k_{\perp} \theta_{s} r_{i}\right)}{k_{\perp} \theta_{s} r_{i}} 2 \frac{J_{1}\left(k_{\perp} \theta_{s} r_{j}\right)}{k_{\perp} \theta_{s} r_{j}} \times \\
& \int_{-\infty}^{\infty} d \ln k_{\|} k_{\|} j_{0}\left(\frac{k_{\|} \delta r_{i}}{2}\right) j_{0}\left(\frac{k_{\|} \delta r_{j}}{2}\right) \cos \left(k_{\|}\left[r_{i}-r_{j}\right]\right) \frac{P(k)}{2 \pi^{2}} .
\end{aligned}
$$

\subsubsection{Cubical Window}

This approach is valid when the considered volume is a square with small area $(\delta x / r(z) \lesssim 10$ deg). It is useful when splitting the total survey in small boxes. In this case, the volume element is written in cartesian coordinates as $d V=d x d y d z$ and the window function becomes ( $\mathrm{Hu} \&$ Cohn, 2006)

$$
F_{i}(k)=\prod_{\chi=(x, y, z)}\left[2 \exp \left[-i k_{\chi} \chi_{i}\right] j_{0}\left(\frac{k_{\chi} \delta \chi_{i}}{2}\right)\right]
$$

Using the window function above, the spatial sample covariance is

$$
S_{i j}=\int_{0}^{\infty} \prod_{\chi=(x, y, z)} d k_{\chi} 2 \cos \left(k_{\chi}\left[\chi_{i}-\chi_{j}\right]\right) 2 j_{0}\left(\frac{k_{\chi} \delta \chi_{i}}{2}\right) 2 j_{0}\left(\frac{k_{\chi} \delta \chi_{j}}{2}\right) \frac{P(k)}{(2 \pi)^{3}}
$$

For a small squared area, this can be simplified with $\delta x_{i}=\delta y_{i}=r_{i} \delta \theta ; x_{i}=x_{j} ; \delta z=\delta r ; z_{i}=$ $r_{i}$ and the resulting spatial sample covariance is

$$
\begin{aligned}
S_{i j}= & \int_{0}^{\infty} d k_{z} 2 \cos \left(k_{z}\left[r_{i}-r_{j}\right]\right) 2 j_{0}\left(\frac{k_{z} \delta r_{i}}{2}\right) 2 j_{0}\left(\frac{k_{z} \delta r_{j}}{2}\right) \times \\
& \int_{0}^{\infty} 2 d k_{y} 2 j_{0}\left(\frac{k_{y} r_{i} \delta \theta}{2}\right) 2 j_{0}\left(\frac{k_{y} r_{j} \delta \theta}{2}\right) \times \\
& \int_{0}^{\infty} 2 d k_{x} 2 j_{0}\left(\frac{k_{x} r_{i} \delta \theta}{2}\right) 2 j_{0}\left(\frac{k_{x} r_{j} \delta \theta}{2}\right) \frac{P(k)}{(2 \pi)^{3}} .
\end{aligned}
$$




\subsubsection{Irregular windows}

In current and future large surveys, one of the goals is to maximize the area and depth of observations. However, even in full sky observations, contamination from sources such as stars and other celestial objects requires nontrivial masking of the observed area. As a result, the observed angular window function may present irregular shapes and holes. In these cases, analytical computations of the window function, as was done in the previous sections becomes nearly impossible. Moreover, computing the effects of such arbitrary window on the covariance may be be extremely expensive numerically. Recently we presented a method to compute the covariance of arbitrarily masked regions by decomposing the covariance in spherical harmonics (Lacasa et al., 2016) and using Fourier methods (Hamilton, 2000; Camacho et al., 2017) to efficiently perform the resulting integrals.

\subsubsection{Poisson Variance and Total Covariance}

The sample covariance (SV) computed so far is valid for large numbers of halos, where the fluctuations can be considered Gaussian. For a small numbers of halos, given either by a large mass threshold, high redshifts, or a small area, the distribution of halos may be dominated by Poisson noise (PN), with variance:

$$
P_{i j}=\bar{m}_{i} \delta_{i j}
$$

Lima \& Hu (2004) intoroduced the following form for computing the total covarince, that includes both approaches in the appropriate limits

$$
C_{i j}=S_{i j}+P_{i j}
$$

As can be seen Eqs. 2.45 and 2.57, the SV scales with $\bar{m}^{2}$, while the PN scales with $\bar{m}$. Consequently, PN has more significatvie contributions when the abundance of halos is small. In fig Fig. 2.4, we show the total covariance and each individual contribution, in a cylindrical window of $\Delta \Omega=10 \mathrm{deg}^{2}$ using the Tinker mass function for different mass thresholds. The total covariance is dominated by sample covariance when $\bar{m} \gtrsim 10$, and in regions where $\bar{m} \lesssim 10$, Poisson noise takes over. These limits are determined by a combination of both mass threshold and redshift, and SV dominates at lower thresholds and intermediate redshifts, around to the 


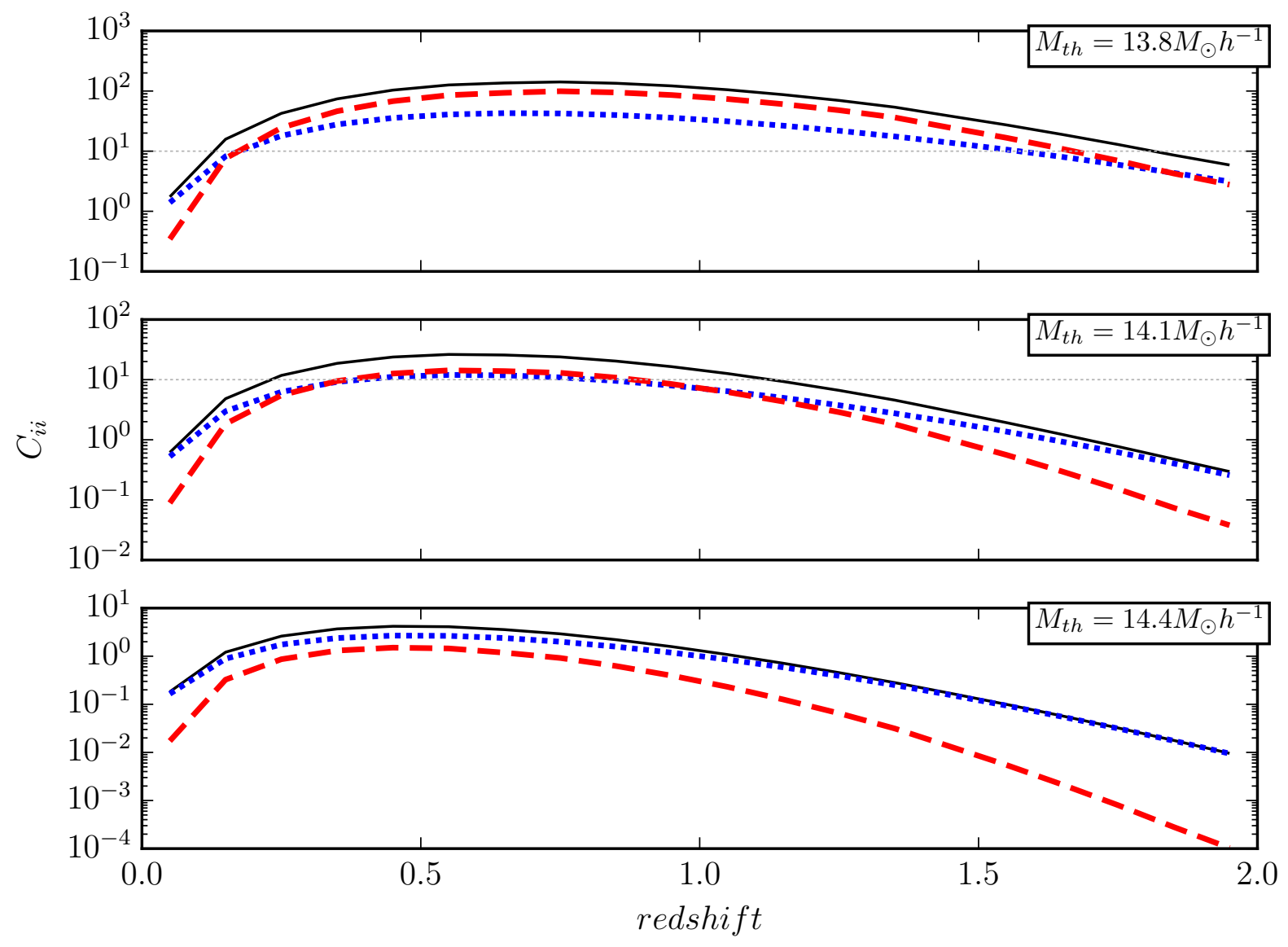

Figure 2.4: Diagonal of the cluster covariance, with the Tinker mass function in a cylindrical window of $10 \mathrm{deg}^{2}$ for different minimum mass thresholds. The total covariance (black solid line) is show along with the individual contributions from Poisson noise (blue dotted line) and Sample covariance (red dashed line).

peak of the halo number counts. 


\section{Chapter 3}

\section{Observations of Galaxy Clusters}

In order to constrain cosmological parameters using the abundance of galaxy clusters, several observational questions must be carefully examined. First, galaxy clusters are extended structures, and a clean detection of such objects may not be easily accomplished. Several techniques have been developed for detecting clusters at multiple wavelengths. Once clusters are detected, it is essential to determine their masses, as the cluster number counts are extremely sensitive e.g. to the mass threshold. Like many properties of astronomical bodies, the cluster mass is not a direct measurement, but an inferred quantity. As a result, if we intend to use clusters for cosmological purposes, the techniques for finding clusters must also provide a mass proxy, and the relation between true mass and the proxy must be established. Finally, even the best cluster finders are not perfect, and the selection effects associated to them must also be characterized. There might be e.g. false detections, missed clusters, projection effects and blending of objects. The lack of understanding of these effects could lead to significant offsets of inferred cosmological parameters.

In this chapter, we will discuss the possible techniques and algorithms for probing galaxy clusters. We will focus on the optical selection effects and describe the inclusion of these effects on theoretical predictions for cluster abundance. Our main interest will be on optically detected clusters, i.e. clusters detected by the spatial agglomeration of optical galaxies, and we highlight the WaZp code, used on later chapters of this work. We will also briefly present other methods for completeness purposes. The observational effects will be split into selection function related to the quality of the detection - and mass-proxy relation - describing the use of probes for the cluster mass determination. 


\subsection{Techniques for Detecting Clusters}

Due to the gargantuan size and internal structure of galaxy clusters, their identification is neither simple nor unique. One must distinguish e.g. between a real cluster and a substructure that appears angularly close, but that is not gravitationally bound. Several approaches including the inner physical processes of the clusters were developed to overcome this challenge in the detection process. The main techniques will be presented in this section.

\subsubsection{Optically Detected Clusters}

Probably the most straightforward method of cluster detection, finding optical clusters consists in seeking agglomerations of visible matter in optical galaxies. This technique enables the detection of clusters with information that can be easily observed, mainly angular positions and redshifts of galaxies. Though spectroscopic redshifts require extensive spectral information of each galaxy, photometric redshifts can be calculated based on the integrated fluxes observed in a few different bandpass filters. In the context of photometric surveys, it is possible to detect a larger number of galaxy clusters in wide areas of the sky. In this work, we will be primarily interested in optically detected clusters.

Most cluster finders reconstruct a density field from the catalog of galaxy positions, looking for clusters at peaks of density. Because this method relies on optical detections, it is more susceptible e.g. to galaxy redshift uncertainties as well as possible contaminations of galaxy samples by stars.

For optically detected clusters, the cluster richness can be used as a proxy for cluster mass. The richness is usually defined as the number of galaxies inside the cluster radius. Even though some cluster finders may adopt slightly different richness definitions, richness is always related to galaxy counts inside clusters. In order to obtain cluster masses, one can calibrate the massrichness relation in a sub-sample of clusters whose massses have been determined by other methods such as X-ray, SZ or lensing. Another approach is to self-calibrate the mass-richness relation along with the cosmology. The latter case leads to worse cosmological constraints, however, it is not subject to implicit assumptions underlying direct calibrations, which may bias cosmological results.

Using the cluster richness as a mass proxy generally requires an additional consideration of a non-negligible scatter on the mass-richness relation. The characterization of this scatter as 
well as a bias on the mass-richness relation can be as important as calibrating the relation itself (Lima \& Hu, 2005, 2007). A more in-depth explanation of the procedure for estimating the halo mass from the cluster richness is presented in section 3.3.2.

\subsubsection{Other Methods for Cluster Detection}

\section{X - ray Observations}

Most baryonic matter in the universe is found in the form of gas. Inside galaxy clusters, this gas composes the Intra-cluster medium (ICM) and is compressed by the strong gravitational field, causing it to heat to high temperatures of $\sim 10^{8} \mathrm{~K}$. At these large thermal energies, $\mathrm{X}$ ray radiation is emitted from the ICM mainly via Bremsstrahlung interaction, but also from recombination and bound-bound emission (Allen et al., 2011). Therefore, galaxy clusters are bright extended sources of X-ray.

The mass of a cluster is directly associated with the intensity of its X-ray emission. A rough prescription for the mass estimation can be described as follows: Assuming that the ICM is in equilibrium, one can relate the total mass of the cluster with the gas temperature. In a simple approximation, one can use the virial relation $E_{\text {kinetic }}+2 E_{\text {potencial }}=0$ and the fact that the velocity dispersion $\sigma^{2}$ is related to the temperature to estimate the relation between temperature $T$ of the ICM and cluster total mass

$$
T \propto \sigma^{2} \propto E_{\text {kinetic }}=-2 E_{\text {potential }} \propto \frac{M}{R}
$$

Supposing a constant density, $M \propto R^{3}$ and therefore $T \propto M^{2 / 3}$. Naturally, real applications of X-ray detections to estimate cluster masses are much more sophisticated and rigorous (Evrard et al., 1996; Vikhlinin et al., 2006; Clerc et al., 2016), but the assumptions are essentially the same. One of the main issues in this method is the measurement of the temperature distribution of the ICM. Additionally, these mass estimations are based on the hypothesis that the cluster is relaxed and in equilibrium. Since this is not true for many clusters (Sarazin, 1986), this assumption must be carefully taken into consideration as it may bias cluster mass estimates.

\section{Sunyaev-Zel'dovich Effect}

In 1969, Sunyaev and Zel'dovich predicted the Compton scattering of CMB photons, due to density perturbations in the Universe (Sunyaev \& Zeldovich, 1970). In the Sunyaev-Zel'dovich 
effect (SZE), CMB photons collide with high energy electrons and receive an energy boost through inverse Compton scattering. As a result, the frequency of CMB photons increase producing a shift of the CMB spectrum observed in that direction (Carlstrom et al., 2002). There are two different possibilities for galaxy clusters to produce the SZE. The main interaction of the CMB radiation occurs with high energy electrons of the ICM due to its intense heat; this leads to the so-called thermal SZE. Integrating along the line-of-sight through the gas of the cluster, it is possible to determinate the spectral distortion of the thermal SZE as the variation of temperature $\Delta T / T_{C M B}$. Within the same simplifications of the previous subsection, one could then relate the mass of the cluster to its temperature as $T \propto M^{2 / 3}$. However, as before, these measurements are dependent on the assumption of the cluster to be virialized.

The other, less stark, possibility of distortions on the CMB caused by clusters is the kinetic SZE. In this case, cluster peculiar velocities or bulk motions with respect to the CMB background add a Doppler shift, further distorting the CMB spectrum. A great advantage of SZ clusters, is that the thermal distortions on the CMB spectrum produced by a cluster are roughly independent of redshift (apart from angular resolution of the survey and angular sizes of clusters, which do depend on redshift). Consequently, high-redshift clusters can be detected via this method.

\section{Cluster Lensing}

Cluster Lensing is a technique for making precise measurements of cluster masses, though it is possible to detect clusters through shear-peak maps (Dietrich \& Hartlap, 2010; Marian et al., 2012; Kacprzak et al., 2016; Peel et al., 2017). Because Lensing is based on the deviation of light rays due to space-time distortions induced by gravitational potentials, it allows us to directly estimate the total cluster masses - both baryonic and dark matter - and does not require modeling complex astrophysical processes inside clusters.

Within General Relativity, matter deforms space-time, bending the light paths near massive objects. Such is the case for galaxy clusters in the line-of-sight of source galaxies. The cluster potentials produce a shear field around clusters, such that the deviation of light rays distorts the observed images of background galaxies. These distortions can be combined statistically to reconstruct the cluster gravitational potential and therefore its total mass (Schneider et al. 1992; Bartelmann \& Schneider, 2001).

The angular deviation of light caused by a gravitational lens can be characterized by the 
convergence field $\kappa$, defined from the projected surface density of the lens $\Sigma(\vec{\xi})$ and a critical surface density $\Sigma_{c r}$

$$
\kappa=\frac{\Sigma(\vec{\xi})}{\Sigma_{c r}} \quad ; \quad \Sigma_{c r} \equiv \frac{c^{2}}{4 \pi G} \frac{D_{S}}{D_{L} D_{S L}}
$$

where $\vec{\xi}$ is a $2 \mathrm{D}$ distance vector on the lens plane (perpendicular to the line-of-sight), $D_{S}$ and $D_{L}$ are the angular diameter distances from the observer to the source and to the lens respectively, and $D_{L S}$ is the angular diameter distance between the lens and the source. When $\kappa$ is larger, the lensing effect is intensified. This can result from having massive lenses, or from a lens-source configuration that maximizes the lensing efficiency.

There are two different approaches on gravitational lensing depending on the strength of the effect. In the panorama of galaxy clusters, Strong Lensing occurs when $\Sigma$ is large enough, such that arcs and/or multiple images of the source are produced. The use of several source images allows us to better reconstruct the galaxy source position and accurately estimate the lens potential or cluster mass.

The other limit corresponds to Weak Lensing, where galaxy images are only slightly changed and these distortions must be combined statistically. One may e.g. stack the shear fields from clusters of similar richnesses and estimate the average surface density of these clusters. Once the projected density is reconstructed, the average mass $M$ of these clusters is given by integrating the density over the cluster projected area

$$
M=\int d^{2} \xi \Sigma(\vec{\xi})
$$

\subsection{Halo and Cluster Finders}

The study of both dark matter halos and galaxy clusters introduces the initial challenge of detecting these structures. Due to the spatial extension of these objects and the fact that they are composed by a number of sub-structures such as sub-halos and galaxies, we must define their boundaries in addition to locating them. With these goals, several codes of object identification have been developed. We will refer to these generally as group finders, and they will be divided into halo finders - used to detect dark matter halos in N-body simulations - and cluster finders - designed to detect galaxy clusters.

These algorithms for object identification must not only detect halos and clusters, but also 
define their characteristics such as the size and number of members. Usually, the procedure is to find the candidate particles or regions where the centers of these groups can be located and then investigate the surroundings to qualify these candidates as a group and its features. The two main approaches on how to define the properties of groups are the Friends-of-Friend (FoF) (Huchra \& Geller, 1982; Botzler et al., 2004; Farrens et al., 2011) and Spherical-Overdensity (SO) (Lacey \& Cole, 1994; Planelles \& Quilis, 2010). These techniques are more applicable to halo finders as they are used only in simulations (Springel, 2005; Few et al., 2012; BoylanKolchin et al., 2009; Fosalba et al., 2015b; Crocce et al., 2015; Fosalba et al., 2015a), where the real positions of the objects are know. Most cluster finders have a more complex structure to take into account a diversity of observational effects, such as the quality of the observed redshift and blending in the line of sight. However, it is still possible to identify a resemblance to either FoF or SO at the core of the cluster finders algorithms.

Each definition of group finder has its advantages and limitations, and their application to the same galaxy catalog produces groups with different properties. As there is no definitive criterion for defining most characteristics of halos and clusters, such as size, limiting overdensity, shape, etc, there is no "correct" group finder. The choice of group finder is somewhat arbitrary and the important consideration is to guarantee consistency throughout the analysis. Thus we must apply the same approach to detect halos in simulations - from which we construct or calibrate theoretical predictions - and to find real clusters.

In Friends-of-Friend (FoF) algorithms, two particles are considered linked if the distance between them is less than the linking length, a parameter to be specified on each code. This linking process continues until there are no more particles within the range of the linked group. As a result, the members of $\mathrm{FoF}$ groups are unique and each particle belongs to only one group. Differently from SO algorithms, the architecture of FoF groups is not restricted to any specific shapes and the substructures are entirely located inside a host group. As the groups are detected based on the arbitrary choice of a linking length parameter, its value must be carefully adjusted, as a length that is too short will break or fragment coherent structures and a length that is too long will cause incoherent structures to over-merge.

Spherical-Overdensity (SO) algorithms focus on finding regions of large overdensity as potential group centers. Then the algorithm grows a sphere radius around the largest overdensity until the density within the sphere is $\Delta$ times the background density. The process is repeated for the next largest overdensity, until all groups have been found. Because halos/clusters are 
denser close to their centers, larger values of $\Delta$ lead to smaller radii. By definition, groups detected are defined within spheres, however, different groups may have intersections, in which a given particle/galaxy may belong to more than one group.

One nice feature of the SO method is the fact that SO groups are already defined with a characteristic size. This benefit is so advantageous that some group finders use FoF to detect groups centers, but then use a SO algorithm to compute their sizes. A typical, but arbitrary, value used for the overdensity is $\Delta=200$ (related to the background matter density $\bar{\rho}_{m}$ ) - defining a group radius $R_{200}$ - which is inspired by the virial overdensity ( $\Delta_{\mathrm{vir}} \approx 178$ ) within the spherical collapse model for an Einstein-de Sitter universe (see Eq. 2.19].

\subsubsection{Optical Cluster Finders: redMaPPer, VT and WaZp}

As discussed in previous sections, galaxy clusters can be detected with multiple techniques, based on different wavelengths of the light spectrum. Even within a certain type of observation, such as optical clusters, there are different methods or algorithms to locate galaxy clusters. We will focus on optical cluster finders, which generally have lower observational requirements compared to X-ray and SZ clusters. For instance, the detection of optical clusters does not require spectroscopic or long-period observations in a certain sky region. Instead, photometric wide-area surveys with observations on a few optical bands are usually enough. Consequently, it is typically possible to detect larger numbers of galaxy clusters in the optical spectral range. In this section, we will discuss the main cluster finders present in the DES, with emphasis on WaZp, as this is a code that has been developed and tested in direct collaboration with the DES-Brazil group.

The red-sequence Matched-filter Probabilistic Percolation cluster finder (redMaPPer) (Rykoff et al., 2014) is based on the well-known presence of so-called red-sequence galaxies within lowredshift galaxy clusters. The code starts from a red-sequence model and calibrates the redshift for all galaxies. Galaxies with reasonable fit to the red-sequence template are used as candidates for cluster central galaxies. For each candidate, the probability of being a central galaxy is estimated by constructing a cluster around the candidate and computing the cluster richness. Finally, sorting galaxies by these probabilities, the clusters are constructed and membership and richnesses are assigned. The clusters are then used to recalibrate the red-sequence model that will be used to compute new redshifts for the red-sequence galaxies and find new clusters. This process is iterated until a convergence is reached. As a result, this method could 
be expected to provide reliable calibrations even from rough initial conditions. The cluster members are defined based on the probability function for a galaxy to belong to a cluster, constructed using galaxy colors as well as their angular distances to the cluster center, while the richness is given by the sum of probabilities of galaxies closer than a given radius (usually $R_{200}$ or $\left.R_{500}\right)$. This method is efficient for the detection of clusters at low to intermediate redshifts, where red-sequence galaxies are easily located and their properties have been widely studied (Bell et al., 2004; Gladders \& Yee, 2005; Arnouts et al., 2007). However, for higher redshifts $(z>0.7)$, there is no guarantee that the red-sequence is dominant or easily seen in clusters, and this method may not produce reliable results. This cluster finder has been actively used with continuous improvements and studies of its properties (Rykoff et al. 2014, Rozo \& Rykoff, 2014, Rozo et al., 2015a b), including the estimation of cluster masses (Saro et al., 2015; Baxter et al., 2016; Simet et al., 2017), and it will soon be used for cosmological constraints within the DES.

The Voronoi Tesselation cluster finder (VT) (Soares-Santos et al., 2011) is based on galaxy over-densities in redshift layers. In this method, the space is sliced in several photometric redshift layers and each layer is fragmented with a Voronoi Tessellation. This process creates polygon cells based on the distance of each neighbor galaxy. As a result, each cell contains one galaxy, with cell area directly related to the local density. Then, the highest density regions are considered candidates to form clusters, and adjacent cells can be added according to their densities. In the end, all galaxies belong to a group, and clusters are defined by applying a richness cut. This process is similar to FoF and also results in clusters that are not necessarily spherical. Even though this cluster finder presents an innovative approach to the detection of optical clusters, it is still on a premature development stage concerning observational effects (Saro et al. 2015).

The Wavelet z Photometric (WaZp) cluster finder (Dietrich et al., 2014) has been developed by Christophe Benoist (Observatoire de la Côte d'Azur), and is being used and improved, in collaboration with the DES-Brazil team. For this cluster finder, space is also sliced in photometric redshift layers, but including an overlap between layers as shown in the left panel of Fig. 3.2.1. Then, in each slice, the surface density field is calculated based on the galaxy distribution, via a wavelet transform method. In this transformation, the galaxy brightness is discretized into pixels with size determined by a selected scale. As the same cluster may be located in more than one slice, the peaks of consecutive slices must be considered, and cylinders are constructed with 


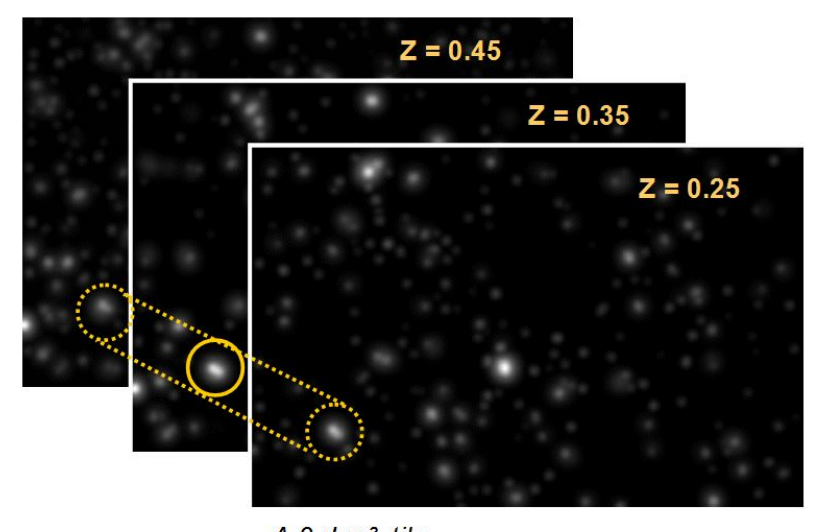

A $3 \mathrm{deg}^{2}$ tile



Figure 3.1: Snapshot of the procedures in the WaZp code. Left: An overdensity across redshift slices. Right: Membership assignment schematics.

radius based on the size of the overdensity. Then, the density across the redshifts of cylinders is computed, and the position of the cluster in redshift and angle is refined. The membership assignment (right panel of Fig. 3.2.1) is done based on probability using the angular distance of the galaxies to the cluster center, the local background and the photometric redshift information (Castignani \& Benoist, 2016). An advantage of locating clusters using spatial distribution only, is that it does not depend on an assumption of astrophysical nature such as the brightest cluster galaxy $(\mathrm{BCG})$ or the presence of a red-sequence.

This code was designed to be used on optical data, creating visibility maps, computing the local background to avoid false detections and using magnitude and redshift information to slice the data. Results from the WaZp code in a simulation comparing galaxy clusters to dark matter halos, including measured observational effects are shown in section 6.2.3.

\subsection{Observational Effects of Galaxy Clusters}

In chapter 2, a theoretical framework for predicting the abundance of dark matter halos was presented. However, real optical observations can only detect the galaxies that trace the density of halos. As the gravitational field of dark matter halos leads to baryonic agglomeration driving the formation of collapsed structures, we should expect galaxy clusters to be tightly related to the underlying halos. With these considerations, there are two main issues on using clusters as tracers for halos.

Firstly, the properties that can be measured on clusters are not necessarily the same as the halo properties. While the halo formalism was based on the mass of halos, for clusters, in 
most cases, we only have a mass proxy. Additionally, if the clusters are detected in a photometric survey, the resulting cluster photometric redshifts are not as reliable as spectroscopic redshifts. In those cases, it is necessary an additional treatment relating photometric redshifts to "real" redshifts, i.e. photo-z errors. Studies of photometric redshifts are extremely important for cosmological work based directly on galaxy measurements of matter tracers, such as galaxy two-point correlation functions (Sobreira et al., 2011; de Simoni et al., 2013), quasar power spectra (Abramo et al., 2012), weak lensing (Ma et al., 2006) and even cluster properties (Lima $\& \mathrm{Hu}, 2007)$. However, as clusters are formed by many galaxies, we expect cluster redshifts to be more precise than photo-zs of individual galaxies, and not an important issue for cluster cosmological constraints (Lima \& Hu, 2007). As a consequence, this specific photometric redshift issue for clusters is usually not considered. Nonetheless, we will present a brief framework to include such effects on cluster predictions.

The second issue is related to a selection function, concerning the correspondence between dark matter halos and galaxy clusters. Using clusters as tracers of dark matter halos seems to require the assumption that there is a corresponding halo to each cluster and vice-versa. Even if we consider that there is a one to one equivalence, as the presence of dark matter halos causes galaxy cluster formation, we may still have an incorrect correspondence, introduced by the imperfect performance of algorithms used for galaxy cluster detection. Cluster finders can fail to locate a fraction of the clusters associated to dark matter halo, as well as produce false detections. These two imperfections are characterized by the completeness and purity of cluster samples, which mainly reflect the limitations of the cluster finder.

In the next sections, we will present considerations for each of these observational effects and how to model them, culminating in a realistic theoretical prediction for galaxy cluster abundance.

\subsubsection{Photometric Redshifts}

Currently, there are several photometric surveys in progress such as SDSS (York et al., 2000), CFHTLS (Pierre et al., 2004) and DES (Flaugher, 2005) and a few others being planned such as LSST (Tyson, 2002) and Euclid (Amendola et al., 2013). The main goals of all these surveys is to use multiple optical observables to constrain cosmological models. Photometric observations can cover considerably wider areas on the sky with lower costs and less time than spectroscopic surveys, resulting in hundreds of millions of objects observed. Using observations in a few 
different filters, it is even possible to compute the redshift of objects, known as photometric redshifts (photo-zs). However, the quality of these photo-zs is considerably lower than "real" spectroscopic redshifts (spec-zs), and theoretical predictions must account for the use of photozs.

Photometric redshifts are computed by photo-z codes (Arnouts \& Ilbert, 2011; Carrasco Kind \& Brunner, 2013, 2014; Benítez, 2000), based on magnitudes of the objects in different filters. As a result, photo-z errors are mainly tied to degeneracies in color-magnitude-redshift space and the efficiency of the photo-z algorithms (Oyaizu et al., 2008; Abdalla et al., 2011; Sánchez et al., 2014). The relation between photo-zs and real redshifts can be characterized by a probability distribution $P\left(z^{\text {phot }} \mid z\right)$ of having a certain value of photo-z $\left(z^{\text {phot }}\right)$ given the redshift. This distribution is usually well described by a Gaussian distribution (Lima \& Hu, 2007),

$$
P\left(z^{\text {phot }} \mid z\right)=\frac{1}{\sqrt{2 \pi \sigma_{z^{\text {phot }}}^{2}}} \exp \left[-\frac{\left(z^{\text {phot }}-z-z_{\text {bias }}^{\text {phot }}\right)^{2}}{2 \sigma_{z^{\text {phot }}}^{2}}\right]
$$

where the scatter $\sigma_{z^{\text {phot }}}(z)$ and bias $z_{\text {bias }}^{\text {phot }}(z)$ can be a function of the real redshift $z$. Predictions of quantities $\mathcal{P}\left(z^{\text {phot }}\right)$ as a function of photo-zs can be computed by integrating the original prediction $\mathcal{P}(z)$ over all redshifts, weighted by $P\left(z^{\text {phot }} \mid z\right)$ :

$$
\mathcal{P}\left(z^{\text {phot }}\right)=\int_{0}^{\infty} d z \mathcal{P}(z) P\left(z^{\text {phot }} \mid z\right)
$$

We note that the redshift of clusters in photometric surveys are much more precise and accurate than photo-zs for individual galaxies, as the cluster redshift can be estimated by its multiple galaxy member photo-zs. Hence, errors on galaxy clusters redshifts is typically assumed to be negligible (Lima \& Hu, 2007).

\subsubsection{Mass-Observable Relation}

In order to use clusters for cosmology, we must estimate the masses of the dark matter halos associated with galaxy clusters. Unfortunately, the mass is not a direct observable and must be inferred. There are several methods to estimate the mass of galaxy clusters, including those presented in the last section for cluster detection, such as weak lensing, Sunyaev-Zel'dovich effect, X-rays and also galaxy velocity dispersion and stellar light. These techniques infer the 
mass via a proxy, for X-rays it is the temperature of the interstellar cluster gas, for SZ it is the integrated Compton parameter and for optically detected clusters it is the cluster richness. In an ideal scenario, the mass proxy would have a perfect relation with halo mass. This relation, however, is characterized by a distribution with a mean relation and a non negligible scatter (Levine et al., 2002, Lima \& Hu, 2004; Majumdar \& Mohr, 2004, Lima \& Hu, 2005).

We can characterize the mass-proxy distribution via $P(Y \mid M)$, the probability of having the mass proxy $Y$, given the true mass $M$. Integrating $P(Y \mid M)$ with the mass function $d \bar{n} / d M$, we can compute a proxy function $d \bar{n} / d Y$ that describes the cluster distribution as a function of the proxy. It is also possible to go on the other direction and obtain $P(M \mid Y)$, the probability of having the true mass of the cluster $M$, given the proxy $Y$. The relation between $M$ and $Y$ can be understood statistically by considering the mass function as the probability $P(M)$ of having a halo with mass between $M$ and $M+d M$ and the proxy function as the probability $P(Y)$ of having a cluster with proxy between $Y$ and $Y+d Y$. Using Bayes Theorem $P(M \mid Y) P(Y)=$ $P(Y \mid M) P(M)$, we have

$$
P(M \mid Y) d M \frac{d \bar{n}}{d Y} d Y=P(Y \mid M) d Y \frac{d \bar{n}}{d M} d M
$$

Because the mass range covers several orders of magnitude, it can be more convenient to consider the distributions in log space:

$$
P(\ln M \mid \ln Y) d \ln M \frac{d \bar{n}}{d \ln Y} d \ln Y=P(\ln Y \mid \ln M) d \ln Y \frac{d \bar{n}}{d \ln M} d \ln M
$$

This equation describes the probability of having a cluster of both mass $M$ and proxy $Y$. For notation simplification, from this point on, we will represent the probabilities in log space by: $P(\ln M \mid \ln Y) \rightarrow P(M \mid Y) ; P(\ln Y \mid \ln M) \rightarrow P(Y \mid M)$. All equations apart from 3.6 and 3.7 will follow this new notation.

In order to have the probability of having a cluster with proxy $\ln Y$, i.e. the proxy function, we integrate Eq. 3.7 on both sides over all possible masses (and use the normalization $\left.\int_{-\infty}^{\infty} d \ln M P(M \mid Y)=1\right)$ :

$$
\frac{d \bar{n}}{d \ln Y}=\int_{-\infty}^{\infty} d \ln M \frac{d \bar{n}}{d \ln M} P(Y \mid M)
$$

Now the abundance of clusters can be predicted in a bin $\alpha$ of the proxy by replacing Eq. 2.34 
with

$$
\bar{n}_{\alpha}(z) \equiv \int_{\ln Y_{\alpha}}^{\ln Y_{\alpha+1}} d \ln Y \frac{d \bar{n}}{d \ln Y}=\int_{\ln Y_{\alpha}}^{\ln Y_{\alpha+1}} d \ln Y \int_{-\infty}^{\infty} d \ln M \frac{d \bar{n}}{d \ln M} P(Y \mid M)
$$

It is important to point that the results in this section were derived considering there is a perfect correspondence between halos and clusters. That is, there is always a dark matter halo associated with each cluster detected. If this assumption is violated, the equations must be corrected accordingly. In the next section we will explore these cases.

In a basic approach, one might expect the mass proxy to scale with the real mass, resulting in a power-law relation. If the scatter in such relation occurs mostly due to fluctuations from the proposed model of estimating the mass, the distribution of the mass proxy can be modeled as a Gaussian in logarithm space

$$
P(Y \mid M)=\frac{1}{\sqrt{2 \pi \sigma_{\ln Y}^{2}(M)}} \exp \left[\frac{(\ln Y-\langle\ln Y \mid \ln M\rangle)^{2}}{2 \sigma_{\ln Y}^{2}(M)}\right]
$$

where $\langle\ln Y \mid \ln M\rangle$ is the mean proxy-mass relation and $\sigma_{\ln Y}(M)$ is the scatter in the relation. This scatter might have different values for different masses/proxies, but one could expect that it should decrease at large masses, where the mass-proxy is typically more well-defined and better calibrated (at least for values of proxy and mass in which clusters can still be found). Additionally, the distribution might not be Gaussian for both the mass at fixed proxy and for the proxy at fixed mass and this assumption should be checked. Simulations indicate that the intrinsic mass-proxy relation for some observables is indeed well described by a log-normal distribution (Nagai, 2006; Kravtsov et al., 2006).

Using these relations, this work will make cosmological constraints based on the proposition from (Lima \& Hu, 2005) to self-calibrate the mass of clusters with respect to their richnesses. This approach consists of constraining the mass-richness parameters along with the cosmology. External cross-calibrations - from other proxies or from simulations - of this relation can easily be included in the formalism developed as priors in the mass-richness relation, which, when available, can improve cosmological constraints from optical clusters.

In section 5.1, we will study specifically the difference between $P(Y \mid M)$ and $P(M \mid Y)$, how they are related, and the resulting $P(M \mid Y)$ from a Gaussian $P(Y \mid M)$ and assuming a Tinker (Tinker et al., 2008) mass function. 


\subsubsection{Completeness and Purity}

Several factors can induce a non-perfect detection of galaxy clusters. From intrinsic causes, such as the probabilistic nature of models for structure formation in the universe and consequently the fact that clusters do not follow theoretical predictions individually but only statistically, to projection effects and even limitations of the cluster finder algorithms. Likewise, it is not possible to perfectly predict the abundance of galaxy clusters using the formalism as stated in chapter 2. In order to use the abundance of cluster for cosmological purposes, the effects related to imperfect detections must be included statistically.

The completeness is the fraction of galaxy clusters found associated with dark matter halos relative to the total number of halos. The range of values for completeness goes from 0 to 1 , where 0 indicates no objects were found and 1 reflects that all halos were correctly detected as clusters, resulting in a complete catalog. The purity is the fraction of clusters found associated with halos relative to the total number of clusters found, with values also between 0 and 1 . If the purity is less than 1, this means that either some objects detected by the cluster finder may not be real galaxy clusters or that some halos were split into multiple clusters.

As the effects of completeness and purity directly inflict on the observed abundance of galaxy clusters, it is necessary to account for them in the connection between observed clusters and theoretical halos. These considerations lead to additional complications on the theoretical prediction for cluster counts. Currently, most works limit the analyses of clusters to higher masses and richnesses, where both completeness and purity are close to 1 , and can therefore be neglected (Rykoff et al., 2014; Rozo \& Rykoff, 2014). However, in this section we will lay out a framework to include those effects, allowing us to investigate halos/clusters with lower masses/richnesses.

The probability of detecting a "true" cluster of mass proxy $Y$ is the probability of having a cluster, "filtered" by the purity $p(Y)$. In the same manner, the probability of having halos associated to those clusters is the probability of having a dark matter halo convoluted with the completeness $c(M)$. Bayes' Theorem applied to these objects allows us to write then

$$
P(M \mid Y) d M \frac{d \bar{n}}{d \ln Y} p(Y) d \ln Y=P(Y \mid M) d \ln Y \frac{d \bar{n}}{d \ln M} c(M) d \ln M
$$


and the number density of detected clusters with mass proxy in the range $\left[\ln Y_{\alpha}, \ln Y_{\alpha+1}\right]$ is

$$
\bar{n}_{\alpha}(z)=\int_{\ln Y_{\alpha}}^{\ln Y_{\alpha+1}} d \ln Y \int_{-\infty}^{\infty} d \ln M \frac{d \bar{n}}{d \ln M} P(Y \mid M) \frac{c(M)}{p(Y)}
$$

As clusters of high masses stand out in observations, we expect less ambiguity in detecting them. Therefore the completeness and purity should approach unity at high enough value of $M$ (and $Y$ ). Similarly, for low masses, the number of clusters increase and we expect the confusion to be larger, so the completeness and purity decrease. Thus, we propose functional forms for both completeness and purity that follow these mass limits

$$
\begin{aligned}
c(M, z) & =\frac{\left[M / M_{c}(z)\right]^{n_{c}}}{\left[M / M_{c}(z)\right]^{n_{c}}+1}, \\
p\left(M^{\mathrm{obs}}, z^{\text {obs }}\right) & =\frac{\left[M^{\mathrm{obs}} / M_{p}^{\mathrm{obs}}\left(z^{\mathrm{phot}}\right)\right]^{n_{p}}}{\left[M^{\mathrm{obs}} / M_{p}^{\text {obs }}\left(z^{\mathrm{phot}}\right)\right]^{n_{p}}+1}
\end{aligned}
$$

where $M_{c}(z)$ and $M_{p}(z)$ are parametrized functions and we take the exponents $n_{c}$ and $n_{p}$ to be constant. The completeness will be taken as a function of mass $M$ and redshift $z$ of the real dark matter halos, while the purity will depend on cluster observed quantities, i.e. photo-z or $z^{o b s}$, and the mass proxy $Y$ or observed mass $M^{\text {obs }}$.

For the mass scale $M_{c}$ and proxy scale $M_{p}$ which control the transition in the selection function, we will take a very simple approach, with linear relations:

$$
\begin{aligned}
\log M_{c}(z) & =\log M_{c f i d}+c_{0}+c_{1}(1+z) \\
\log M_{p}^{\mathrm{obs}}(z) & =\log M_{p f i d}^{\mathrm{obs}}+p_{0}+p_{1}(1+z)
\end{aligned}
$$

As it will be seen in section 6.2 .3 , this functional form describes the completeness and purity measured in the simulation.

\subsubsection{Theoretical Prediction for the Abundance of Observed Clusters}

The parameters used to characterize the measurement of galaxy clusters $\left(\Theta_{c l}\right)$ are not the same parameters that describe the dark matter halos $\left(\Theta_{h}\right)$. In order to make a prediction for the abundance of clusters it is necessary a window function $W_{[c l, h]}$ that relates a volume in the $\Theta_{c l}$ 
space to a volume in $\Theta_{h}$ space

$$
\frac{d \bar{n}}{d \ln X}\left(\Theta_{c l}\right)=\int d V_{\Theta_{h}} \frac{d \bar{n}}{d \ln M}\left(\Theta_{h}\right) W_{[c l, h]}
$$

where $X$ is the mass proxy used for the clusters.

As discussed in the previous sections, our approach to photometric surveys is to consider four components to the window function: A relation between photo-z and real redshift (section 3.3.1), a similar, albeit more complex, mass-proxy relation (section 3.3.2), and the sample selection function, i. e. completeness and purity (section 3.3.3). An important consideration is that there may be an interplay between the mass-proxy relation and the sample selection function, as the characterization of both depend on the matching process of clusters and halos (in simulations) and clusters and clusters (in multi-wavelength observations). For instance, clusters scattered in and out of a given proxy bin may affect the sample completeness and purity, an effect which may be parametrized by altering the mass-proxy relation to include an extra Gaussian term (Erickson et al., 2011). Conversely, using only clusters and/or halos which are believed to have been correctly matched to define the observable-mass relation may produce a relation with unrealistically low (and Gaussian) scatter. Despite these issues, it is conceptually simpler to keep the definitions of completeness and purity decoupled from the observable-mass relation, and we will follow this approach, parametrizing all these functions independently. With these considerations in mind, the complete window function can be described as

$$
W_{[c l, h]}=P\left(z \mid z^{\mathrm{phot}}\right) P\left(M \mid M^{\mathrm{obs}}\right) \frac{c(M, z)}{p\left(M^{\mathrm{obs}}, z^{\mathrm{phot}}\right)} .
$$

and the theoretical prediction for the abundance of clusters in a mass-proxy bin $(\alpha)$ and cluster photo-z bin (i) can finally be computed as

$$
\begin{aligned}
& \bar{m}_{\alpha, i} \quad \equiv \Delta \Omega \int_{z_{i}^{\text {phot }}}^{z_{i+1}^{\text {phot }}} d z^{\text {phot }} \int d z \frac{D_{A}(z)^{2}}{H(z)} P\left(z^{\text {phot }} \mid z\right) \\
& \int_{\ln M_{\alpha}^{\mathrm{obs}}}^{\ln M_{\alpha+1}^{\mathrm{obs}}} d \ln M^{\mathrm{obs}} \int d \ln M \frac{d \bar{n}}{d \ln M} P\left(M^{\mathrm{obs}} \mid M\right) \frac{c(M, z)}{p\left(M^{\mathrm{obs}}, z^{\mathrm{phot}}\right)}
\end{aligned}
$$




\section{Chapter 4}

\section{Statistical Methods}

Modern cosmological experiments aim to detect large numbers of objects and analyze them through advanced statistical methods in order to make reliable inferences. One important quantity in this context is the posterior, which defines the probability of a sample of parameters for a given measurement. Given the likelihood, one may use a more theoretical and faster approach - the Fisher Matrix (FM) technique - to forecast constraints of a certain probe in a survey. In a more realistic analysis, the likelihood can be used on Monte Carlo Markov Chain (MCMC) methods to constrain model parameters, requiring a considerably higher computational cost. Another important quantity is the sample covariance, directly connected to the quality of the constraints from dark matter halos. In section 2.6 we studied the theoretical prediction for the halo covariance. Now we will consider methods of estimating the covariance from real or simulated data.

\subsection{Constraining Parameters}

In order to constrain a set of parameters, it is necessary to determine $P(\Theta \mid \boldsymbol{O})$, the probability of having a certain parameter configuration $\Theta$ for a given observed data $\boldsymbol{O}$. We can work with the data to fit a set of parameters by computing a prediction for a set of parameters $\Theta$ and comparing this prediction with observations $\boldsymbol{O}$, instead of directly computing the parameters associated with a given observation. For instance, to estimate cosmological constraints from cluster abundance, we calculate the predicted number of clusters based on a set of cosmological parameters and compare it with the number counts measured. We cannot derive directly which set of parameters is associated with a certain observation. As a result, we end up computing 
the probability of having an observed data set $\boldsymbol{O}$, for a given parameter configuration $\Theta$. Using Bayes theorem, we can relate those two quantities as

$$
P(\boldsymbol{O}) P(\Theta \mid \boldsymbol{O})=P(\boldsymbol{O} \mid \Theta) P(\Theta)
$$

The first term on the left in Eq. 4.1 describes the probability of having the observed data. As $P(\boldsymbol{O})$ does not have any implications on the estimation of parameters, it can be left out in our analysis. The first term on the right is where the comparison between the prediction and the data is made, and is defined as the likelihood $\mathcal{L}$. The last term on the right concerns the a priori probability for the parameters to have certain values and is known as the prior $\Pi$. If there is no previous information about the values of the parameters, this term is one, meaning all values are equally possible. This prior knowledge can come from other cosmological probes (CMB, supernovae, two-point correlation functions of galaxies, quasar, lensing, etc.), from physical limits on the parameters (e.g. $\Omega_{b} \geq 1 \operatorname{or} \sigma_{8} \geq 0$ ), or from external calibrations derived in other data sets or in simulations (as the mass-proxy parameters). With these considerations, the probability of having a certain values for the parameters given a observation, known as the posterior $\mathcal{P}$, can be written as

$$
\mathcal{P}(\Theta \mid \boldsymbol{O})=\mathcal{L}(\boldsymbol{O} \mid \Theta) \Pi(\Theta)
$$

\subsubsection{Likelihood}

The likelihood describes how likely the observed data is, given a set of parameters, i.e. how close a theoretical prediction $\boldsymbol{T}(\Theta)$ with certain parameter configuration $\Theta$ is to an observation $\boldsymbol{O}$. If each of the observed data points is the result of a Gaussian realization of variance $\sigma_{i}^{2}$ around the "true" value, and the points are uncorrelated, the likelihood of each point $\mathcal{L}_{i}$ can be described by a Normal distribution and the total likelihood is the product of each $\mathcal{L}_{i}$ :

$$
\mathcal{L}=\prod_{i} \frac{1}{\sqrt{2 \pi \sigma_{i}^{2}}} \exp \left[-\frac{\left(O_{i}-T_{i}\right)^{2}}{2 \sigma_{i}^{2}}\right]=\frac{1}{\sqrt{\prod_{i} 2 \pi \sigma_{i}^{2}}} \exp \left[-\sum_{i} \frac{\left(O_{i}-T_{i}\right)^{2}}{2 \sigma_{i}^{2}}\right]
$$


This equation can be easily related to the $\chi^{2}$

$$
\chi^{2}=\sum_{i} \frac{\left(O_{i}-T_{i}\right)^{2}}{\sigma_{i}^{2}}=(\boldsymbol{O}-\boldsymbol{T}) \boldsymbol{C}^{-1}(\boldsymbol{O}-\boldsymbol{T})^{T}
$$

where $C$ in this case is a diagonal variance matrix with values $\sigma_{i}$. More generally the likelihood can be written as

$$
\mathcal{L}=\frac{1}{\sqrt{\operatorname{det}(2 \pi C)}} \exp \left[-\frac{\chi^{2}}{2}\right]
$$

In several cases of interest for modern cosmological surveys, the likelihood can be described by a Gaussian distribution, but with a covariance among the measurements, described by the $\boldsymbol{C}$ matrix. For these cases, the same form of the likelihood from Eq. 4.5 can be used, but using $\boldsymbol{C}$ as the full covariance matrix. Applying this formalism to the abundance of galaxy clusters, the observable is the number of clusters in each bin $N_{i}$ and the theoretical prediction is $\bar{m}_{i}$, given by Eq. 2.35. Each index $i$ represents the different measurements in several mass/redshift bins. The likelihood can be written as:

$$
\begin{aligned}
\mathcal{L} & =\frac{1}{\sqrt{2 \pi \operatorname{det}(\boldsymbol{C})}} \exp \left[\frac{(\boldsymbol{N}-\overline{\boldsymbol{m}}) \boldsymbol{C}^{-1}(\boldsymbol{N}-\overline{\boldsymbol{m}})^{T}}{2}\right] \\
& =\frac{1}{\sqrt{2 \pi \operatorname{det}(\boldsymbol{C})}} \exp \left[\sum_{i j} \frac{\left(N_{i}-\bar{m}_{i}\right) C_{i j}^{-1}\left(N_{j}-\bar{m}_{j}\right)}{2}\right],
\end{aligned}
$$

It is important to note that the formalism of Eq. 4.6 is valid only when the number of objects in each bin is large. When the expected number of objects in each bin is very small $(\lesssim 1)$, the distribution can no longer be considered Gaussian. In this limit, the appropriate likelihood is the Poisson distribution

$$
\mathcal{L}=\sum_{i} \frac{N_{i} ! \bar{m}_{i}^{N_{i}}}{\bar{m}_{i}}
$$

This can happen to galaxy clusters if the redshift range, angular size analyzed or the mass range is too small, or even if the minimum mass and redshift are large enough. However, the values that determine these limits always depend on all other specifications of the observed data considered, and each case must be carefully verified. 


\subsubsection{Marginalizing versus Fixing parameters}

An important question when dealing with multiple parameters $\Theta=\left\{\Theta_{1}, \Theta_{2}, \ldots, \Theta_{n}\right\}$ is how to estimate quantities related to only a single parameter $\Theta_{i}$. There are two possibilities for removing the dependency on all other parameters: fixing their values or marginalizing over them. Fixing the value of one specific parameter $\Theta_{i}$ means we have perfect knowledge about it, while marginalizing over $\Theta_{i}$ means that we consider the likelihood irrespective of the value of $\Theta_{i}$, e.g. we integrate the likelihood over all possible values of $\Theta_{i}$. Clearly, fixing $\Theta_{i}$ results in better constraints on all other parameters $\Theta_{j}(j \neq i)$, compared to marginalizing over $\Theta_{i}$. However, if we do not have informative prior information about a parameter, fixing its value can be dangerous, as it may bias the information about other parameters. To demonstrate this effect, let us consider the following likelihood of two parameters $\left(\Theta_{1}, \Theta_{2}\right)$ :

$$
\mathcal{L}\left(\Theta_{1}, \Theta_{2}\right) \propto \exp \left[-\frac{\Theta_{1}^{2}+\Theta_{2}^{2}+\Theta_{1} \Theta_{2}}{5}\right]
$$

It is clear that the best-fit, located at the maximum of the likelihood, occurs at $\Theta_{1}=\Theta_{2}=0$. In Fig. 4.1, we illustrate this likelihood with the best-fit values of $\left(\Theta_{1}, \Theta_{2}\right)$ represented by green lines. If we fixed $\Theta_{2}=-3$ (red solid line) the best-fit of $\Theta_{1}$ becomes 1.5 (red dashed line), a value considerably different from the true best fit.



Figure 4.1: Example of how fixing one parameter at a certain $\left(\Theta_{2}=-3\right.$ : red solid line $)$ can lead to a erroneous best fit for the other parameters $\left(\Theta_{1}=\max \left(\mathcal{L} \mid \Theta_{2}=-3\right)=2.4\right.$ : red dashed line). 
Furthermore, even if $\Theta_{i}$ is fixed at its best-fit value, the best-fit of the $\Theta_{o}$ may be different from that obtained by marginalizing over $\Theta_{i}$. For this example, consider the following likelihood:

$$
\mathcal{L}\left(\Theta_{1}, \Theta_{2}\right) \propto \exp \left[-\frac{\left(\Theta_{1}-3\right)^{2}+\left(\Theta_{2}-3\right)^{2}+\left(\Theta_{1} \Theta_{2}-9\right)^{2}}{5}\right]
$$

Here the overall best-fit occurs at $\Theta_{1}=\Theta_{2}=3$, shown in Fig. 4.2 as the red dashed lines. When we fix one parameter $\Theta_{i}$ at its overall best-fit value (=3), the likelihood resulting for the other parameter $\Theta_{j}$ (red dotted lines) conserves the same best-fit $(=3)$. However, if we marginalize over $\Theta_{i}$, the likelihood of $\Theta_{j}$ (blue solid line) has a different best-fit $(\approx 2.6)$. Moreover, comparing the red dotted lines and the blue solid line, we clearly see how fixing parameters results in tighter constraints than marginalizing over them. In this case, the mean value of the marginalized distributions $(\approx 3.1)$ is much closer to the overall best fit, hence reporting the mean value could bring information about the overall best fit. Clearly, fixing a parameter provides better constraints but also enforces a stronger assumption, while marginalizing over a parameter is a more conservative approach, resulting in weaker constraints.

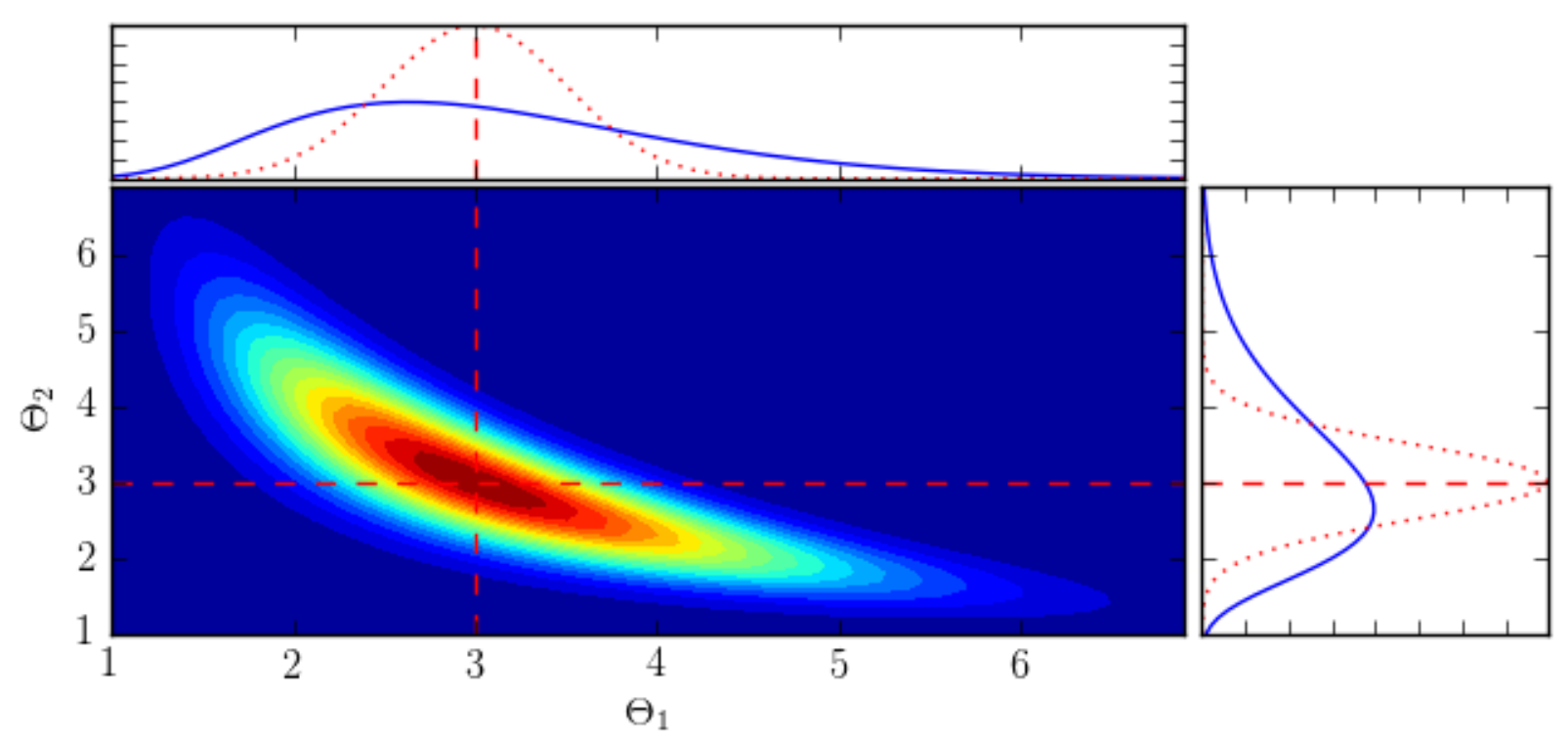

Figure 4.2: Example of how the best fit of a marginalized parameter (blue solid lines) can differ from the best fit overall (red dashed lines). 


\subsection{Fisher Matrix}

The Fisher Matrix (FM) (Pratt, 1976; Frieden, 2004), also known as the Fisher Information Matrix, estimates how much information can be extracted from a set of random variables with a given distribution. In the case of interest for cosmology, the random variable is an observable (two-point correlation function of galaxies, abundance of clusters, matter power spectrum, etc.), the distribution function is the likelihood and the information concerns the parameters of both cosmological and astrophysical nature.

An advantage of using Fisher Matrices is the ability to forecast constraints on cosmological (and non-cosmological) parameters, with a low computational cost, typically orders of magnitude faster MCMC and other parameter sampling methods. While the best sampling techniques require at least $\mathcal{O}\left(10^{4}\right)$ calculations of the likelihood, FM forecasts require only $\mathcal{O}\left(N_{\text {parameters }}\right)$. This method is also used e.g. to determine survey requirements on survey specifications according to an intended accuracy on the parameters.

The principle behind the Fisher Matrix method is that, at the peak of the likelihood, the distribution of all the parameters is Gaussian and the information is contained only in second order derivatives of the likelihood. Therefore, the validity of the FM results depends on how similar the actual parameter distribution is to a Gaussian. Additionally, as calculations are based around the peak of the likelihood, there is a dependency on the fiducial values chosen for the parameters. Ultimately, the results of the FM provide a simplified scenario of what can be expected from a survey, and offer intuition on the effects of multiple assumptions and parameters on cosmological constraints.

In this section and all subsections we will adopt the following notation: i) Vectors are represented by bold text font; ii) Greek indexes represent different parameters; iii) Latin indexes represent different mass/redshift/angular bins; Please observe that this notation differs from chapters 2 and 3 , and section 4.4 .

The Fisher Matrix is defined as

$$
F_{\alpha \beta}=-\left\langle\frac{\partial^{2} \ln \mathcal{L}(\Theta)}{\partial \Theta_{\alpha} \partial \Theta_{\beta}}\right\rangle
$$

where $\Theta_{\alpha}$ are parameters of the theoretical model, and it must be computed at a defined fiducial value, which will be considered the maximum of the likelihood. Once this matrix of derivatives is constructed, the procedure to obtain parameter constraints with different assumptions is quite 
simple. The final error on a parameter $\Theta_{\alpha}$, after marginalizing over all the others is given by $\sigma\left(\Theta_{\alpha}\right)=\left[F^{-1}\right]_{\alpha \alpha}^{1 / 2}$. If there is a prior knowledge on a parameter $\Theta_{\alpha}$ at the level of $\sigma_{\text {prior }}\left(\Theta_{\alpha}\right)$, it can be added to the Fisher Matrix by $F_{\alpha \beta} \rightarrow F_{\alpha \beta}+\sigma_{\text {prior }}\left(\Theta_{\alpha}\right)^{-2} \delta_{\alpha \beta}$. To fix a certain parameter $\Theta_{\alpha}$, one can remove the line and column corresponding to $\Theta_{\alpha}$, or equivalently add an infinite prior to $F_{\alpha \alpha}$ (i.e. a zero-uncertainty prior).

Once the Fisher Matrix is calculated and inverted, the probability contours for each pair of parameters $\alpha$ and $\beta$ can be described as ellipses with parameters $a, b, \theta$ given by

$$
\begin{aligned}
a^{2}=\frac{F_{\alpha \alpha}^{-1}+F_{\beta \beta}^{-1}}{2}+\sqrt{\left(\frac{F_{\alpha \alpha}^{-1}-F_{\beta \beta}^{-1}}{2}\right)^{2}+\left(F_{\alpha \beta}^{-1}\right)^{2}} \\
b^{2}=\frac{F_{\alpha \alpha}^{-1}+F_{\beta \beta}^{-1}}{2}-\sqrt{\left(\frac{F_{\alpha \alpha}^{-1}-F_{\beta \beta}^{-1}}{2}\right)^{2}+\left(F_{\alpha \beta}^{-1}\right)^{2}} \\
\tan 2 \theta=\frac{2 F_{\alpha \beta}^{-1}}{F_{\alpha \alpha}^{-1}-F_{\beta \beta}^{-1}}
\end{aligned}
$$

where $a$ and $b$ are the major and minor axes of the ellipse and $\theta$ the inclination of the major axis relative to the $\alpha$ axis. The $68 \%(1 \sigma)$ confidence level occurs multiplying $a$ and $b$ by the value of two-parameter $\chi^{2}$ variation $\sqrt{\Delta \chi_{(68 \%)}^{2}} \approx 1.52$ and the $95 \%(2 \sigma)$ confidence level by $\sqrt{\Delta \chi_{(95 \%)}^{2}} \approx 2.48$.

In the context of dark matter halo abundance, the Fisher Matrix formalism can be reduced to even simpler equations that only require first derivatives of the halo number counts and covariance. On the next sections, we present the computations for FM constraints with halo abundance and covariance, and also show how to estimate the bias on cosmological parameters caused by unpredicted deviations on observations.

\subsubsection{Gaussian Likelihood}

For the Gaussian case (Eq. 4.6) with sample covariance matrix $S$, the theoretical prediction for halo abundance $\overline{\boldsymbol{m}}$ and number of halos measured or observed $\boldsymbol{N}$, the logarithm of likelihood 
becomes

$$
\ln \mathcal{L}=-\frac{1}{2}\left[\ln 2 \pi+\ln \operatorname{det} \boldsymbol{S}+(\overline{\boldsymbol{m}}-\boldsymbol{N}) \boldsymbol{S}^{-1}(\overline{\boldsymbol{m}}-\boldsymbol{N})^{T}\right]
$$

Using the matrix properties:

$$
\left\{\begin{array}{l}
\ln \operatorname{det}(\boldsymbol{S})=\operatorname{Tr}[\ln (\boldsymbol{S})] \\
\ln \boldsymbol{S}_{, \alpha}=\boldsymbol{S}^{-1} \boldsymbol{S}_{, \alpha}
\end{array}\right.
$$

and the results of the averages:

$$
\left\{\begin{array}{l}
\left\langle\bar{m}_{i}-N_{i}\right\rangle=\bar{m}_{i}-\left\langle N_{i}\right\rangle=0, \\
\left\langle\left(\bar{m}_{i}-N_{i}\right)\left(\bar{m}_{j}-N_{j}\right)\right\rangle=S_{i j}
\end{array}\right.
$$

one obtains the following Fisher Matrix (Tegmark et al., 1997; Tegmark, 1997):

$$
F_{\alpha \beta}=\overline{\boldsymbol{m}}_{, \alpha} \boldsymbol{S}^{-1} \overline{\boldsymbol{m}}_{, \beta}{ }^{T}+\frac{1}{2} \operatorname{Tr}\left[\boldsymbol{S}^{-1} \boldsymbol{S}_{, \alpha} \boldsymbol{S}^{-1} \boldsymbol{S}_{, \beta}\right]
$$

where the partial derivatives are represented by the comma separation $\left(\partial X / \partial \Theta_{\alpha} \rightarrow X, \alpha\right)$.

\subsubsection{Poisson Likelihood}

The Poisson distribution describes shot-noise statistics for cases when the expected signal of a measurement is very low. For a Poisson likelihood, the resulting Fisher Matrix is

$$
F_{\alpha \beta}=\sum_{i=1}^{b} \frac{\bar{m}_{i, \alpha} \bar{m}_{i, \beta}}{\bar{m}_{i}}=\overline{\boldsymbol{m}}_{, \alpha} \boldsymbol{M}^{-1} \overline{\boldsymbol{m}}_{, \beta}{ }^{T}
$$

where the diagonal covariance matrix for the Poisson case was defined as $M_{i j}=\bar{m}_{i} \delta_{i j}$, reminding once again that Greek indices represent different parameters while Latin indices represent different mass/redshift/angular bins. The fact that this matrix is diagonal establishes that there is no covariance between the different bins. 


\subsubsection{Interpolated Likelihood}

The expected number of galaxy clusters can be too large to be described by the Poisson limit $(\bar{m} \rightarrow 0)$ and not large enough to obey the Gaussian distribution perfectly $(\bar{m} \rightarrow \infty)$. In this case, the distribution should be somewhere in between.

From the functional forms of Eq. 4.17 and Eq. 4.18, one can work with a convoluted likelihood which approximates the resulting Fisher Matrix (Lima \& Hu, 2004). In order to be statistically consistent, this new matrix should approach the Poisson and Gaussian cases in the appropriate limits. Defining the total covariance as the sum of the Poisson and Gaussian covariances $\boldsymbol{C}=\boldsymbol{M}+\boldsymbol{S}$, one may interpolate the Fisher Matrix as

$$
F_{\alpha \beta}=\overline{\boldsymbol{m}}_{, \alpha} \boldsymbol{C}^{-1} \overline{\boldsymbol{m}}_{, \beta}^{T}+\frac{1}{2} \operatorname{Tr}\left[\boldsymbol{C}^{-1} \boldsymbol{S}_{, \alpha} \boldsymbol{C}^{-1} \boldsymbol{S}_{, \beta}\right]
$$

In the limit $\bar{m} \rightarrow \infty$, the Poisson distribution becomes Gaussian and the Fisher Matrix results in the convolution of two Normal distributions with total covariance $\boldsymbol{C}=\boldsymbol{M}+\boldsymbol{S}$. For small number counts $(\bar{m} \lesssim 1)$, the sample variance $S \sim \bar{m}^{2}$ is negligible compared to the Poisson variance. The second term of Eq. 4.19 is of order $\bar{m}^{2}$ while the first term is of order $\bar{m}$, recovering the Poisson case.

\subsubsection{Bias}

Finally, we must consider consider that variations on the number counts and on the sample covariance, relative to their values in the fiducial model, induces a systematic error or bias on the parameters constrained. For a given $\Delta \overline{\boldsymbol{m}}$ on the number counts and $\Delta \boldsymbol{S}$ on the sample covariance, the bias $b\left(\Theta_{\alpha}\right)=\delta \Theta_{\alpha}$ on the derived parameter $\Theta_{\alpha}$ is given by (Amara \& Réfrégier, 2008; Erickson et al., 2011)

$$
b\left(\Theta_{\alpha}\right)=F_{\alpha \beta}^{-1}\left\{\overline{\boldsymbol{m}}_{, \beta} \boldsymbol{C}^{-1} \Delta \overline{\boldsymbol{m}}+\frac{1}{2} \operatorname{Tr}\left[\boldsymbol{C}^{-1} \boldsymbol{S}_{, \beta} \boldsymbol{C}^{-1} \Delta \boldsymbol{S}\right]\right\} .
$$

This can be used for assessing the bias on inferred cosmological parameters when neglecting e.g. the inclusion of selection function parameters, given that the true counts in the fiducial model require these additional parameters. 


\subsection{MCMC methods}

The Monte Carlo Markov Chain (MCMC) (Metropolis et al., 1953) is a method for sampling from probability distributions. It is extremely helpful for the estimation of cosmological parameters, since it can map parameter spaces with a large number of dimensions faster and more accurately than grid methods. Unlike the Fisher Matrix approach, the MCMC technique does not assume a Gaussian correlation for the parameter posterior, and instead includes all distribution moments and cross-correlations. In most cases the likelihood and priors are frequently assumed to be Gaussian, though for clusters as discussed above the likelihood is a convolution of Gaussian and Poisson distributions.

The Metropolis-Hastings algorithm for MCMC sampling has been used extensively in several fields, including physics and cosmology (Christensen et al., 2001; Lewis \& Bridle, 2002; Dunkley et al., 2005). The method consists of a random walk in parameter space, with steps taken according to the posterior and a criterion for accepting a proposed move.

The basic approach of this MCMC consists of:

1. From a point $\Theta$ in parameter space, propose a random step $\Theta_{S}$

(a) If $\mathcal{P}\left(\Theta_{s}\right)>\mathcal{P}(\Theta)$ : take the step $\left(\Theta \rightarrow \Theta_{s}\right)$

(b) Else: Generate a random number $R \in[0: 1]$

i. If the ratio $\mathcal{P}\left(\Theta_{S}\right) / \mathcal{P}(\Theta)>R$ : take the step $\left(\Theta \rightarrow \Theta_{S}\right)$

ii. Else: Remain at the original point $\Theta$

2. Repeat step 1 until the distribution of the parameters converge

The basic idea is that the proposed step is always accepted when the new point has a higher posterior. However, it also includes the possibility of moving to a point of lower posterior with a probability proportional to ratio of the new posterior relative to the old one. This leads the chain to search for the most likely values of the parameters and also to appropriately sample the surrounding regions around the best-fit. After an initial phase in which the chain steps approach the best-fit region (burn-in), it will sample the parameter space around the best-fit, and the final point distribution will converge to the actual posterior distribution.

However, the whole chain will not necessarily have the same distribution as the posterior. For instance, in cases of uninformative priors, it is unlikely that the initial parameter values $\Theta_{i}$ will be close to the peak of the posterior. The chain will then initially make a path leading 
to the most likely regions. This path will not be sampling the likelihood distribution, for, in general, the ratio of acceptance of steps $\alpha$ is practically 1 in the direction of the most likely point and $\alpha \rightarrow 0$ for other directions, leading to a nearly monotonic increase of the likelihood. In order to correct the chain for a representative sampling of the likelihood, it is usually applied a burn-in. This process consists of removing the initial non-representative steps from the chain, characterized by a semi-monotonic growth of the likelihood. This assures that the remaining chain has converged and can be properly used for best-fits and errors inference. There are other methods to check for the convergence by analyzing the power spectrum of the MCMC chain (Dunkley et al., 2005).

One drawback of MCMC methods is the impossibility of parallelizing steps due to the nature of a Markov Chain, as each step depends on the immediately previous step. Therefore having multiple computer processors does not speed up the computation of one chain. In principle, it is possible to combine two distinct chains that have reached convergence. This would allow the sampling to be parallelized by having multiple chains. However, one must be certain that each chain has converged, which is not simple to determine. Thus, the combination of multiple MCMC chains has to be done carefully.

The algorithm used in this work is the affine invariant sampler (Foreman-Mackey et al., 2013), a variation of MCMC that can be parallelized at its core. This method consists of having multiple walkers in parameter space, which move all together, taking into account each other's positions. The calculation of the likelihood in each walker can be done independently in parallel. The actual code is emcee, a complete python package designed for a wide range of applications.

The mapping process of this method works by having an even number of walkers. The initial position of the walkers are sorted randomly with a Gaussian distribution around a point in the parameter space, according to initial the value and scatter of the parameters provide. Then the walkers are divided randomly into two same sized groups $S_{0}$ and $S_{1}$. Each walker from $S_{0}$ selects randomly a walker from $S_{1}$, and sorts a new point in the line uniting the pair.The steps are accepted according to the same conditions from the usual MCMC methods, and the computation for each walker can be computed in parallel. Finally, the walkers from $S_{1}$ can be updated based on the new positions of $S_{0}$. This updating of both groups is considered a step of the walkers.

Using this approach it is possible to have a convergence of the walkers with a much smaller 
number of steps than with the usual MCMC methods. The computation in each step is $N_{\text {walkers }}$ times slower than MCMC. However, as parallelization can be used, it does not affect the total time to sample the parameter space.

\subsection{Estimation of Sample Covariance from Observations}

Accurately determining uncertainties on the abundance of halos is extremely important for constraining cosmological parameters. The variance of the counts measurements is directly linked to the size of the final constraints. Additionally, the covariance between different masses and/or redshifts contain cosmological information, and can improve the constraining process. As described in section 2.6, the covariance can be theoretically estimated given a cosmology. In fact, using a theoretical covariance with cosmological dependency can greatly improve constraint results as will be shown in 5.2. Unfortunately, theoretical calculations for the covariance involve a two-dimensional integral over Bessel functions, which can have a much higher computational cost than estimating only the halo number counts. Furthermore, fixing the covariance matrix can lead to a systematic bias of the cosmological constraint if the cosmology used on the theoretical covariance matrix is not the same derived from the data. Therefore, accurately estimating the covariance from the data is very important.

In this section we will present three methods to estimate the covariance from the data. All of these techniques work by making angular sub-samples of the initial data. To avoid confusion between the sub-samples generated by the methods and the mass/redshift bin, we will use Greek indexes to describe a specific mass and redshift bin $\alpha=\{M, z\}$ and Latin indices for the subsamples, differently from the notation used in chapters 2 and 3 , and section 4.2 .

When working with simulations, a usual method is to make several realizations based on a underlying cosmology. Hence, there will be multiple samples of the Universe that can be used to measure the covariance of the total observed region.

\subsubsection{Sub-sampling}

This is the simplest method, where the data is split into $N$ smaller sub-samples with the same angular size and shape. We will refer to each of these sub-samples as a pixel so as to avoid confusion with the mass/redshift bins. The number counts is measured in each pixel and the total covariance will be the covariance between pixels. Given two mass and redshift bins $\alpha$ and 
$\beta$, the covariance can be computed based on the halo counts $m$ as

$$
C_{\alpha \beta}=\left\langle m_{\alpha} m_{\beta}\right\rangle-\left\langle m_{\alpha}\right\rangle\left\langle m_{\beta}\right\rangle=\frac{1}{N-1}\left[\sum_{i}^{N} m_{\alpha i} m_{\beta i}-\frac{1}{N} \sum_{j}^{N} m_{\alpha j} \sum_{k}^{N} m_{\beta k}\right] .
$$

Using this sub-sampling, the resulting covariance computed is valid for the smaller sub-samples. One could try to estimate the covariance of the total sample by re-scaling 4.21 with the total number of samples $N$. This assumption does not consider the cosmic variance in its entirety, which has a scale dependency. Nonetheless, it can provide a rough estimation, as will be studied in section 6.1.

\subsubsection{Jackknife Sub-sampling}

The Jackknife Sub-sampling (JK) method was developed by Quenouille (1949) and consists of splitting the original data into $N$ parts. However, each new sample is constructed by removing one of the smaller parts, resulting in $N$ samples composed of $N-1$ parts. Therefore the mean value of halo abundance $\left\langle m_{\alpha}\right\rangle_{i}$ in each sample can be computed by removing the $i$ th piece:

$$
\left\langle m_{\alpha}\right\rangle_{i}=\frac{1}{N-1} \sum_{j \neq i}^{N} m_{\alpha j}
$$

With the intent to recover the total covariance of the sample, the JK estimation is given by

$$
C_{\alpha \beta}=\frac{N-1}{N} \sum_{i}^{N}\left(\left\langle m_{\alpha}\right\rangle_{i}-\left\langle m_{\alpha}\right\rangle\right)\left(\left\langle m_{\beta}\right\rangle_{i}-\left\langle m_{\beta}\right\rangle\right)
$$

where $\left\langle m_{\alpha}\right\rangle$ is the mean value using all $N$ samples:

$$
\left\langle m_{\alpha}\right\rangle=\frac{1}{N} \sum_{i}^{N} m_{\alpha i}
$$

This technique is also purely statistical and does not consider cosmic covariance in its entirety. Its validity for cluster covariances will be checked in section 6.1.

\subsubsection{Bootstrap Sub-sampling}

The technique of Bootstrap Sub-sampling (BS) was developed by Efron (1992), inspired by JK methods. On BS, the data is divided into $N$ parts and samples are created by randomly selecting 
$N$ pieces, allowing for repetition. Each sample $i$ now has the total number of clusters $m_{\alpha, i}^{B S}$ given by

$$
m_{\alpha i}^{B S}=\sum_{j \sim[1, N]}^{N} m_{\alpha j}
$$

where the sum is over the $N$ random pieces composing the sample. This way an arbitrary number $N_{B S}$ of samples can be created and the measurements of the covariance is made with samples of the same size of the original data. For a given number of BS samples $N_{B S}$, the covariance can be computed in a similar manner to Eq. 4.21

$$
C_{\alpha \beta}=\frac{1}{N_{B S}-1}\left[\sum_{i}^{N_{B S}} m_{\alpha i}^{B S} m_{\beta i}^{B S}-\frac{1}{N_{B S}} \sum_{j}^{N_{B S}} m_{\alpha j}^{B S} \sum_{k}^{N_{B S}} m_{\beta k}^{B S}\right]
$$

As $N_{B S} \rightarrow \infty$, the covariance should converge to the correct value. Again this estimation does not consider cosmic variance fully and its limitations will be tested in section 6.1.

\subsubsection{Multiple Simulation Sampling}

This approach is applicable to simulations only, where the cosmological and physical conditions are know. It consists of generating several realizations of the simulation and computing the covariance among them. For this estimation, the covariance can be computed with the same equation from Sub-sampling (Eq. 4.21), but using the Latin indices to represent the $N$ realizations. In this case, the estimated covariance must be valid on all scales of the simulation, whereas in Sub-sampling it was valid only on the smaller scales defined by sub-samples. 


\section{Chapter 5}

\section{Theoretical Results}

In this chapter we will discuss results from a theoretical standpoint only. The main goal is to study part of the formalism for the prediction of the abundance of galaxy clusters and its power to constrain cosmology. In particular, we will analyze the mass-proxy distribution and the inverse proxy-mass relation and how to derive one from the other. Cosmological constraints will be obtained using the Fisher Matrix formalism, exploring first in detail constraining cosmology with the dark matter halos. Afterwards, the optical effects will be introduced, with emphasis on completeness and purity. This latest work led to a scientific paper submitted for publication (Aguena \& Lima, 2016).

\subsection{Mass-Observable relation}

As discussed in previous chapters, one of the most important steps for constraining cosmology with galaxy clusters is to either have an accurate measurement of the cluster mass (via lensing, $\mathrm{X}$-Ray or SZ) or to use a mass-proxy relation and account for the scatter of this relation on the theoretical prediction for cluster abundance. Here we will discuss the properties of the mass-observable relation used to go from predictions of the abundance of ideal dark matter halos to actually detected galaxy cluster. Specifically, the gaussianity of the mass-observable distribution and the consequent observable-mass distribution. For simplicity of the units used, the mass proxy $Y$ will be converted to an "observable" mass $M^{\text {obs }}(Y)$. If we make the conversion by simply re-scaling the proxy $Y$ to have mass units, the resulting volume in probability density 
space $d V_{p}\left(M, M^{\text {obs }}\right)$ (Eq. 3.7) can be rewritten as

$$
\begin{aligned}
d V_{p}\left(M, M^{\mathrm{obs}}\right) & \equiv \\
P\left(M \mid M^{\mathrm{obs}}\right) d \ln M \frac{d \bar{n}}{d \ln M^{\mathrm{obs}}} d \ln M^{\mathrm{obs}} & =P\left(M^{\mathrm{obs}} \mid M\right) d \ln M^{\mathrm{obs}} \frac{d \bar{n}}{d \ln M} d \ln M
\end{aligned}
$$

Here it is important to note that, as has been defined in chapter 3.3.2, $P\left(M \mid M^{\mathrm{obs}}\right)$ and $P\left(M^{\mathrm{obs}} \mid M\right)$ actually represent the probabilities in log space of mass and proxy.

In recent works (Erickson et al., 2011; Saro et al., 2015; Farahi et al., 2016; Baxter et al., 2016, Simet et al., 2017), the prediction for the abundance of galaxy clusters was constructed using both $P\left(M \mid M^{\text {obs }}\right)$ and $P\left(M^{\text {obs }} \mid M\right)$ and assuming these distributions to be Gaussian. However, this issue is not discussed in much detail, sometimes leading to confusing statements. Our goal here is to have a clear understanding of the use of these distributions.

When considering the conversion from predictions with the halo mass $M$ to predictions based on the cluster observable mass $M^{\text {obs }}$, the first step is to comprehend what each $P$ distribution describe. $P\left(M \mid M^{\text {obs }}\right)$ is the mass $M$ distribution of dark matter halos connected to galaxy clusters with observable mass $M^{\text {obs }}$. That means, to map out all the halos associated to clusters of observable mass $M^{\text {obs }}$. Meanwhile, $P\left(M^{\text {obs }} \mid M\right)$ is the observed mass $M^{\text {obs }}$ distribution of the galaxy clusters clusters associated to dark matter halo of mass $M$. In other words, $P\left(M \mid M^{\text {obs }}\right)$ is the distribution along horizontal lines of $M \times M^{\text {obs }}$ diagrams such as in Fig. 5.1, whereas $P\left(M^{\text {obs }} \mid M\right)$ is the distribution along vertical lines.

\section{Gaussian distributions}

It is important to understand that $P\left(M \mid M^{\mathrm{obs}}\right)$ is a function of $M$ for a given predetermined $M^{\text {obs }}$ and $P\left(M^{\mathrm{obs}} \mid M\right)$ is the function of $M^{\text {obs }}$ for a given $M$. In principle, one might suppose it is possible for both distributions to be Gaussian with respect to the log of their variables:

$$
\begin{gathered}
P\left(M^{\mathrm{obs}} \mid M\right)=\frac{1}{\sqrt{2 \pi \sigma_{\ln M^{\mathrm{obs}}}^{2}(M)}} \exp \left[\frac{\left(\ln M^{\mathrm{obs}}-\ln M-\ln M_{\text {bias }}^{\mathrm{obs}}(M)\right)^{2}}{2 \sigma_{\ln M^{\mathrm{obs}}}^{2}(M)}\right], \\
P\left(M \mid M^{\mathrm{obs}}\right)=\frac{1}{\sqrt{2 \pi \sigma_{\ln M^{2}}^{2}\left(M^{\mathrm{obs}}\right)}} \exp \left[\frac{\left(\ln M-\ln M^{\mathrm{obs}}-\ln M_{\text {bias }}\left(M^{\mathrm{obs}}\right)\right)^{2}}{2 \sigma_{\ln M^{2}}^{2}\left(M^{\mathrm{obs}}\right)}\right] .
\end{gathered}
$$

Eq. 5.2 describes a Gaussian distribution on $M^{\text {obs }}$ with the bias $\ln M_{\text {bias }}^{\text {obs }}$ and scatter $\sigma_{\ln M^{\text {obs }}}^{2}$ parameters dependent on $M$. Likewise, the exact opposite happens in Eq. 5.3. These dependencies 
on scatter an biases are connected, as both distributions are linked by Eq. 5.1. In fact, we can compute $P\left(M \mid M^{\text {obs }}\right)$ for a known $P\left(M^{\text {obs }} \mid M\right)$ and mass function $d \bar{n} / d \ln M$ as

$$
P\left(M \mid M^{\mathrm{obs}}\right)=\frac{P\left(M^{\mathrm{obs}} \mid M\right) \frac{d \bar{n}}{d \ln M}}{\frac{d \bar{n}}{d \ln M^{\mathrm{obs}}}}=\frac{P\left(M^{\mathrm{obs}} \mid M\right) \frac{d \bar{n}}{d \ln M}}{\int_{-\infty}^{\infty} d \ln M P\left(M^{\mathrm{obs}} \mid M\right) \frac{d \bar{n}}{d \ln M}}
$$

By doing so, we can check the validity of assuming both distributions to be Gaussian simultaneously, i.e. the functional forms of Eq. 5.2 and Eq. 5.3. Note that if e.g. $P\left(M^{\text {obs }} \mid M\right)$ is a Gaussian, then clearly $P\left(M \mid M^{\text {obs }}\right)$ is not a perfect Gaussian, given that the mass function is roughly a power law at low masses with an exponential cut-off at high masses (see Fig. 2.1. Thus, it is be useful to check how well $P\left(M \mid M^{\text {obs }}\right)$ can still be approximated by a Gaussian in this case.

As an example, let us analyze a case where $P\left(M^{\text {obs }} \mid M\right)$ is Gaussian, described by Eq. 5.2 with $\ln M_{\text {bias }}^{\text {obs }}=0, \sigma_{\ln M^{\text {obs }}}=0.3 \ln 10 \approx 0.7$ and the mass function $d \bar{n} / d M$ is computed using Tinker et al. (2008) in a flat $\Lambda \mathrm{CDM}$ cosmology with Planck fiducial values (Table 1.1). The resulting joint probability distribution $d V_{p}\left(M, M^{\text {obs }}\right)$ is shown in Fig. 5.1. The black solid line is the diagonal $l_{d}: M^{\text {obs }}=M(\mathrm{y}=\mathrm{x})$, the gray dashed line is given by $l_{M_{\max }}: M=M_{\max }\left(M^{\mathrm{obs}}\right)$, the mass $M$ at which $d V_{p}$ is maximum at given $M^{\text {obs }}$ :

$$
\max _{M}\left[P\left(M^{\mathrm{obs}} \mid M\right) \frac{d \bar{n}}{d \ln M}(M)\right]=P\left(M^{\mathrm{obs}} \mid M_{\max }\right) \frac{d \bar{n}}{d \ln M}\left(M_{\max }\right)
$$

and the gray dotted line is given by $l_{M_{\max }^{\text {obs }}}$, analogous to $l_{M_{\max }}$ but switching $M$ and $M^{\text {obs }}$. The curve $l_{M_{\max }}$ is defined by finding the maximum values on horizontal lines of Fig. 5.1, while $l_{M_{\max }^{\text {obs }}}$ is defined by finding the maximum values on vertical lines. If both $P\left(M^{\text {obs }} \mid M\right)$ and $P\left(M \mid M^{\text {obs }}\right)$ were Gaussian with no bias, we should expect all three lines $l_{M_{\max }}, l_{M_{\max }^{\text {obs }}}$ and $l_{d}$ to coincide. In fact $l_{M_{m a x}^{\text {obs }}}$ and $l_{d}$ do coincide, as by construction the maximum of $P\left(M^{\text {obs }} \mid M\right)$ occurs at the diagonal for an unbiased Gaussian. However, $l_{M_{\max }}$ has lower values of $M$ compared to $l_{d}$ at a given $M^{\text {obs }}$. This happens because $d \bar{n} / d \ln M$ is higher at lower masses, weighting down $P\left(M^{\text {obs }} \mid M\right)$ at all masses. This results in a negative bias for $P\left(M \mid M^{\text {obs }}\right)$ given an unbiased $P\left(M^{\text {obs }} \mid M\right)$.

If we wish to enforce a $\log$-Gaussian fit for the resulting $P\left(M \mid M^{\text {obs }}\right)$ we only need to com- 




Figure 5.1: Probability density space $d V_{p}\left(M, M^{\text {obs }}\right)$ (Eq. 5.1 for a Gaussian $P\left(M^{\text {obs }} \mid M\right)$ with fixed scatter and no bias. The black line is the diagonal, the gray dashed line is defined by $l_{M_{\max }}$ (Eq. 5.5) and the gray dotted line is given by $l_{M_{\max }^{\text {obs }}}$ (it coincides completely with the diagonal). 
pute $\sigma_{\ln M}\left(M^{\mathrm{obs}}\right)$ and $\ln M_{\text {bias }}\left(M^{\mathrm{obs}}\right)$ from the measured first two moments of the distribution:

$$
\begin{aligned}
\ln M_{\text {bias }}\left(M^{\mathrm{obs}}\right) & =p_{1}\left(M^{\mathrm{obs}}\right)-\ln M^{\mathrm{obs}} \\
\sigma_{\ln M}^{2}\left(M^{\mathrm{obs}}\right) & =p_{2}\left(M^{\mathrm{obs}}\right)-p_{1}^{2}\left(M^{\mathrm{obs}}\right)
\end{aligned}
$$

where the $k^{\text {th }}$ moment of $P\left(M \mid M^{\text {obs }}\right)$ can be calculated by

$$
p_{k}\left(M^{\mathrm{obs}}\right)=\int_{-\infty}^{\infty} d \ln M P\left(M \mid M^{\mathrm{obs}}\right)(\ln M)^{k}=\frac{\int_{-\infty}^{\infty} d \ln M P\left(M^{\mathrm{obs}} \mid M\right) \frac{d \bar{n}}{d \ln M}(\ln M)^{k}}{\int_{-\infty}^{\infty} d \ln M P\left(M^{\mathrm{obs}} \mid M\right) \frac{d \bar{n}}{d \ln M}}
$$

The resulting parameters of the $P\left(M \mid M^{\text {obs }}\right)$ can be seen in Fig. 5.2. The amplitude of the bias $\ln M_{\text {bias }}$ increases at higher $M^{\text {obs }}$, following the distance between $l_{M_{\max }^{\mathrm{obs}}}$ and $l_{d}$. The scatter $\sigma_{\ln M}$ goes from a plateau with value of $\sigma_{\ln M^{\text {obs }}} \approx 0.7$ at low masses to a rapid decrease at high masses.
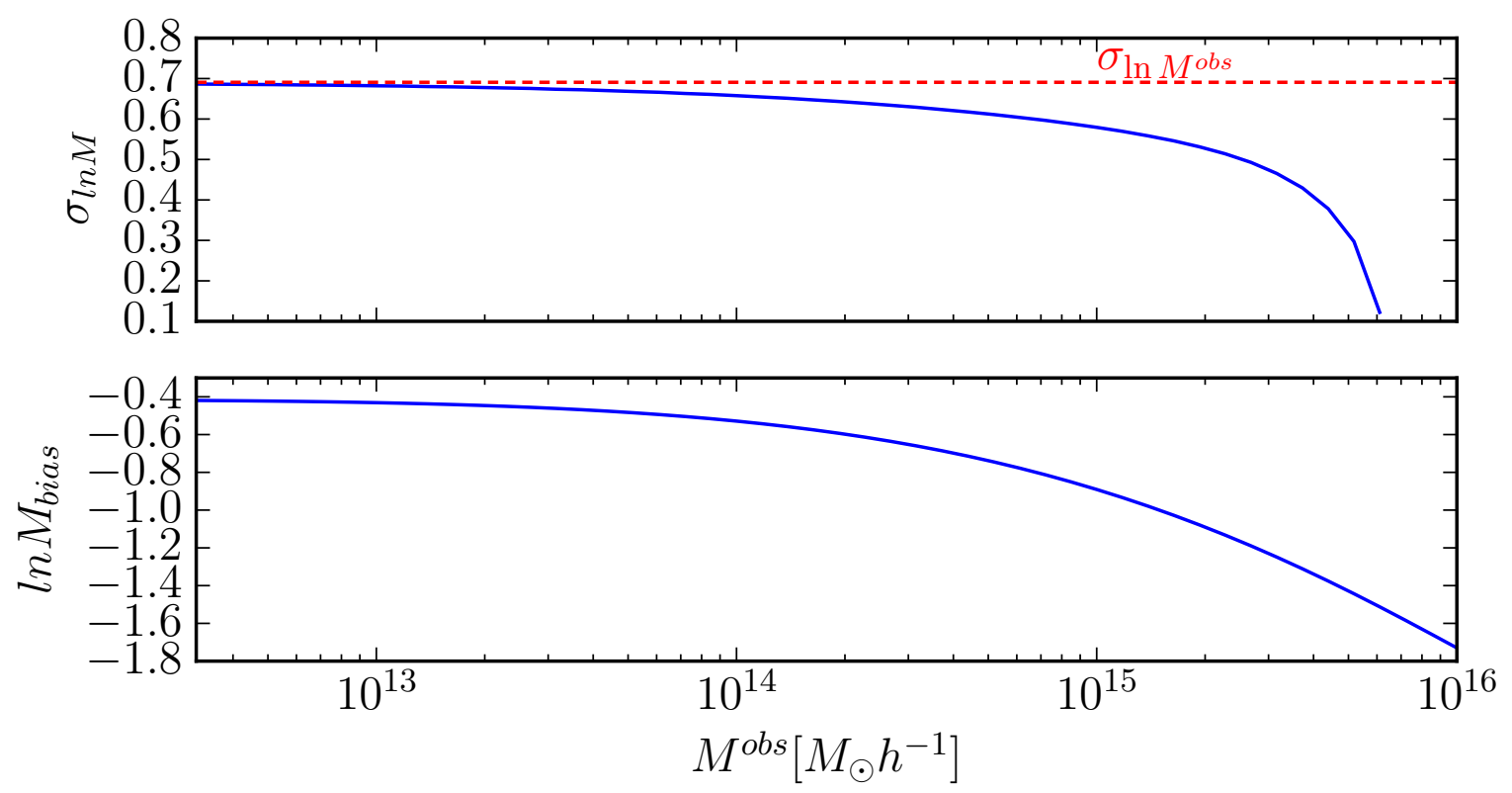

Figure 5.2: Mass scatter $\sigma_{\ln M}$ and mass bias $\ln M^{\text {bias }}$ of $P\left(M \mid M^{\text {obs }}\right)$ resulting from a Gaussian $P\left(M^{\text {obs }} \mid M\right)$ with fixed scatter and no bias.

To check how close $P\left(M \mid M^{\text {obs }}\right)$ is to a Gaussian distribution, we display in Fig. 5.3 the distribution $P\left(M^{\text {obs }} \mid M\right)$ (black dashed line), the resulting $P\left(M \mid M^{\text {obs }}\right)$ (blue solid line), and a Gaussian distribution (red dotted line) with scatter $\sigma_{\ln M}$ and mass bias $\ln M^{\text {bias }}$ fitted in Fig. 5.2, concerning several fixed values of $M^{\text {obs }}$. Not only $P\left(M \mid M^{\text {obs }}\right)$ resembles a Gaussian distribution, but it can also be well described by a Gaussian (red dotted line). A quantitative analysis of the Gaussianity of $P\left(M \mid M^{\mathrm{obs}}\right)$ will be presented in the next section. 




Figure 5.3: Gaussian distribution $P\left(M^{\text {obs }} \mid M\right)$ (black dashed line) with fixed scatter and no bias, the resulting $P\left(M \mid M^{\text {obs }}\right)$ (blue solid line), and Gaussian distribution (red dotted line) with scatter $\sigma_{\ln M}$ and mass bias $\ln M^{\text {bias }}$ fitted in Fig. 5.2 for several fixed values of $M^{\text {obs }}$.

\section{Testing Gaussianity}

We can check the validity of $P\left(M \mid M^{\text {obs }}\right)$ being Gaussian by comparing higher order moments $(>2)$ with the known Gaussian higher order moments. The Gaussian distribution moments of order grater than 2 can be calculated based on $p_{1}$ and $p_{2}$ (or $\sigma^{2}$ ):

$$
\left\{\begin{array}{l}
G p_{3}=p_{1}^{3}+3 p_{1} \sigma^{2}, \\
G p_{4}=p_{1}^{4}+6 p_{1}^{2} \sigma^{2}+3 \sigma^{4}, \\
G p_{5}=p_{1}^{5}+10 p_{1}^{3} \sigma^{2}+15 p_{1} \sigma^{4}, \\
G p_{6}=p_{1}^{6}+15 p_{1}^{4} \sigma^{2}+45 p_{1}^{2} \sigma^{4}+15 \sigma^{6}, \\
G p_{7}=p_{1}^{7}+21 p_{1}^{5} \sigma^{2}+105 p_{1}^{3} \sigma^{4}+105 p_{1} \sigma^{6}, \\
G p_{8}=p_{1}^{8}+28 p_{1}^{6} \sigma^{2}+210 p_{1}^{4} \sigma^{4}+420 p_{1}^{2} \sigma^{6}+105 \sigma^{8} .
\end{array}\right.
$$


The results of this comparison are shown in Fig. 5.4. where we computed up to the 8th moment. The relative difference does increase with $M^{\text {obs }}$. However, the highest value of the relative difference of the moments computed (the 8th moment at $M^{\text {obs }}=10^{16} M_{\odot} h^{-1}$ ) is of order $1 \%$. Hence, a Gaussian $P\left(M^{\text {obs }} \mid M\right)$ with fixed scatter and no bias seems to result - in very good approximation - in a Gaussian $P\left(M \mid M^{\text {obs }}\right)$, but with scatter and bias dependent of $M^{\text {obs }}$.

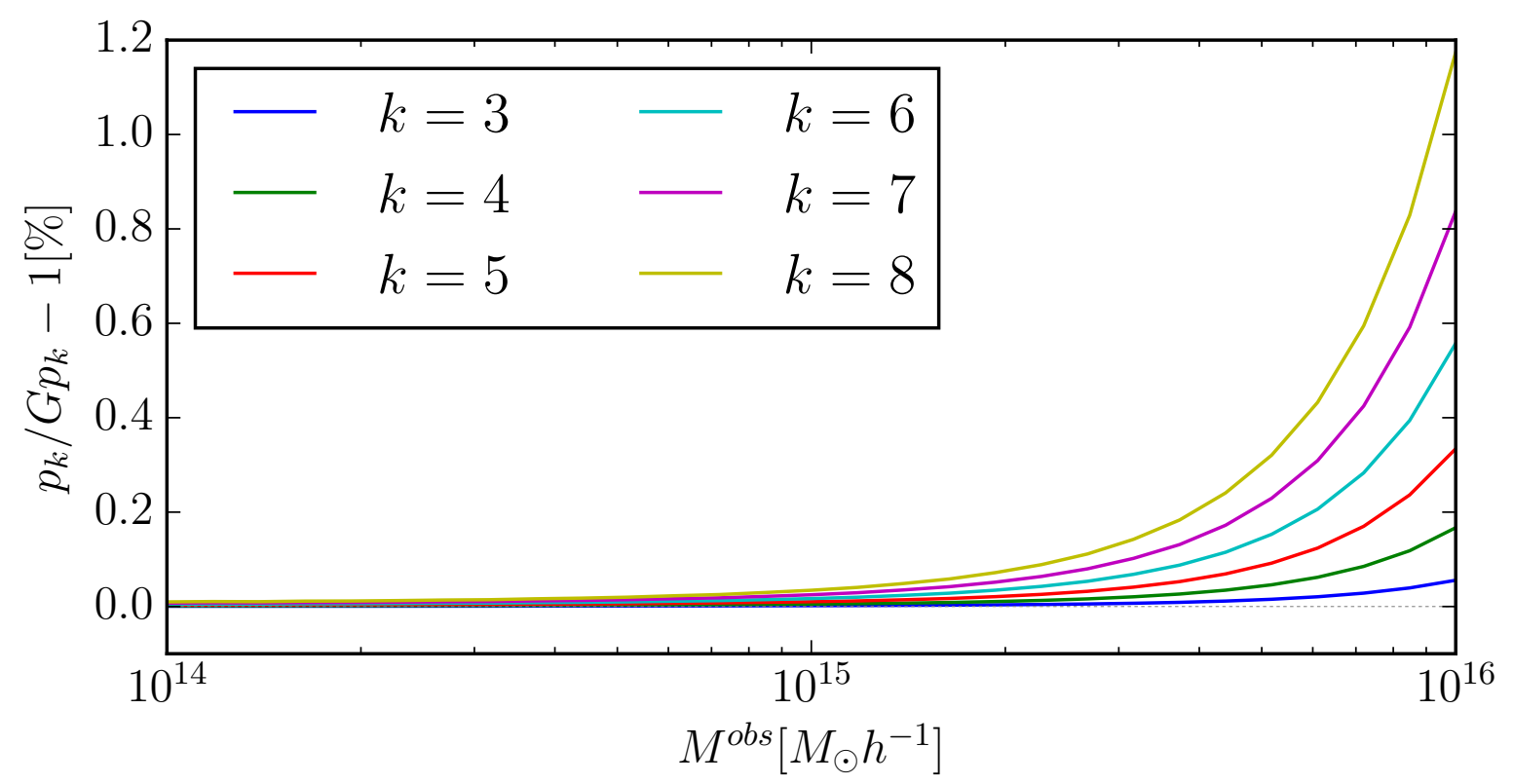

Figure 5.4: Difference between the measured moments $p_{k}$ of $P\left(M \mid M^{\text {obs }}\right)$ and the Gaussian moments $G p_{k}$ computed from the first two moments $p_{1}$ and $p_{2}$.

\subsection{Cosmological Parameters from Dark Matter Halos}

The prediction of galaxy cluster abundance incorporates observational effects from several sources and can quickly become quite complex. Thus, it is easier to introduce each correction independently and examine the effects individually.

Here we explore the power of using dark matter halos to constrain different cosmological parameters. Using the Fisher Matrix formalism we will analyze the effects of fixing, marginalizing and adding priors to the different parameters as well as different redshift ranges. The fiducial case adopted is the same as in section 2.4, computed with a threshold mass of $10^{13.8} M_{\odot} h^{-1}$ in $\mathrm{a} \approx 10,313 \mathrm{deg}^{2}$ area, using Tinker mass function (section 2.3.4 and the fiducial cosmology: $\left(\Omega_{m}=0.27, w=-1, \sigma_{8}=0.88, h=0.7, n_{s}=0.96, \Omega_{b}=0.04\right)$.

For didactic purposes, we will start with a very basic analysis and study additional steps adding them one by one. Firstly, we will consider only the halo abundance information to 
probe cosmological parameters and study the constraints of two parameters at a time, fixing all others. Then, we explore constraining all parameters together, where constraints for each pair of parameters results from marginalization over the other parameters. Next, we study the inclusion of priors on parameters. Finally, we investigate the use of the halo covariance for constraining cosmology as well.

\section{Constraints from pairs of parameters}

Understanding the correlations of constrained parameters is important to determine how we can improve observations to produce the best constraints. When multiple parameters are constrained at the same time, the correlation between each pair of parameters may also depend on the correlation with the other parameters. Therefore, it may be difficult to have a reasonable intuition of this correlation simply from how each parameter affects the halo abundance as discussed in section 2.4. In this section we start by obtaining constraints on only two parameters, where there is no hidden correlation and the conclusions of section 2.4 should be valid.

Using only the abundance of halos to constrain parameters, i.e. fixing the covariance matrix and using only the first term of Eq. 4.19, we will evaluate the efficiency of the redshift depth. Because the behavior of the halo number counts under variation of cosmological parameters (section 2.4) differs from low to high redshifts, we will split the analysis into three cases: considering halos of lower redshifts only $(0<z<1.0)$, restraining to higher redshift $(1.0<z<2.0)$ halos, and using all halos. The $68 \%$ constraints for all combinations of parameters estimations are shown in Fig. 5.5. The blue filled area is the constraints using all halos, the green line is for only lower redshifts and red for higher redshifts. Note that, each panel is independent of each other, since they present the constraints for only a pair or parameters at a time with all other fixed. 


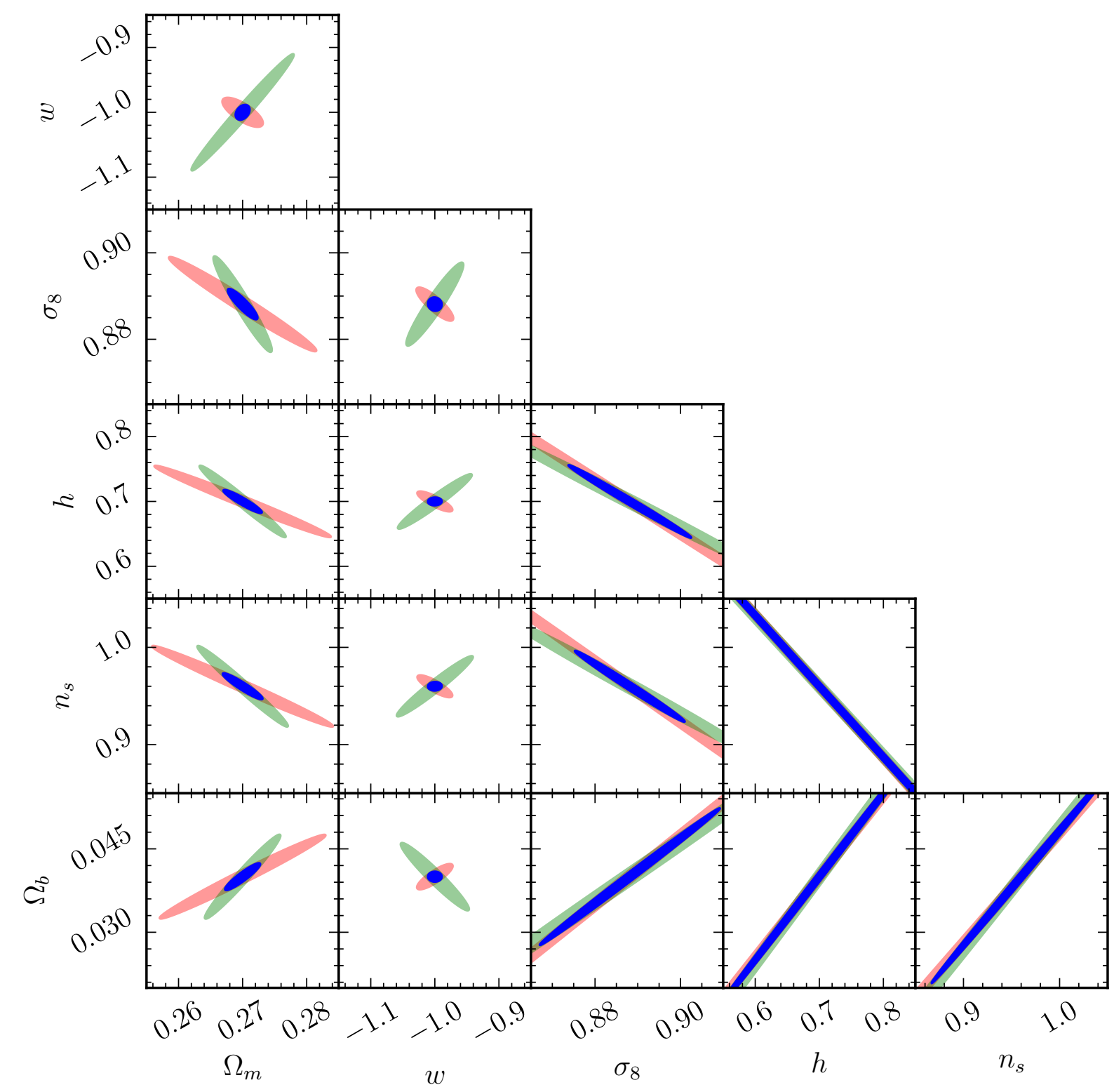

Figure 5.5: Constraints on cosmological parameters using the abundance of halos only for different redshift ranges. The blue solid area are using all halos, in green only the lower redshift halos $(z<1.0)$ and in red only the higher redshift halos $(z>1.0)$. For each panel only the parameters shown were varied, fixing all other parameters.

In the top panel of Fig. 5.5 we have constraints for $\Omega_{m}$ and $w$, where the use of all halos in this very idealistic approach results in extremely good constrains $\left(\sigma_{\Omega_{m}}, \sigma_{w}\right)=(0.0075,0.00071)$. However, if only the low redshifts are used, there is a large degradation of $(685 \%, 635 \%)$ on $\left(\sigma_{\Omega_{m}}, \sigma_{w}\right)$. Meanwhile, using only the high redshift halos, the degradation is much smaller (96\%,191\%), though significant. Another interesting feature is the correlation between these parameters. As shown in section 2.4, an increase in $\Omega_{m}$ and $w$ leads to opposite results on the abundance of halos at low redshifts. This causes a positive correlation between these param- 
eters, i.e. a higher value of $\Omega_{m}$ leads to a higher value for $w$. For high redshifts we have that the opposite effect, an increment on both parameters results in a higher halo counts. Thus the correlation is negative at this region. Even though the higher redshift halos alone constrain well $\Omega_{m}$ and $w$, the lower redshift correlation is very important when using all halos.

In all pairs that do not include $w$, the area of green ellipses is always smaller than of red ellipses. This happens because the peak of the halo distribution is located at the lower redshift range $\left(z_{\text {peak }} \approx 0.5<1.0\right)$ and fitting the shape and position of the peak is much more restraining. However, as seen in Fig. 2.2, the effect of $w$ variations on the halo number counts at low redshift is opposite to the effect at high redshifts, resulting in correlations of $w$ with inverse signals. Because the transition of $w$ effects on the halo abundance is located at our higher redshift case $\left(z_{t r}>1.0\right)$, this region has two ranges $\left(1.0<z<z_{t r}\right.$ and $\left.z>z_{t r}\right)$ with inverse correlations between $w$ and the other parameters, providing better constraints for $w$.

Here we also have a possible indication that some pairs of cosmological parameters (ex. $\left.h-\sigma_{8}, n_{s}-h, \Omega_{b}-h, \ldots\right)$ may not be constrained by the abundance of clusters alone, being almost completely degenerate. Fortunately, some of these parameters are very well constrained by $\mathrm{CMB}$ and other probes.

\section{Constraints using all parameters}

The constraints on Fig. 5.5 are useful to understand the correlations between pairs of parameters. However, this case is too simple and idealistic as it requires perfect knowledge of all other parameters. This assumption not only improves the constraints themselves, but also simplifies correlations among parameters. In Fig. 5.6 we computed the joint constraints for all parameter without any prior knowledge. Each panel has the $68 \%$ and $95 \%$ confidence regions marginalizing over the other parameters.

The constraints for all parameters were severely worse than the previous case, being 1-4 orders of magnitude larger. This resulted in ellipses covering regions with values that have no actual physical meaning for all parameters. Additionally, all correlations were magnified and the parameters are quite degenerate.

\section{Using priors}

We can improve the constraints by considering priors on the cosmological parameters. These priors can be provided by complementary cosmological probes, or simply to exclude values 


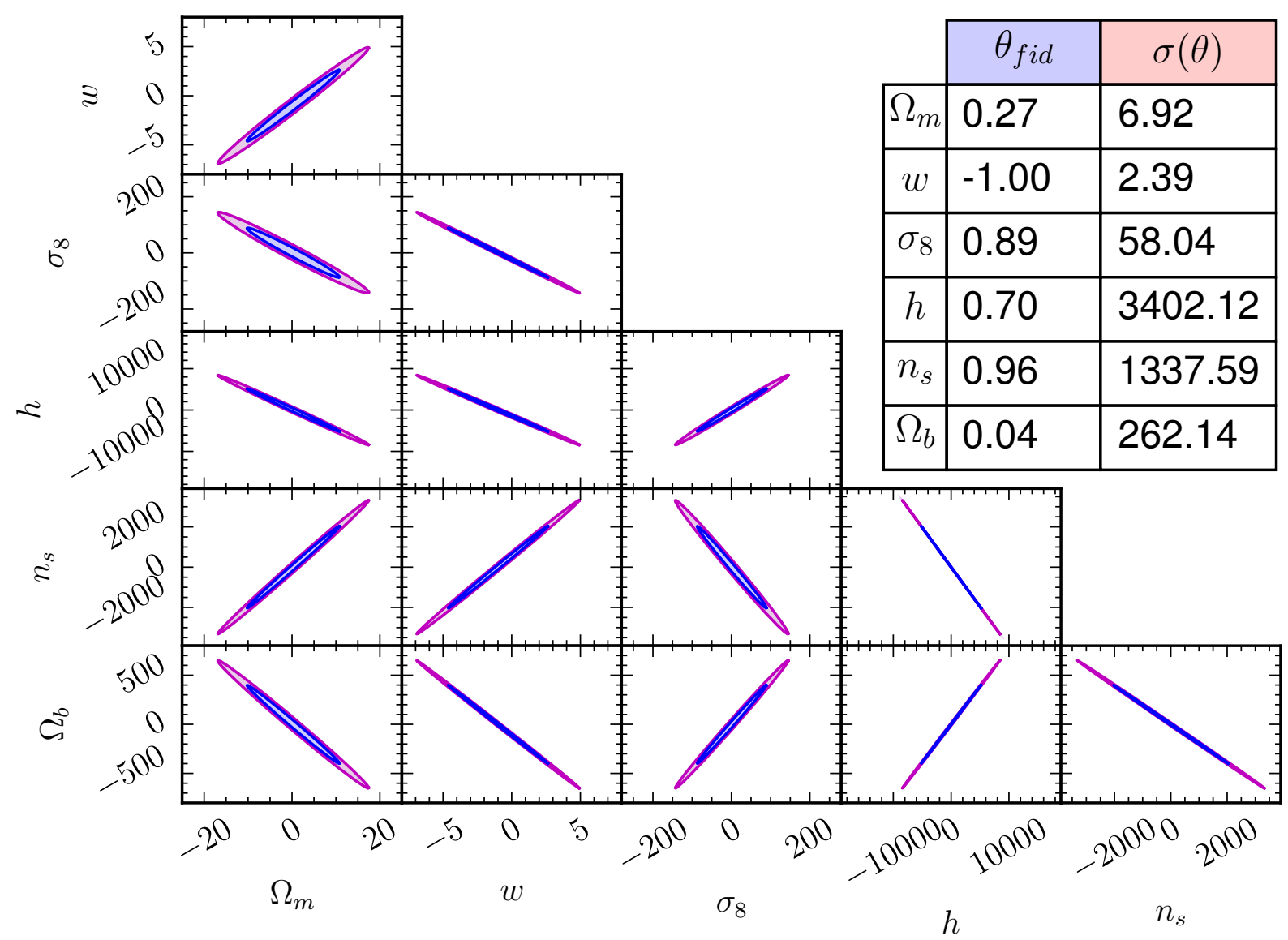

Figure 5.6: Constraints on cosmological parameters using the abundance of halos without any prior knowledge.

without physical meaning. By adding a very loose prior of $\sigma_{p}=1.0$ to each parameter we have a great improvement on the constraints, shown in Fig. 5.7

The degeneracy was reduced for all pairs of parameters, also changing the signal in several cases. For $h$ and $n_{s}$, the resulting constraints were practically the value of the prior introduced. Therefore, for these parameters, the abundance of halos provided effectively no constraints. For $\Omega_{b}$ and $\sigma_{8}$ the prior had some influence but was not dominant, resulting in constraints smaller but of the same order. The greatest improvement were on $\Omega_{m}$ and $w$ with constraints much smaller than the priors. In those parameters, their direct priors had no effect, but the priors on the other parameters provided a great improvement via the correlations.

\section{Constraints from Halo Covariance}

Finally, we consider using the covariance as well as the abundance of halos to recover the cosmological information. In Fig. 5.8, the cosmology was constrained using the complete Fisher 


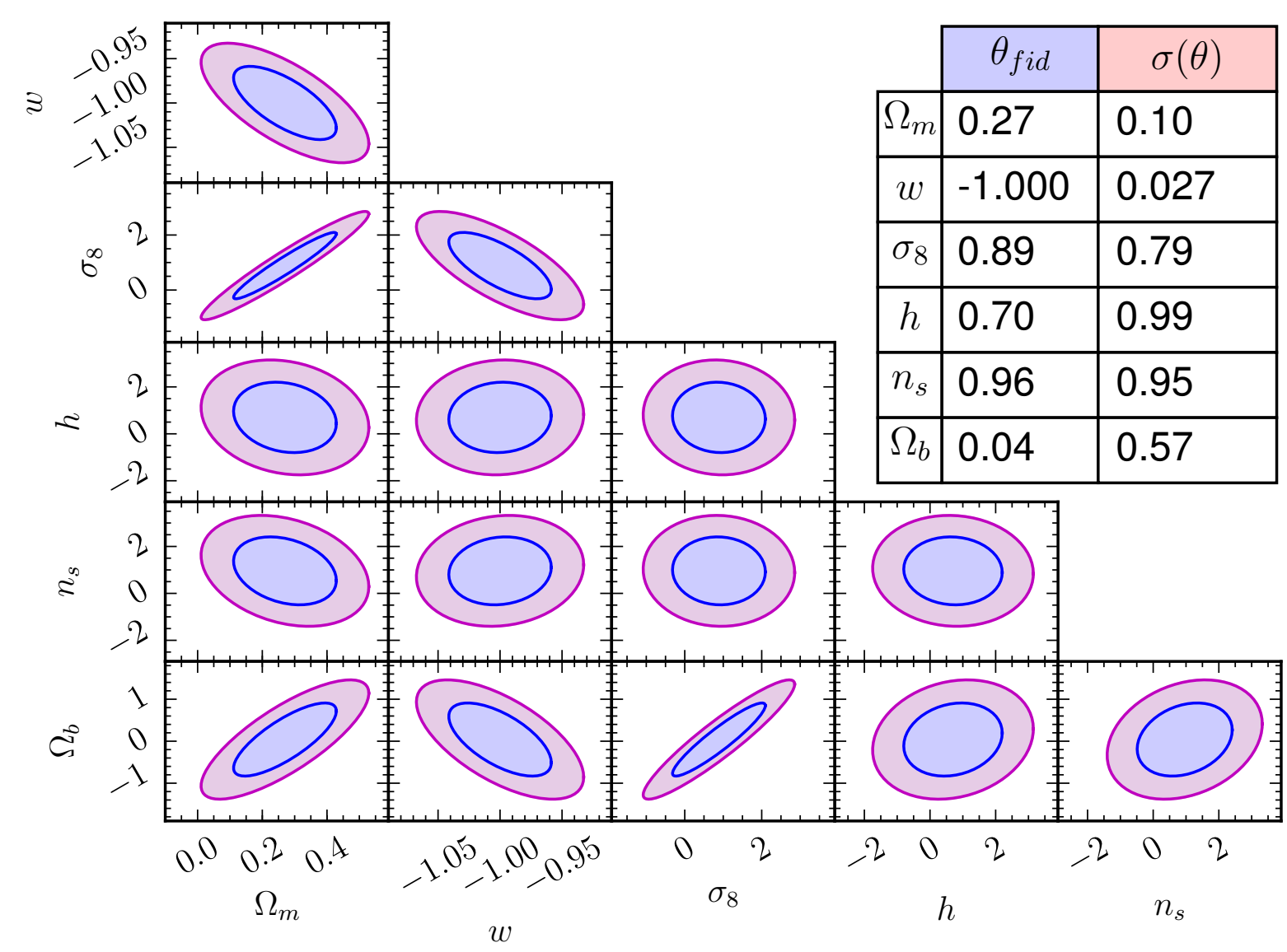

Figure 5.7: Constraints of cosmological using the abundance of halos with Gaussian priors of $\sigma_{\theta}=1.0$.

Matrix from Eq. 4.19, without any prior knowledge.

All constraints are greatly improved compared to the case without use of the covariance matrix information and even compared to the case of only abundance information and loose priors $\sigma_{p}=1.0$. In fact when priors of $\sigma_{p}=1.0$ are added, these results keep nearly unchanged.

The dark energy equation of state parameter $w$ improved by 37\%, while all other parameters had an improvement of over $96 \%$. This demonstrates the effectiveness of using also the covariance of halos to constraining cosmology. In fact, the covariance brings so much extra information that it can be used to help constrain not only the cosmological parameters, but also to self-calibrate the mass-proxy, photo-z and selection parameters (Lima \& Hu, 2004, 2005, 2007; Aguena \& Lima, 2016). This comes at no extra observational effort, since the covariance can be measured from the cluster data itself. 


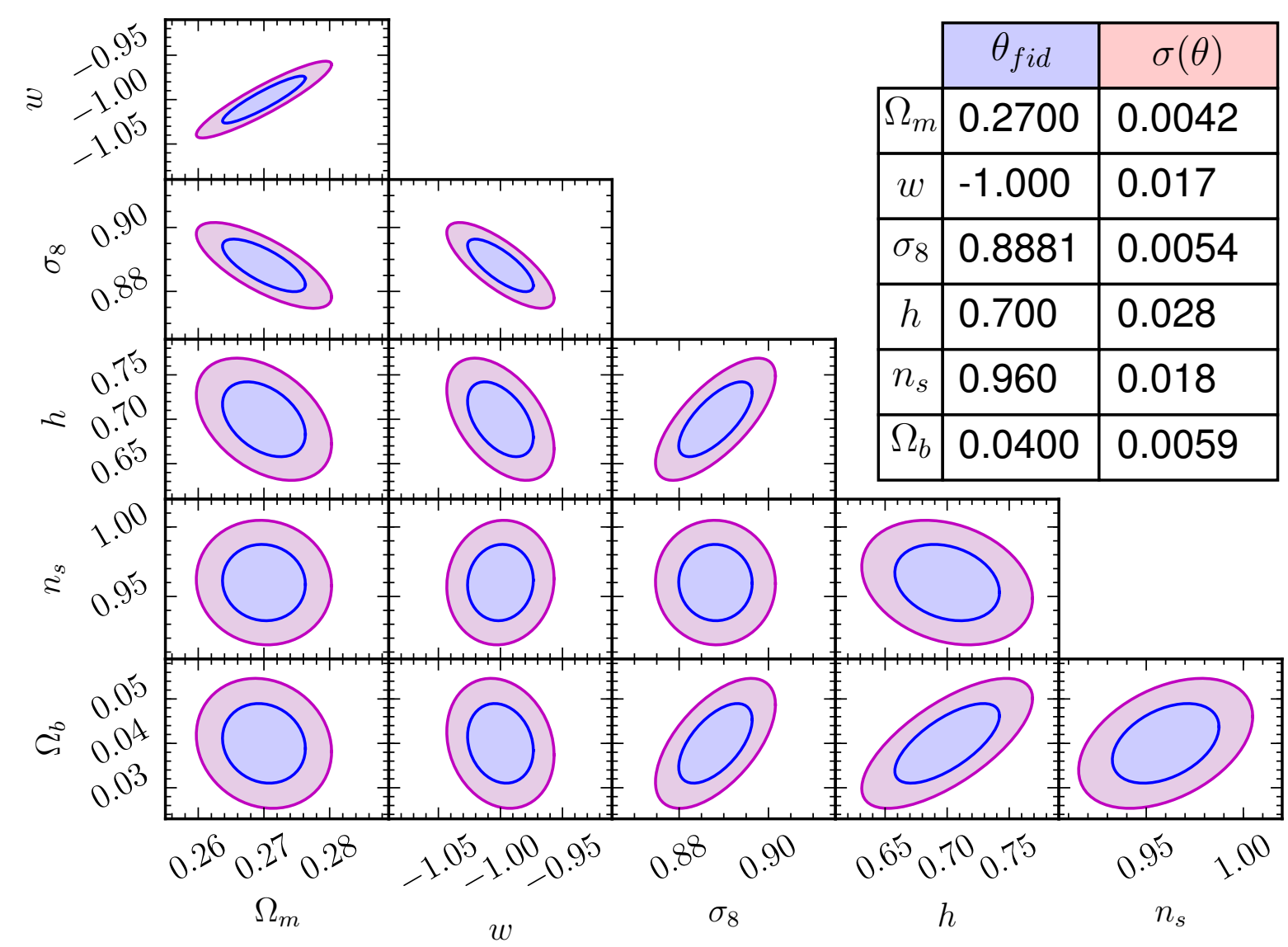

Figure 5.8: Constraints on cosmological parameters using both the abundance and covariance of halos without priors. 


\subsection{Optical Effects on Cluster Dark Energy Constraints}

As discussed in previous sections, the prediction of galaxy cluster abundance may heavily depend on the modeling of the observational effects, which is certainly the case for optical clusters. The mis-characterization or understatement of such effects could lead to significant biases on cosmological constraints. Our goal now is to study how self-calibration of the mass-observable relation and the sample selection function degrade cosmological constraints, in particular on dark energy parameters. We will evaluate how much previous knowledge on these issues can improve the results, as well as having access to wider and deeper surveys. The work from this section was submitted as a scientific paper (Aguena \& Lima, 2016).

The abundance of galaxy clusters is directly related to the mass of the associated dark matter halos, hence characterizing well the relation between the mass of halos and the observable proxy of clusters is imperative to predict cosmology reliably in the cluster framework. This mass-observable relation is usually described not by a perfect function, but by a distribution with a non-negligible scatter, as detailed in sections 3.3 .2 and 5.1. Therefore we will focus on the influence of the parameters that constitute the distribution, specifically the scatter and bias in the context of a Gaussian distribution.

Another important question is the performance of the algorithms for cluster identification (Koester et al., 2007; Rykoff et al., 2014; Soares-Santos et al., 2011; Farrens et al., 2011; Miller et al. 2005; Dietrich et al. 2014). It is possible for cluster finders not only to fail on the detection of the galaxy clusters associated with the dark matter halos, but also to detect "false" clusters, i.e objects with no relation to the dark matter halos. Additionally, over-merging and fragmentation in the detection of galaxy clusters, as well as the quality of photometric redshifts (photo-zs), can affect the measured abundance of clusters. Hence we will also consider the effects of completeness and purity, detailed in section 3.3 .3 .

An extra issue we could consider is redshift errors introduced in the case of photometric surveys (Lima \& Hu, 2007). However, as discussed in 3.3, photo-z errors for cluster galaxies are considerably smaller than for field galaxies. Thus we will neglect this effect in this work, as our goal is to focus on the effects of completeness and purity on dark energy constraints. However, it must be highlighted that, even though we consider the determination of cluster redshifts to be unaffected by the use of photo-zs, it is still possible that the use of photometric redshifts impacts the cluster abundance indirectly via the completeness and purity. 


\subsubsection{Fiducial Model}

We choose a fiducial cosmology from a flat $w$ CDM model with best-fit parameters from the Planck mission (Table 1.1 , We also set priors of $1 \%$ on all parameters, except for the $\Omega_{\mathrm{DE}}$ and $w$, which will vary freely as we wish to study the potential for galaxy clusters to constrain dark energy in the absence of perfect knowledge of the cluster selection function.

For the survey properties, we assumed a similar configuration to that expected for the final observations of the DES, i.e. an angular area of 5,000 $\mathrm{deg}^{2}$ and redshift range with optical limitation of $0.1<z<1.0$. We consider the counts and covariance within 500 angular subdivisions of $10 \mathrm{deg}^{2}$ each. The clusters were divided in nine redshift bins of $\Delta z=0.1$ and seven bins of observed mass with width $\Delta \log \left[M^{\text {obs }} /\left(M_{\odot} h^{-1}\right)\right]=0.2$, from a threshold of $M_{t h}^{\text {obs }}=10^{13.8} M_{\odot} / h$. In order to include all high-mass clusters, the last bin was reshaped to $\log \left[M^{\mathrm{obs}} /\left(M_{\odot} h^{-1}\right)\right]=[15.0: 17.0]$.

The observable-mass $P\left(M^{\mathrm{obs}} \mid M\right)$ distribution will be assumed to be Gaussian in $\ln M^{\text {obs }}$ as parametrized in Eq. 5.2, with a scatter $\sigma_{\ln M^{\mathrm{obs}}}$ and bias $\ln M_{\text {bias }}^{\text {obs }}$. Since we expect a smooth evolution of the mass bias with redshift, we take (Lima \& Hu, 2005)

$$
\ln M_{\text {bias }}^{\mathrm{obs}}(z)=A_{b}+n_{b} \ln (1+z)
$$

where the fiducial values are $A_{n}=n_{b}=0$. Since we expect the mass scatter in the relation to increase for low masses, we take

$$
\frac{\sigma_{\ln M^{\mathrm{obs}}}^{2}(z, M)}{0.2^{2}}=1+B_{0}+B_{z}(1+z)+B_{M}\left(\frac{\ln M_{s}}{\ln M}\right)
$$

with the fiducial values of $B_{0}=B_{z}=B_{M}=0$ and $M_{s}=10^{14.2} M_{\odot} / h$.

The completeness and purity are parametrized as Eqs. 3.13 and 3.14, going from a value of one at high masses to zero at low masses with the sharpness of the transition controlled by parameters $n_{c}$ and $n_{p}$. The actual contribution of these effects is coupled, as described in Eq. 3.18, and the quantity of interest is the ratio of completeness over purity. Hence, there are basically 2 main possible behaviors of $c / p$ to be considered, as exemplified in Table 5.1: case (1) sets values $n_{c}=3$ and $n_{p}=1$, therefore the ratio $c / p$ goes to zero in the limit of low $M$ and $M^{\text {obs }}$; case (2) sets values $n_{c}=1$ and $n_{p}=3$, therefore the ratio $c / p$ goes to infinity in the limit of low $M$ and $M^{\text {obs }}$. These two cases should bracket a reasonable range of possible parametrizations for the selection and their dependence on mass and redshift. 


\begin{tabular}{cccc}
\hline Case & completeness & purity & $c / p($ as $M \rightarrow 0)$ \\
\hline 0 & $c=1$ & $p=1$ & 1 \\
1 & $n_{c}=3$ & $n_{p}=1$ & 0 \\
2 & $n_{c}=1$ & $n_{p}=3$ & $\infty$
\end{tabular}

Table 5.1: Cases considered for completeness and purity parameter values.

For the mass scaling of the completeness and purity we will apply the simple parametrization of Eq. 3.16 with fiducial values of $c_{0}=p_{0}=c_{1}=p_{1}=0$. The values of $\log M_{c f i d}$ and $\log M_{p f i d}^{\text {obs }}$ are arbitrary pivot masses where the completeness and purity decrease to $50 \%$. For illustrative purposes we will fix them to $\log \left[M_{c f i d} /\left(M_{\odot} h^{-1}\right)\right]=13.5$ and $\log \left[M_{p f i d} /\left(M_{\odot} h^{-1}\right)\right]=$ 13.6. This choice of parameters results in a completeness around of $\geq(80 \%, 61 \%)$ and purity $\geq(67 \%, 89 \%)$ around the threshold mass for cases $(1,2)$. In left panel of Fig. 5.9 the completeness and purity for cases $(1,2)$ are displayed. Because completeness is a function of the true mass of dark matter halos, there is a contribution of the completeness from a wide mass range for each value of the cluster observed mass. We illustrate this feature by considering the red line on the purity panel, indicating the fiducial observed mass threshold $\left(10^{13.8} M_{\odot} h^{-1}\right)$. In the completeness panel, this value of observed mass threshold is reflected by the red shaded areas representing the mass scatter at 1,2 and $3 \sigma_{\ln M^{\text {obs }}}$ levels. The effective completeness at the observed threshold mass is a convolution of all values inside the red shaded areas.

In the right panel of Fig. 5.9 the ratio $c / p$ for $M=M^{\text {obs }}$ is shown as well as a $1 \sigma$ deviation in both cases $(1,2)$. In case $(1)$, completeness goes to zero faster than purity as we go to lower masses and the ratio $c / p$ goes to zero. However, in the mass range of interest $\left(M^{\text {obs }} \geq 10^{13.8} M_{\odot} h^{-1}\right.$ ), the ratio is higher than 1, increasing the cluster counts. Case (2) has the inverse behavior of $c / p$ and will result in a lower abundance of clusters.

For reference, we consider an additional case with perfect cluster detection, i.e. completeness and purity equal to unit for all masses and redshifts. We will denote this as case (0) and it will be used to compute the bias induced on dark energy parameters when perfect detection is assumed whereas the true model is either (1) or (2). We will also consider the dark energy constraints derived within models (1) and (2) and prior knowledge in the nuisance parameters describing the observable-mass relation and cluster selection. 



Figure 5.9: Completeness and Purity behavior for cases (1) and (2) at $z=0$. The red vertical line is the threshold mass $M_{t h}^{\text {obs }}=10^{13.8} M_{\odot} / h$ assumed in the fiducial model. Left: Both functions are displayed separated and the red shaded regions (on the top figure) display the mass spread around this threshold at 1,2 and 3 times $\sigma_{\ln M^{\text {obs }}}$. Right: Ratio of completeness over purity as it is used in Eqs. 3.13 and 3.14. Case (1) will result in a increment on the abundance of clusters for higher masses and a deficiency at lower masses, while case (2) has the opposite behavior.

\subsubsection{Cluster Number Counts}

Once we defined the fiducial model, it is useful to investigate the effect of each cosmological and nuisance parameter on cluster abundance, in order to understand the impact on dark energy constraints later on. In Fig. 5.10 we show the sum of cluster number counts in all mass bins as a function of cluster redshift, as predicted for selection parameters of case (1). The thick solid lines are the fiducial model and the colored lines are the abundance with a positive variation in each parameter considered. Based on how the cluster number counts are affected by these variations, we may develop some intuition about the correlation among parameters. A positive correlation between two parameters means that essentially the same cluster abundance can be produced by increasing both parameters simultaneously, hence, the likelihood remains reasonable with the concurring increment on the parameters. In the parameter space, it is reflected by contours with larger preference on the $y \propto x$ axis. Alternatively, when the variation of two parameters have similar influence on the cluster counts, either both increasing or decreasing the abundance, they are anti-correlated. This rationale can provide us with insights on the expected correlations between parameters. However, we must keep in mind that these conclusions are 


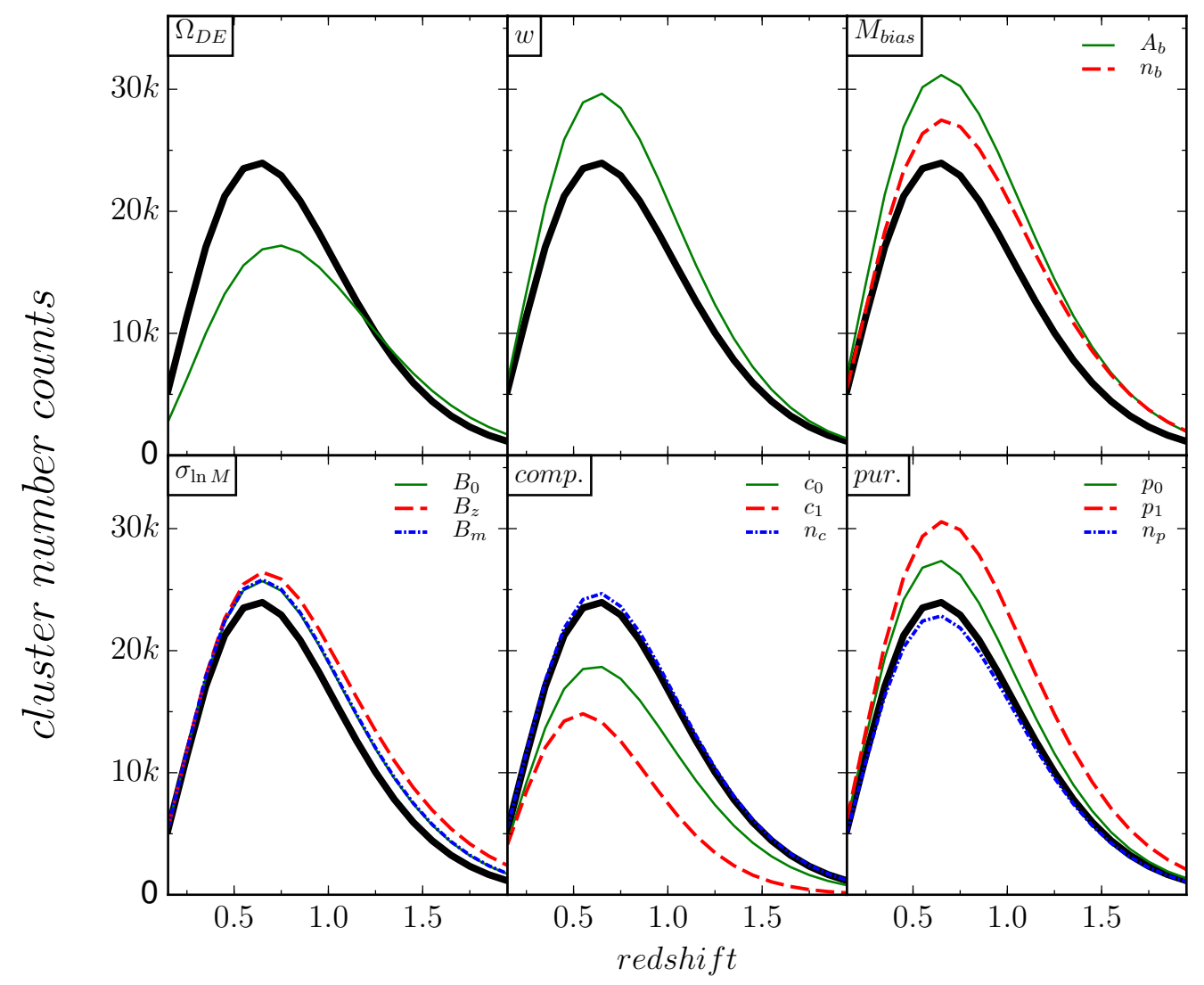

Figure 5.10: Variation of the number counts of the case (1) with respect to the cosmological and nuisance parameters. The black solid line is the fiducial case, the colored lines are a positive variation of 0.2 for each parameter. 
based on variations of only two parameters at a time and rooted on the fiducial values selected. The actual correlations marginalized over all other parameters may be different.

As we fix a flat Universe, an increment in $\Omega_{\mathrm{DE}}$ implies a reduction in $\Omega_{m}$, and the overall abundance of clusters decreases since both effects damp the growth of structure. For $w$, a positive variation means it is less negative (closer to zero and more similar to matter) and dark energy acts less against the gravitational attraction, therefore increasing the cluster counts as well.

When the value of $M_{\text {bias }}^{\text {obs }}$ increases, the effective threshold of mass $M$ is lowered, enhancing the abundance of cluster detected. An akin response happens with an increase on the scatter (Lima \& Hu, 2005), though at a lesser extent, as there is compensation of up and down-scatter objects.

Larger values of $c_{0}$ and $c_{1}$ basically re-scale the completeness value to be valid at a larger mass, lowering the values of completeness across all masses and subsequently the number of clusters detected. Meanwhile, increasing $n_{c}$ leads to a sharper drop in the completeness and the detection becomes more complete for $M>M_{c}$, as it can be seen by comparing cases $(1,2)$ in the left panel of Fig. 5.9. As the purity has the same parametrization as the completeness, the conclusions for the overall purity on the increment of its parameters is the same, resulting in an exact opposite effect on the abundance of clusters detected.

\subsubsection{Selecting Cases}

Before evaluating the effects of completeness and purity on dark energy constraints, we should verify whether it is worth adding this extra level of complexity. By neglecting these selection effects, i.e. by assuming perfect detection (case 0), the precision is enhanced, as fewer parameters have to be constrained. However, a bias $b\left(\Theta_{\alpha}\right)$ (Eq. 4.20 ) is introduced on the recovered parameters. As long as the bias is smaller than the constraint, this approach provides reliable results. This can be verified by computing

$$
b\left(\Theta_{\alpha}\right) \leq \gamma\left(F^{-1}\right)_{\alpha \alpha}^{1 / 2}
$$

where $\gamma=1,2,3$ indicates biased predictions outside the $68 \%, 95 \%$ and $99 \%$ confidence levels. Here the pieces that go into $b\left(\Theta_{\alpha}\right)$ and $F_{\alpha \alpha}^{-1}$ are computed for case (0) and $\Delta \bar{m}$ and $\Delta S$ in Eq. 4.20 are the differences in counts and sample covariance between predictions in case (0) and cases 
$(1,2)$ with completeness and purity.
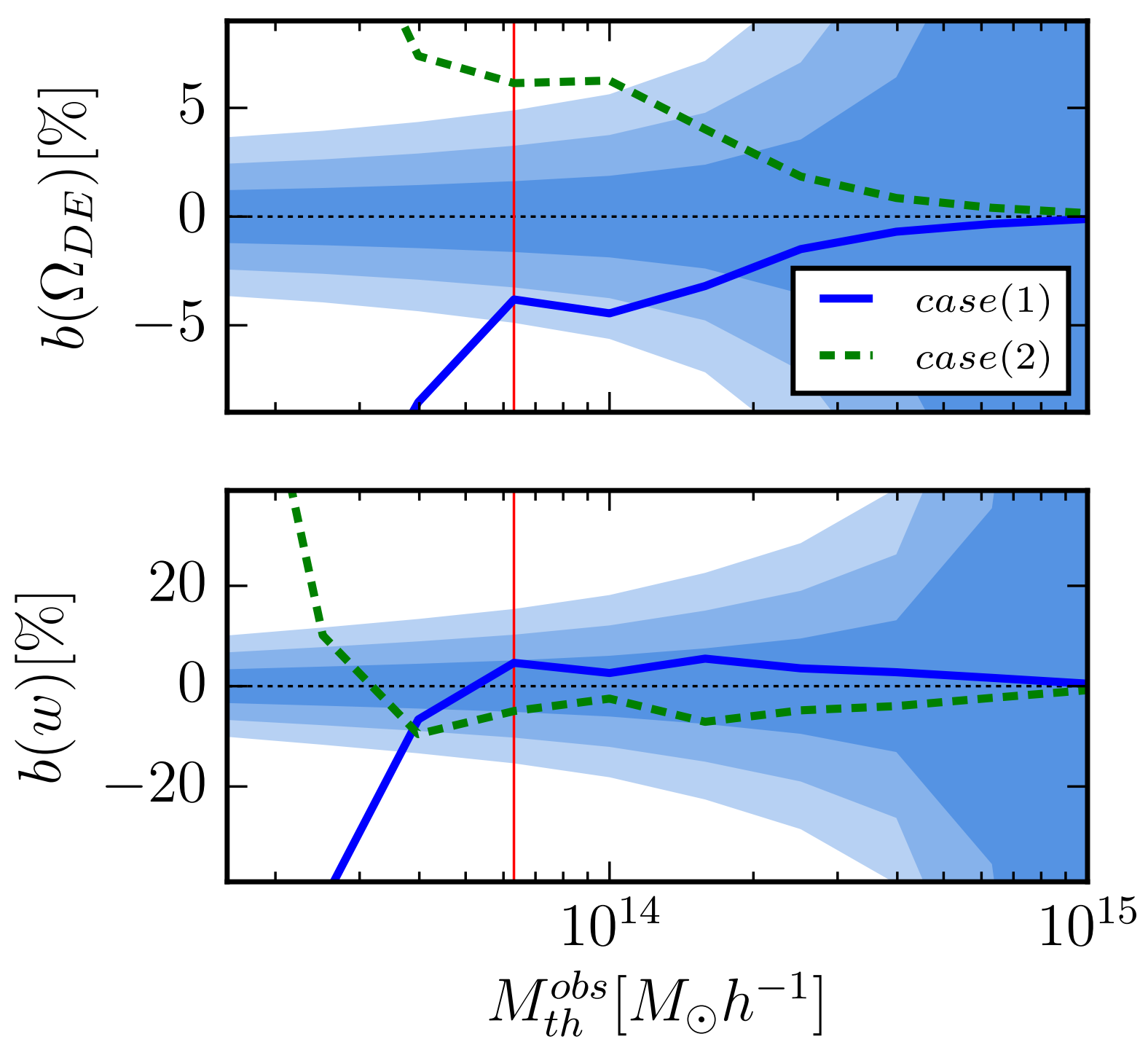

Figure 5.11: Bias $b\left(\Theta_{\alpha}\right)$ on dark energy parameters caused by ignoring completeness and purity effects on different cases. The blue shaded areas are the 68\%, 95\% and $99 \%$ confidence levels assuming perfect cluster detection. The solid, and dashed lines correspond to the cases (1) and (2) respectively (see text). When the bias becomes larger than the $1 \sigma$ constraints $b\left(\Theta_{\alpha}\right)>\sqrt{F_{\alpha \alpha}}$, the assumption of perfect detection results in systematically incorrect cosmological predictions. For the threshold mass considered of $\log \left[M_{t h}^{\text {obs }} /\left(M_{\odot} h^{-1}\right)\right]=13.8$, we have that the bias of $\Omega_{\mathrm{DE}}$ is much larger than $\sigma$ relative to both cases $(1,2)$ and the bias on $w$ is of the same order of $\sigma$.

The bias on dark energy parameters from ignoring completeness and purity for cases (1) and (2) as a function of the observed mass threshold used $M_{t h}^{\text {obs }}$ is shown in Fig. 5.11. We also included the observed mass threshold of our fiducial case (red line) and the $68 \%, 95 \%$ and $99 \%$ confidence levels on the dark energy parameters from case (0) (blue shaded regions). The 

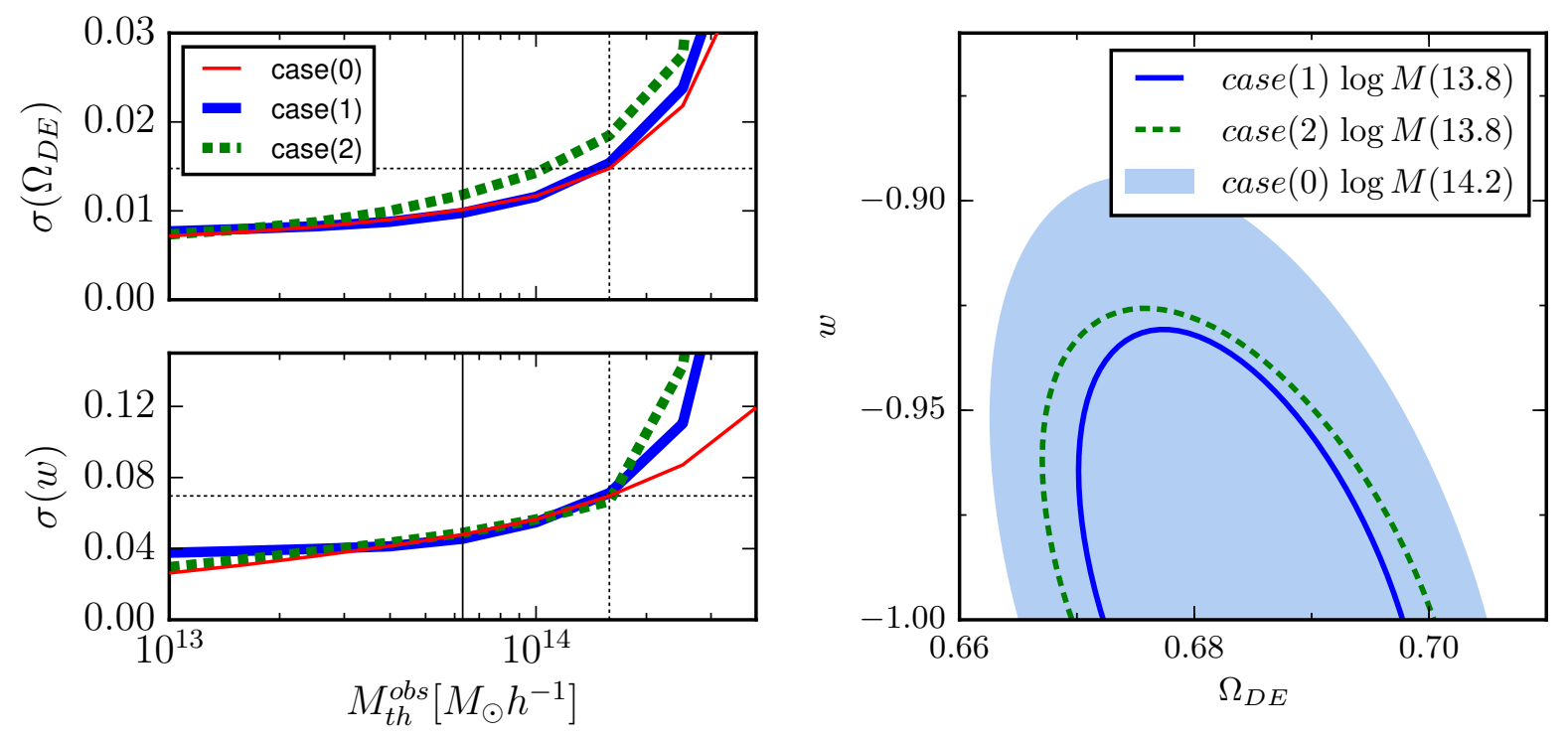

Figure 5.12: Constraints on dark energy parameters for different cases. The shaded area are the constraints of case (0) with the threshold $\log \left[M^{\text {obs }} /\left(M_{\odot} h^{-1}\right)\right]=14.2$, where the bias of $\Omega_{\mathrm{DE}}$


purity with a lower mass threshold $\log \left[M^{\text {obs }} /\left(M_{\odot} h^{-1}\right)\right]=14.2$ actually have better constraints than ignoring these effects and restraining to higher mass thresholds.

bias on $\Omega_{\mathrm{DE}}$ surpasses the $1 \sigma$ constraints at a threshold $\log \left[M_{t h}^{\text {obs }} /\left(M_{\odot} h^{-1}\right)\right]<14.2$ for both cases $(1,2)$, hence the assumption of perfect detection leads to even more biased estimations of $\Omega_{\mathrm{DE}}$ at lower thresholds. In fact, at our fiducial mass threshold, the $\Omega_{\mathrm{DE}}$ estimated is outside the $95 \%$ confidence region for case (1) and $99 \%$ for case (2). For $w$, the bias has a similar amplitude as the $1 \sigma$ errors at $\log \left[M_{t h}^{\text {obs }} /\left(M_{\odot} h^{-1}\right)\right]=13.8$. While this does not necessarily invalidate constraints on $w$ within case $(0)$, it is certainly not a robust result.

On a side note, we investigate the non-monotonic behavior of the bias as a function of threshold masses $M_{t h}^{\text {obs }}$. The main reason is the fact that $c / p$ is also non-monotonic as seen in the right panel of Fig. 5.12. For case (1)/(2), the ratio $c / p$ increases/decreases the cluster counts at higher masses, but decreases/increases them at lower masses, resulting in opposite sign of bias for each mass range. This behavior happens for both $\Omega_{\mathrm{DE}}$ and $w$, however, the inversion of the bias sign for $\Omega_{\mathrm{DE}}$ occurs at lower masses $\left(M^{\mathrm{obs}}<10^{13.2} M_{\odot} h^{-1}\right)$ and is not seen in Fig. 5.11. The convolution of these effects with the correlation among all other parameters in the cluster abundance prediction produce what might appear as an odd bias behavior.

The next step is to consider if, under cases (1) and (2) with our fiducial threshold mass $\log \left[M_{\text {th }}^{\text {obs }} /\left(M_{\odot} h^{-1}\right)\right]=13.8$, the dark energy constraints are an improvement from case $(0)$ with the minimum threshold with reliable results $\log \left[M_{t h}^{\text {obs }} /\left(M_{\odot} h^{-1}\right)\right]=14.2$. The left panel 
of Fig. 5.12 shows $1 \sigma$ constraints for all cases across mass thresholds. Though the constraints from cases $(1,2)$ are always worse than from case $(0)$, the difference decreases as we lower the threshold mass, being $(4 \%, 20 \%)$ for $\Omega_{\mathrm{DE}}$ and $(2 \%, 5 \%)$ for $w$ at $\log \left[M_{t h}^{\text {obs }} /\left(M_{\odot} h^{-1}\right)\right]=14.2$. For the fiducial threshold $\log \left[M_{t h}^{\text {obs }} /\left(M_{\odot} h^{-1}\right)\right]=13.8$, the dark energy constraints for both cases $(1,2)$ represent an improvement of $(51 \%, 25 \%)$ on $\sigma\left(\Omega_{\mathrm{DE}}\right)$ and $(53 \%, 42 \%)$ on $\sigma(w)$ relative to case (0) with $\log \left[M_{t h}^{\text {obs }} /\left(M_{\odot} h^{-1}\right)\right]=14.2$ (black dotted line). The right panel of Fig. 5.12 shows a direct comparison of these cases as the $68 \%$ confidence regions, and the total area of the ellipses for cases $(1,2)$ are $(57 \%, 44 \%)$ smaller than for case $(0)$.

In a nutshell, we can say it is worth including the additional parameters that allow us to lower the mass threshold from $\log \left[M_{t h}^{\text {obs }} /\left(M_{\odot} h^{-1}\right)\right]=14.2$ to $\log \left[M_{t h}^{\text {obs }} /\left(M_{\odot} h^{-1}\right)\right]=13.8$, where completeness and purity are at levels of $60-80 \%$. It must be noted, however, that should one go to even lower threshold masses than the fiducial case, the dark energy constraints would continue to improve indefinitely. This is a limitation of this use of Fisher Matrix forecasts, where we suppose the functional form of the selection effects is valid across all masses. The modeling of observational effects at masses where the completeness and purity are very low become extremely complex and even small errors on the selection in that regime could highly bias the derived constraints. A more conservative approach would be to increase the number of parameters as we go to these lower masses, which would consequently degrade the constraints. Therefore, our constraints at those masses could be used as a guideline, but should not lead to stark conclusions. Despite these limitations, if one can model the completeness and purity down to a trustworthy level where the assumed functional form for the selection is reliable, the additional information from abundance and clustering of clusters can be sufficient to selfcalibrate the extra parameters and provide improvements on dark energy constraints relative to a simpler modelling approach at higher thresholds.

\subsubsection{Completeness and Purity Effects}

In this section we will examine the self-calibrated constraints on the nuisance parameters and how they affect dark energy constraints. To comprehend specifically the influence of the selection function, we will split the observational effects into two categories: observable-mass $(O M)$, previously studied by e.g. Lima \& Hu (2005, 2007), and completeness and purity $(C P)$ Aguena \& Lima (2016). Because the results of both cases $(1,2)$ are very similar and also displayed in Table 5.2, we will only discuss case (1). 


\begin{tabular}{cc|cc|cc}
\hline & & Case (1) & & Case (2) \\
$\Theta_{O M}$ & $\Theta_{C P}$ & $\sigma\left(\Omega_{\mathrm{DE}}\right)$ & $\sigma(w)$ & $\sigma\left(\Omega_{\mathrm{DE}}\right)$ & $\sigma(w)$ \\
\hline \hline fix & fix & 0.006 & 0.033 & 0.006 & 0.036 \\
free & fix & 0.009 & 0.044 & 0.010 & 0.047 \\
fix & free & 0.009 & 0.042 & 0.010 & 0.045 \\
free & free & 0.010 & 0.046 & 0.012 & 0.049 \\
$1 \%$ & free & 0.009 & 0.042 & 0.010 & 0.045 \\
free & $1 \%$ & 0.009 & 0.044 & 0.010 & 0.048 \\
$1 \%$ & $1 \%$ & 0.006 & 0.041 & 0.007 & 0.042
\end{tabular}

Table 5.2: Constraints for dark energy with different scatter and bias $\left(\Theta_{\mathrm{OM}}\right)$, and completeness and purity $\left(\Theta_{\mathrm{CP}}\right)$ considerations.

For the best case scenario, where all parameters of both $O M\left(\Theta_{O M}\right)$ and $C P\left(\Theta_{C P}\right)$ are perfectly known, the dark energy constraints are $\sigma\left(\Omega_{\mathrm{DE}}, w\right)=(0.006,0.033)$. If we fix $\Theta_{C P}$ and let $\Theta_{O M}$ vary freely, these constraints degrade to $(0.009,0.044)$. Alternatively, fixing $\Theta_{O M}$ and letting the $\Theta_{C P}$ free, the constraints become $(0.009,0.042)$, a similar degradation as the previous case. Hence, having self-calibrating either only $\Theta_{O M}$ or only $\Theta_{C P}$ leads to akin dark energy constraints.

In the case where we have no prior knowledge on any observational effect, i.e. both $\Theta_{O M}$ and $\Theta_{C P}$ vary freely, the constraints become $(0.010,0.046)$. This corresponds to a degradation of $(70 \%, 36 \%)$ relative to the case where these functions are perfectly known. Though this is a substantial deterioration, if we compare these constraints to the case with free $\Theta_{O M}$ and fixed $\Theta_{C P}$, the degradation is only $(4 \%, 2 \%)$. Hence, if $\Theta_{O M}$ is being self-calibrated, including $\Theta_{C P}$ is tremendously advantageous, as it covers a larger array of cluster detection issues without degrading dark energy constraints considerably.

Applying priors of $1 \%$ (or $10^{-2}$ when the fiducial value is zero) on $\Theta_{O M}$ but letting $\Theta_{C P}$ free the constraints become $(0.009,0.042)$. Conversely, if we let $\Theta_{O M}$ vary freely and apply a $1 \%$ prior on $\Theta_{C P}$, the constraints become $(0.009,0.044)$. Once again the influence of the knowledge on either $\Theta_{O M}$ or $\Theta_{C P}$ on dark energy constraints is almost identical.

External priors may come either from simulations, lensing masses for a cluster sub-sample or cross-calibrations with x-ray or SZ detections. In all cases, they are likely to provide clues on the correct functional forms for these functions and conservative ranges for both the richnessmass relation and the completeness/purity parameters. Applying therefore a 1\% prior to all these parameters from external calibrations, the constraints become $(0.006,0.041)$. This corresponds to a degradation of $(8 \%, 21 \%)$ relative to the case in which these nuisance parameters are 

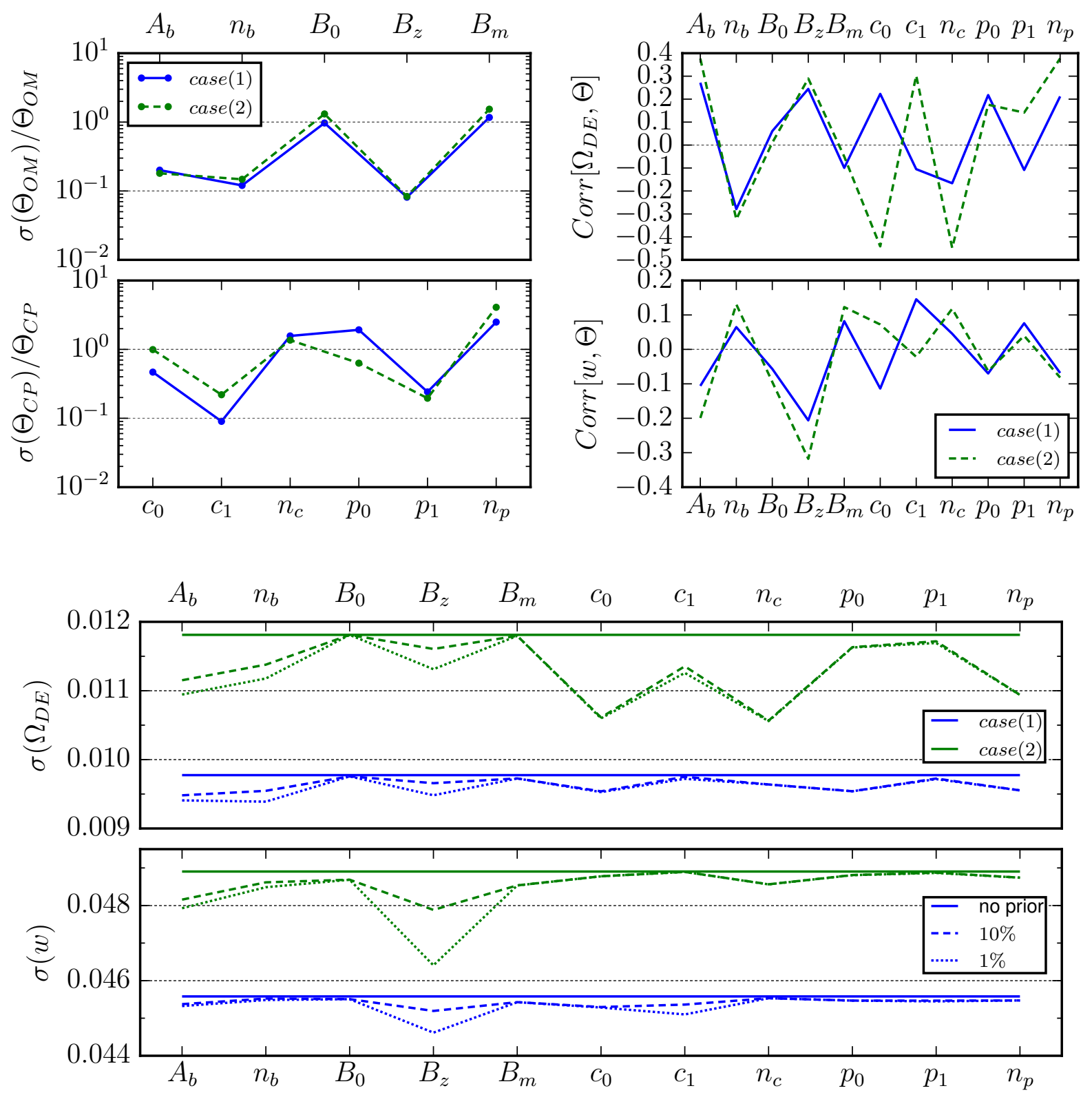

Figure 5.13: Constraints from the Fisher Matrix approach on observational effects. In the left are the constraints derived for nuisance parameters in case (1) (blue solid line) and case (2) (green dashed line). In the right are the constraints for $\Omega_{\mathrm{DE}}$ and $w$ resulting by adding individual prior in each nuisance parameter. The parameters are related to the observable-mass relation (top panel) and completeness/purity (bottom panel). No prior knowledge was assumed for these nuisance parameters. 
perfectly known.

In order to better understand the improvements on dark energy constraints from priors on nuisance parameters, we investigate constraints on nuisance parameter themselves from selfcalibration. On the top left panel of Fig. 5.13, these constraints are displayed normalized by the fiducial value (or by 1 when the fiducial is null). About half of the parameters $\left(B_{0}, B_{m}, c_{0}\right.$, $n_{c}, p_{0}$ and $\left.n_{p}\right)$ are poorly constrained, with $\sigma\left(\Theta_{\alpha}\right) / \Theta_{\alpha}^{\text {fid }}$ around $100 \%$. For these parameters, a prior of $1 \%$ represents a significant improvement and could result in better dark energy constraints. However, the real improvement on the constraints will also depend on the correlation $\left(F_{\alpha \beta} / \sqrt{F_{\alpha \alpha} F_{\beta \beta}}\right)$ between nuisance and dark energy parameters (top right panel of Fig. 5.13.

In the bottom panel of Fig. 5.13, we show the constraints on dark energy with priors of $10 \%$ and $1 \%$ applied individually on each nuisance parameter. In this figure we can see clearly how improvements on each nuisance parameter affects the dark energy constraints. When we set the prior value at $10 \%$, the best improvement on $\Omega_{\mathrm{DE}} / w$ for case (1) occurs when it is applied to $A_{b} / B_{z}$, even though the worst constrained parameter is $n_{p}$. It happens as the absolute value of the correlation between $\Omega_{\mathrm{DE}}-A_{b} / w-B_{z}$ is higher than $\Omega_{\mathrm{DE}}-n_{p} / w-n_{p}$. Additionally, we can note that the absolute value of the correlation $\Omega_{\mathrm{DE}}-n_{b}$ is higher than $\Omega_{\mathrm{DE}}-A_{b}$, but a $10 \%$ prior on $A_{b}$ still results in better improvements in $\Omega_{\mathrm{DE}}$ constrains than a $10 \%$ prior on $n_{b}$. In this case, the value of the self-calibrated constraint $\left(\sigma\left(A_{b}\right) / A_{b}>\sigma\left(n_{b}\right) / n_{b}\right)$ was more influential than the correlation between parameters. However, setting the prior at $1 \%$, a value less or of the order of all nuisance parameters, the improvements on the dark matter parameters are dictated solely by the correlation between each nuisance dark energy parameter.

Finally, in order to quantify the effects of priors $\sigma_{\mathrm{p}}\left(\Theta_{\mathrm{n}}\right)$ assumed on multiple nuisance parameters $\Theta_{\mathrm{n}}=\left(\Theta_{\mathrm{OM}}, \Theta_{\mathrm{CP}}\right)$, we define the degradation factor $\mathcal{D}_{\Theta_{\mathrm{DE}}}$ on the constraints of dark energy parameters $\Theta_{\mathrm{DE}}=\left(\Omega_{\mathrm{DE}}, w\right)$ as

$$
\mathcal{D}_{\Theta_{\mathrm{DE}}}\left[\sigma_{p}\left(\Theta_{\mathrm{n}}\right)\right]=\frac{\sigma\left[\Theta_{\mathrm{DE}} \mid \sigma_{p}\left(\Theta_{\mathrm{OM}}\right), \sigma_{p}\left(\Theta_{\mathrm{CP}}\right)\right]}{\left.\sigma\left(\Theta_{\mathrm{DE}}\right)\right|_{\mathrm{ref}}}-1 .
$$

This factor represents the relative difference between constraints on $\Theta_{\mathrm{DE}}$ given priors $\sigma_{\mathrm{p}}\left(\Theta_{O M}\right)$ and $\sigma_{\mathrm{p}}\left(\Theta_{C P}\right)$ and the reference ideal case $\left.\sigma\left(\Theta_{\mathrm{DE}}\right)\right|_{\text {ref }}=\sigma\left[\Theta_{\mathrm{DE}} \mid 0,0\right]$ where nuisance parameters are perfectly known. In order to analyze the effects of priors on nuisance parameters, we will consider these parameters separately by categories $O M$ and $C P$.

In Fig. 5.14 we show contours of fixed degradation on dark energy constraints for different priors on $\Theta_{O M}$ and $\Theta_{C P}$. As both panels of Fig. 5.14 present a very similar behavior, let us take 

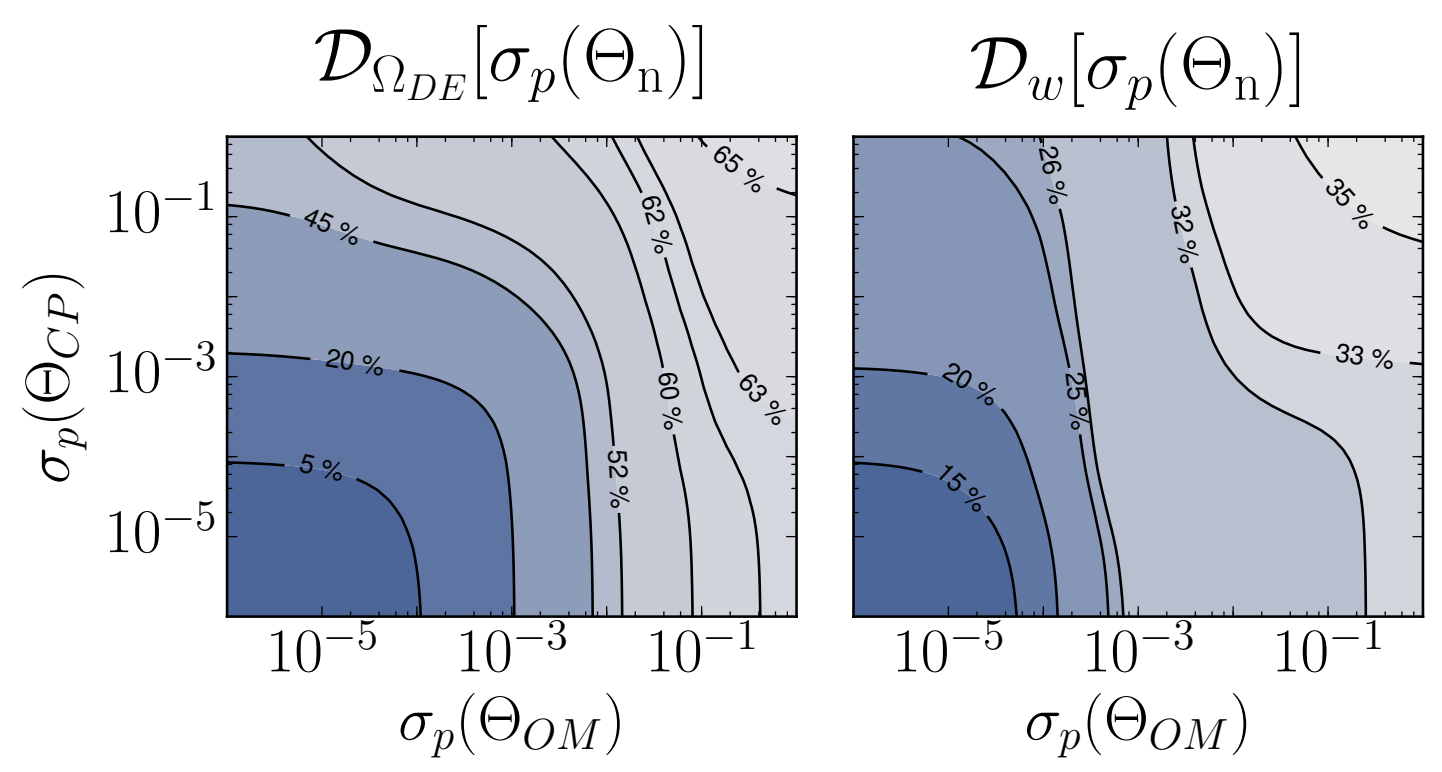

Figure 5.14: Degradation on dark energy constraints for case (1) with priors on the nuisance paremeters relative to the case where these parameters are fixed (Eq. 5.13). For a given value of $\sigma_{\mathrm{p}}\left(\Theta_{O M}\right)$, the same prior is applied on all the richness-mass relation parameters. The same happens for $\sigma_{\mathrm{p}}\left(\Theta_{C P}\right)$ and the completeness/purity parameters.

consider only $\sigma\left(\Omega_{\mathrm{DE}}\right)$. For a significant improvement on dark energy constraints, it is necessary to have knowledge of both $\Theta_{O M}$ and $\Theta_{C P}$. If $\sigma_{\mathrm{p}}\left(\Theta_{C P}\right) \geq 0.1$, degradation cannot be lower than $60 \%$. Conversely, having $\sigma_{\mathrm{p}}\left(\Theta_{O M}\right) \geq 0.1$ limits the minimum degradation to $45 \%$. In order to keep the degradation lower than $20 \%$, we need both $\sigma_{\mathrm{p}}\left(\Theta_{O M}\right)$ and $\sigma_{\mathrm{p}}\left(\Theta_{C P}\right)$ at a sub-percent level, which may be quite challenging.

\subsubsection{Future Surveys}

The conclusions of this chapter were based on a configuration similar to expectations of the DES, representing current surveys. For future surveys (Predehl et al., 2010; Amendola et al., 2013; Tyson, 2002), we expect major improvements on both total angular area and depth, i.e. maximum redshift. In this section we will investigate constraint improvements and $C P$ significance in these future scenarios.

In our approach of splitting the survey into equal parts of $10 \mathrm{deg}^{2}$, the Fisher Matrix has a linear relation with total area. Consequently, the constraints for all parameters scale with the square root of the area. Our fiducial area of 5,000 deg ${ }^{2}$ is similar to what will be observed by the DES after 5 years of observations. An area twice as large (1/4th of sky) would result 


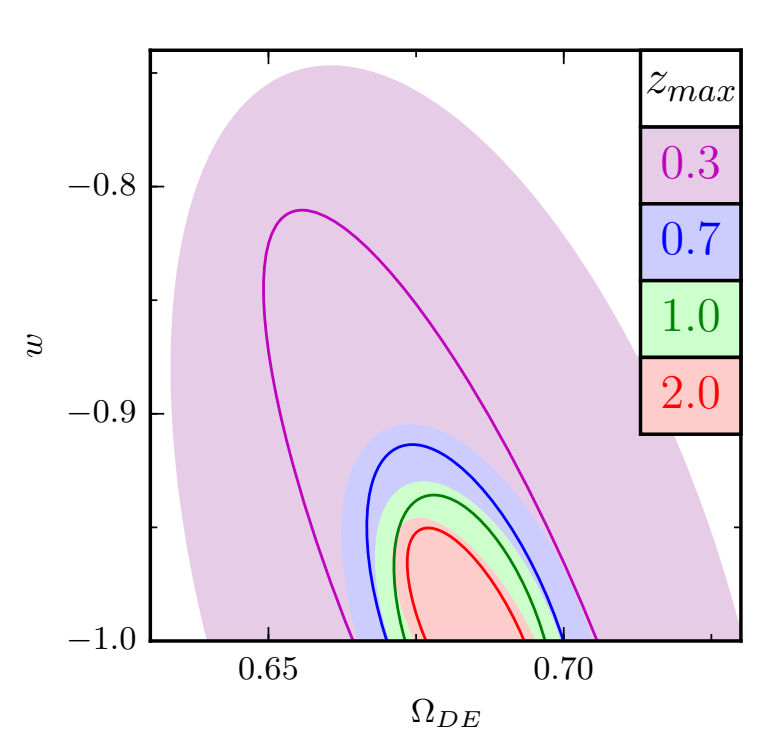

Figure 5.15: Dark energy constraints considering different values of maximum redshift $z_{\max }$.

\begin{tabular}{c|cc|cc}
\hline & Case (1) & & Case (2) & \\
$z_{\max }$ & $\sigma\left(\Omega_{\mathrm{DE}}\right)$ & $\sigma(w)$ & $\sigma\left(\Omega_{\mathrm{DE}}\right)$ & $\sigma(w)$ \\
\hline \hline 0.3 & 0.033 & 0.201 & 0.051 & 0.254 \\
0.5 & 0.018 & 0.089 & 0.025 & 0.100 \\
0.7 & 0.014 & 0.068 & 0.017 & 0.077 \\
1.0 & 0.010 & 0.046 & 0.012 & 0.049 \\
1.2 & 0.009 & 0.040 & 0.010 & 0.044 \\
1.5 & 0.008 & 0.035 & 0.010 & 0.039 \\
1.7 & 0.008 & 0.034 & 0.009 & 0.037 \\
2.0 & 0.008 & 0.033 & 0.009 & 0.035
\end{tabular}

Table 5.3: Constraints for dark energy as a function of maximum redshift $z_{\max }$. Here all nuisance parameters describing the effective selection function (observable-mass, completeness and purity) vary freely.

in an improvement of $\approx 29 \%$ on both dark energy parameters. If half the sky is observed the constraints improve by $\approx 50 \%$ and the best improvement possible would be $\approx 65 \%$ by using the full-sky.

The impact of survey depth on dark energy constraints is displayed in Fig. 5.15. This limitation in redshift may come not only from survey observations, but from the cluster finder itself. Considering the history of cluster cosmology, the MaxBCG cluster sample based on SDSS data (Koester et al., 2007) had a limitation of $z_{\max }=0.3$. Within our analysis, this case results on $\sigma\left(\Omega_{\mathrm{DE}}, w\right)=(0.033,0.201)$, a deterioration of $(235 \%, 341 \%)$ relative to our fiducial case $\left(z_{\max }=1.0\right)$. The more recent redMaPPer cluster finder (Rykoff et al., 2014) produced catalogs with redshifts up to $z_{\max }=0.7$, corresponding to constraints of $(0.014,0.068)$, a degradation of $(43 \%, 48 \%)$ relative to our results. For upcoming surveys going to higher redshifts, we find constraints of $(0.008,0.033)$ for $z_{\max }=2$, an improvement of $(22 \%, 28 \%)$. A more complete description of dark energy constraints across different values of $z_{\max }$ is shown in Table 5.3. Case (2) presents a higher degradation when lowering $z_{\max }$ than case (1), and a lower improvement when we extend $z_{\max }$.

To quantify the completeness and purity effects across different redshift depths, we will compute the degradation $\mathcal{D}_{\Theta_{\mathrm{DE}}}$ of dark energy constraints for the cases with free completeness and purity parameters $\sigma\left[\Theta_{D E} \mid \sigma_{p}\left(\Theta_{C P}\right)=\infty\right]$ relative to the case with perfect knowledge 

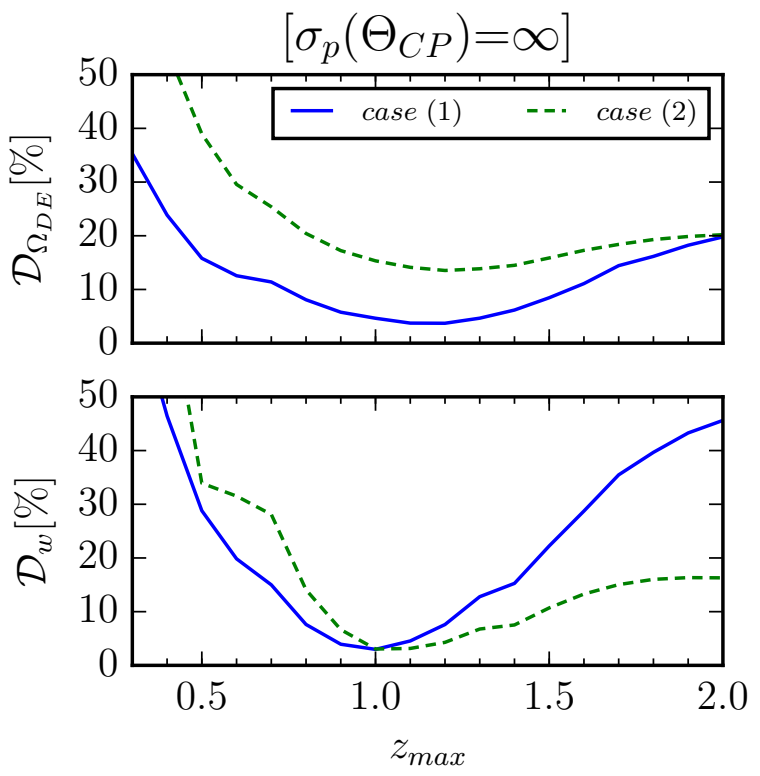



Figure 5.16: Effects of completeness and purity as a function of maximum redshift $z_{\max }$. On the left is shown the degradation of dark energy constraints for the case where completeness and purity parameters are free $\left(\sigma_{p}\left(\Theta_{C P}\right)=\infty\right)$ relative to the case where these parameters are fixed $\left(\sigma_{p}\left(\Theta_{C P}\right)=0\right)$. On the right are shown the actual dark energy constraints in both cases.

$\sigma\left[\Theta_{D E} \mid \sigma_{p}\left(\Theta_{C P}\right)=0\right]$. In the left panel of Fig. 5.16, we see that $\mathcal{D}_{\Theta_{D E}}$ has a significant overall improvement (i.e. it decreases) with the increase of $z_{\max }$, up to $z_{\max } \approx 1.0-1.2$. At higher redshifts, $\mathcal{D}_{\Theta_{D E}}$ rises again. Note that this increase of degradation does not necessarily mean that the dark energy constraints worsen as we include higher redshifts, but the effects of completeness and purity become more significant. The actual values of dark energy constraints are shown on the right panel of Fig. 5.16, We see that the improvement of $\mathcal{D}_{\Theta_{D E}}$ at lower maximum redshifts $\left(z_{\max } \lesssim 1.0-1.2\right)$ happens as $\sigma\left[\Theta_{D E} \mid \sigma_{p}\left(\Theta_{C P}\right)=\infty\right]$ has a sharper decline than $\sigma\left[\Theta_{D E} \mid \sigma_{p}\left(\Theta_{C P}\right)=0\right]$ in that region. However, by including higher redshifts, $\sigma\left[\Theta_{D E} \mid \sigma_{p}\left(\Theta_{C P}\right)=\infty\right]$ has a much flatter decrease than $\sigma\left[\Theta_{D E} \mid \sigma_{p}\left(\Theta_{C P}\right)=0\right]$ and $\mathcal{D}_{\Theta_{D E}}$ starts rising.

Ultimately, we find that the degradation from the effects of completeness and purity become more relevant as the depth increases, specially for $w$ on case (1). As we probe higher redshifts, we gain more information on the history of expansion of the universe and consequently on $w$. Thus, the degradation from uncertainties introduced by the selection function can also become more significant. 


\section{Chapter 6}

\section{Simulation Results}

Simulations are extremely useful to understand both the properties of dark matter halos and the performance of cluster finders, as it is further discussed in Appendix A. In this chapter, we will analyze the MICE simulation (Fosalba et al., 2015b; Crocce et al., 2015; Fosalba et al., 2015a) and a DES simulation. Both these simulations are dark matter N-body simulations, covering wide areas and of high depths. Additionally, these simulations provide dark matter halo and galaxy catalogs constructed based on the dark matter distribution.

The MICE simulation consists of a light cone of an eight of the sky area $\left(5,156 \mathrm{deg}^{2}\right)$, with redshifts up to 1.4 . It was constructed in a $3 G p c / h$ box with $7 \times 10^{10}$ particles and mass resolution of $2.93 \times 10^{10} M_{\odot} h^{-1}$. In this simulation we will analyze the validity of the halo abundance and the sample variance. Using different approaches to measure and estimate the sample variance of areas of different sizes, we can determine what is the best approach to obtain a reliable covariance of halos.

The DES simulations are larger than MICE, consisting of a quarter of the sky area $(10,313$ $\operatorname{deg}^{2}$ ) and redshifts from $z=0$ up to $z=2$. They consists of a large group of dark matter simulations, in boxes with a few $G p c / h$ wide and mass resolution of $4.938 \times 10^{10} M_{\odot} h^{-1}$, that are latter converted to light cones. In this work, we evaluate the Aardvark v1.0 version of the simulation. The theoretical prediction of the abundance and covariance of halos will also be cross checked in areas of different sizes. However, our main goal for this simulation will be to estimate cosmological parameters. Using the dark matter halos, we can derive idealized constraints on cosmological parameters. Finally we run the WaZp cluster finder on the galaxy catalog associated to these simulation to evaluate the observational effects. Once these effects have been mapped out, we use them to obtain a more robust prediction for the abundance of 
galaxy clusters.

\subsection{MICE SIMULATION}

This simulation was made by the Marenostrum Institut de Ciencias de l'Espai (MICE) (Fosalba et al., 2015b; Crocce et al., 2015; Fosalba et al., 2015a), based on the GADGET code (Springel, 2005). It consists of $\approx 70$ billion particles in a volume of billions of light years aside, resulting in the largest cosmological dark matter simulations to date.

Hoffmann et al. (Hoffmann et al., 2015) re-calibrated the Tinker mass function with the MICE simulation, allowing for predictions that better describe the MICE results. In collaboration with Kai Hoffmann and Fabien Lacasa, we made comparisons between measurements of abundance and covariance of halos and the theoretical predictions. A redshift slice of $0.5 \leq z \leq 0.6$ was chosen to investigate the effects on the mass only. The remaining halos were then binned in mass bins from $10^{12} M_{\odot} h^{-1}$ in $7 \log$ bins of $\Delta \log M=0.3\left[\log M_{\odot} h^{-1}\right]$.

Using healpy, a python implementation of healpix (Gorski et al., 2005), the survey was divided in 96 angular pixels of $\approx 53 \mathrm{deg}^{2}$. Once the pixels with significant border effects were discarded, the remaining 88 pixels were used to measure the abundance and the total covariance on the counts. The results are in Figs. 6.1 and 6.2, with average the number counts in the $\approx 53 \mathrm{deg}^{2}$ cells on the left panel and the counts total variance (diagonal terms of the total covariance matrix) on the right. In both panels, the cyan curves are the measurements from Hoffmann, our measurements are in blue points, and the red line is our theoretical prediction, using the Hoffmann mass function (Eq. 2.32), Tinker bias and the approximation from Eq. 2.53 for the sample variance. In the number counts, the red shaded area is our total variance. In the bottom panel, the difference relative to our measurements is shown. Our measurements are in agreement with those from Hoffmann at a $2 \%$ level, and so is our theoretical prediction at masses $\leq 10^{14.25} M_{\odot} h^{-1}$. For higher masses, the difference is up to $15 \%$, however, in this regime the total variance becomes of the order of the halo counts.

For the covariance panel on the right, the dotted line is the contribution from Poisson noise, the dashed line is the sample variance and the total covariance is represented by the solid lines. At lower masses $\left(M \leq 10^{14} M_{\odot} h^{-1}\right)$, where the number of halos is large, the total covariance is dominated by sample variance. On the other mass limit, Poisson noise dominates. This comes from the fact that sample variance scales as $N^{2}$, while Poisson noise scales as $N$, where $N$ is 
the number of clusters. Therefore, when $N$ is very large, we can expect the sample variance to dominate the contribution to the total halo covariance, and when $N \lesssim 1$, Poisson noise dominates.

For the covariance, the agreement between our measurements and Hoffmann's degrades up to $20 \%$ at lower masses $\left(<10^{14} M_{\odot} h^{-1}\right)$. This difference could be due to border effects, but it should be investigated further. The theoretical prediction has a much larger discrepancy with differences of up to $70 \%$. We should notice, however, that the Tinker bias (Tinker et al., 2010) was used on the theoretical computation of the sample variance. Since Hoffmann et al. (2015) have shown that the Tinker parameters of the mass function are not the best fit for the MICE simulation, it is expected that the Tinker bias will not be a good fit either. Therefore, the parameters of the bias should also be re-calibrated in order to have a fair comparison with the measured covariance. Additionally, we have checked that the approximations used to compute the covariance (Eq. 2.46) present small deviations from the full calculation (Lacasa et al., 2016).

In the process of measuring the covariance from the data itself, we found it useful to consider different measurement techniques, which allowed us to study the robustness and consistency of these measurements. Four methods were analyzed: (SS) Sub-sampling the survey into smaller pieces of equal area and measuring the covariance with these new samples. Note this approach measures the covariance associated to the area of the smaller pieces; ( $\mathrm{SSr}$ ) Re-scaling the measurements of SS by the number of samples; (JK) Using the Jack-Knife method; (BS) Using the Bootstrap method. Both JK and BS are techniques that attempt to automatically estimate the total covariance of the full survey area. To test the validity of each method, we also used three approaches to divide the survey: (N4) Dividing the total area into 24 pieces of $\approx 214 \mathrm{deg}^{2}$; (N16) Dividing the total area into 284 pieces of $\approx 13 \mathrm{deg}^{2} ;(\mathrm{N} 4 \mathrm{~S})$ Dividing the total area into 24 pieces of $\approx 214 \mathrm{deg}^{2}$ and sub-dividing each piece into 16 smaller sub-pieces of $\approx 13 \mathrm{deg}^{2}$. Interestingly, in the latter case we can estimate the covariance of the $214 \mathrm{deg}^{2}$ area in two ways: from SS of the 24 pieces, and from SSr/JK/BS of the $13 \mathrm{deg}^{2}$ sub-pieces comprising 1 piece. This is specially useful to test whether methods that use sub-pieces can indeed provide good estimates of the total area under consideration.

In Fig. 6.3, the different methods are distinguished by the line style, and the different survey divisions by color. The theoretical prediction is displayed by gray lines according to the different areas. As stated before (and in Fig. 6.2), this prediction suffers from not having a fitted bias to MICE simulations, and the total covariance is over-predicted theoretically. Nonetheless, it 

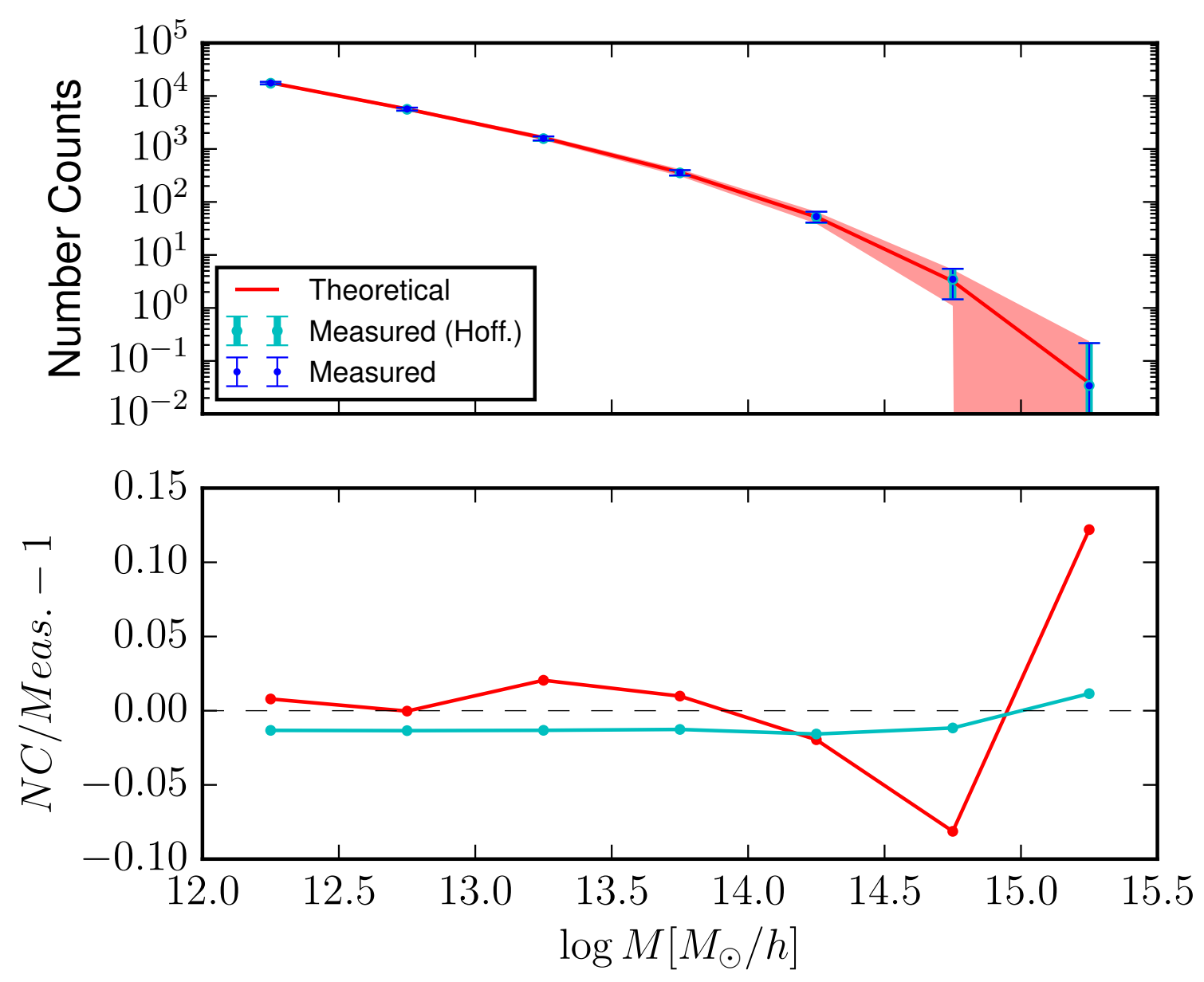

Figure 6.1: Number counts in the MICE simulation for halos with redshifts $0.5<z<0.6$. The red line is the theoretical prediction using Hoffmann mass-function, the blue points are our measurement and the cyan points are the measurements from Hoffmann. The bottom panels are the relative difference with our measurements, with the colors dictated by the upper panels.

can be used as a guideline to determine the scale of the total covariance in different pixel areas.

The results for SSr, JK and BS always converge to the same values, with their respective lines always superposing. The SS methods for both N16 and N4S have the same results, because the area of the resulting pieces used are the same $\left(\approx 13 \mathrm{deg}^{2}\right)$. These results are also close to the theoretical prediction for the area of the smaller pieces. The SSr, JK, BS measurements on $4 \mathrm{~S}$ (blue lines) and 16S (red lines) was in agreement with each other, and also had the same order of the prediction for the full simulation area.

At the scale of the prediction for $214 \mathrm{deg}^{2}$ are the SS method in N4, in which the pieces also have an area of $214 \mathrm{deg}^{2}$, and the SSr, JK, BS methods in N4S, where the estimation was made for each bigger piece of $\approx 214 \mathrm{deg}^{2}$ but using the smaller pieces of $\approx 13 \mathrm{deg}^{2}$. However, using $\mathrm{SSr}, \mathrm{JK}$, BS seems to have a consistently lower covariance than the SS, being $\approx 15 \%$ lower at 

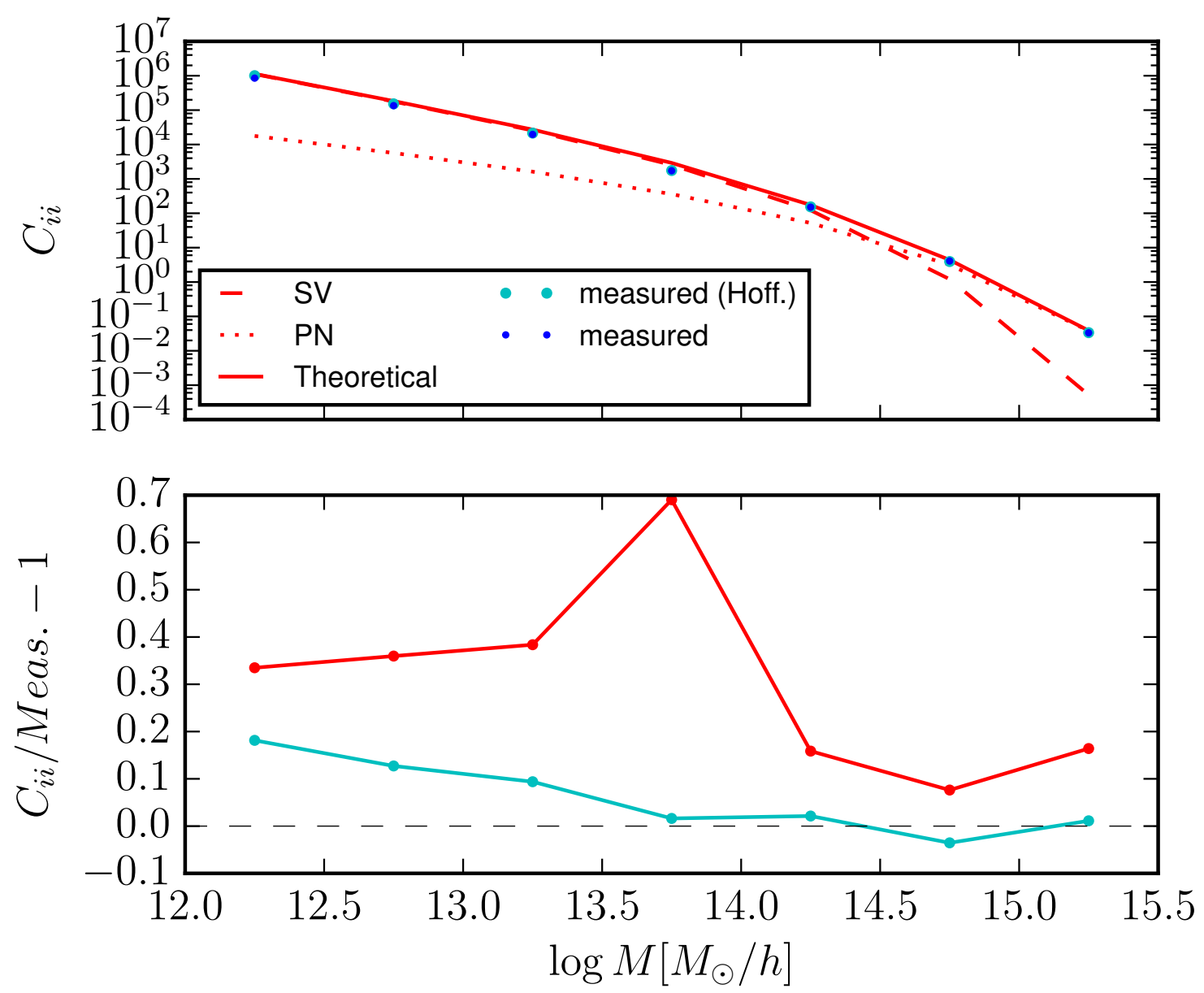

Figure 6.2: Total variance in the MICE simulation for halos with redshifts $0.5<z<0.6$. The red lines are the different components of our theoretical computation of the covariance using Hoffmann mass-function and Tinker bias, with the red dotted line being the Poisson noise (PN), the red dashed line is the sample variance (SV) and the red solid line is the total covariance. The blue points are our measurements and the cyan points are the measurements from Hoffmann. The bottom panels are the relative difference with our measurements, with the colors dictated by the upper panels.

lower masses $\left(<10^{13.5} M_{\odot} h^{-1}\right)$ and $\approx 10 \%$ lower at higher masses. From these results, we can infer that SSr, JK, BS methods could be used to get a rough estimate of the covariance in the $5,156 \mathrm{deg}^{2}$ area, even though they do not consider fully the cosmic variance effects.

\subsection{DES Aardvark v1.0 SIMULATION}

In the DES context, the simulation studied was the Aardvark v1.0 catalogs of the Blind Cosmology Challenge (BCC). It is a dark-matter particle simulation with catalogs for halos and galaxies, containing both their true and observed properties. The BCC simulations were developed 


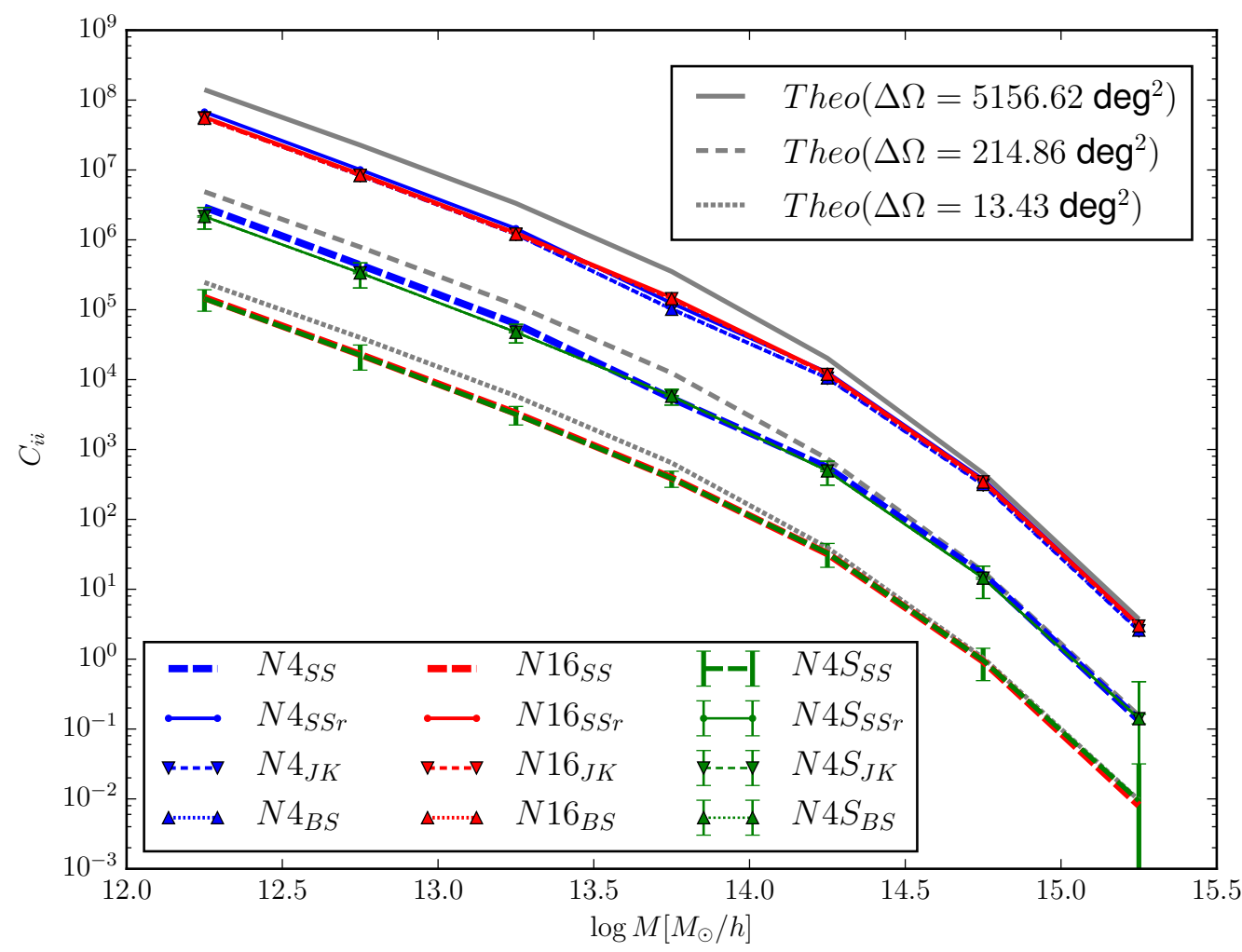

Figure 6.3: Total variance measured in the MICE simulation with different methods and subdivisions, along with theoretical predictions. The theoretical predictions is displayed in gray lines with the style changing with the area. The measurement methods are separated by line styles as: Sub-sampling (SS); Sub-sampling re-scaled (SSr); Jack-Knife (JK); Bootstrap (BS). The colors distinguish the area splitting method: (N4) blue ; (N16) red ; (N4S) green .

from and for the DES collaboration in order to test multiple pipelines, and in particular codes for constraining cosmological parameters. The cosmology used to construct the simulation was known but not revealed during the challenge (for which reason it was a blind challenge).

The statistical and theoretical analysis directly using the dark matter halos will provide a testbed and baseline for checking the cosmological constraints framework. This step is extremely important as it will provide the best constraints possible and determine what issues may appear on the predictions. Each unsolved issue related to the prediction for dark matter halos will be carried on to the prediction for galaxy clusters, where many more effects have to be studied. The WaZp cluster finder results run on the galaxy catalog will be evaluated in detail, allowing us to verify different effects that must go into the predictions of cluster abundance. 


\subsubsection{Measurement of Halo Abundance and Sample Variance}

The halos provided by the DES simulation were detected with the Rockstar halo finder (Behrooz) et al., 2013, 2012), an algorithm based both on SO and FoF techniques. The catalog produced was complete above $\approx 4.5 \times 10^{13} M_{\odot} h^{-1}$, i.e. it detected all halos above this threshold, and included halos down to $\approx 5 \times 10^{12} M_{\odot} h^{-1}$ at low redshifts totaling $\approx 30$ million objects.

The covariance was measured for halos with $M>10^{13.8} M_{\odot} h^{-1}$ in 5 mass bins of $\Delta \log M=$ $0.3\left[M_{\odot} h^{-1}\right]$ and 20 redshift bins of $\Delta z=0.1$ from $z=0$ to $z_{\max }=2.0$. Using healpix, the survey was sub-divided into 12, 48, 768 and 49152 pixels of $858 \mathrm{deg}^{2}, 214 \mathrm{deg}^{2}, 13 \mathrm{deg}^{2}$ and $0.21 \mathrm{deg}^{2}$, respectively, in order to analyze the effect of having more independent samples for the measurements. In Fig. 6.4, we show the halo number counts measured in blue points and the theoretical predictions using the Tinker mass function as the red lines, where the error bars are the measured covariance based on the pixels. Each line corresponds to a different mass bin indicated in log scale on the top right. The red shaded area is the total theoretical halo covariance computed with the Tinker bias and the cylindrical window approach of Eq. 2.53. In all areas considered, the theoretical predictions of the abundance had a small deficiency at the peak of the distribution $(z \approx 0.75)$ for $13.8 \leq \log \left(M / M_{\odot} h^{-1}\right) \leq 14.1$, when compared to the data. For the larger areas, the total variance is relatively smaller, and this discrepancy can lead to a significant bias on cosmological parameters. 

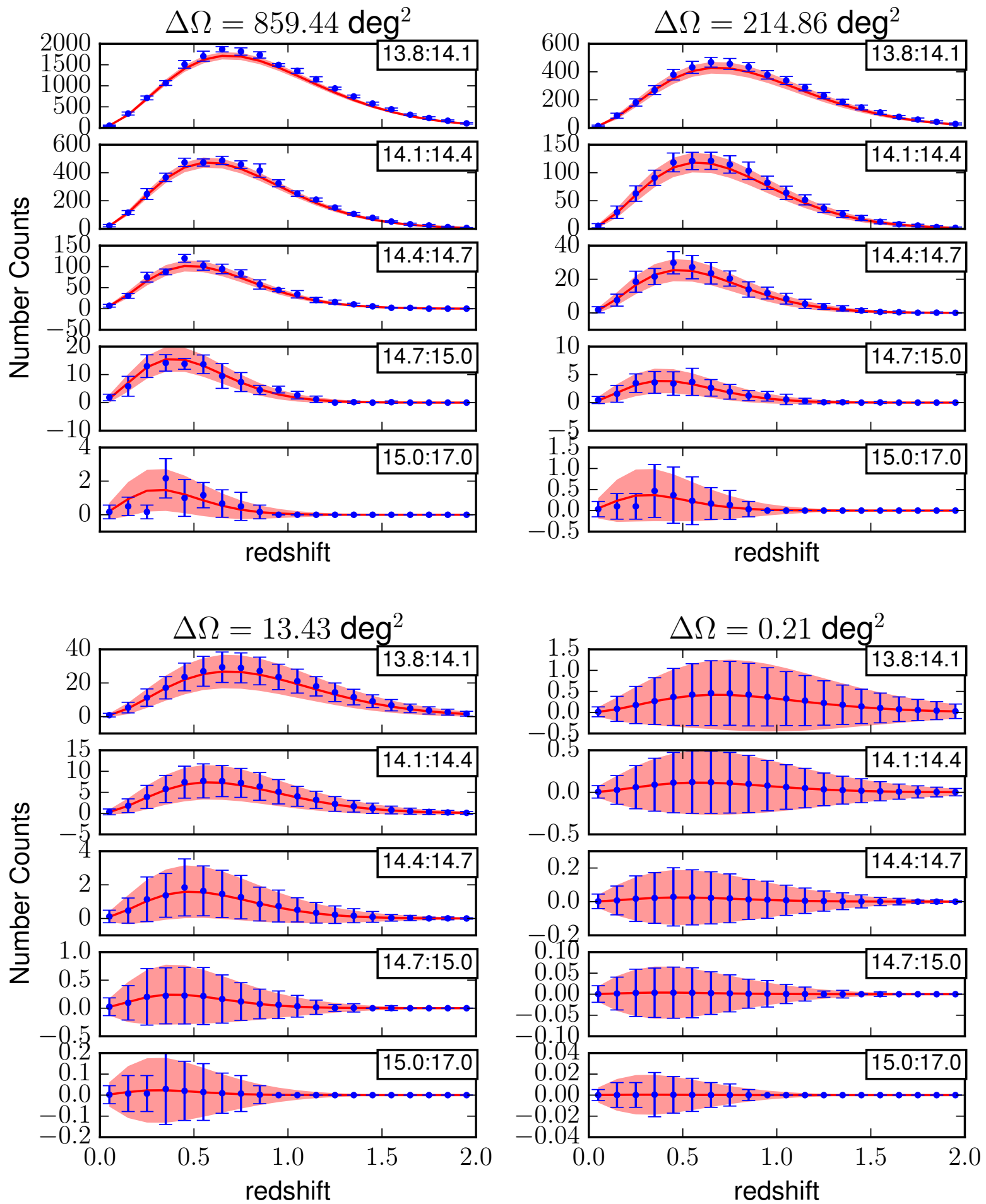

Figure 6.4: Number counts measured in the Aardvark v1.0 simulation along with the theoretical prediction with different areas and mass bins. The blue points and error-bars are the measurements for the average counts and their total variance, respectively, whereas the red lines and shaded regions are the theoretical predictions for the counts and total covariance, respectively. The mass bins are displayed on the top right corner of each sub-panel in units of $\log \left(M / M_{\odot} h^{-1}\right)$.

It is important to observe that in this section we are using each sample of pixel with different 
areas to estimate the total covariance of the pixels, not the covariance of the whole simulation (of $\Delta \Omega \approx 10,313 \mathrm{deg}^{2}$ ). Hence, we will not be affected by effects of the covariance estimator, detailed in section 6.1. The correlation matrix for each area is shown in Fig. 6.5, where each bin index can be computed by $i_{\text {bin }}=20 i_{\text {mass }}+i_{z}$. The black regions are bins with null counts, that result in null covariance and indefinite correlation. As expected, the highest correlations appear on the diagonal, which corresponds to the auto-correlations. Then we have correlations of same mass bins, with different redshift bins having a very small correlation. As we make more sub-divisions, the areas become smaller, but the number of samples to estimate the covariance increases, and the signal-to-noise ratio is higher. It can be seen that for the area of $858 \mathrm{deg}^{2}$, where there are only 48 angular samples, the correlation matrix is very noisy. By increasing the number of samples the signal becomes more visible, up to our limit case of 49,152 samples, where the noise is quite small.
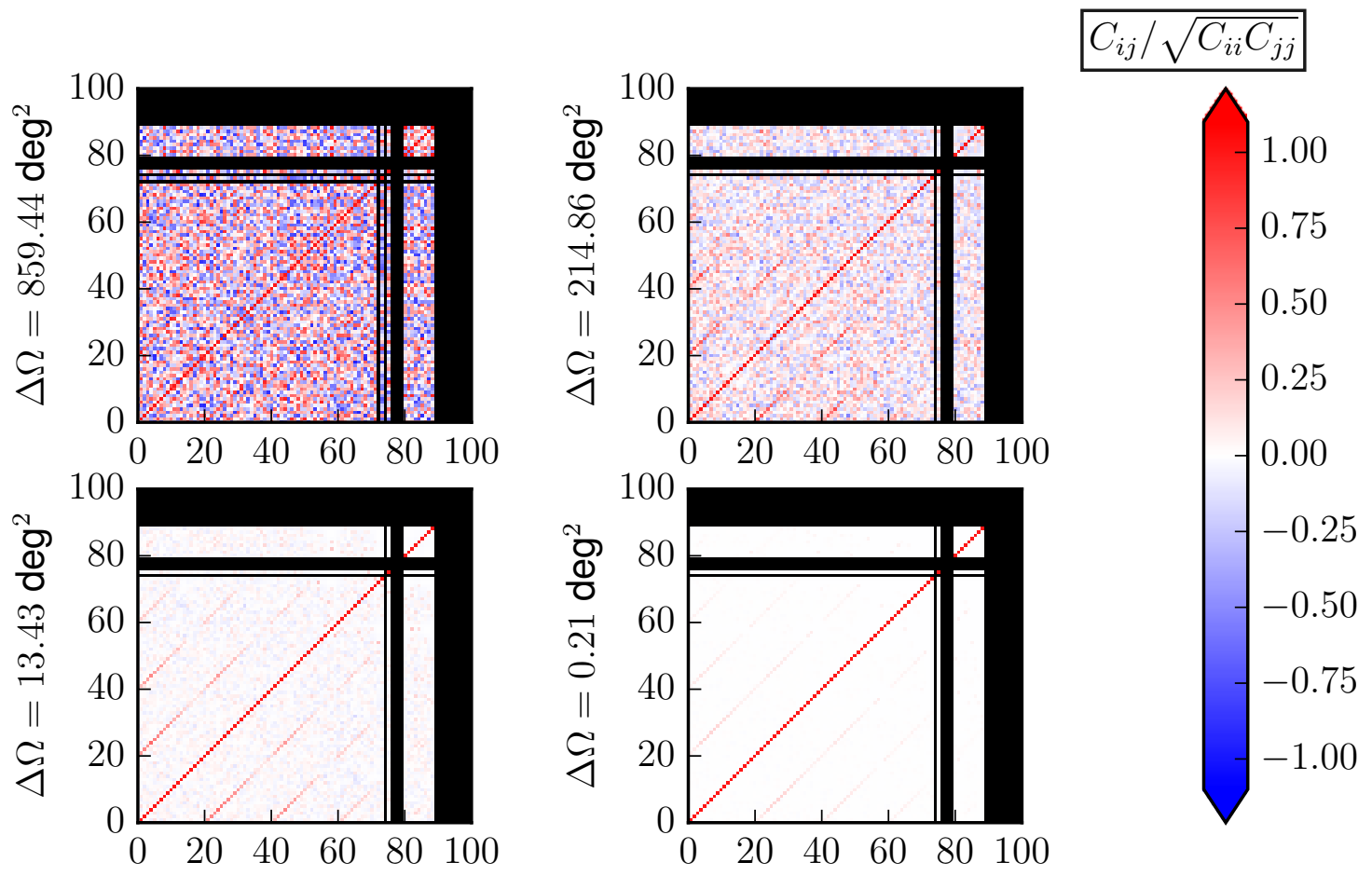

Figure 6.5: Total variance measured in the DES Aardvark v1.0 simulation using the multiple samples created.

The relative difference between the measured and the predicted covariance matrix can be seen in Fig. 6.6. In every case, the more prominent measured correlations described by the auto-correlations and from bins with the same mass are in better agreement with theoretical predictions. For cases with more sub-samples the measurements become closer to the predic- 
tions. However, even at the case with most samples, there is a $40 \%$ discrepancy at bins with a small redshift difference. The covariance in bins with a large redshift difference can differ from predictions by a factor greater than 2 , though we must consider that these bins involve high redshifts, where the halo abundance is low and shot noise dominated.
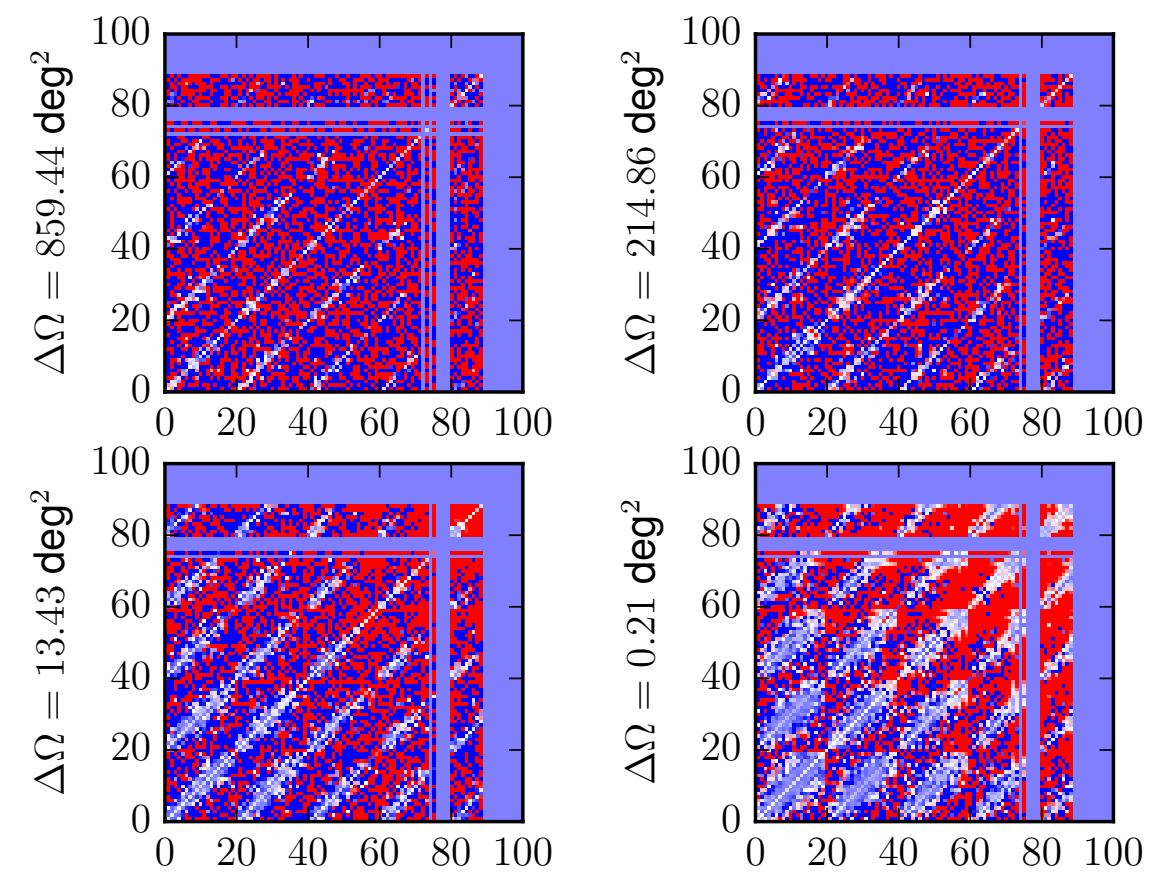

$$
C_{\text {meas }} / C_{\text {pred }}-1
$$

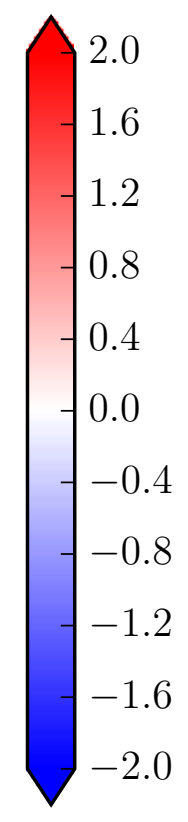

Figure 6.6: Relative difference between total covariance measured in the DES Aardvark v1.0 simulation and theory predictions including Poisson variance and sample covariance.

\subsubsection{Cosmology from Dark Matter Halos}

The maximum possible potential of galaxy cluster cosmology is reflected in the capability of constraining cosmological parameters with perfectly detected dark matter halos. Deficiencies on cosmological constraints from dark matter halos will imply the baseline limitations for cluster cosmology. Additionally, as there are many other observational effects to be considered for the predictions of clusters, it is important that the constraints from halos are well characterized and understood.

Our cosmological constraints were made using halos with $M>10^{13.8} M_{\odot} h^{-1}$ in 5 mass bins of $\Delta \log M=0.3$ and 20 redshift bins from 0 to 2 with $\Delta z=0.1$, assuming the Tinker mass function for the prediction. We split the whole survey area into 768 pixels of $214 \mathrm{deg}^{2}$ in order to have robust covariance measurements, as seen in the last section. The constrained 
cosmological parameters were obtained using a MCMC method and also with Fisher Matrix forecasts. We allowed variations in cosmology to change only the predicted number counts, but kept the covariance fixed at its measured values. Again, we remind that the covariance measured here is computed using several pixels with the considered area $\left(214 \mathrm{deg}^{2}\right)$ and are not affected by a covariance estimator. We considered two cases which analyzed different variation of cosmological parameters.

In Fig. 6.7 we show cosmological constraints for cosmology where parameters $\left(h, n_{s}, \Omega_{b}\right)$ were kept fixed, while $\left(\Omega_{m}, w, \sigma_{8}\right)$ were allowed to vary freely. The two values on the inset are the best fit and the mean value of the chain. The filled regions are the forecasts using Fisher Matrix approach, and the solid lines are the MCMC results from the catalog measurements. The constraints on $\left(\Omega_{m}, w, \sigma_{8}\right)$ were of $(7 \%, 9 \%, 1 \%)$ and the fitting values agree with the simulation cosmology at a $1 \sigma$ level. In fact, the constraints from the MCMC estimation were remarkably close to the Fisher Matrix forecasts, including the correlation between the cosmological parameters.

In Fig. 6.8, all 6 cosmological parameters $\left(\Omega_{m}, w, \sigma_{8}, h, n_{s}, \Omega_{b}\right)$ parameters were allowed to vary freely. The resulting constraints were of $(10 \%, 10 \%, 2 \%, 10 \%, 7 \%, 46 \%)$, a small degradation for $\left(h, n_{s}, \Omega_{b}\right)$ compared to the previous case. With the exception of $\Omega_{b}$, these results seem to imply that all parameters could be well constrained. However, for $\left(h, \Omega_{b}\right)$, the best fit and mean value of the MCMC chain do not agree very well within $1 \sigma$ of the true values. This indicates that an external prior should be used for these parameters, such as from different probes of cosmology or different surveys.

In this case, the Fisher Matrix forecasts differ somewhat from MCMC predictions. This could be attributed to the fact that a flat prior imposing $\Omega_{b}>0$ was used in the MCMC method, as a negative value of $\Omega_{b}$ does not have physical meaning. In the bottom right panel of Fig. 6.8, we can see the sharp cut at zero for the $\Omega_{b}$ distribution, resulting in an artificial improvement on the $\Omega_{b}$ constraint. Consequently, the parameters correlated with $\Omega_{b}$ also have better constraints than forecasted with Fisher Matrix. A similar effect, albeit of smaller magnitude, occurs with $h$ and the flat prior $h>0.2$. When we applied a strong prior on both parameters $\sigma_{p}\left(\Theta_{\left\{h, \Omega_{b}\right\}}\right)=5 \%$, the resulting MCMC constraints are lager than the Fisher Matrix forecasts.

In Fig. 6.9, we show the halo number counts measured along with the best fit found in the two cases above of parameter constraints. The predicted abundance of halos matches closely each measurement, specially when all parameters were allowed to vary. There is a small dif- 




Figure 6.7: Cosmological constraints with dark matter halos in the BCC simulation. The parameters $\left(h, n_{s}, \Omega_{b}\right)$ were fixed at their fiducial values. The filled regions are the forecasts using the Fisher Matrix and the solid lines are the results from the MCMC on the catalog measurements.

ference, smaller than the $1 \sigma$ scatter of the data, between the number of halos measured and predicted with 3 parameters for $\log M_{t h}=[13.8: 14.2]$. However, such a small difference can be expected from random fluctuations, as we have only one simulation representing a single realization of the Universe for the given cosmology.

\subsubsection{WaZp Clusters}

Along with the dark matter halo catalog, the BCC Aardvark v1.0 simulation produced a galaxy catalog, where it was possible to run cluster finders to detect galaxy clusters and make a direct comparison between clusters and halos. Although the catalogs have a wide coverage of $\approx 10,313 \mathrm{deg}^{2}$, the WaZp code was run on a reduced area $\left(\approx 220 \mathrm{deg}^{2}\right)$ in order to check its performance. The redshift range was limited to $0.1 \leq z \leq 1.0$, to have similar conditions of the observed data from the DES first year release. In this region, there were 45,677 dark matter halos with mass above $10^{13} M_{\odot} h^{-1}$. 


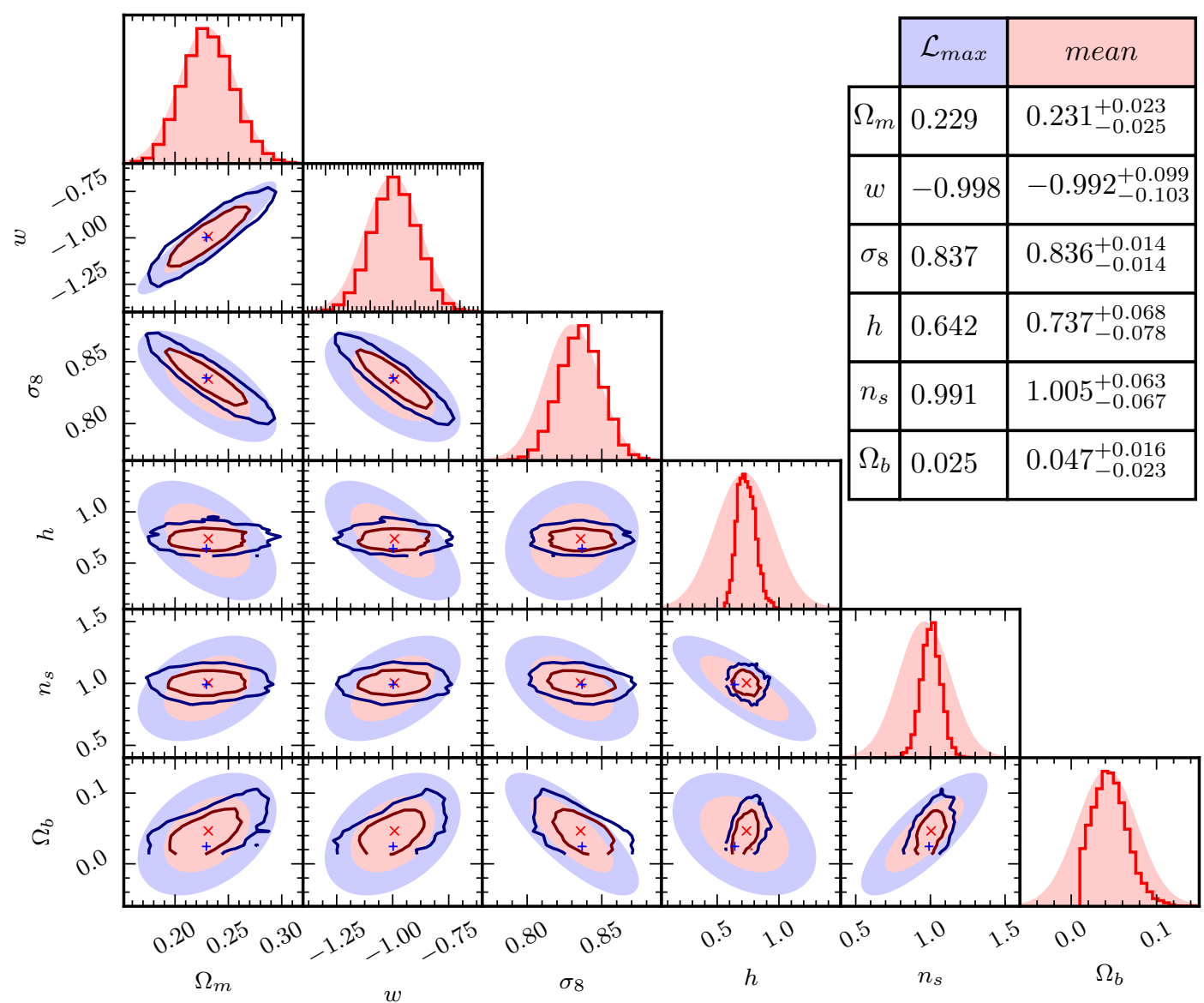

Figure 6.8: Cosmological constraints with dark matter halos in the BCC simulation. The filled regions are the forecasts using the Fisher Matrix and the solid lines are the results from the catalog measurements.

The WaZp cluster finder was run on the galaxies with Gaussian photometric redshifts created in the Aardvark v1.0 simulation, and the thickness of each slice was determined according to uncertainties of the photo-zs. These redshifts were created based on Gaussian errors around the real redshifts. Although these errors do not reproduce the complex features of photometric redshifts on real data, they can be used as a starting point to evaluate observational redshift effects. Our goal is to step up to realist photo-zs in latter works, also available in the simulation. The mass-proxy for WaZp clusters is the richness, computed by integrating over the probability that each nearby galaxy belongs to each cluster. Therefore, even though the richness is related to the number of galaxies "belonging" to each cluster, its value is not necessarily an integer. In the footprint considered, 39,861 galaxy clusters were detected with richness above 3 . The angular density distribution of WaZp clusters and the dark matter halos in the reduced region are shown in Fig. 6.10,

Before matching halos and clusters, it is useful to investigate their distributions across 


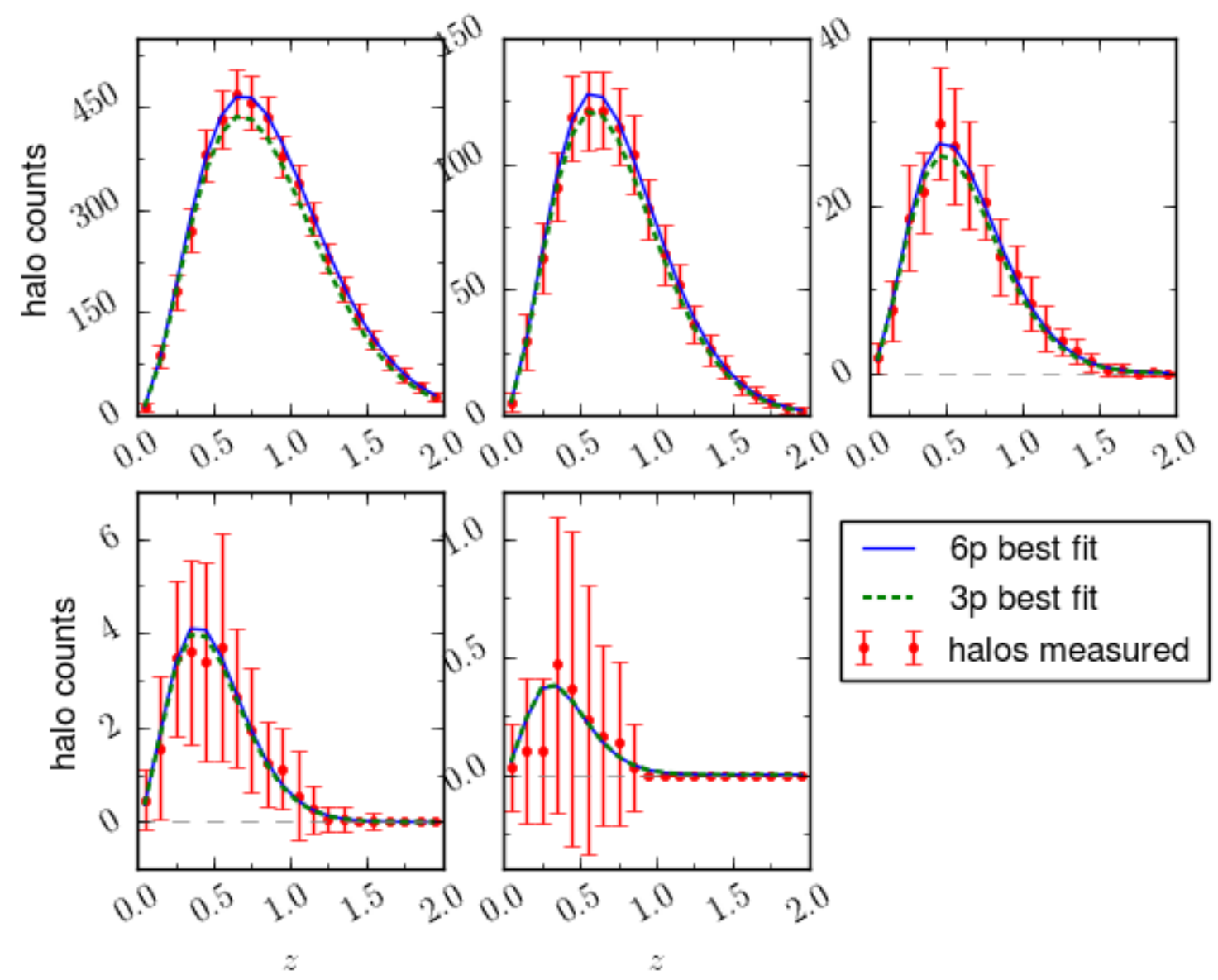

Figure 6.9: Halo number counts (red points) measured in the BCC simulation and theoretical predictions at the the best fit parameters found in the MCMC chain (lines). The blue solid line is the result of allowing all cosmological parameters to vary in the chain, and the green dashed line is for the case where only $\left(\Omega_{m}, w, \sigma_{8}\right)$ vary.

mass/richness and redshift as shown in Fig. 6.11. For both halos and clusters, the overall distribution is similar, with the number of objects decreasing at higher mass/richness and a deficiency at lower redshifts $(z \lesssim 0.3)$. However, the distribution of clusters does not present the same decrease of the halos at high redshift $z>0.9$, which could lead to a decrease in purity at this reshift range.

\section{Matching Clusters and Halos}

Halos and clusters were matched by proximity with mass/richness priority. As the angular positioning of clusters - computed from the angular position of member galaxies - and the redshift determination are separate processes with different precisions, it is useful to split the matching criteria into redshift and angular distance independently, instead of computing a 3D radial distance. The resulting condition becomes a cylinder around the halo/cluster to be matched, with 

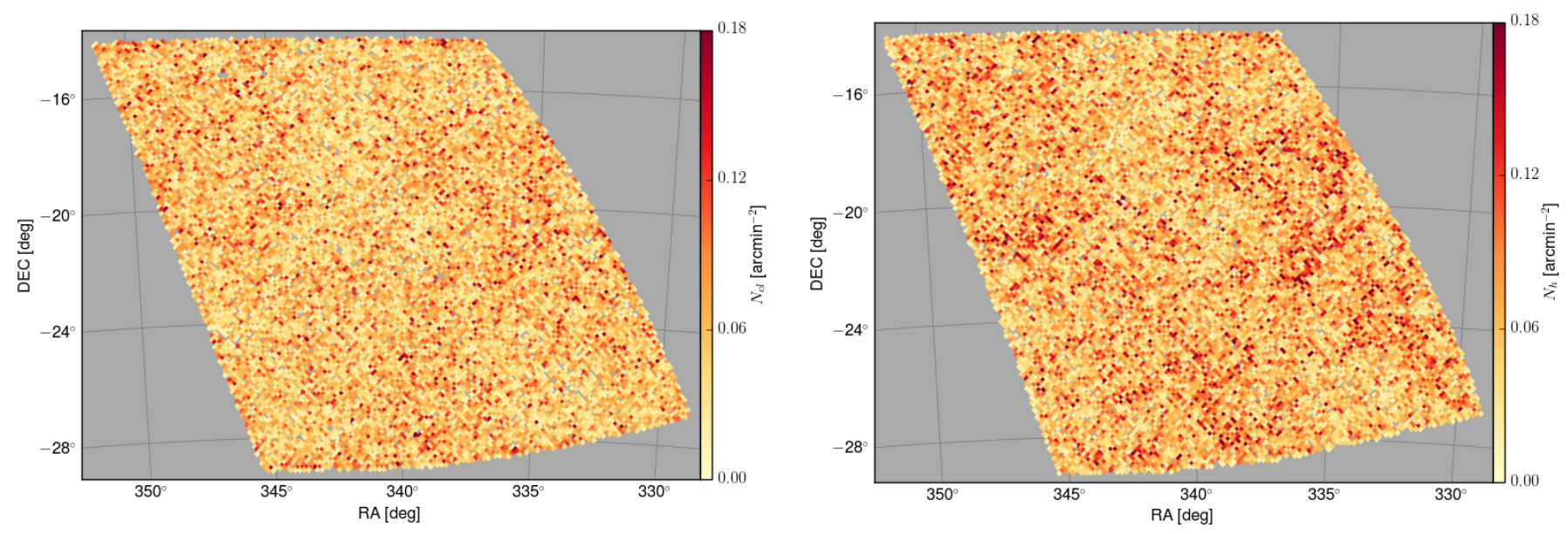

Figure 6.10: Angular density of matter halos (left) and WaZp clusters (right) of the Aardvark v1.0 catalog of the BCC simulation.

dimensions related to the uncertainties on redshift and angular positioning of the clusters. The criteria adopted for the matching was

$$
\begin{cases}\left|z_{\text {halo }}-z_{\text {cluster }}\right| & \leq \sigma_{z}(1+z) \\ \Delta \theta & \leq N \theta_{R}=2 N \arcsin \left(\frac{R}{2 D\left(z_{m}\right)}\right)\end{cases}
$$

where $\Delta \theta$ is the angular distance between the cluster and halo centers, $D\left(z_{m}\right)$ is the comoving distance to the average redshift $z_{m}$ of the cluster-halo pair $z_{m} \equiv\left(z_{\text {halo }}+z_{\text {cluster }}\right) / 2$, and $\sigma_{z}\left(\sim 10^{-1}\right)$ and $N(\sim 1)$ are matching parameters. The functional form $\sigma_{z}(1+z)$ reflects the expectation of photo-z errors increasing with redshift (Ilbert et al., 2006; Mazure et al., 2007; Arnouts et al., 2007; Ilbert et al., 2009), while $\theta_{R}$ is the angular size of the halo/cluster at the average redshift $z_{m}$. If the physical radius of the halos/clusters $(R)$ is provided instead of the angular radius $\left(\theta_{R}\right)$, the matching will require computation of comoving distances, and a choice of cosmology must be made. In the present case of simulations, the values of the cosmological parameters are known. However, for the real data they would have to be assumed. In the latter case, after the cosmological analysis we would have to check if the parameters found are in agreement with those assumed.

The matching parameters must be carefully selected, as a poor choice that is too loose can lead to wrong halo-cluster pairs to be matched (lowering purity) and a choice that is too restrictive will exclude some correct matches (lowering completeness). To evaluate the best choice of parameters, we repeated the matching for a wide range of values for $N$ and $\sigma_{z}$, as 

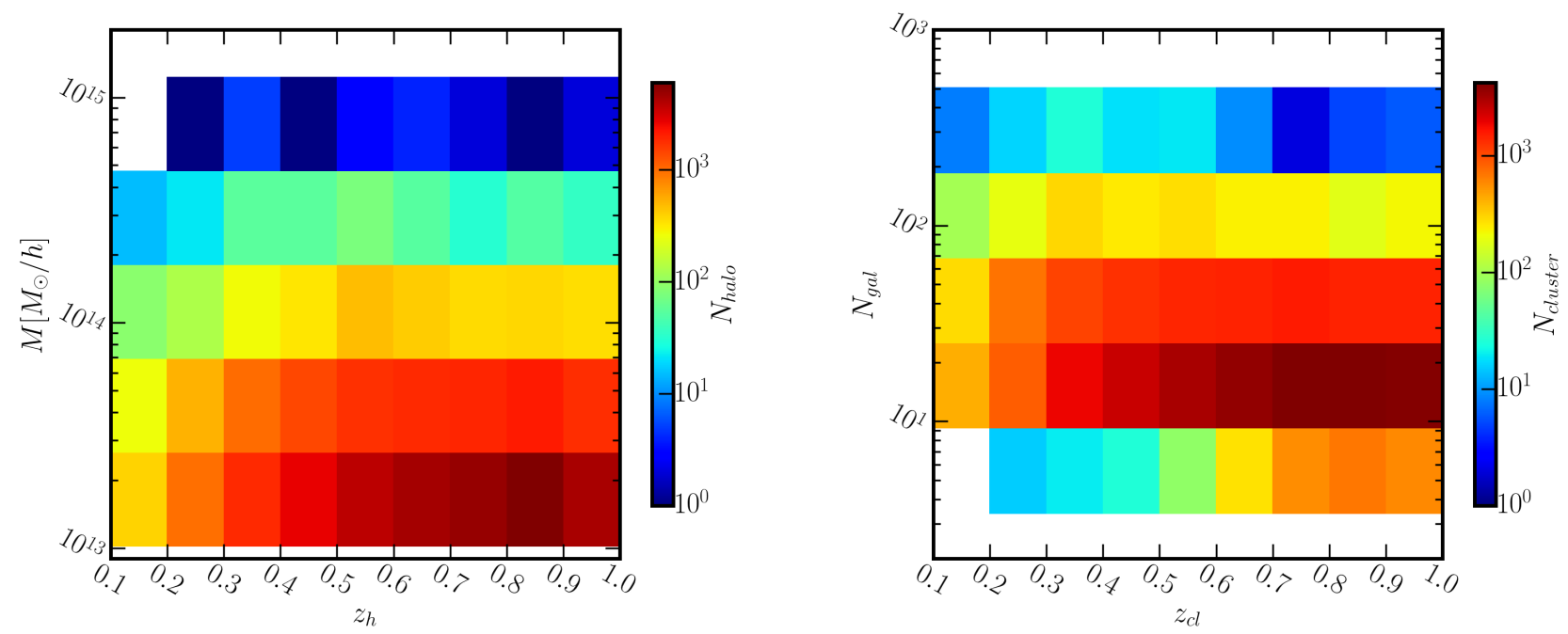

Figure 6.11: Distribution of dark matter halos (left) and WaZp clusters (right) in the Aardvark v1.0 catalog of the BCC simulation as a function of mass/richness and redshift.

shown in Fig. 6.12, In the left panel, the number of halos (top panel) and clusters (bottom panel) matched allowing multiple matches (case 3 of Table A.1) is displayed. This matching case does not provide a one-to-one correspondence between halos and clusters, as the same halo can be matched to several clusters and vice-versa. Ultimately, this matching reports that a halo (cluster) has a cluster (halo) in the vicinity, defined by the matching parameters. As a result, the number of objects matched only increases with increment on the matching parameters from $\approx 17 k$ halos $(\approx 15 k$ clusters $)$ matched with $\left(\sigma_{z}, N\right)=(0.025,0.5)$ to $\approx 40 k$ halos $(\approx 35 k$ clusters) matched with $(0.25,3.5)$. Naively, we could think that the same behavior would follow for all cases of matching. However, this does not happen for cross matching of the exclusive matching (case 6 of Table A.1), as can be seen in the right panel of Fig. 6.12 .

When the matching parameters are too restrictive, i.e. lower than errors/uncertainties on the redshift and angular centering of the clusters, several "true" halo-cluster pairs are not matched and the number of matched objects is lower. On the other extreme, when the parameters are too loose, the number of matched objects found on the halo exclusive matching and cluster exclusive matching increases, yet most of the pairs found in each matching differ and, upon requiring cross match, the number or paired objects also decreases. Hence, the parameters that maximize the number of cross matched objects $\left(\sigma_{z}, N\right)=(0.075,1.0)$ seem to be well defined and will be used as the default choice. The several types of matching results, as discussed in section A.1, are displayed in Table 6.1. 



Figure 6.12: Number of dark matter halos and WaZp clusters matched in the BCC simulation with different matching parameters. In the left are the number of halos (top) and clusters (bottom) matched considering multiple matches and in the right is the number of matched halos and clusters with cross match only.

\begin{tabular}{c|cc}
\hline- & Halos & Cluster \\
\hline Total Number & 45,677 & 39,861 \\
\hline Halo Multiple Match & 32,609 & 30,596 \\
Cluster Multiple Match & 35,454 & 29,820 \\
Multiple Match & $\mathbf{3 6 , 1 7 7}$ & $\mathbf{3 1 , 4 8 0}$ \\
\hline Halo Exclusive Match & 25,921 & 25,921 \\
Cluster Exclusive Match & 26,060 & 26,060 \\
Cross Match & $\mathbf{2 2 , 4 1 6}$ & $\mathbf{2 2 , 4 1 6}$
\end{tabular}

Table 6.1: Results for the matching between WaZp clusters and dark matter halos on the BCC Aardvark v1.0 simulation.

Two indicators of the quality of the matched objects are the distribution of the difference in redshift and in angular centering. In Fig. 6.13, the redshift difference distribution of the matching is displayed in bins of halo mass, cluster richness, halo redshift and cluster redshift. In all cases, the distribution goes to zero quickly as $\Delta z$ goes to $\sigma_{z}(1+z)$, reassuring a good choice of $\sigma_{z}$. Additionally, the distributions are centered at $\Delta z=0$, indicating there is no bias between the halo and cluster redshifts. There is a slight improvement on the more massive/richer bins, as the peak of the distribution is sharper. This could be attributed to the richer clusters having more precise estimates of redshift and richness, as they have more galaxies and are associated to more massive halos. The distribution does not change significantly in the different redshift bins of both halos and clusters, reinforcing the assumption that the scatter on the cluster redshift 



Figure 6.13: Distribution of the matched pairs as a function of normalized redshift distance, binned by mass (top left), richness (top right), halo redshift (bottom left) and cluster redshift (bottom right).

can be parametrized by $\sigma_{z}\left(1_{+} z\right)$, as the $x$ axis of the figures are normalized by $\left(1+z_{m}\right)$.

The distribution of matched pairs for angular distance (converted to physical distance and normalized by the halo radius), is shown in Fig. 6.14, binned in mass, richness, halo redshift and cluster redshift. None of the distributions peaks at zero, indicating a miscentering between cluster and halos. This is caused by the WaZp cluster finder and Rockstar halo finder having different methods to define the centers of clusters and halos. Once again, the bins with richer clusters have a better centering location due to having more member galaxies, and also the more massive bins by association. As we go to higher redshift bins for both halos and clusters, the centering worsens. This behavior can be explained by having the angular precision for all galaxies of the same order, across all redshifts. A fixed angular precision will translate into a 
physical precision that worsens with the distance, and consequently with redshift.
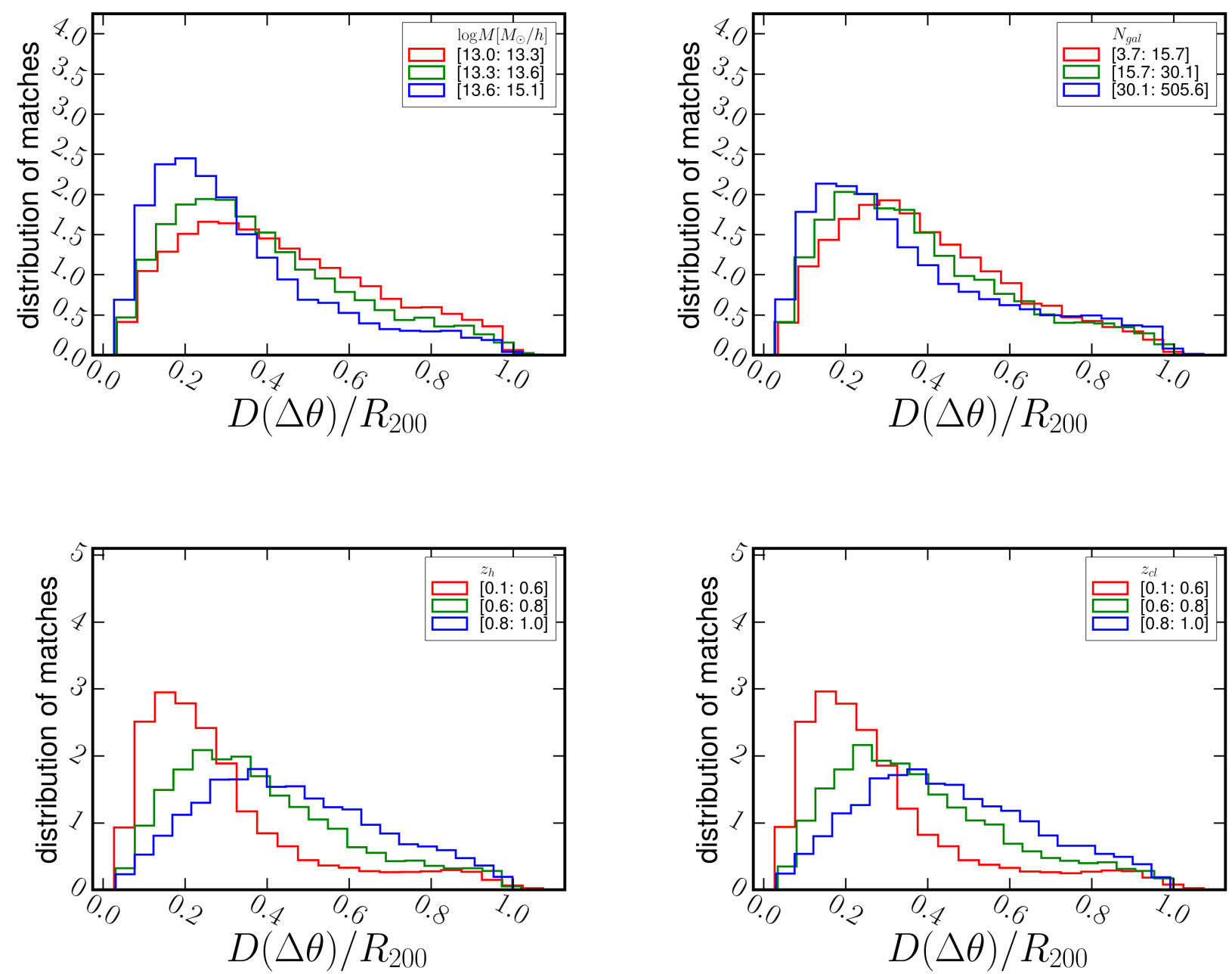

Figure 6.14: Distribution of the matched pairs as a function of angular distance converted to physical distance and normalized by the halo radius $R_{200}$, binned by mass (top left), richness (top right), halo redshift (bottom left) and cluster redshift (bottom right).

\section{Completeness and Purity}

The completeness and purity in the footprint considered are shown in Fig. 6.15. These computations were done using all halos and clusters, without cuts on mass/richness or redshift, and should be viewed to check possible irregularities on the angular footprint. Except for the borders, there is no particular region with a concentration of low completeness and purity, indicating that there was no specific region with worse observational conditions.

The completeness and purity as a function of mass/richness and redshift (see section A.2) are shown in Fig. 6.16 as a color map. The results for completeness and purity were similar, 

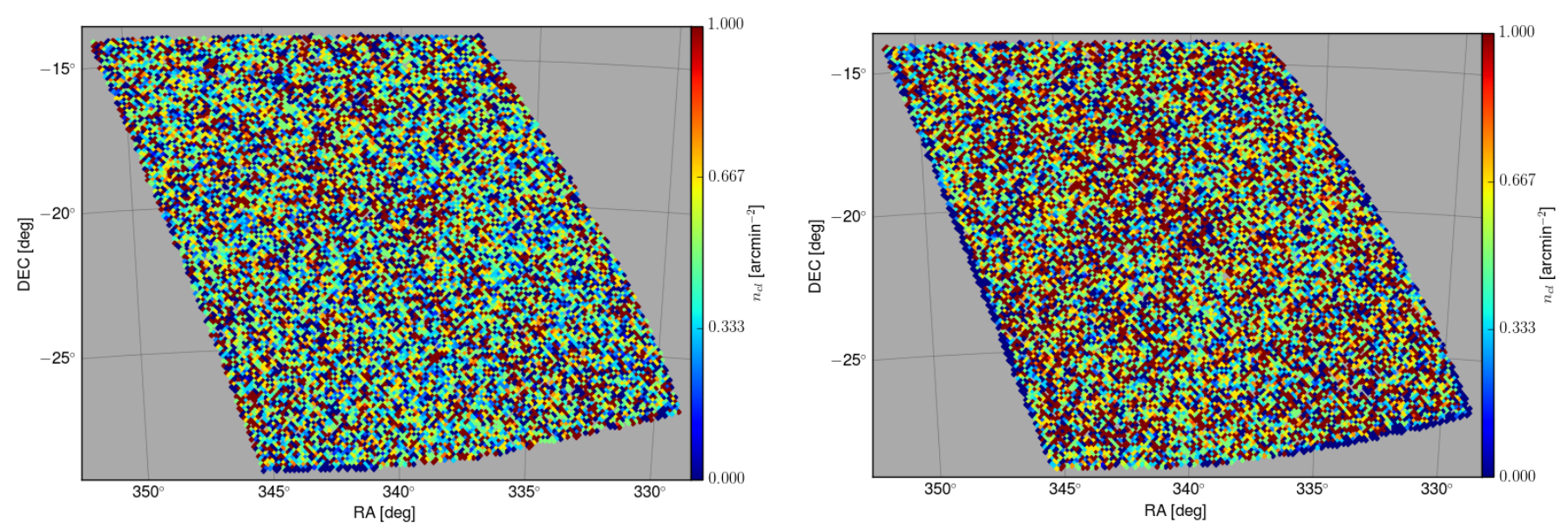

Figure 6.15: Completeness (left) and purity (right) of the WaZp catalog in the BCC simulation by pixels in the footprint.

specially considering a conservative richness cut of $N_{\text {gal }}>10$. The cluster finder appears to function equally well across different redshifts, as the completeness and purity only have minor variations with redshift. Both completeness and purity improve at higher masses/richness, as expected, since clusters with more galaxies should be easier to detect and with more precision. There appears to be a specific deterioration on the completeness at $(M, z) \approx\left(5 \times 10^{14}, 0.1\right)$ and on the purity at $\left(N_{g a l}, z\right) \approx(350,0.65)$, however, in both cases it was verified that there were only two halos/clusters in those regions, thus this decrease is not statistically significant. Over $80 \%$ of halos with masses above $10{ }^{14} M_{\odot} h^{-1}$ were detected as WaZp clusters and over $60 \%$ of the clusters with richness above 30 corresponded to a simulated dark matter halo.

We used the functional forms defined in section 3.3 .3 to fit the completeness and purity, replacing the observed mass $M^{\text {obs }}$ by the richness $N_{g a l}$ and redefining parameters:

$$
\begin{gathered}
c_{0} \rightarrow \log M_{c f i d}+c_{0} \\
p_{0} \rightarrow \log N_{g a l}^{p f i d}+p_{0} .
\end{gathered}
$$

In Fig. 6.17, we show measurements of completeness in redshift and mass bins and purity in $z_{c l}$ and richness bins, as well as the fitted functions. For the error bars, we used a Poissonian approach of $1 / \sqrt{(} N)$, where $N$ is the number of objects in each bin. As we can see from the figure, this functional form seems to describe well the measured completeness and purity of the cluster catalog. For both selection functions, the power indices recovered were close to unit 

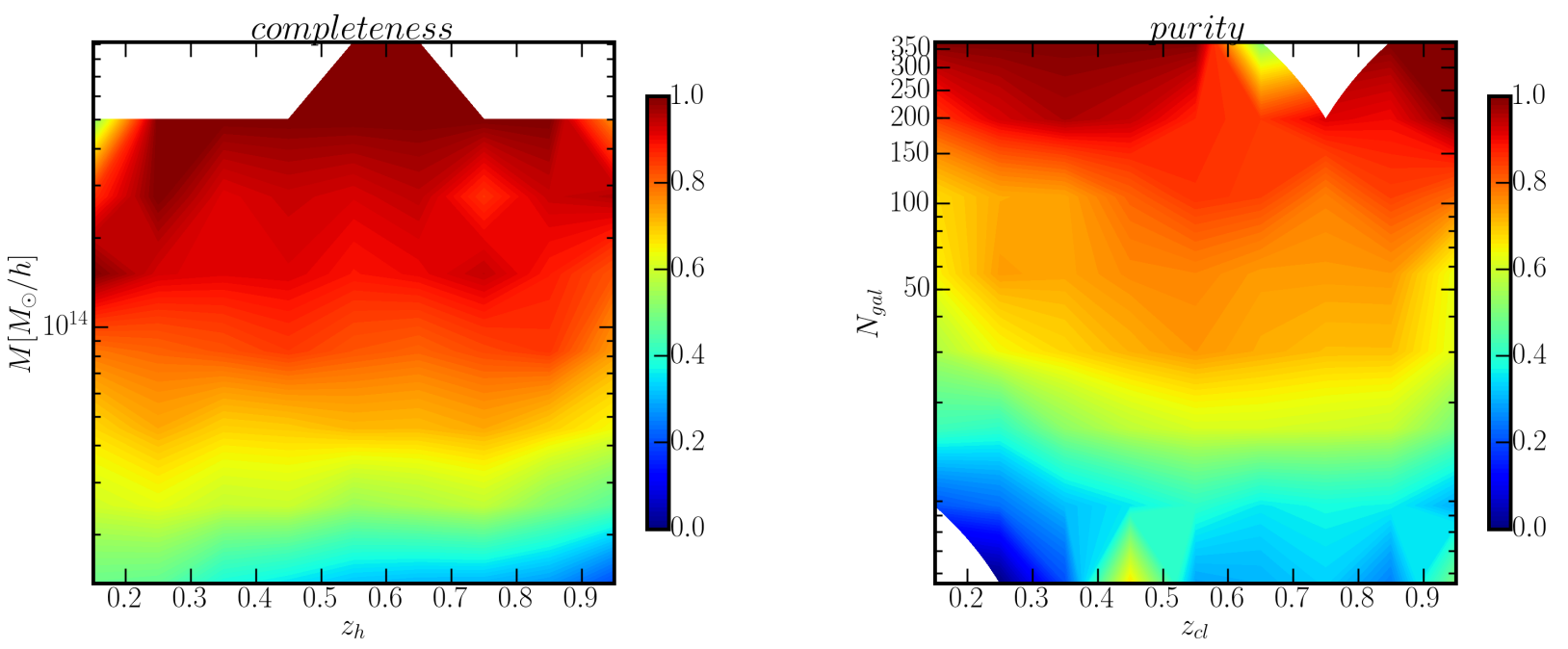

Figure 6.16: Completeness (left) and purity (right) of the WaZp catalog in the BCC simulation by mass/richness and redshift.

$n_{c}=1.023 \pm 0.071, n_{p}=0.955 \pm 0.055$. The completeness scale parameter was found to be $c_{0}=12.71 \pm 0.19$, with a redshift dependency $c_{1}=0.39 \pm 0.11$, resulting in a scaling mass of $\approx 10^{13} M_{\odot} h^{-1}$ which increases with redshift. For the purity, the best-fit scale parameter was $p_{0}=1.48 \pm 0.12\left(N_{\text {gal }} \approx 30\right)$, with a negative redshift parameter $p_{1}=-0.150 \pm 0.078$ leading to a low scaling richness $\sim 20-30$.
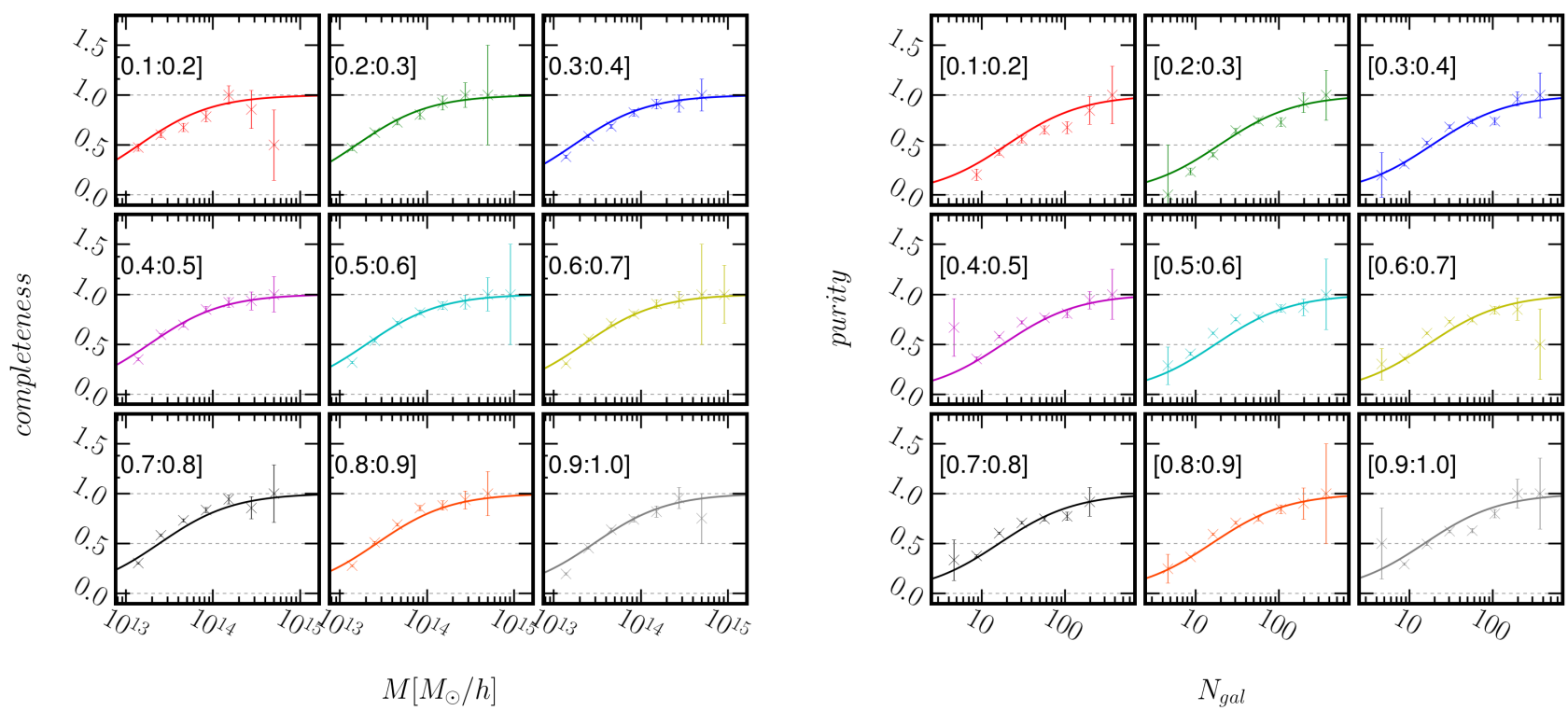

Figure 6.17: Completeness and purity as a function of mass/richness in several redshift bins. The points are the measurements and the lines are a fit according to Eqs. 3.13 and 3.14 . 


\section{Halo-Cluster redshift relation}

In Fig. 6.18 the redshift of the matched pairs is shown, with the scatter and bias as a function of halo $z_{h}$ and cluster $z_{c l}$ redshift. The top panel represents the scatter and bias for the cluster redshift as a function of the true halo redshift $P\left(z_{c l} \mid z\right)$, and the right panel is the complementary distribution $P\left(z \mid z_{c l}\right)$. The scatter in both directions is almost a constant of $\approx 0.03$, much lower than the value of $\sigma_{z}$ used on the matching. Therefore, these scatter values were not artificially limited by the matching parameter $\sigma_{z}$, and should represent the values used to create the Gaussian photometric redshifts in the simulation.

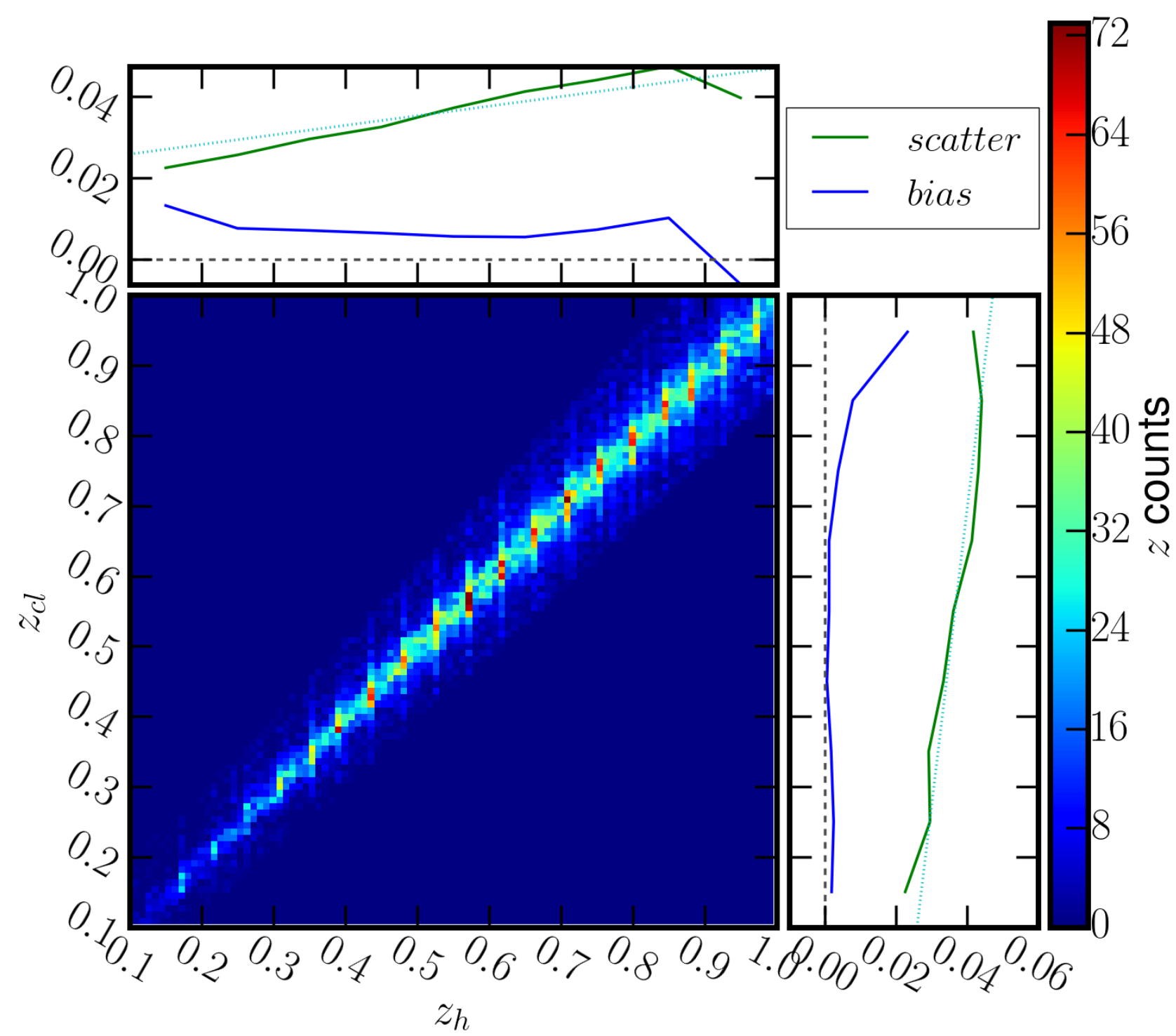

Figure 6.18: Relation between the halo and cluster redshifts in the BCC simulation.

As expected, the scatter is much smaller than the size of the redshift bins $(\Delta z=0.1)$ we 
use for the cluster abundance. Hence, the resulting effects on the cluster counts proved to be negligible. Although these redshift effects were described in the theoretical predictions, they will not be discussed in the further sections.

\section{Mass-Richness Relation}

As more massive dark matter halos should result in richer galaxy clusters, we expect the halo mass and corresponding cluster richness to be linked by a nearly linear scaling relation. It has been discussed in the literature that the mass-richness relation can be described by a functional form close to a power law (Koester et al., 2007). Thus the following parametrization was adopted here

$$
\log \left[\frac{M^{\mathrm{obs}}\left(N_{\mathrm{gal}}, z\right)}{M_{\odot} h^{-1}}\right]=A(z) \log \left[N_{\mathrm{gal}}\right]+B(z) \quad\left\{\begin{array}{l}
A(z)=A_{0}+A_{1}(1+z) \\
B(z)=B_{0}+B_{1}(1+z)
\end{array}\right.
$$

This relation will be used to convert mass to richness for a Gaussian mass-richness distribution $P\left(N_{\text {gal }} \mid M\right)$, according to the formalism developed in section 3.3.2.

$$
P\left(N_{\mathrm{gal}} \mid M\right)=\frac{1}{\sqrt{2 \pi \sigma_{\ln N_{\mathrm{gal}}}^{2}(M, z)}} \exp \left[-\frac{\left(\ln N_{\mathrm{gal}}-\ln N(M, z)\right)^{2}}{2 \sigma_{\ln N_{\mathrm{gal}}}^{2}(M, z)}\right]
$$

where $\ln N(M)$ is the inverse relation from Eq. 6.4. The first step to map out the distribution $P\left(M^{\text {obs }}\left(N_{\mathrm{gal}}\right) \mid M\right)$, which is also a function of redshift $z$, is to compute the mean value and scatter of the richness $N_{\text {gal }}$ for each value of halo mass and redshift. Ideally, we could fit a Gaussian for a given $(M, z)$ and compute the mean value and scatter from the first two moments of this Gaussian distribution. In practice, we can just bin the data in mass and redshift and measure the mean value and scatter from the data points in each bin (see section A.3). Then the mean value and scatter for each bin is used to fit parameters in Eq. 6.4 .

In the left panel of Fig. 6.19 we show the mass of each matched halo and the richness of the corresponding cluster (red dots) at different redshift slices, as well as the binned data with measured scatter (green points), and the fitted mean mass-richness relation (blue solid line). As there is a minimum value of the richness provided by the cluster finder, the low mass region of the mass-richness space could be missing missing a significant portion of low richness clusters. Hence, a mass cut was made in each redshift bin to avoid regions that could introduce a high bias 
on the mass-richness relation. Some bins were not used to fit the mass richness relation and are reported as yellow points with error bars. To evaluate how well the redshift dependency describe the data, we also fitted the mass-richness parameters $(A, B)$ in each redshift bin independently (cyan dashed line). The difference between these two fitting procedures can be explicitly seen in the right panel of Fig. 6.19, where the points are from individual fits in each redshift bin and the lines are global fits using all data. The resulting relation had coefficient of order of unity $A_{0}=1.52 \pm 0.23$, with a small dependency on redshift $A_{1}=-0.14 \pm 0.15$, and the linear coefficient $B_{0}=10.72 \pm 0.45$ with small redshift dependency $B_{1}=0.64 \pm 0.28$ as well. The most massive mass bins at $0.1 \leq z \leq 0.2$ (yellow bin) clearly has a different behavior from all other bins and has been excluded from the fits. This bin was composed of only three objects, being of small statistical contribution, and when considered, introduced a significant bias on all mass-richness parameters fitted. In the top right panel Fig. 6.19, the mass corresponding to different richnesses values are shown, with the values fitted individually in each redshift bin (points with error bars) and using all the data (solid lines). Even though the deviation of the individual fit of parameters in the first redshift bin was considerable, the corresponding mass was much closer to the fit using all data. In the resulting relation, a cluster of low richness $\left(N_{\text {gal }}=20\right)$ was related to a halo of mass $10^{13.1} \sim 10^{13.6} M_{\odot} h^{-1}$, a medium cluster $\left(N_{\text {gal }}=60\right)$ to $10^{13.9} \sim 10^{14.3} M_{\odot} h^{-1}$ and a high richness cluster $\left(N_{\text {gal }}=100\right)$ corresponded to halos of $10^{14.2} \sim 10^{14.5} M_{\odot} h^{-1}$.

The scatter of $P\left(M^{\mathrm{obs}}\left(N_{\mathrm{gal}}\right) \mid M\right)$ can also be measured from Fig. 6.19, and is represented by the error bars. As seen from this figure, and also from the results in section 5.1, the scatter decreases with halo mass. In order to capture this behavior, we propose the following functional form for the richness scatter

$$
\sigma_{\ln N_{\mathrm{gal}}}(M, z)=\frac{\sigma_{0}(z)}{1+\left(\frac{M}{M_{\sigma}(z)}\right)^{n_{\sigma}(z)}}\left\{\begin{array}{lll}
\sigma_{0}(z) & =\sigma_{00} & +\sigma_{01}(1+z) \\
\log M_{\sigma}(z) & =\log M_{\sigma 0} & +\log M_{\sigma 1}(1+z) \\
n_{\sigma}(z) & =n_{\sigma 0} & +n_{\sigma 1}(1+z)
\end{array}\right.
$$

In this parametrization, $\sigma_{0}$ is the plateau value at lower masses, $M_{\sigma}$ is a scale mass where the scatter halves its value and $n_{\sigma}$ controls how fast the decrease occurs.

The scatter parameters were fitted using the same approach for the mass-richness relation, i.e. using all the data and comparing with individual fits in each redshift bin, resulting 

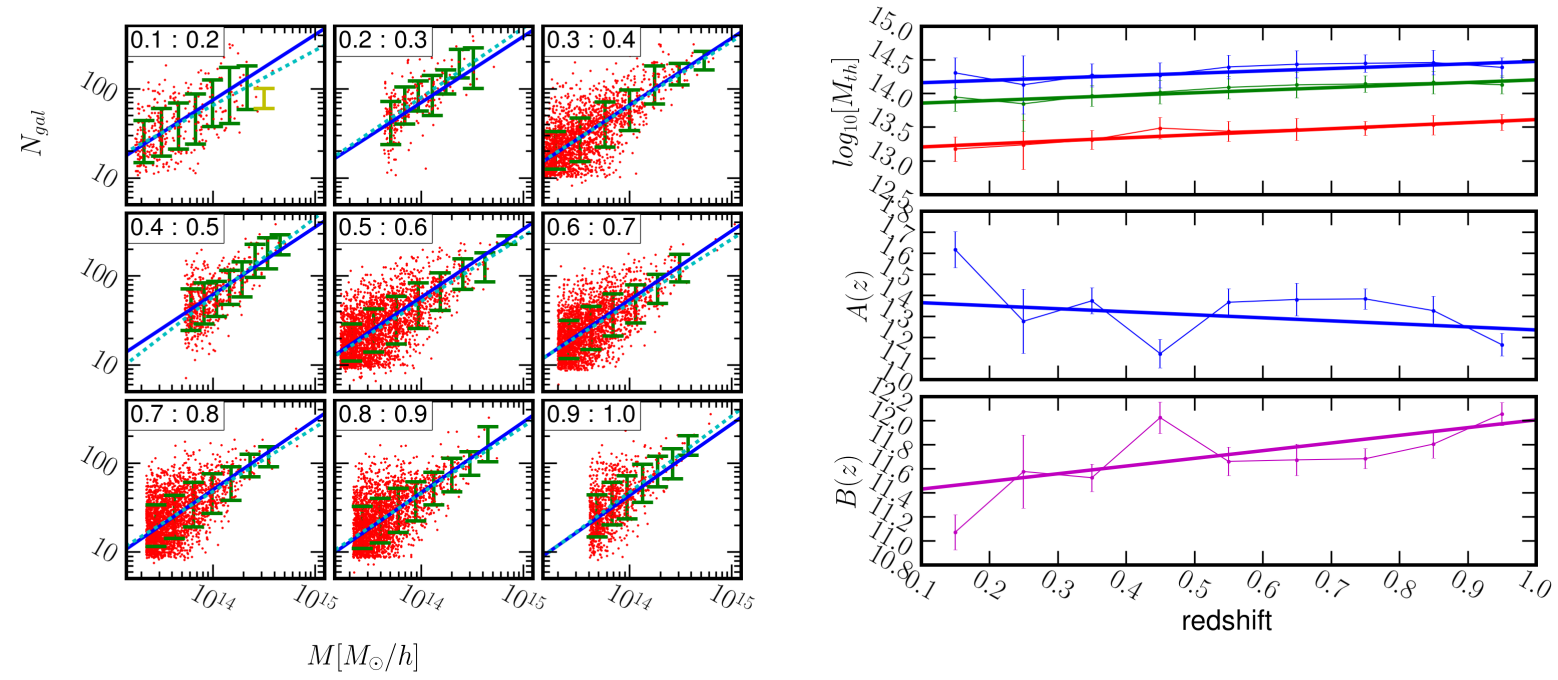

Figure 6.19: Mass Richness diagram for the matched halos with WaZp clusters in the BCC simulation and the fitted parameters. Left: The red dots are the matched pairs, the green points are the data binned with the measured scatter, the yellow points are the bins not used for the mass-richness fitting, the cyan dashed line is a fit for the mass-richness relation in each bin and the blue line is the redshift fit of the mass-richness parameters. Right: Parameters fitted in each redshift bin (points) and the redshift fit of the parameters (line). On Top all redshift bins were used for the fit and on the bottom the first bin was excluded, resulting in a better fit for the overall data.

in Fig. 6.20. In the left panel, the green points are the measured scatter in each mass bin, the yellow points are the measured scatter not used for the fits, the blue solid line is the fit with all the data and the cyan dashed lines were individually fitted in each redshift bin. In the right panel are the fitted parameters, where points are individual fits (corresponding to the cyan dashed lines in the left panel) and lines are the overall fit (corresponding to the blue solid lines in the left panel). The plateau parameter was $\sigma_{00}=0.208 \pm 0.047$, corresponding to a factor of $10^{\sigma_{00}} \approx 1.6$ in richness. The scale mass was in the same range of the mass of the halos considered $\log M_{\sigma_{0}}=14.99 \pm 0.18$, and the index was $n_{\sigma 0}=2.0 \pm 1.8$.

The first two redshift bins $(0.1 \leq z \leq 0.3)$ were excluded from the fit, as they presented very different behavior of the scatter compared to the other redshift bins. We should mention that these redshift bins had a much lower number of objects $\left(\mathcal{O}\left(10^{2}\right)\right.$ compared to the other bins $\left(\mathcal{O}\left(10^{3}\right)\right)$. The parameters presented a weak redshift dependence $\sigma_{01}=0.025 \pm 0.033$, $\log M_{\sigma_{1}}=-0.28 \pm 0.12, n_{\sigma 1}=-0.2 \pm 1.2$, all redshift compatible with null.

The highest mass bin at $0.8<z<0.9$ presented a high deviation from the expectation and, as it was composed of only a small number of objects (3 pairs), it was not used for the scatter fit. In fact, when it was considered, the plateau parameter $\sigma_{0}$ and the scale mass $M_{\sigma}$ were be 

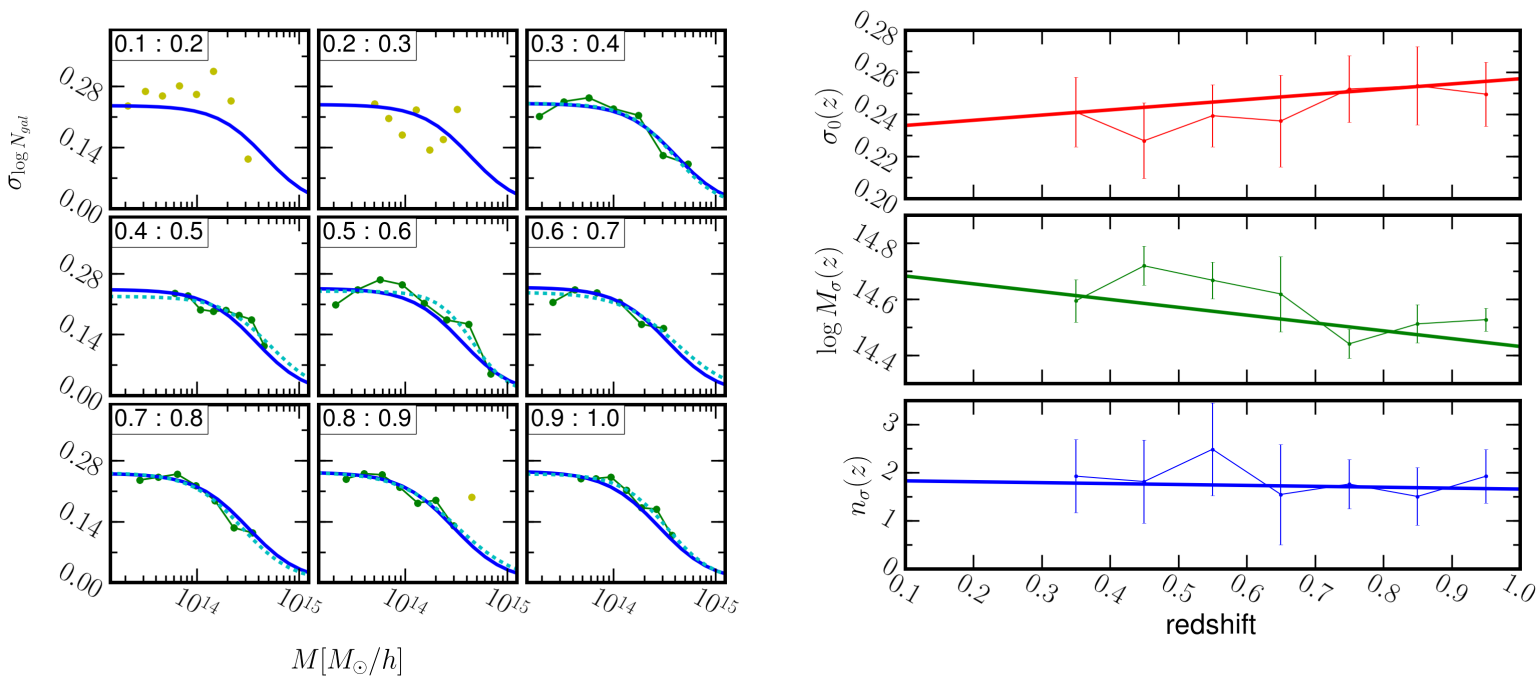

Figure 6.20: Measured scatter for each different binning. In the left the data was binned in mass and in the right in richness. The mass of the clusters (right plot) was computed from the fit. Each panel is the data in a redshift bin labeled at the bottom left.

very poorly constrained, having a small effect on the final fits for the scatter.

\section{Corrected Prediction for the Abundance of Clusters}

The final number counts of clusters was computed based on Eq. 3.20, using the functional forms fitted for the mass-richness relation, the scatter and also for the completeness and purity. To estimate the uncertainties on the abundance prediction, we propagated the variances of the fitted parameters $\Theta$. Based on the derivatives of the number counts with respect to each parameter $\Theta$ and the covariance matrix $C_{i j}(\Theta)$ of the parameters $\Theta$ measured in the fits, an estimated variance on the cluster counts can be computed as:

$$
\sigma_{\bar{m}}^{2}=\sum C_{i j}(\Theta) \frac{\partial \bar{m}}{\partial \Theta_{i}} \frac{\partial \bar{m}}{\partial \Theta_{j}}
$$

As the cluster abundance is extremely sensitive to the values of mass threshold, the mass richness parameters $\left(\Theta_{R M}\right)$ can be the main source of uncertainties. Hence only these parameters will be used to estimate the variance above. The partial derivatives of the number counts can be computed by computing the derivatives of $P\left(N_{\text {gal }} \mid M\right)$, the only term affected by these parameters: 

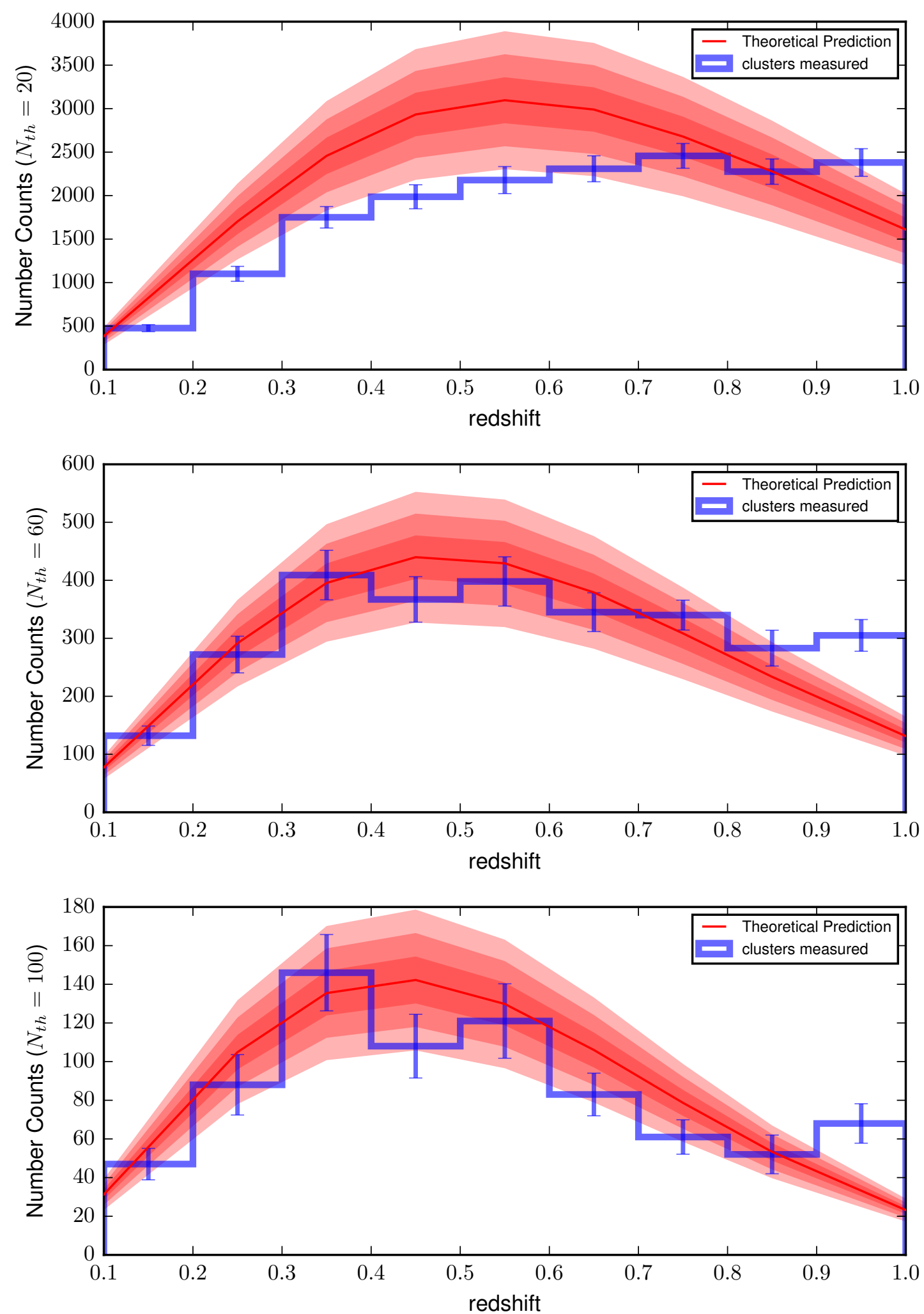

Figure 6.21: Number counts of WaZp clusters detected (blue steps) in a reduced region $(\approx 220$ $\mathrm{deg}^{2}$ ) of the BCC simulation and the corresponding theoretical prediction (red line) for three richness thresholds $N_{t h}=20,60,100$ (top to bottom). The shaded regions are the 1,2, $3 \sigma_{\bar{m}}$ computed with Eq. 6.7. 

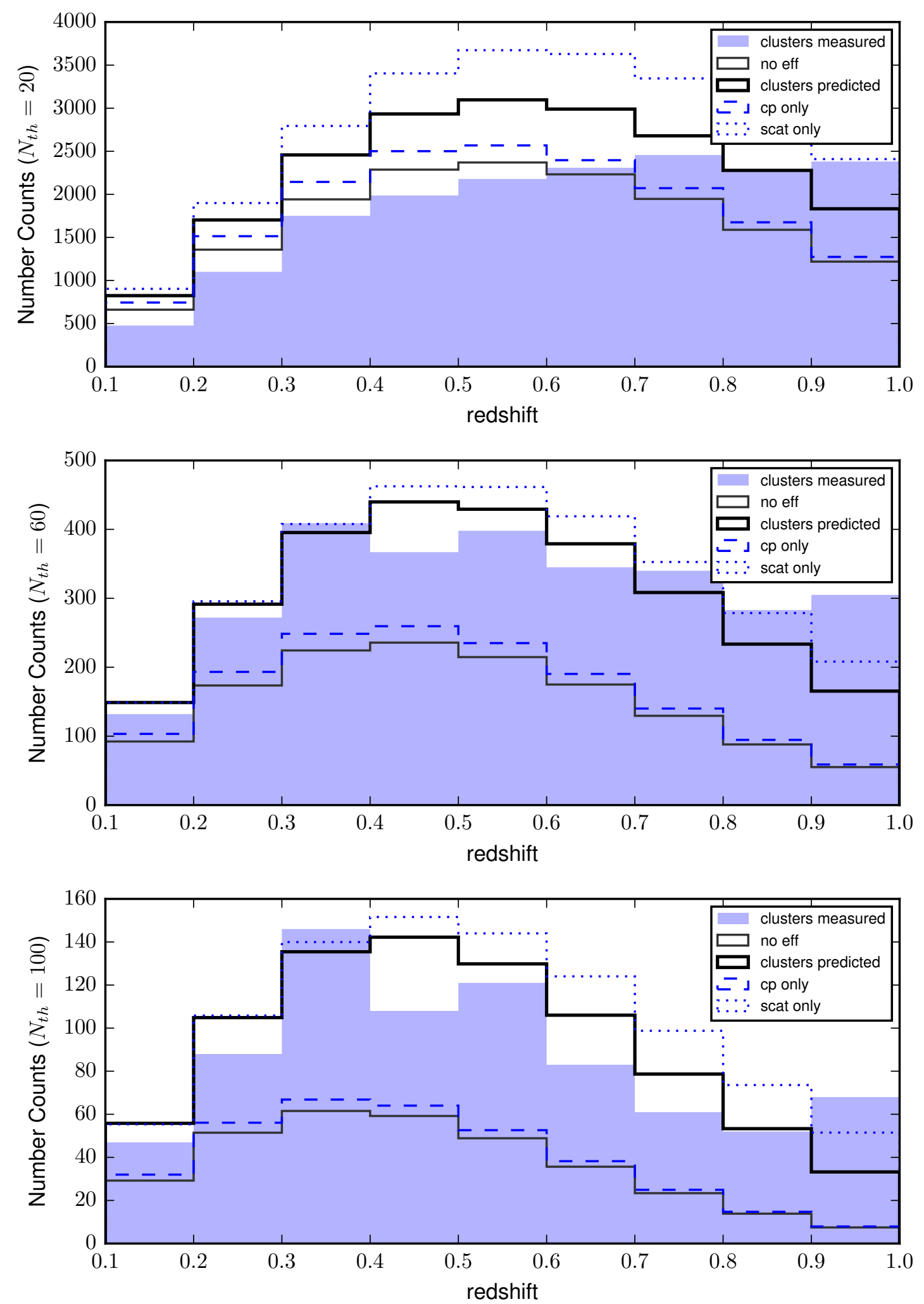

Figure 6.22: Number counts of WaZp clusters detected (blue filled regions) in the BCC simulation and theoretical prediction for three richness thresholds. Four approaches were considered: Perfect detection and no scatter on the mass-richness relation (thin black line); Including the completeness and purity, but no scatter (blue dashed line); Perfect detection with scatter (blue dotted line); Considering all observational effects (thick black line). 


$$
\begin{aligned}
\frac{\partial \bar{m}_{\alpha, i}}{\partial \Theta_{M R}} \equiv & \Delta \Omega \int_{z_{i}}^{z_{i+1}} d z \frac{D_{A}(z)^{2}}{H(z)} \int_{\ln N_{\mathrm{gal}}}^{\ln N_{\mathrm{gal}} \alpha+1} d \ln N_{\mathrm{gal}} \\
& \int d \ln M \frac{d \bar{n}}{d \ln M} \frac{c(M, z)}{p\left(N_{\mathrm{gal}}, z\right)} \frac{\partial P\left(N_{\mathrm{gal}} \mid M\right)}{\partial \Theta_{M R}} .
\end{aligned}
$$

Using a low $\left(N_{\mathrm{gal}}=20\right)$, intermediate $\left(N_{\mathrm{gal}}=60\right)$ and high $\left(N_{\mathrm{gal}}=100\right)$ richness thresholds, we estimated the expected abundance of clusters shown in Fig. 6.21. The blue steps are the measured cluster counts with total variance estimated using SSr (splitting the region in 87 parts), the red line is the theoretical prediction and the shaded regions are the 1,2 and $3 \sigma_{\bar{m}}$ levels computed from Eqs. 6.7 and 6.9. At the lower richness threshold, our prediction over-estimated the abundance of clusters for $z<0.7$. indicating the necessity of further investigating the modeling and measurements of the observational effects. However, for the other two richness thresholds, our prediction was in good agreement with the measurements up to $z \approx 0.8$. Hence, either the modeling or the measurements seem unreliable only at the lower richness clusters. At higher redshifts, the abundance of clusters is consistently larger than our predictions. These large counts at high redshifts do not follow the usual shape of cluster abundance, and there seems to be no indication of a different behavior at these redshifts in the measurements of the massrichness relation, the scatter, and the completeness and purity. This might be an issue of the WaZp cluster finder and we hope to investigate in the future.

In order to evaluate each observational effect individually, we also computed the theoretical prediction with different considerations in Fig. 6.22. First, we used the mass-richness relation measured, but disregarded the scatter, completeness and purity. Then we included one effect at a time, either the scatter or both completeness and purity. Finally, we composed the prediction with all these effects. As the scatter in the mass-richness distribution lowers the effective mass threshold (Lima \& Hu, 2007), the predictions without scatter should be below the abundance of clusters measured. It is the case for the intermediate and high richness thresholds, but not for the lower threshold. This appears to indicate a lower richness limit to the mass-richness relation fitted.

An additional interesting feature of the observational effects is that the cluster abundance increases when we include completeness and purity on the predictions without scatter (from thin black line to blue dashed line), but decreases if the scatter was already included (from blue dotted line to thick black line). To understand this effect, we computed the ratio of the 
completeness $c(M)$ over the purity $p\left(N_{\mathrm{gal}}\right)$ for a masses corresponding to the richness $\pm 1 \sigma_{\ln } N_{\mathrm{gal}}$, as shown in the left panel of Fig. 6.23. When we assume that there is no scatter, the distribution $P\left(N_{\text {gal }} \mid M\right)$ collapses into a delta Dirac and it is equivalent to simply assuming the mass of the clusters is perfectly computed from the mass-richness relation. In this case, the ratio $c / p$ is larger than 1 for $N_{\text {gal }} \gtrsim 10$ and the number counts increase. If the scatter is also considered, the ratio $c / p$ increases the influence of larger masses (green line) and reduces it for smaller masses. As the abundance of halos (and clusters) decreases with mass, the number of clusters drops. It is interesting to note that this figure shows that the completeness and purity measured have a behavior similar to that of case (1) from section 5.3 .
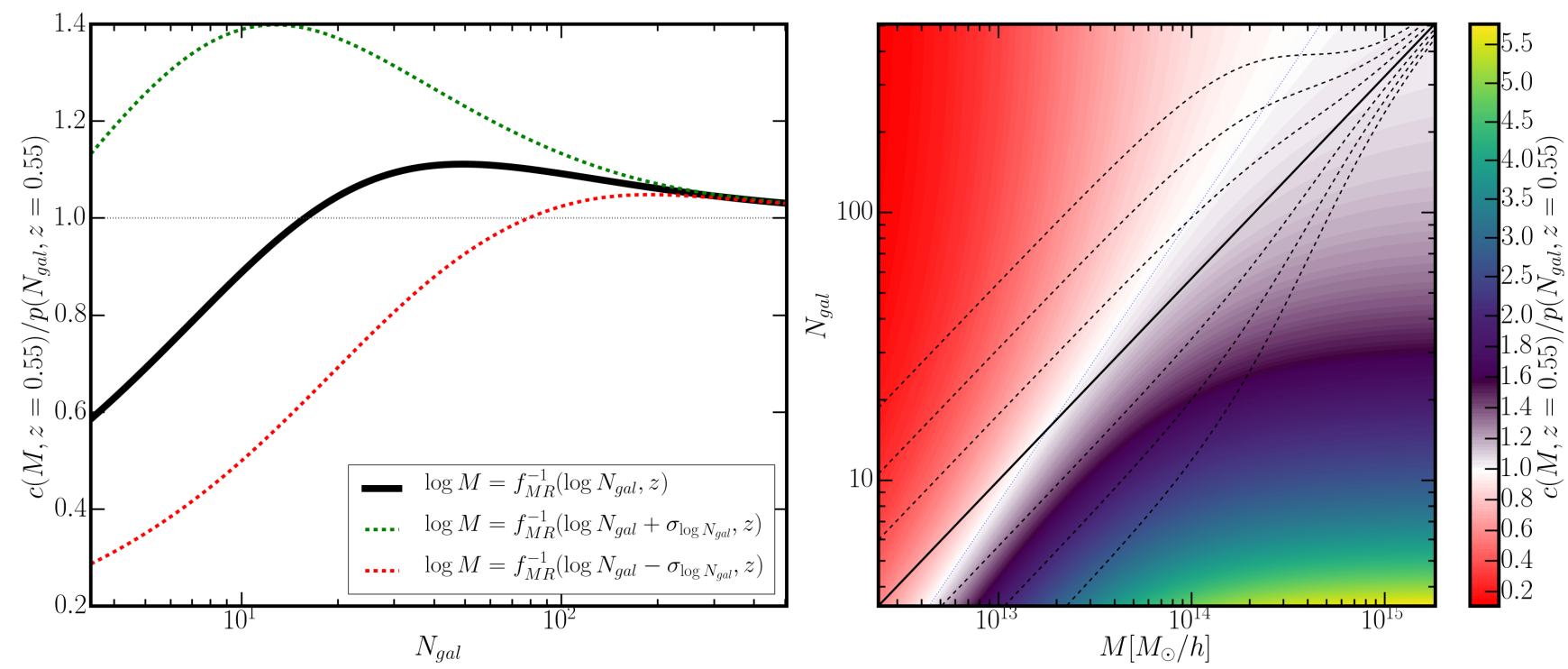

Figure 6.23: Ratio of completeness and purity for different masses/richnesses at $z=0.55$. Left: The black line corresponds to a mass computed form the richness using the mass richness relation, in the green line the mass was computed from a positive variation of $\sigma_{\ln N_{\text {gal }}}$ on the richness on the richness and on the red line with a negative. Right: The color map was arranged for red corresponding to $c<p$, white to $c=p$ and the other colors to $c>p$. The solid line is for the mass richness relation calibrated, and the dashed lines are for 1,2 and $3 \sigma$ variations.

In the right panel of Fig. 6.23, the ratio $c / p$ is shown as a color map as a function of mass and richness. The colors were arranged such that $c<p$ is represented by red, $c=p$ by white and $c>p$ by the other colors. The solid black line is the mass richness relation and the dashed lines are 1, 2 and $3 \sigma$ variations. Inside a $3 \sigma$ level, the local contribution of the $c / p$ can be from a factor of 0.2 up to 3.0. However, the real contribution is diluted by the integration over a certain volume in $\left(M, N_{\mathrm{gal}}\right)$ space. From the color scheme, it becomes clear that, in most cases, the ratio $c / p$ lessens the contribution of masses lower than the mass richness relation and increases the contribution of higher masses. 


\section{Chapter 7}

\section{Conclusions}

Galaxy clusters comprise a powerful tool for constraining cosmology. In combination with other probes, cluster properties have the potential to help distinguish between competing models of cosmic acceleration, including dark energy and modified gravity. However, neglecting the observational effects can lead to systematic biases on the cosmology recovered. The aim of this work has been to provide a framework for constraining cosmological parameters based on galaxy cluster abundance, including the main observational effects necessary for cluster cosmology. Whereas we have had some important preliminary results, a complete description of these issues is still a work in progress.

The main products from this work were divided into results from a theoretical standpoint and from simulations. In the theoretical results we studied the properties of the mass-observable distribution, the cosmological constraining power from dark matter halos, and the influence of optical effects on cluster dark energy constraints. In the simulation results, we analyzed the dark matter halo catalog from the MICE simulation to estimate halo covariances in multiple ways. We also used a simulation from DES collaboration to constrain cosmology and investigate observational effects of galaxy cluster detection.

In investigating the mass-observable distribution, we explored an example of $P\left(M \mid M^{\text {obs }}\right)$ derived from a given $P\left(M^{\mathrm{obs}} \mid M\right)$ with the Tinker mass function. The probability $P\left(M^{\mathrm{obs}} \mid M\right)$ was assumed to be Gaussian with fixed scatter and no bias. The resulting $P\left(M \mid M^{\text {obs }}\right)$ was also well approximated by a Gaussian in $M$, however, the mass scatter and bias presented a clear dependence with $M^{\text {obs }}$.

Using the Fisher Matrix (FM) formalism we estimated cosmological constraints from dark matter halos and also from galaxy clusters with conditions similar to the observations expected 
in current surveys. First we fixed the covariance matrix, focusing on the constraints from the abundance of clusters only. We analyzed the correlation between each pair of cosmological parameters, first fixing all other parameters and later marginalizing over the other parameters. When the parameters were fixed, we also studied the influence of the redshift range on the constraints. The correlation between parameters could be deduced from the manner in which each parameter affected the halo number counts. When a positive variation on both parameters increased or decreased the abundance of halos, the correlation is negative, and when a variation had opposite effects, the correlation is positive. A particular case occurs for $w$ variations, which decreases the halo number counts at lower redshifts $z \lesssim 1.0$ and increases it at higher redshifts. This causes the correlation of $w$ with the other parameters to have opposite values for low and high redshifts. Consequently, using a wide range of redshifts help providing tighter constraints on $w$.

When we tried to constrain all cosmological parameters $\left(\Omega_{m}, w, \sigma_{8}, h, n_{s}, \Omega_{b}\right)$ together with no priors, both the resulting constraining errors and the correlations among parameters were quite large. By applying some loose priors of $\sigma_{p}=1.0$ to all parameters in order to restrain the parameters to regions with physical meaning, the constraints and correlation were greatly improved. For some cosmological parameters $\left(h, n_{s}\right)$, the abundance of halos alone provided no significant information and had a final constraint of the order of the prior. Meanwhile for $w$ the constraint was of $\approx 3 \%$.

Using the covariance as well as the abundance of halos improved significantly the cosmological constraints. Without the need of physical priors, the constraints on $\left(\Omega_{m}, w, \sigma_{8}, h, n_{s}, \Omega_{b}\right)$ were of $(1.56 \%, 1.70 \%, 0.61 \%, 4.00 \%, 1.88 \%, 14.75 \%)$. This showed the importance of also using the covariance of the dark matter halos.

The effects of completeness and purity were investigated via the Fisher Matrix and the results were also present in (Aguena \& Lima, 2016). We considered a hypothetical survey with a threshold mass of $10^{13.8} M_{\odot} / h$, and with completeness and purity exerting a significant influence on masses lower than $\leq 10^{14.2} M_{\odot} h^{-1}$. There were two main behaviors for the ratio $c / p$ shown in table 5.1. For each case we found the lowest mass threshold in which it was possible to disregard completeness and purity effects and still have reliable dark energy constraints. Assuming perfect knowledge of the mass-richness relation and of completeness and purity parameters led to dark energy constraints of $\sigma\left(\Omega_{\mathrm{DE}}, w\right)=(0.006,0.033)$ for the case considered.

When we let both the richness-mass and completeness/purity parameters vary freely, the 
constraints suffer a degradation of $(70 \%, 36 \%)$. An important finding of our results is that, although there is significant degradation when all non-cosmological parameters are free - relative to the perfect knowledge case - the degradation relative to the case of fixed completeness and purity and free mass-richness parameters is very small $(4 \%, 2 \%)$. Therefore, if the massrichness parameters are kept free, including the completeness and purity parametrization does not deteriorate significantly the constraints on dark energy. We also showed that even though the normalized constraints of each mass-richness and completeness and purity parameter provided by the FM present a wide range of magnitudes $\left(10^{-1} \sim 10^{1} \%\right)$, priors on completeness and purity or on mass-richness parameters alone reach a threshold of improvement. The real gain would come from a combined prior of all nuisance parameters.

We investigated the influence of the limiting redshift and the total area focusing on the future surveys. If we expand the maximum redshift to 2.0 , there is an improvement of $(22 \%, 28 \%)$ on $\sigma\left(\Omega_{\mathrm{DE}}, w\right)$ for case (1). The degradation caused by completeness and purity effects is computed using the ratio between the constraint with all free parameters $\sigma\left[\Theta_{D E} \mid \sigma_{p}\left(\Theta_{C P}\right)=\infty\right]$ and fixing $c / p$ parameters $\sigma\left[\Theta_{D E} \mid \sigma_{p}\left(\Theta_{C P}\right)=0\right]$. The ratio $\sigma\left[\Theta_{D E} \mid \sigma_{p}\left(\Theta_{C P}\right)=\infty\right] / \sigma\left[\Theta_{D E} \mid \sigma_{p}\left(\Theta_{C P}\right)=0\right]$ presents a minimum at $z_{\max } \approx 1$, and increases as we consider higher $z_{\max }$. However, this does not mean the constraint on the dark energy parameters degrades when we include higher redshifts. What happens is much more information is added with the higher redshifts and $\sigma\left[\Theta_{D E} \mid \sigma_{p}\left(\Theta_{C P}\right)=0\right]$ improves remarkably, while $\sigma\left[\Theta_{D E} \mid \sigma_{p}\left(\Theta_{C P}\right)=\infty\right]$ reaches a minimum plateau. The constraints improve with the square root of the total survey area because we divide the survey in sub-samples of the same fixed area, here set to $10 \mathrm{deg}^{2}$. Therefore, by doubling our area $(1 / 4$ th of sky) there is an improvement of $\approx 29 \%$ and in the full-sky limit the constraints improve by $\approx 65 \%$.

In the covariance estimation of the MICE dark matter halo simulation, we divided the catalog into equal pieces with several approaches to the number of divisions and different covariance estimators. Using Sub-sampling rescaled, Jack-Knife and Bootstrap produced essentially the same results, recovering the covariance of the full angular area with a precision of $10-15 \%$. We also compared these measurements with our theoretical predictions using the Tinker mass function and bias, and found a considerable deviation. However, Hoffmann et al. (2015) showed that the Tinker mass function is not the best fit to the MICE simulation (Fosalba et al., 2015b; Crocce et al., 2015; Fosalba et al., 2015a) and required recalibration. Hence, would expect a better agreement with the data if the bias were also a re-calibrated in the simulation. 
We analyzed the halo catalog from the Aardvark v1.0 catalog of the BCC simulations from the DES. To optimize the cosmological constraints, we determined the largest area with a robust covariance measurement. The constraints were made using MCMC, but Fisher Matrix forecasts were also projected, corroborating the results. When only some cosmological parameters $\left(\Omega_{m}, w, \sigma_{8}\right)$ were considered, the constraints were of $(7 \%, 9 \%, 1 \%)$ and agreed with FM results. When all parameters were allowed to vary, the constraints on $\left(\Omega_{m}, w, \sigma_{8}\right)$ only suffered a small degradation, and $n_{s}$ could be constrained at a $10 \%$ level. However, the constraints on parameters $\left(h, \Omega_{b}\right)$ did not converge to the expected values, indicating a need for external priors.

We also considered a simulated cluster catalog, which was obtained from applying the WaZp cluster finder on the BCC simulation. This analysis provided lots of information for the treatment of observational effects. A proposed functional form for completeness/purity seemed to be able to fit the simulated data successfully, serving therefore as a reasonable functional form for use on real data. The functional forms proposed for the mean value of the mass-richness relation and the scatter also proved to fit well the measured simulated data. As predicted, the scatter in the $\left(z_{c l}, z_{h}\right)$ relation was very small and did not have any substantial effect on the cluster abundance prediction. The final abundance of clusters modeled with the proposed functional forms and fitted parameters agreed reasonably with the abundance of detected clusters, especially those of high richness. Hence, this framework seems to be robust for use in order to obtain cosmological constraints with real observations.

Throughout the development of the work described in this thesis, several numerical codes have been developed from scratch and constantly improved. These codes provide a foundational pipeline that can be used for multiple aspects of cluster analysis, which include comparing cluster catalogs and obtaining reliable cosmological constraints from large photometric surveys such as the DES. We are currently using these tools to analyze a WaZp catalog based on the first year data release of the DES, and we hope to submit these results to publication soon. Likewise, we expect to apply these methods to future DES data releases as well as to nextgeneration surveys. Additionally, the matching pipeline is being integrated in the DES Science portal at LIneA, widening its applications and facilitating collaboration with other groups and the combination of different cosmological probes. 


\section{Appendices}





\section{Appendix A}

\section{Estimating Galaxy Cluster Properties in}

\section{Simulations}

In simulations, where the dark matter halos are perfectly known, an analysis of their relation to the galaxy clusters (in terms of completeness and purity) can be conducted. The main disparity between the abundance of halo and cluster observed, however, will be originated from the particular cluster finder used. Different cluster finders which work properly should find similar distributions of detected clusters with similar properties. Thus, simulations provide a great opportunity to study the characteristics and performances of multiple cluster finders.

\section{A.1 Matching of Halos and Clusters}

Once a cluster finder is run on a simulation, the clusters detected can be compared with the dark matter halos by a matching process. The two main approaches used are the proximity matching - where the distance between the objects is used - and the membership matching - where the member galaxies of clusters and halos are compared. Both approaches have an intrinsic parameter for considering the matching successful. For the proximity matching it is the maximum distance between the center of the halo and cluster, and for the membership matching it is the minimum fraction of objects the halo-cluster pair has in common.

There are two distinct considerations we must make during the matching process. First, as described above, it must be defined what conditions a halo-cluster pair must obey to be considered a possible match (what is the maximum distance or the minimum fraction of shared members). The second step is to establish which halos/clusters will be prioritized in the case 
132 APPENDIX A. ESTIMATING GALAXY CLUSTER PROPERTIES IN SIMULATIONS

\begin{tabular}{c|c|l}
\hline \hline Case & Name & Description \\
\hline \hline 1 & Halo Multiple Match & $\begin{array}{l}\text { Matching clusters to halos, allowing the same cluster to } \\
\text { match multiple halos. }\end{array}$ \\
\hline 2 & Cluster Multiple Match & $\begin{array}{l}\text { Matching halos to clusters, allowing the same halo to match } \\
\text { multiple cluster. }\end{array}$ \\
\hline 3 & Multiple Match & $\begin{array}{l}\text { Consider a halo/cluster to have a match if it is matched in } \\
\text { either case 1 and/or 2. }\end{array}$ \\
\hline 5 & Halo Exclusive Match & $\begin{array}{l}\text { Matching clusters to halos, requiring each cluster to match } \\
\text { only one halo. }\end{array}$ \\
\hline 6 & Cluster Exclusive Match & $\begin{array}{l}\text { Matching halos to clusters, requiring each halo to match } \\
\text { only one cluster. }\end{array}$ \\
\hline \hline
\end{tabular}

Table A.1: Different types of matching criteria of interest between dark matter halos and galaxy clusters.

of multiple matches, i.e. when it is possible to find more than one pair that satisfies the first matching condition for a given halo/cluster.

One approach for selecting the best candidate for multiple matches is to prioritize the mass of the halos and the mass-proxy of the cluster (such as the richness). As the most massive halos should form richer clusters that are easier to detect, one can expect more accurate results for this group. Therefore it is reasonable to consider e.g. that more massive halos should be prioritized on the matching process to richer clusters. In this process, halos are sorted by mass and clusters by richness, assuring more massive halos to find richer clusters.

Another approach for solving multiple matches could be simply choosing the nearest neighbor, i.e. selecting the closest candidate in proximity matching, or the pair with a higher percentage of shared object in membership matching.

Once these conditions have been defined, there are several possible outcomes from the halocluster matching according to the possibility of multiple matches, whether we match clusters to halos or halos to clusters, and refinements with cross match. The main cases of interest are displayed in Table A.1.

In case 1 , we try to match a cluster to each halo, allowing for the same cluster to be matched to several halos, while in case 2 we make the same process exchanging halos with clusters. In these two cases, each halo can be associated with multiple clusters and each cluster can be associated to multiple halos. Among other reasons, this may happen when the cluster finder breaks a halo into artificial substructures or merges two halos into a single cluster. Notice that 
cases 1 and 2 are indeed different, as a matching condition may be met by a halo but not by the respective cluster. For instance, if the matching condition is based on angular proximity and related to the objects sizes, a large halo can match a small cluster, but the inverse might not be true. The same may occur in membership matching, where the fraction of common members between a halo and a cluster differ if they do not have the same total number of members.

For cases with multiple matches $(1,2,3)$, we only have to check if there is a candidate satisfying the matching condition, and the resulting completeness and purity are larger than for cases with exclusive matches $(4,5,6)$. However, since there is not a one-to-one correspondence between clusters and halos in multiple matches cases, it is not possible to make comparisons between properties of halos and clusters such as the mass-proxy relation. These cases should be used as a first quality test of the cluster finder, to inspect clusters that are being found in regions with halos. Again, notice that cases $(1 \& 2)$ give us a list of matched halos and clusters, respectively, and the results of each case can be different. Case 3 corresponds to a somewhat more robust approach, where we consider a halo/cluster to have a match if it is matched in either case 1 or 2 .

In cases 4 and 5, it is required the matches to be exclusive, i.e. each cluster must be matched to only one halo and vice-versa. This assures a one-to-one correspondence between halos and clusters, allowing us to make direct comparisons between the properties of halos and clusters, number of member galaxies, etc. Additionally, it is possible to derive a relation between the mass of dark matter halos and the equivalent proxy of the galaxy cluster. However, the question of choosing between case 4 and 5 still remains, as both matching approaches can produce different results. It has been argued by Rozo \& Rykoff (2014) that for cosmological purposes, a two-way match should be used, resulting in case 6 . In this case, only the same pairs found by in cases 4 and 5 are considered. This process should provide more reliable halo-cluster pairs, whose matching take into account the properties of both halos and clusters.

\section{A.2 Measuring Completeness and Purity}

Completeness and purity not only are quantities that help evaluate the performance of a cluster finder in a given survey, but that must also be taken into account in the cluster abundance prediction (Aguena \& Lima, 2016). However, only for simulations it is possible to compute properly the completeness and purity of a cluster finder. Once the dark matter halos and galaxy 
clusters have been matched, measuring these selection functions is simply a matter of comparing the number of matched objects to the total number of halos and clusters. The completeness can be defined as the ratio between the number of pairs matched and the number of halos, as a complete catalog has a cluster for each halo. Because the completeness is related to halos, it can be measured as a function of the true halo redshift $z$ and mass $M$

$$
C(z, M)=\frac{N_{\text {match }}(z, M)}{N_{\text {halo }}(z, M)}
$$

The purity is defined as the ratio between the number of matched pairs and the total number of clusters. As the purity describes properties of the clusters, it is measured in terms of the observed redshift $z^{o b s}$ (that can be photometric), and the observable mass of the cluster $M^{\text {obs }}$ (or equivalently its proxy $Y$ )

$$
P\left(z^{o b s}, M^{\text {obs }}\right)=\frac{N_{\text {match }}\left(z^{o b s}, M^{\text {obs }}\right)}{N_{\text {cluster }}\left(z^{o b s}, M^{\text {obs }}\right)}=\frac{N_{\text {match }}\left(z^{o b s}, Y\right)}{N_{\text {cluster }}\left(z^{o b s}, Y\right)}
$$

\section{A.3 Estimating the Mass-proxy relation}

The galaxy cluster abundance is very much dependent on the effective mass threshold above which we count objects. Thus detailing the mass-proxy distribution is of up-most importance. As described in section 3.3.2, the relation between mass $M$ and proxy $Y$ can be characterized by a probability distribution $P(Y \mid M)$. In fact, accurate predictions of cluster counts require mapping out the whole distribution between the mass and the proxy, not just the mean relation.

From a practical stand point, analyzing $P(Y \mid M)$ means fixing the mass and investigating the distribution of the proxy. When working with simulations of optical data, this can be done by binning the matched pairs as a function of the halo masses and evaluating the richness distribution in each bin. Then given a functional form of $P(Y \mid M)$, we fit its parameters in each mass bin. If $P(Y \mid M)$ is modeled as a Gaussian (such as in Eq. 3.3.2), there are two components that define it: the mean mass-proxy relation $\langle\ln Y \mid \ln M\rangle$ and the scatter around this mean relation $\sigma_{\ln Y}$. Once functional forms for these two features are proposed (e.g. as a function of mass and redshift), the parameters can be measured directly. As it is known for points generated by a Normal distribution, the expectation value and variance $\left(\langle\ln Y \mid \ln M\rangle\right.$ and $\sigma_{\ln Y}^{2}$ in this case $)$ can be recovered by measuring the mean value and scatter of the points. 


\section{Bibliography}

Abdalla F. B., Banerji M., Lahav O., Rashkov V., 2011, Mon. Not. R. Astron. Soc. , 417, 1891

Abramo L. R., et al., 2012, Mon. Not. R. Astron. Soc. 423, 3251

Aguena M., Lima M., 2016, preprint, (arXiv:1611.05468)

Allen S. W., Evrard A. E., Mantz A. B., 2011, Annual Review of Astronomy and Astrophysics, 49,409

Amara A., Réfrégier A., 2008, Mon. Not. R. Astron. Soc., 391, 228

Amendola L., et al., 2013, Living Reviews in Relativity, 16

Arnouts S., Ilbert O., 2011, LePHARE: Photometric Analysis for Redshift Estimate, Astrophysics Source Code Library (ascl:1108.009)

Arnouts S., et al., 2007, Astronomy and Astrophysics, 476, 137

Bardeen J. M., Bond J. R., Kaiser N., Szalay A. S., 1986, Astrophysical Journal, 304, 15

Bartelmann M., Schneider P., 2001, Phys. Rep., 340, 291

Baxter E. J., Rozo E., Jain B., Rykoff E., Wechsler R. H., 2016, Mon. Not. R. Astron. Soc., 463,205

Behroozi P., Wechsler R., Wu H.-Y., 2012, Rockstar: Phase-space halo finder, Astrophysics Source Code Library (ascl:1210.008)

Behroozi P. S., Wechsler R. H., Wu H.-Y., 2013, Astrophysical Journal, 762, 109

Bell E. F., et al., 2004, Astrophysical Journal, 608, 752

Benítez N., 2000, Astrophysical Journal, 536, 571 
Bocquet S., Saro A., Dolag K., Mohr J. J., 2016, Mon. Not. R. Astron. Soc., 456, 2361

Botzler C. S., Snigula J., Bender R., Hopp U., 2004, Mon. Not. R. Astron. Soc. , 349, 425

Boylan-Kolchin M., Springel V., White S. D. M., Jenkins A., Lemson G., 2009, Mon. Not. R. Astron. Soc. , 398, 1150

Camacho H., Lima M., Sobreira F., de Simoni F., 2017, In preparation

Carlstrom J. E., Holder G. P., Reese E. D., 2002, Annual Review of Astronomy and Astrophysics, 40, 643

Carrasco Kind M., Brunner R. J., 2013, Mon. Not. R. Astron. Soc., 432, 1483

Carrasco Kind M., Brunner R., 2014, MLZ: Machine Learning for photo-Z, Astrophysics Source Code Library (ascl:1403.003)

Carroll S. M., Press W. H., Turner E. L., 1992, Annual Review of Astronomy and Astrophysics, 30,499

Castignani G., Benoist C., 2016, A\&A, 595, A111

Catelan P., Lucchin F., Matarrese S., Porciani C., 1998, Mon. Not. R. Astron. Soc. , 297, 692

Cenarro A. J., et al., 2012, in Observatory Operations: Strategies, Processes, and Systems IV. p. 84481A, doi:10.1117/12.925432

Christensen N., Meyer R., Knox L., Luey B., 2001, Classical and Quantum Gravity, 18, 2677

Clerc N., et al., 2016, Mon. Not. R. Astron. Soc., 463, 4490

Cole S., Kaiser N., 1989, Mon. Not. R. Astron. Soc. , 237, 1127

Crocce M., Castander F. J., Gaztañaga E., Fosalba P., Carretero J., 2015, Mon. Not. R. Astron. Soc. 453,1513

Cui W., Borgani S., Dolag K., Murante G., Tornatore L., 2012, Mon. Not. R. Astron. Soc. , 423, 2279

Dietrich J. P., Hartlap J., 2010, Mon. Not. R. Astron. Soc., 402, 1049

Dietrich J. P., et al., 2014, Mon. Not. R. Astron. Soc. 443, 1713 
Dunkley J., Bucher M., Ferreira P. G., Moodley K., Skordis C., 2005, Mon. Not. R. Astron. Soc. 356,925

Efron B., 1992, Bootstrap methods: another look at the jackknife. Springer New York

Eke V. R., Cole S., Frenk C. S., 1996, Mon. Not. R. Astron. Soc. , 282, 263

Erickson B. M. S., Cunha C. E., Evrard A. E., 2011, Physical Review D, 84, 103506

Evrard A. E., Metzler C. A., Navarro J. F., 1996, Astrophysical Journal, 469, 494

Evrard A. E., et al., 2002, Astrophysical Journal, 573, 7

Farahi A., Evrard A. E., Rozo E., Rykoff E. S., Wechsler R. H., 2016, Mon. Not. R. Astron. Soc. 460,3900

Farrens S., Abdalla F. B., Cypriano E. S., Sabiu C., Blake C., 2011, Mon. Not. R. Astron. Soc. П. 417, 1402

Few C. G., Courty S., Gibson B. K., Kawata D., Calura F., Teyssier R., 2012, Mon. Not. R. Astron. Soc., 424, L11

Flaugher B., 2005, International Journal of Modern Physics A, 20, 3121

Flaugher B., et al., 2015, Astron. J., 150, 150

Foreman-Mackey D., Hogg D. W., Lang D., Goodman J., 2013, PASP, 125, 306

Fosalba P., Gaztañaga E., Castander F. J., Crocce M., 2015a, Mon. Not. R. Astron. Soc., 447, 1319

Fosalba P., Crocce M., Gaztañaga E., Castander F. J., 2015b, Mon. Not. R. Astron. Soc. 448, 2987

Frieden B. R., 2004, Science from Fisher Information: A Unification, 2 edn. Cambridge University Press, http://www. worldcat.org/isbn/0521810795

Gladders M. D., Yee H. K. C., 2005, Astrophysical Journal Supplement, 157, 1

Gladders M. D., Yee H. K. C., Majumdar S., Barrientos L. F., Hoekstra H., Hall P. B., Infante L., 2007, Astrophysical Journal, 655, 128 
Gorski K., Hivon E., Banday A., Wandelt B., Hansen F., et al., 2005, Astrophys.J., 622, 759

Hamilton A. J. S., 2000, Mon. Not. R. Astron. Soc., 312, 257

Hinshaw G., et al., 2013, Astrophysical Journal Supplement, 208, 19

Hoffmann K., Bel J., Gaztañaga E., 2015, Mon. Not. R. Astron. Soc., 450, 1674

Holder G., Haiman Z., Mohr J. J., 2001, Astrophysical Journal Letters, 560, L111

Hu W., Cohn J. D., 2006, Physical Review D, 73, 067301

Hu W., Kravtsov A. V., 2003, Astrophysical Journal, 584, 702

Huchra J. P., Geller M. J., 1982, Astrophysical Journal, 257, 423

Ilbert O., et al., 2006, Astronomy and Astrophysics, 457, 841

Ilbert O., et al., 2009, Astrophysical Journal, 690, 1236

Jenkins A., Frenk C. S., White S. D. M., Colberg J. M., Cole S., Evrard A. E., Couchman

H. M. P., Yoshida N., 2001, Mon. Not. R. Astron. Soc., 321, 372

Kacprzak T., et al., 2016, Mon. Not. R. Astron. Soc., 463, 3653

Kaiser N., 1984, Astrophysical Journal Letters, 284, L9

Koester B. P., et al., 2007, Astrophysical Journal, 660, 221

Komatsu E., et al., 2011, Astrophysical Journal Supplement, 192, 18

Kowalski M., et al., 2008, Astrophysical Journal, 686, 749

Kravtsov A. V., Vikhlinin A., Nagai D., 2006, Astrophysical Journal, 650, 128

Lacasa F., Lima M., Aguena M., 2016, preprint, (arXiv:1612.05958)

Lacey C., Cole S., 1994, Mon. Not. R. Astron. Soc., 271, 676

Levine E. S., Schulz A. E., White M., 2002, Astrophysical Journal, 577, 569

Lewis A., Bridle S., 2002, Physical Review D, 66, 103511

Lewis A., Challinor A., Lasenby A., 2000, Astrophys. J., 538, 473 
Lima M., Hu W., 2004, Physical Review D, 70, 043504

Lima M., Hu W., 2005, Physical Review D, 72, 043006

Lima M., Hu W., 2007, Physical Review D, 76, 123013

Ma C.-P., Bertschinger E., 1995, Astrophysical Journal, 455, 7

Ma Z., Hu W., Huterer D., 2006, Astrophysical Journal, 636, 21

Majumdar S., Mohr J. J., 2004, Astrophysical Journal, 613, 41

Marian L., Smith R. E., Hilbert S., Schneider P., 2012, Mon. Not. R. Astron. Soc., 423, 1711

Mazure A., et al., 2007, Astronomy and Astrophysics, 467, 49

Metropolis N., Rosenbluth A. W., Rosenbluth M. N., Teller A. H., Teller E., 1953, Journal of Chemical Physics, 21, 1087

Miller C. J., et al., 2005, Astron. J., 130, 968

Mo H. J., White S. D. M., 1996, Mon. Not. R. Astron. Soc., 282, 347

Mohr J. J., et al., 2012, in Society of Photo-Optical Instrumentation Engineers (SPIE) Conference Series. p. 0 (arXiv: 1207.3189), doi:10.1117/12.926785

Nagai D., 2006, Astrophysical Journal, 650, 538

Oyaizu H., Lima M., Cunha C. E., Lin H., Frieman J., Sheldon E. S., 2008, Astrophysical Journal, 674, 768

Peacock J. A., 1999, Cosmological Physics

Peebles P. J. E., 1980, The large-scale structure of the universe

Peel A., Lin C.-A., Lanusse F., Leonard A., Starck J.-L., Kilbinger M., 2017, Astronomy and Astrophysics, 599, A79

Perlmutter S., et al., 1997, Astrophysical Journal, 483, 565

Pierre M., et al., 2004, Journal of Cosmology and Astroparticle Physics, 9, 011

Planck Collaboration et al., 2016, Astronomy and Astrophysics, 594, A13 
Planelles S., Quilis V., 2010, Astronomy and Astrophysics, 519, A94

Pratt J. W., 1976, The Annals of Statistics, 4, 501

Predehl P., et al., 2010, in Space Telescopes and Instrumentation 2010: Ultraviolet to Gamma Ray. p. 77320U (arXiv:1001.2502), doi:10.1117/12.856577

Press W. H., Schechter P., 1974, Astrophysical Journal, 187, 425

Quenouille M. H., 1949, Ann. Math. Statist., 20, 355

Riess A. G., et al., 1998, Astrophysical Journal, 116, 1009

Rozo E., Rykoff E. S., 2014, Astrophysical Journal, 783, 80

Rozo E., et al., 2007, ArXiv Astrophysics e-prints, arXiv:astro-ph/0703571

Rozo E., Rykoff E. S., Bartlett J. G., Melin J.-B., 2015a, Mon. Not. R. Astron. Soc., 450, 592

Rozo E., Rykoff E. S., Becker M., Reddick R. M., Wechsler R. H., 2015b, Mon. Not. R. Astron. Soc. 453,38

Rykoff E. S., et al., 2014, Astrophysical Journal, 785, 104

Sánchez C., et al., 2014, Mon. Not. R. Astron. Soc., 445, 1482

Sarazin C. L., 1986, Reviews of Modern Physics, 58, 1

Saro A., et al., 2015, Mon. Not. R. Astron. Soc. , 454, 2305

Schneider P., Ehlers J., Falco E. E., 1992, Gravitational Lenses

Seljak U., Zaldarriaga M., 1996, Astrophysical Journal, 469, 437

Sheth R. K., Tormen G., 1999, Mon. Not. R. Astron. Soc., 308, 119

Sheth R. K., Mo H. J., Tormen G., 2001, Mon. Not. R. Astron. Soc. , 323, 1

Simet M., McClintock T., Mandelbaum R., Rozo E., Rykoff E., Sheldon E., Wechsler R. H., 2017, Mon. Not. R. Astron. Soc., 466, 3103

Soares-Santos M., et al., 2011, Astrophysical Journal, 727, 45 
Sobreira F., de Simoni F., Rosenfeld R., da Costa L. A. N., Maia M. A. G., Makler M., 2011, Physical Review D, 84, 103001

Springel V., 2005, Mon. Not. R. Astron. Soc., 364, 1105

Sunyaev R. A., Zeldovich Y. B., 1970, Astrophysics and Space Science, 7, 3

Tang J.-Y., Weller J., Zablocki A., 2006, ArXiv Astrophysics e-prints, astro-ph/0609028

Tegmark M., 1997, Physical Review Letters, 79, 3806

Tegmark M., Taylor A. N., Heavens A. F., 1997, Astrophysical Journal, 480, 22

Tinker J., Kravtsov A. V., Klypin A., Abazajian K., Warren M., Yepes G., Gottlöber S., Holz D. E., 2008, Astrophysical Journal, 688, 709

Tinker J. L., Robertson B. E., Kravtsov A. V., Klypin A., Warren M. S., Yepes G., Gottlöber S., 2010, Astrophysical Journal, 724, 878

Tyson J. A., 2002, in Tyson J. A., Wolff S., eds, Observatory Operations: Strategies, Processes, and Systems IVVol. 4836, Survey and Other Telescope Technologies and Discoveries. pp 10-20 (arXiv: astro-ph/0302102), doi:10.1117/12.456772

Vikhlinin A., Kravtsov A., Forman W., Jones C., Markevitch M., Murray S. S., Van Speybroeck L., 2006, Astrophysical Journal, 640, 691

Wang S., Haiman Z., Hu W., Khoury J., May M., 2005, Physical Review Letters, 95, 0011302

York D. G., et al., 2000, Astron. J., 120, 1579

Zaldarriaga M., Seljak U., 2000, The Astrophysical Journal Supplement Series, 129, 431

Zaldarriaga M., Seljak U., Bertschinger E., 1998, Astrophysical Journal, 494, 491

Zel'dovich Y. B., 1970, Astronomy and Astrophysics, 5, 84

de Simoni F., et al., 2013, Mon. Not. R. Astron. Soc., 435, 3017 